\title{
Experimental Modal Analysis of Business Jet Fuselage Tail Section Sub-Assemblies
}

by

Ian Donaldson

A thesis submitted to the Department of Mechanical and Materials Engineering

In conformity with the requirements for

the degree of Master of Applied Science

\author{
Queen's University \\ Kingston, Ontario, Canada
}

(October, 2020)

Copyright (C) Ian Donaldson, 2020 


\begin{abstract}
Experimental modal testing is a technique through which the dynamic response characteristics of a system can be found. Parameters such as the natural frequencies and mode shapes of a system can be extracted through experimentation, and these results can be used to confirm computational models, and/or provide insights from which improvements can be made to improve dynamic response characteristics. This thesis provides an overview of experimental modal analysis performed on two half scale aircraft fuselage subassemblies using shaker excitation. Prior to experimentation, relevant literature was reviewed. This provided the necessary foundation of theory and prior experiments which helped to form the experimental methodology presented. This methodology including the construction of each structure, data acquisition parameters, and validity checks, is covered in detail. Linearity and repeatability checks were used to validate the testing methodology in accordance with ISO guidelines. Additional validity checks were performed to improve the test setup and further increase the level of confidence in the experimental results. The natural frequencies found were compared to the computational model, and where necessary, recommendations for future modelling improvements were made. In the case of the first sub-assembly, discrepancy in natural frequency for certain modes was reduced from $12 \%$ to less than $2 \%$. An acceptable level of correlation was found between experimental and computational results for the second fuselage structure, with a discrepancy of less than $10 \%$ observed for most natural frequencies. Certain natural frequencies showed variance above $30 \%$, and potential reasons for this discrepancy are discussed. The experimental results also provided a basis of comparison for the mode shapes predicted in a computational model constructed by other researchers, acting to further validate computational results. With a detailed experimental methodology formed, and reliable experimental results produced, the project can now progress to the next phase which involves the construction and testing of a complete half scale tail section.
\end{abstract}




\section{Acknowledgements}

First and foremost, I would like to extend my gratitude to my supervisor Dr. Chris Mechefske for his continued technical guidance and support throughout this project. The completion of this work would not be possible without his commitment.

To all my colleagues in the Systems Dynamics Research group, I would like to thank you for your technical support and friendship over the past two years. A special thank you to Diego Chamberlain for forming the groundwork of this project, and for providing a comprehensive introduction to experimental modal analysis. Additionally, I would like to thank Braden Warwick and Chris Lam who in addition to providing computational data, assisted me throughout the various phases of the project.

I would like to thank Stephen Colavincenzo and Reza Madjlesi of Bombardier Aerospace for their continued financial and technical support. Additionally, for providing me the opportunity to work at Bombardier, the experience from which will prove invaluable in the future.

Finally, I would like to thank my close friends and family for their unwavering support throughout my time at Queen's. To my partner, Madeleine, thank you for your continuous support throughout my academic career. To my parents, thank you for everything. Your continuous support throughout my academics and beyond is appreciated more than you know. 


\section{Table of Contents}

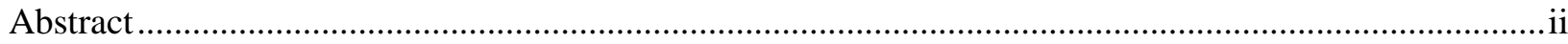

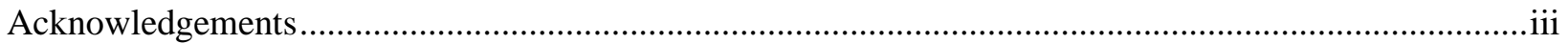

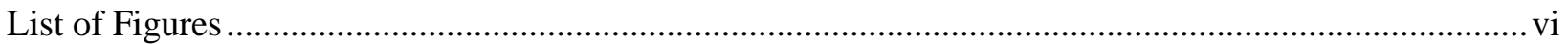

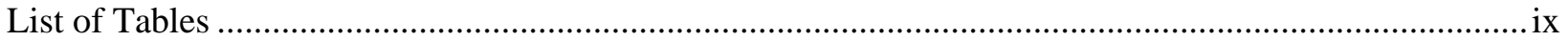

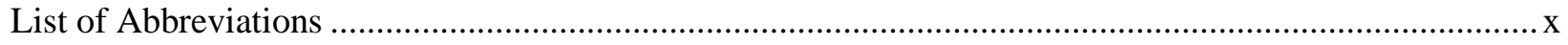

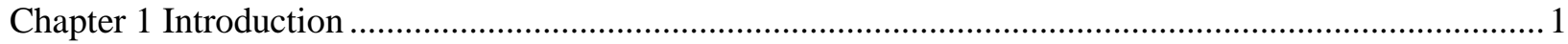

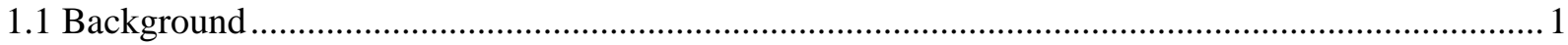

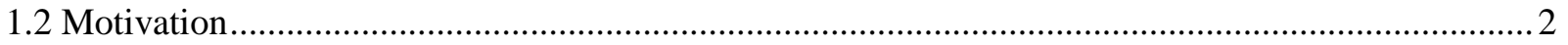

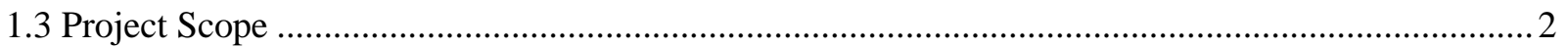

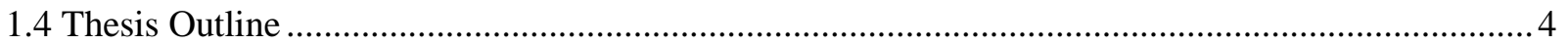

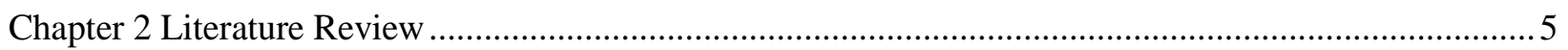

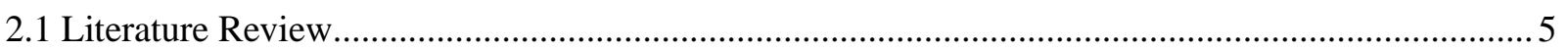

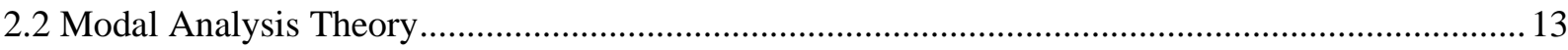

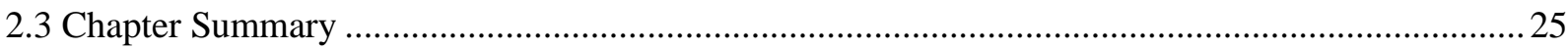

Chapter 3 Fuselage Sub-Assembly Design and Construction..............................................................2

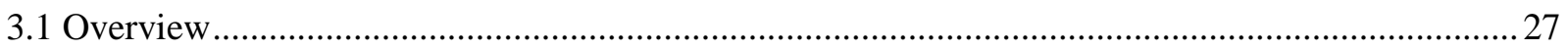

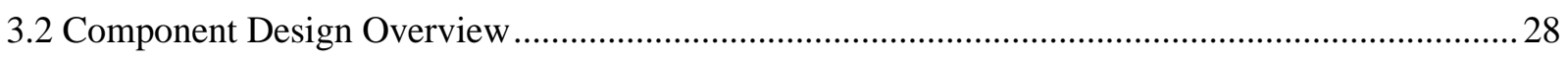

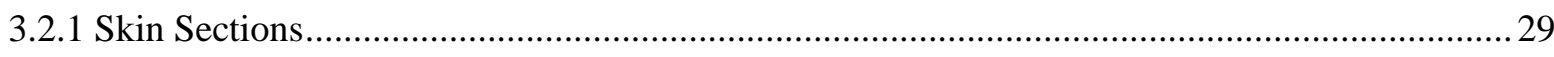

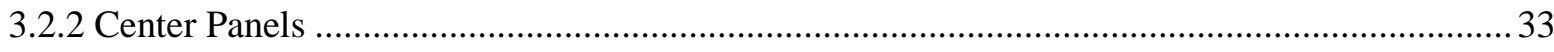

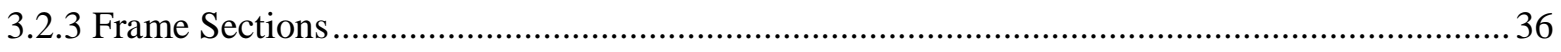

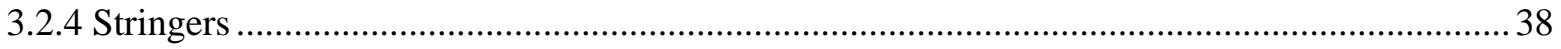

3.3 Sub-assembly Design and Construction ……........................................................................... 39

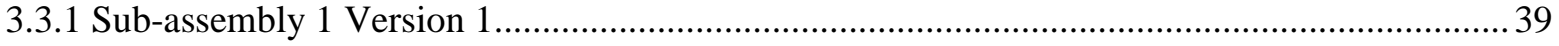

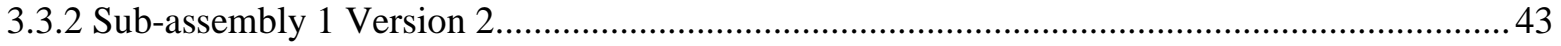

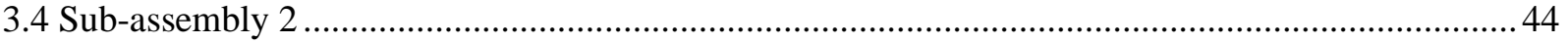

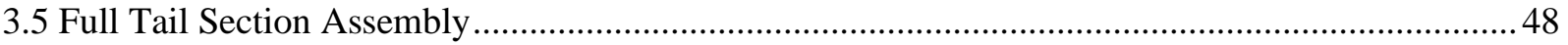

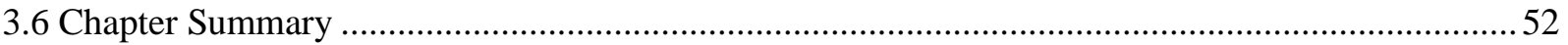

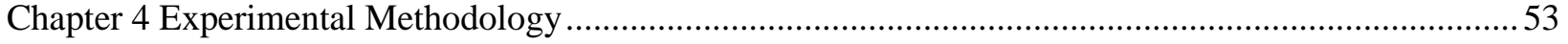

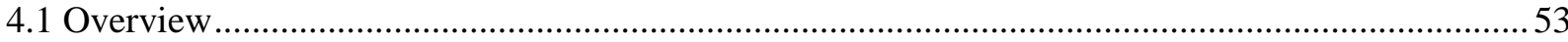

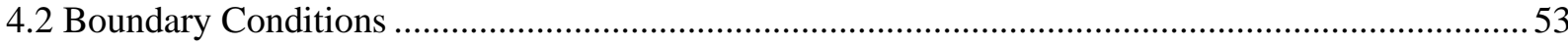

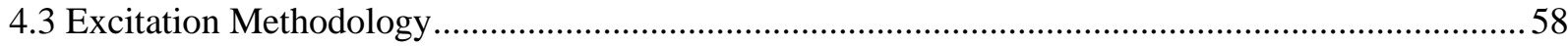

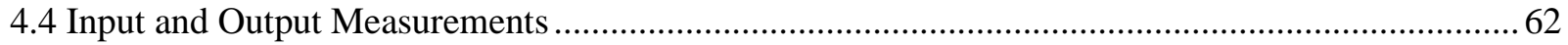




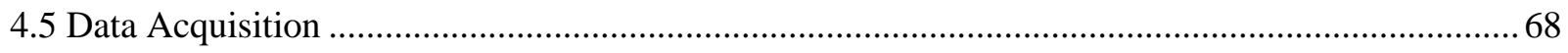

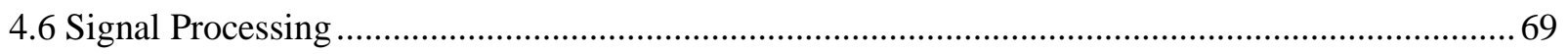

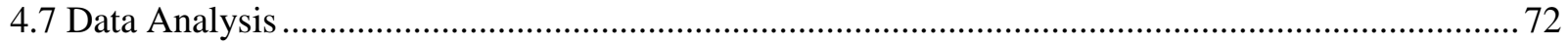

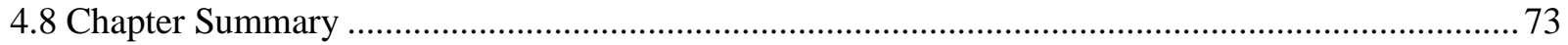

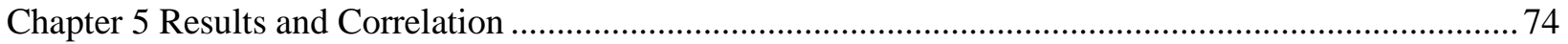

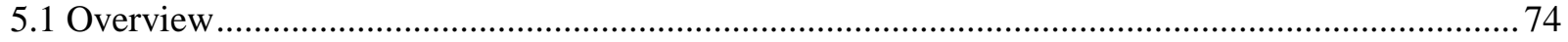

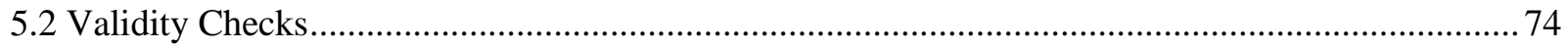

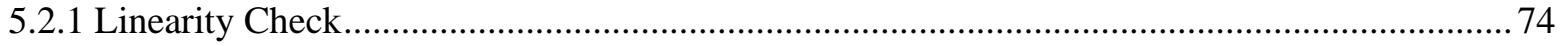

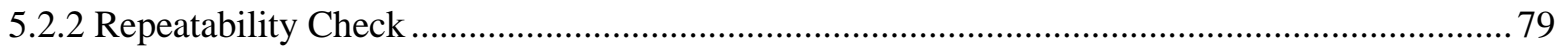

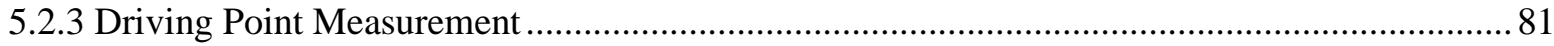

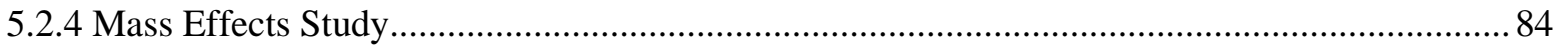

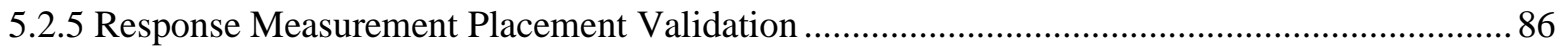

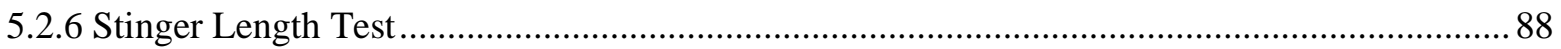

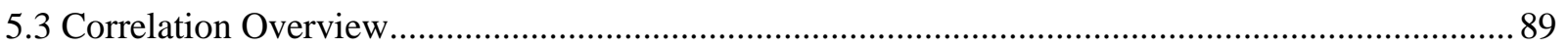

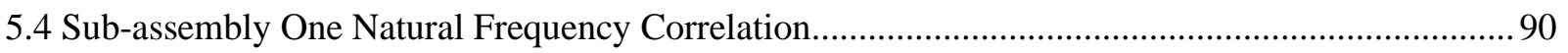

5.5 Sub-assembly One Mode Shape Correlation ............................................................................. 91

5.6 Sub-Assembly Two Natural Frequency Correlation..................................................................... 97

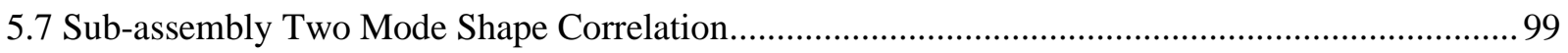

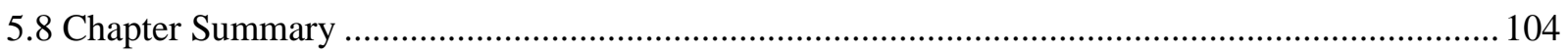

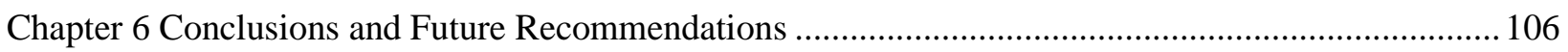

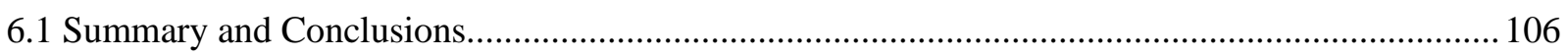

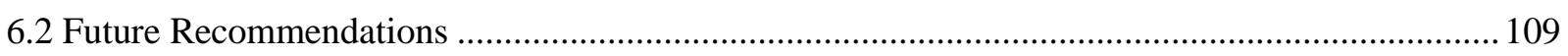

Appendix A Sub-assembly One Experimental Mode shapes .............................................................. 110

Appendix B Sub-assembly Two Experimental Mode Shapes ............................................................... 116

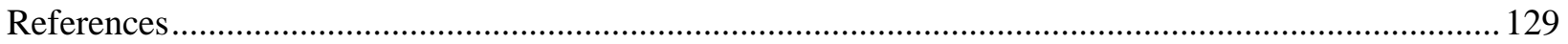




\section{List of Figures}

Figure 2.1: Bode diagram for a SDOF dynamic system (Avitable, 2018).

Figure 2.2: Co-Quad diagram containing real and imaginary parts of an FRF from a SDOF dynamic

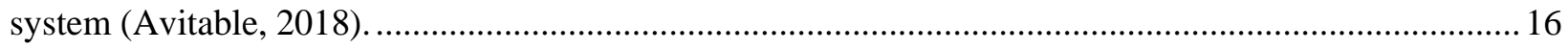

Figure 2.3 A generic mobility plot for a MDOF system. (Ewins, 2000) …............................................23

Figure 2.4:Typical driving point measurement for sub-assembly 1 ......................................................24

Figure 3.1: Schematic for the flat pattern of a truncated cone used for the skin of the first sub-assembly.29

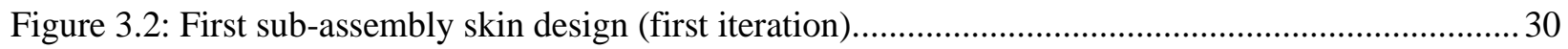

Figure 3.3: First subassembly skin flat pattern (final iteration) .......................................................... 31

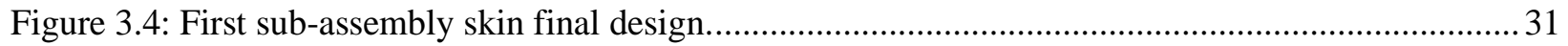

Figure 3.5: Schematic of the second sub-assembly skin, with pylon cut-out detail. ................................ 32

Figure 3.6: The result of bending the flat pattern in Figure 3.5 into the final conical shape...................... 33

Figure 3.7: Schematic of the first sub-assembly aft bulkhead. .............................................................. 34

Figure 3.8: Final schematic for the front engine support frame (FESF) including engine pylons.............. 35

Figure 3.9:Final schematic for the rear engine support frame (RESF) including engine pylons................36

Figure 3.10: Universal cross section schematic used for all frame sections from both sub-assemblies..... 37

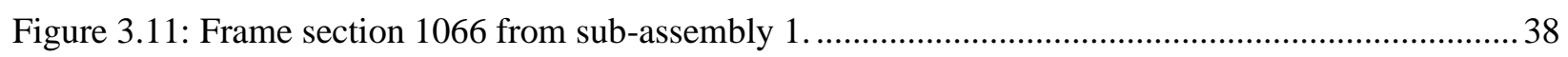

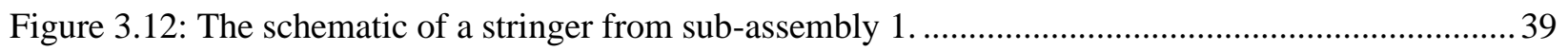

Figure 3.13: A side view of the full tail section showing where sub-assembly 1 originates. .................... 40

Figure 3.14: Isometric view of the full tail section showing where sub-assembly 1 originates. ................40

Figure 3.15: An image showing the completed first version of sub-assembly 1 . .....................................42

Figure 3.16: An image highlighting the poor mechanical connection between sections of skin on the first

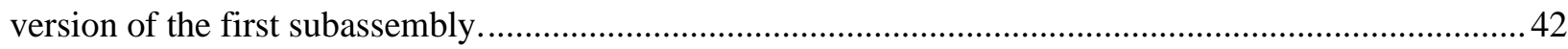

Figure 3.17:An image highlighting the improvement in mechanical connection between sections of skin

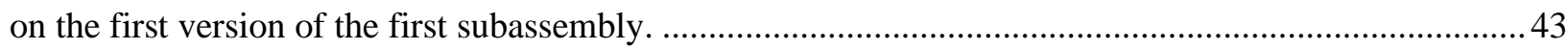

Figure 3.18: A side view of the full tail section showing where sub-assembly 2 originates .....................44

Figure 3.19: Isometric view of the full tail section showing where sub-assembly 2 originates. ............... 44

Figure 3.20: Initial Stages of sub-assembly 2 construction. In this image 3 of 4 frame sections including

both engine support frames have been attached to one panel of skin..................................................... 46

Figure 3.21: The second sub-assembly nearing completion. The slots in the skin form clearance holes for

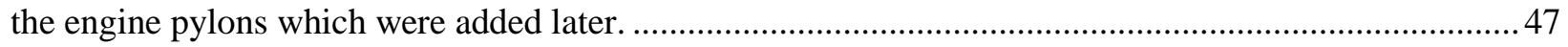

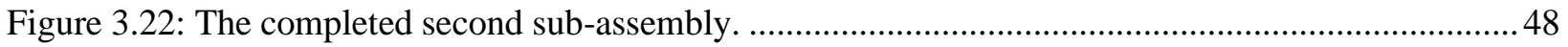


Figure 3.23: The rectangle highlights the frame added to the front of the bulkhead to facilitate the

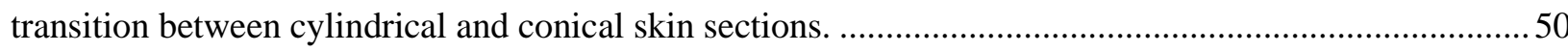

Figure 3.24: Side view of the completed design of the full tail section..................................................50

Figure 3.25: Schematic of the internal structure of the full tail section.................................................51

Figure 3.26: Completed design of the full tail section sub-assembly....................................................51

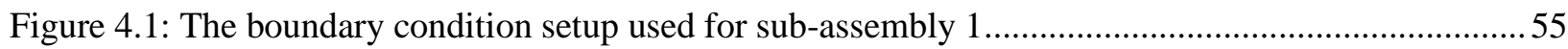

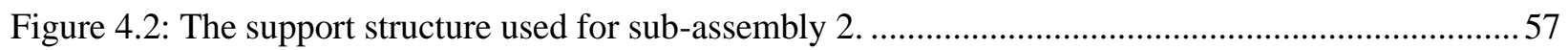

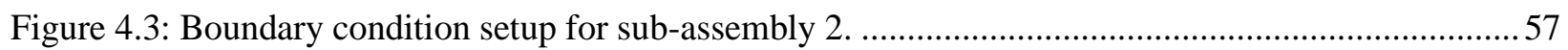

Figure 4.4: Shaker setup during sub-assembly 2 modal testing ...........................................................59

Figure 4.5: Typical drill rod stinger setup implemented during preliminary testing of sub-assembly 1 ....62

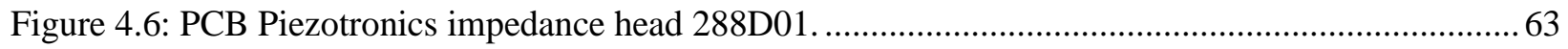

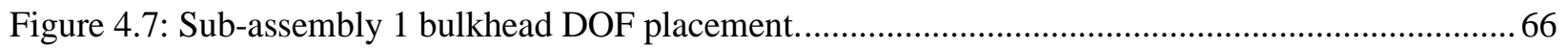

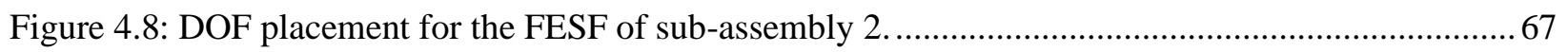

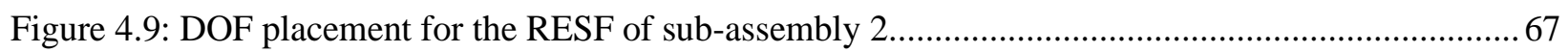

Figure 4.10: Typical FRF and coherence measurement. Notice the dips in coherence in relation to the anti-resonances of the FRF (Avitable, 2018).

Figure 5.1: Linearity check from DOF 2 on the bulkhead of sub-assembly 1. (Orange 2N, Blue 7N and Green $15 \mathrm{~N}$ peak input)

Figure 5.2: Linearity check from DOF 2 on the bulkhead of sub-assembly 1. (Orange 2N, Blue 7N and Green $15 \mathrm{~N}$ peak input)

Figure 5.3: Linearity check from DOF 44 on the FESF of sub-assembly 2. (Orange 3N, Blue 10N and Green 20N Peak Input)

Figure 5.4: Linearity check from DOF 144 on the RESF of sub-assembly 2. (Orange 3N, Blue 10N and

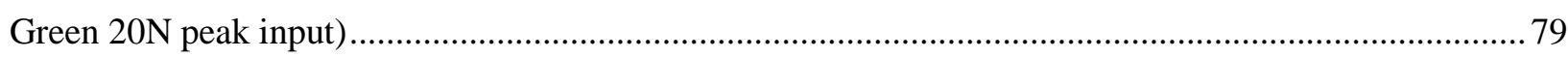

Figure 5.5: Repeatability check of DOF 141 on the bulkhead of sub-assembly 1 .................................... 80

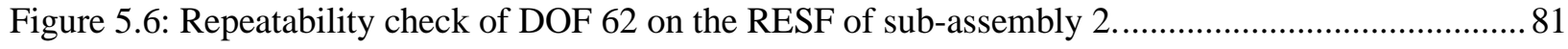

Figure 5.7: Driving point measurement for sub-assembly 1 (Magnitude) …......................................... 82

Figure 5.8: Driving point measurement for sub-assembly 1 (Imaginary) ...............................................8 83

Figure 5.9: Driving point measurement for sub-assembly 2 (Magnitude) …............................................. 83

Figure 5.10: Driving point measurement for sub-assembly 2 (Imaginary) ............................................. 84

Figure 5.11: Overlayed FRF curves for bulkhead of sub-assembly 1 (No Dummy Masses) ....................85

Figure 5.12: Overlayed FRF curves for bulkhead of sub-assembly 1 (With Dummy Masses) .................86

Figure 5.13: Aft Bulkhead AutoMAC before (Left) and after (Right) points were added. ....................... 87

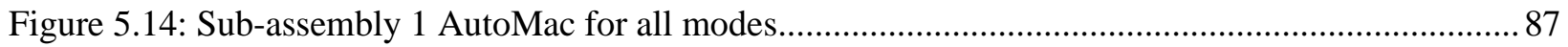


Figure 5.15: Sub-assembly 2 AutoMAC for the FESF (Left) and RESF (Right)

Figure 5.16: Stinger length comparison for DOF 42 of sub-assembly 1. (Orange: $10.5 \mathrm{~cm}$, Green: $15 \mathrm{~cm}$,

Blue: $25 \mathrm{~cm})$ 89

Figure 5.17: Repeated modes of axisymmetric structures (Ewins, 2000). .92

Figure 5.18: "6 Point Star Skin” FEM mode shape (CMP 10 at 172.78 Hz) (Lam \& Mechefske, 2020) .. 93

Figure 5.19: "6 Point Star Skin" Experimental mode shape (CMP 10 at $170 \mathrm{~Hz}$ ) (Lam \& Mechefske,

2020) 94

Figure 5.20: Experimental mode eight at $138 \mathrm{~Hz}$ (highlighted in yellow in Table 1) .95

Figure 5.21: Computational Modes eight and 9 (highlighted in yellow in Table 1) (Lam \& Mechefske, 2020) 95

Figure 5.22: POC computation for 11 correlated modes of sub-assembly one. .96

Figure 5.23: Third FESF experimental mode shape at $41.6 \mathrm{~Hz}$. 100

Figure 5.24: Third FESF computational mode shape at 30.24 Hz (Lam \& Mechefske, 2020). 100

Figure 5.25: Fifth RESF experimental mode shape at $89 \mathrm{~Hz}$ 101

Figure 5.26: Fifth RESF computational mode shape at 85.05 Hz (Lam \& Mechefske, 2020). 101

Figure 5.27: POC computation for 15 correlated modes from the FESF of sub-assembly 2. 102

Figure 5.28: Similarity between FESF mode 11 (Left) and FESF mode 12 (right) found experimentally.

Figure 5.29: Predicted FESF modes 11 (Left) and 12 (Right) (Lam \& Mechefske, 2020). 103

Figure 5.30: POC computation for 10 correlated modes from the RESF of sub-assembly 2. 104 


\section{List of Tables}

Table 5.1: Comparison of sub-assembly 1 natural frequencies ........................................................... 91

Table 5.2: Comparison of sub-assembly 2 natural frequencies 


\section{List of Abbreviations}

Sound Pressure Level - SPL

Experimental Modal Analysis - EMA

Fast Fourier Transform - FFT

Electro-Optic Holography - EOH

Scanning Laser Doppler Vibrometer - SLDV

Finite Element Model - FEM

Multiple Input Multiple Output - MIMO

Ground Vibration Test - GVT

Computational Modal Analysis - CMA

Correlated Mode Pair - CMP

Single Degree of Freedom - SDOF

Frequency Response Function - FRF

Multiple Degrees of Freedom - MDOF

Degree of Freedom - DOF

Unit Modal Mass - UMM

Modal Assurance Criterion - MAC

Pseudo Orthogonality Check - POC

System Equivalent Reduction Expansion Process - SEREP

Computer Aided Machining - CAM

Computer Numerical Control - CNC

Engine Support Frame - ESF

Front Engine Support Frame - FESF

Rear Engine Support Frame - RESF

Rigid Body Mode - RBM

Complex Mode Indicator Function - CMIF

Multivariate Mode Indicator Function - MMIF 


\section{Chapter 1}

\section{Introduction}

\subsection{Background}

With regards to business jets, there is a significant premium placed on aircraft that provide a luxurious flying experience. Noise in aircraft is an ever-present issue which negatively affects the comfort and overall flying experience for passengers. Jet engines produce a significant portion of the noise which is transmitted to the interior along airborne or structure borne paths (Mixson \& Kearny, 1978). This noise is generated from vibrations induced from mechanical imbalances in the engines, and other more complex mechanisms such as exhaust interactions with the outside of the plane. For aircraft with wing mounted engines the airborne transmittance of sound is the primary concern, and for aircraft with fuselage mounted engines, structure borne noise is more prevalent (Wilby, 1995). This research aims to reduce the sound pressure level (SPL) in a range of business jets with fuselage mounted jet engines produced by Bombardier Aerospace, by first obtaining an understanding of the dynamic characteristics of a generic representation of the tail section of these aircraft. In future this understanding will guide structural modifications to alter the dynamic response of the fuselage tail section and reduce interior cabin noise.

Experimental modal analysis (EMA) is often used to understand the vibrational characteristics of structures. Modal parameters found using EMA such as the natural frequencies and mode shapes are often compared to a computational model for validation. Once it is shown that the computational model can accurately represent the dynamic properties of a structure, improvements can be proposed, tested computationally, and finally tested experimentally. These improvements can benefit a system in a variety of ways, and for this project they aim to reduce the dynamic response of the system resulting from an excitation from the primary engine tone of 
$117 \mathrm{~Hz}$. This methodology significantly reduces the amount of physical testing required, and the cost associated with making multiple structural modifications.

\subsection{Motivation}

The primary motivation behind this research is to improve passenger comfort for those flying in the newest generation of business jet. These aircraft are designed according to multiple design objectives including performance, fuel economy and passenger comfort. The new generation of business jets offer faster cruising speed, and longer overall range; however they struggle to provide a quiet environment associated with a luxurious flying experience. In this highly competitive sector of the market, it is important to gain an advantage wherever possible. Previous research has been performed to relate passenger discomfort to interior vibration and sound pressure level. Dempsey et al. have shown that passenger discomfort is linearly related to vibration acceleration level and varies logarithmically with interior sound pressure level (Dempsey et al. 1978). To gain an advantage with respect to comfort, reducing the interior sound is paramount. As such there is a strong motivation to understand the dynamic response of business jet fuselage structures, such that improvements can be designed, validated, and implemented. These improvements will aim to reduce noise with a minimal impact on other design objectives. The first step, however, is to understand the dynamic response of a generic aircraft fuselage to form a foundation of knowledge that will be used to guide these future improvements.

\subsection{Project Scope}

A prominent Canadian aircraft manufacturer has shown interest in reducing noise and vibration in the passenger cabin for a range of their business focused aircraft. To accomplish this, the dynamic response of the fuselage of the aircraft must be understood, such that measures can be implemented to mitigate structure borne noise and vibration. A significant portion of the interior SPL was found to be generated by the fuselage mounted jet engines. This is in accordance 
with research performed by Wilby which found that aircraft with fuselage mounted engines were far more susceptible to structure borne noise propagation through the fuselage into the passenger cabin (Wilby, 1995).

Interest has been focused on the tail section of the aircraft where both jet engines are mounted, with previous research performed by Chamberlain on individual half scale fuselage components. This research was focused on understanding the dynamic response of these components using EMA and provided a source of validation for computational work performed by Warwick (Chamberlain, 2018) (Warwick, Mechefske, \& Kim, 2018). This work formed the first stage of a bottom up approach which will be implemented across multiple research projects. Chamberlain also devoted time to design a complete half scale fuselage tail section. This model was designed to be generic in nature, such that the results from both the experimental test and computational model would indicate general trends, and the findings from this half scale model will be applicable to a wider range of fuselage tail sections.

This thesis aims to satisfy two primary objectives. The first of which is to provide detailed and reliable experimental results for the purposes of computational model validation. The second objective is to form a comprehensive experimental methodology which will be applicable throughout the next phase of the project on a larger fuselage structure.

To satisfy these objectives, the research presented in this thesis focuses on expanding the work performed on individual components, to half scale fuselage tail section sub-assemblies. It will provide an intermediate step between the tests performed previously, and the full half scale tail section experiments to be performed in future. In addition to his work modelling individual components, Warwick et al. have also investigated these components as part of larger subassemblies (Warwick, Kim, \& Mechefske, 2019). As a result, experimental data was required to form a basis of comparison to validate the computational models of fuselage sub-assemblies. To satisfy this requirement, this thesis focuses on the experimental testing of two fuselage tail section 
sub-assemblies. The research presented will help validate Warwick's work and inspire updating techniques to improve the accuracy of other computational models created by Lam et al. (Lam \& Mechefske, 2020). The experiments performed during this project will also form a testing methodology suitable for EMA on aircraft fuselage sub-assemblies.

\subsection{Thesis Outline}

This thesis provides a complete overview of the research, experimental methodology, and results associated with the experimental modal analysis of aircraft fuselage sub-assemblies. To accomplish this, it has been divided into 6 chapters. Chapter 2 provides an overview of modal analysis theory relevant to this project. Additionally, a review of previous research performed with regards to modal analysis in aerospace is presented. Chapter 3 provides a detailed description of both sub-assemblies. This includes an overview of their design, as well as an indepth description of the manufacturing methods chosen for the completion of both fuselage sections. Finally, recommendations are made for future construction of the complete half-scale tail section assembly. Chapter 4 describes the experimental methodology used to extract the modal parameters from both sub-assemblies. The experimental setup, excitation method, measurement location, and data acquisition parameters are covered in detail, as well as a detailed account of the checks used to validate the experimental data. The results from these experiments are presented in chapter 5 . This chapter also discusses the correlation between the experimental data and the computational predictions generated by Lam; whose work is cited where appropriate. The natural frequencies as well as the mode shapes are compared, and discrepancies between the two data sets are discussed. Future testing improvements, as well as a detailed description of future work are outlined in chapter 6 . This chapter also presents conclusions and a summary of the work presented. Any figures deemed unsuitable for placement in the body of this thesis are placed in the appendices and are referenced where appropriate. 


\section{Chapter 2}

\section{Literature Review}

\subsection{Literature Review}

The era of modern modal analysis can be dated back to the 1960's, when the field began to branch out from purely research applications into commercial avenues. This expansion was predicated by developments such as the Fast Fourier Transform (FFT) developed by Cooley and Tukey in 1963, as well as advances in sensors and data acquisition technology (Brown \& Allemang, 2007).These innovations helped make experimental modal analysis (EMA) accessible to a wide range of industries looking to understand the dynamic characteristics of mechanical systems. Soon after this transition into industry, EMA found its way into aerospace, and ever since aircraft manufacturers have used experimental modal analysis to develop their product. Many examples of such experiments can be found in the literature, and the following section will provide a review of this content. Previous work has shown the applicability of EMA on a wide range of aircraft fuselage components and sub-assemblies and modal analysis has also been implemented as part of aircraft certification procedures in the form of ground vibration tests (Goge, Boswald, Fullekrug, \& Lubrina, 2007). EMA has also acted as a source of validation for computational models and has inspired improvements in modelling techniques (Lam \& Mechefske, 2020). Additionally, measurement techniques used in EMA are constantly improving, and these developments have been implemented in aerospace to integrate modal analysis techniques as part of structural fault detection systems in aircraft.

An early implementation of EMA documented by Gordon et al. is used to investigate the modal parameters of lightweight aircraft structures (Gordon, Wolfe, \& Talmadge, 1977). The primary objective behind this research was to obtain a more complete understanding of the fatigue life of aerospace components due to dynamic loading under operating conditions. To 
accomplish this, it was deemed necessary that the dynamic properties of the components in question be understood, such that new failure modes and high stress locations could be discovered. Another motivation of this project was to evaluate the effectiveness of a novel testing method (for the time) which involved the use of an impact hammer, and a digital approach to data analysis and manipulation. This research is documented as an early example of the use of digital methods including a minicomputer which facilitated the use of the recently discovered Fast Fourier Transform. As such, it was important to prove its viability through comparison with wellestablished methods.

During the experiment, three aircraft panels were tested in two separate trials. The first used a well-established analog testing methodology which employed a speaker for excitation using a swept sine input signal, and accelerometers to measure the dynamic response. The digital methodology used an impact hammer to supply a random excitation, and a minicomputer in conjunction with an FFT algorithm to collect and transform the data into the frequency domain. The results from both tests were compared to evaluate discrepancies between analog and digital test methods, and modal parameters including the natural frequencies and modes shapes of each panel were found. It was shown that the digital modal analysis provided consistent results with those produced by the previously well-established analog techniques. Also noted by (Gordon, Wolfe, \& Talmadge, 1977). were the improvements in testing associated with the impact hammer methodology. These included the vastly expedited set-up and test time, the reduction of hazards associated with noise, and the increased flexibility of data storage and manipulation associated with digital output. Limitations of the method are also indicated and include the limited range of excitation, but improvements to the impact hammer technology, as well as developments with modal shakers would remedy these issues.

Other experiments have been performed on stiffened aircraft panels by Fleming et al. involving the extraction of modal parameters using electro-optic holography $(\mathrm{EOH})$ as well as a 
scanning laser doppler vibrometer (SLDV) on a full-scale fuselage panel. The panel constructed was designed as a general representation of an aircraft fuselage skin section, with stringers and frames attached to simulate stiffening structures present on aircraft. One of the main issues with the EOH measurement method is the corruption of high frequency mode shapes from large amplitude rigid body modes (Fleming \& Buerhle, 1998). The development of image processing algorithms to allow for a free-free boundary condition in the experimental setup is presented in this work, as well as the validation of finite element models (FEM) using the experimental data from both measurements.

One of the main focuses of this work was to improve the quality of interferogram output from the EOH measurement. The initial measurements proved unsuccessful, as they did not provide a clear image of the various mode shapes, with issues including poor contrast and distortion caused by unrelated rigid body motion. To improve image quality, enhanced interferograms were constructed using ensembles of 64 interferograms at each resonant mode shape. An algorithm was then employed to guide this construction, using regions within the ensemble having increased contrast and brightness. This method proved to be successful in providing a clear image of each mode shape, while minimizing distortion from rigid body motion. The enhanced results from the EOH measurements were compared to those generated by the SLDV method, and the natural frequencies and mode shapes from both sets showed strong correlation.

Modal parameters including the natural frequencies and mode shapes collected from both experimental data sets were used to guide modifications to the FEM to improve correlation. Three separate models using different element type configurations to construct the skin stiffeners were evaluated. These configurations included using beam elements, plate elements, then a hybrid of the two to account for varying stiffener geometry. The configuration that provided the best correlation with the experimental results was the plate stiffener model, which most closely 
followed the trends in natural frequency found in the experimental data. The computational model still underpredicts natural frequency, and work is ongoing to improve correlation.

Another example of EMA being used to determine the modal characteristics of a stiffened aircraft panel was documented by Wyen et al. (Wyen, Schoettelkotte, Perez, \& Eason, 2017).This work formed a portion of a detailed testing methodology developed by the Air Force Research Laboratory. The objective of this project was to improve the design process of hypersonic aircraft components subject to complex loading conditions, and integral to this was the determination of the dynamic properties of an aircraft fuselage panel under different operating conditions. Two experimental approaches were used over four separate modal tests to evaluate changes in modal parameters due to the addition of instrumentation and structural members. A preliminary test utilized a roving impact hammer technique to find a preliminary set of natural frequencies, mode shapes and damping values of the fuselage panel with structural members called load introduction fittings. This test also provided a preliminary basis of comparison for a FEM, and the discovered mode shapes in conjunction with the computational model helped guide the placement of response measurement locations for future experiments. The second experiment used three modal shakers to provide excitation, and laser doppler vibrometers to measure the response of the same structure in three dimensions. The results from these tests were compared, and minimal discrepancies were noted, indicating that both methodologies provided reasonable results. The third test used the same multiple input multiple output (MIMO) methodology to test the changes in modal characteristics resulting in the removal of load introduction fittings from the fuselage panel. These fittings had a stiffening effect on the perimeter of the structure, and as expected local modes near this part of the panel occurred at lower frequencies during this test. The final test was conducted using a roving impact hammer on the fuselage panel with attached load introduction fittings, as well as a full suite of other instrumentation necessary for other experimentation involved with the project. This test was performed to provide an insight to the 
effects of the attached instrumentation on the modal properties of the fuselage panel in operation. It was found that the attached instrumentation increased the damping factor and decreased the natural frequency of all modes. In summary, the work presented provides a detailed account of two modal test methodologies used to determine the modal properties of a stiffened fuselage panel. The experimentally determined properties were then used to update the computational model, to improve its representation of the fuselage panel under operating conditions.

The applicability of EMA has also expanded outside the field of research and development, into aircraft certification procedures in the form of ground vibration tests (GVT). The primary purpose of a GVT is to identify "flutter" modes of an aircraft which can have catastrophic structural effects on the aircraft during operation. "Aeroelastic flutter involves the unfavorable interaction of aerodynamic, elastic, and inertia forces on structures to produce an unstable oscillation that often results in structural failure." (Kehoe, 1995)

To accomplish this modal shakers and hundreds of response transducers are often required, resulting in a complex experimental procedure that can often take weeks to complete. The results from these tests are then used to update the computational model, which once validated, can predict flutter critical speeds. Therefore, the production of accurate results from the GVT is essential to provide accurate predictions of flutter during flight. Recent developments in the experimental procedure with regards to GVT's are documented by (Goge, Boswald, Fullekrug, \& Lubrina, 2007). In addition to recommendations regarding implementation of modern transducers (wireless accelerometers for example), a new process for the verification of aeroelastic models was proposed to further expedite the experimental process. Procedural modifications included testing critical components and substructures in parallel with aircraft development. This would allow for the modal parameters of critical structures to be defined prior to a full GVT. Additionally, the experimental results from these preliminary tests would provide data for computational model validation. Preforming this work beforehand could allow for a 
reduced GVT on the completed aircraft during the certification process, thus reducing overall production time.

Changes in the dynamic characteristics of components can often indicate the presence of a structural fault in aircraft, otherwise undetectable through other investigation techniques. Modal analysis procedures are often used to detect faults which would manifest as a change in the modal properties of a component or structure. The integration of accelerometers or other transducers often used in EMA, is often not possible for structures in operation. A measurement system which can be easily integrated into aircraft structures is desirable to accurately indicate changes in structural integrity. (Cusano, et al., 2006) describe the integration of Bragg grating sensors into a model wing for the purposes of EMA. The objective of their experiment is to compare the data output from these fiber optic strain sensors with data output from sensors more conventionally used in EMA to demonstrate the applicability of this measurement method.

To evaluate the validity of this new transducer arrangement, EMA was performed on two identical composite wing structures. These wings formed approximations of what might be found on unmanned aircraft. One was constructed to be tested using accelerometers, and the other integrated fiber Bragg grating sensors within the main structural member of the wing. EMA was performed on both wings over a $0-140 \mathrm{~Hz}$ bandwidth, using a roving impact hammer for excitation. As opposed to a free-free boundary condition, a rigid support was implemented on one end of each wing to simulate the structural support provided by the fuselage of the aircraft. It was found that both measurement techniques provided a complete set of mode shapes over the frequency range of interest, and minimal discrepancies in the natural frequency of each mode were found. As such, it has been shown that this "resident health monitoring system" provides a complete and accurate set of results with respects to EMA. Next steps could include the integration of this measurement system into a wide range of composite structures for damage detection during operation using the core principles of EMA. 
Prior to the work presented in this thesis, EMA was conducted on multiple half scale fuselage components, and this work is presented in detail by Chamberlain et al. at Queen's University (Chamberlain, 2018). The primary objective of this project was to understand the dynamic response of fuselage structures, such that structural modifications could be proposed to reduce interior sound pressure level on business aircraft. The work presented focuses on the pressurized bulkhead and engine support frame panels. These components were selected due to their proximity between the fuselage mounted jet engines and the passenger cabin, and their direct effect on the structure borne noise propagation through the tail of the aircraft. During the design process, general simplifications were made to enable the construction of such components with the available resources. As a result, the experimental results would not provide an exact representation of the dynamic properties of components on production aircraft. Instead general trends in the dynamic response could be discovered, and in future design modifications will be proposed and evaluated. Additionally, the experimental results provided in this study serve to validate computational models and where necessary, provide guidance for model updating techniques.

To complete this study, three pressurized bulkheads with different stiffener arrangements and two engine support frames were designed and assembled using general aviation practices for inspiration. Implementing different stiffener geometries on the pressurized bulkhead panels provided an indication of the effects of bending stiffness on natural frequencies and mode shapes of the panel. Creating three different bulkheads also provided a larger set of experimental results to aid with model updating and validation. The engine support frames form the most direct structural connection between the jet turbines and the fuselage, and as such, a detailed understanding of their respective dynamic properties was required, before integrating them into a full tail section model. 
To obtain the modal characteristics of each component, impact hammer testing was implemented in accordance with modal testing best practices. Validity checks including reciprocity, linearity, and repeatability checks were found to be essential for ensuring the reliability of data output from an impact hammer modal test (Chamberlain, 2018). Other guidelines found in ISO 7626 including the averaging procedures used during a modal test were also recognized and followed rigorously (International Organization for Standardization, 1994). The adherence to EMA best practices during this study provided high quality experimental results and a reliable source of validation for the computational model.

A proposed outline of future work is also provided and describes potential next steps for the project. These steps include expanding experimental modal testing on more complex fuselage sub-assemblies to form an intermediate step between the individual components and the full tail section model. Additional proposed steps involve investigating developments in test methodology, including the implementation of a modal shaker for excitation, and expanding the frequency bandwidth from $\sim 0-90 \mathrm{~Hz}$ to $0-200 \mathrm{~Hz}$. These recommendations provided a framework on which the experimental methodology described in chapter 4 was created, and a defined objective for the research presented in this thesis.

Although this research is not directly involved with computational modelling of dynamic systems, the results presented provide a source of validation for such models. Recent work with computational modal analysis (CMA) was performed by Warwick et al. to investigate the effects of stiffener configurations on the modal properties of an aircraft pressurized bulkhead (Warwick, Mechefske, \& Kim, 2018). This paper outlines a methodology by which computational and experimental results can be compared and correlated. This methodology includes the qualitative correlation of mode pairs, the comparison of natural frequency of the correlated mode pairs (CMP), and the quantification of correlation using the modal assurance criterion and pseudo orthogonality checks. Using this methodology, the computational model was validated using 
experimental results provided by (Chamberlain, 2018), and then developed to include operational boundary conditions through the implementation of a frame structure around the bulkhead perimeter. Both sets of results showed strong correlation and indicated that the stiffener configuration had a significant effect on natural frequencies and modal density over certain frequency bandwidths. Low frequency modes were more effected by the mass of added stiffeners, while the frequency of modes higher in the bandwidth was increased as a result of the stiffness from the added components. Finally, the author notes the importance of implementing this validation methodology before progressing to more complex models stating "If the fundamental nature of the component and the effect of basic modifications to the component are not well understood, it is impossible to perform advance analysis techniques.” (Warwick, Kim, \& Mechefske, 2019)

\subsection{Modal Analysis Theory}

The following subsection aims to provide an overview of EMA theory relevant to the research performed for the completion of this thesis. It will primarily focus on theory required to perform a successful modal experiment, and where necessary will touch on the theory surrounding dynamic systems. Two textbooks were referenced during the research phase of this project. "Modal Testing- A Practitioner's Guide” by Peter Avitable provides an in-depth description of the procedures to follow for a wide range of modal tests, as well as a brief review of EMA theory. "Modal Testing Theory Practice and Application” by David Ewins provides a more in-depth review of the theory and is of special interest when considering the methodology used to correlate experimental and computational results. Of course, only the necessary sections of theory are presented below, and for a more complete review of EMA theory, the aforementioned text should be reviewed.

To begin, the case of undamped forced vibration should be considered, as it provides a simple mathematical approximation of a structure's dynamic response to a certain input. As with 
most terms in dynamic equations, the force input will be written in the harmonic form $f(t)=F e^{i \omega t}$ as will the solution $x(t)=X e^{i \omega t}$. We find that the equation for the undamped response of a structure subject to a harmonic input is represented by:

$$
\left(-\mathbf{m} \omega^{2}+\mathbf{k}\right) X \mathrm{e}^{\mathrm{i} \omega \mathrm{t}}=\mathbf{F} \mathrm{e}^{\mathrm{i} \omega \mathrm{t}}
$$

Equation 2.1 for a single degree of freedom (SDOF) system can then be re-arranged to form a ratio of the output response over the input force, which yields the fundamental form of the frequency response function (FRF) for an undamped system.

$$
H(\omega)=\frac{X}{F}=\frac{1}{\left(-\mathbf{m} \omega^{2}+\mathbf{k}\right)}
$$

To represent a dynamic system more completely, a viscous damping term is added to this formula in the form of a complex term in the denominator. This equation forms the general formulation for the frequency response function of a SDOF dynamic system.

$$
H(\omega)=\frac{X}{F}=\frac{1}{\left(-\mathrm{m} \omega^{2}+\mathrm{k}+\mathrm{i}(\omega \mathrm{c})\right)}
$$

In its current form, Equation 2.3 represents the receptance of a structure, or the ratio of the response displacement to the harmonic input force. This ratio is complex, and as such it provides both magnitude and phase information. There are however other types of FRF's which will result from different measurement methods used during a modal test. The first of which is the mobility FRF which is the ratio of response velocity to input force, and the common output of SLDV measurements. The third FRF parameter known as inertance or accelerance is the ratio of response acceleration to input force. This parameter is the output from modal tests using accelerometers for response measurement. This was the method of choice for the experiments presented in this thesis, and as such all experimental results presented are in the form of acceleration / force, or $\frac{\mathrm{m} / \mathrm{s}^{2}}{\mathrm{~N}}$. 
Graphing these complex value functions is not a trivial process. There are three parameters which must be accounted for, including the amplitude, frequency, and phase angle. These values cannot be displayed on a standard x-y graph, and as such there are a multitude of displays to choose from depending on the desired information. Of these, three formats were used when performing data analysis for this project. The Bode plot provides magnitude vs frequency along with phase angle vs frequency in two graphs oriented as shown in Figure 2.1. Notice the phase change that occurs at resonance, as the inertial force surpasses the driving force resulting in a loss of $180^{\circ}$. The bode diagram is the most common form of frequency response plot used during the data analysis phase of EMA (Avitable, 2018).

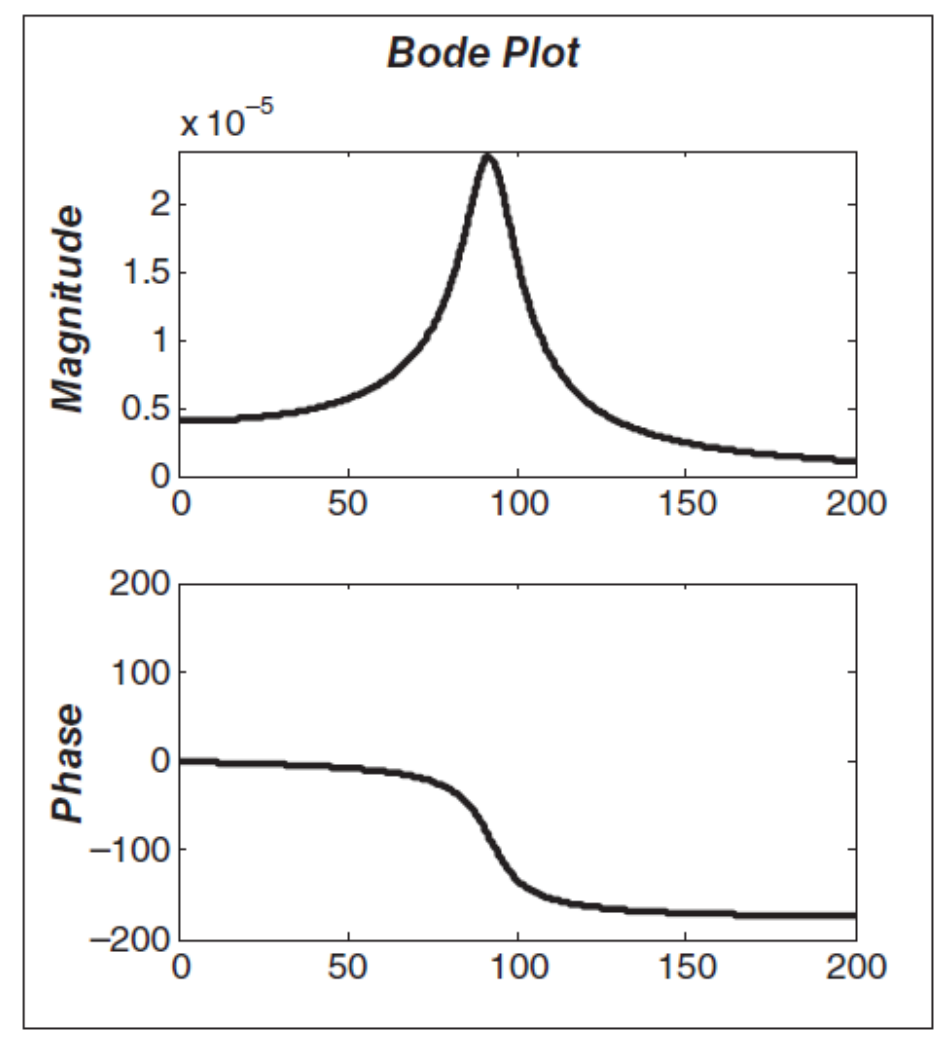

Figure 2.1: Bode diagram for a SDOF dynamic system (Avitable, 2018).

Two other forms of FRF plot are commonly used during analysis and modal parameter extraction. The real and imaginary components of the FRF are plotted against frequency in most cases and provide a clear picture of the resonance peaks in relation to frequency. It is for this 
reason that the imaginary part of the FRF was used during modal parameter extraction, a decision which was inspired by work done previously by Chamberlain. (Chamberlain, 2018). The coincident quadrature or "Co-Quad" plot provides information from the real (coincident) and imaginary (quadrature) parts of the FRF in two graphs oriented in a similar fashion as the phase and magnitude plots in the Bode diagram. An example of a SDOF Co-Quad plot is shown in Figure 2.2. Note how the real part of the function goes to zero at resonance while the imaginary part is a maximum. For this research, modal parameters were extracted using the diagrams described in this section. Other diagrams for displaying EMA data including the Nyquist plot are available, and these are outlined in both the referenced texts.

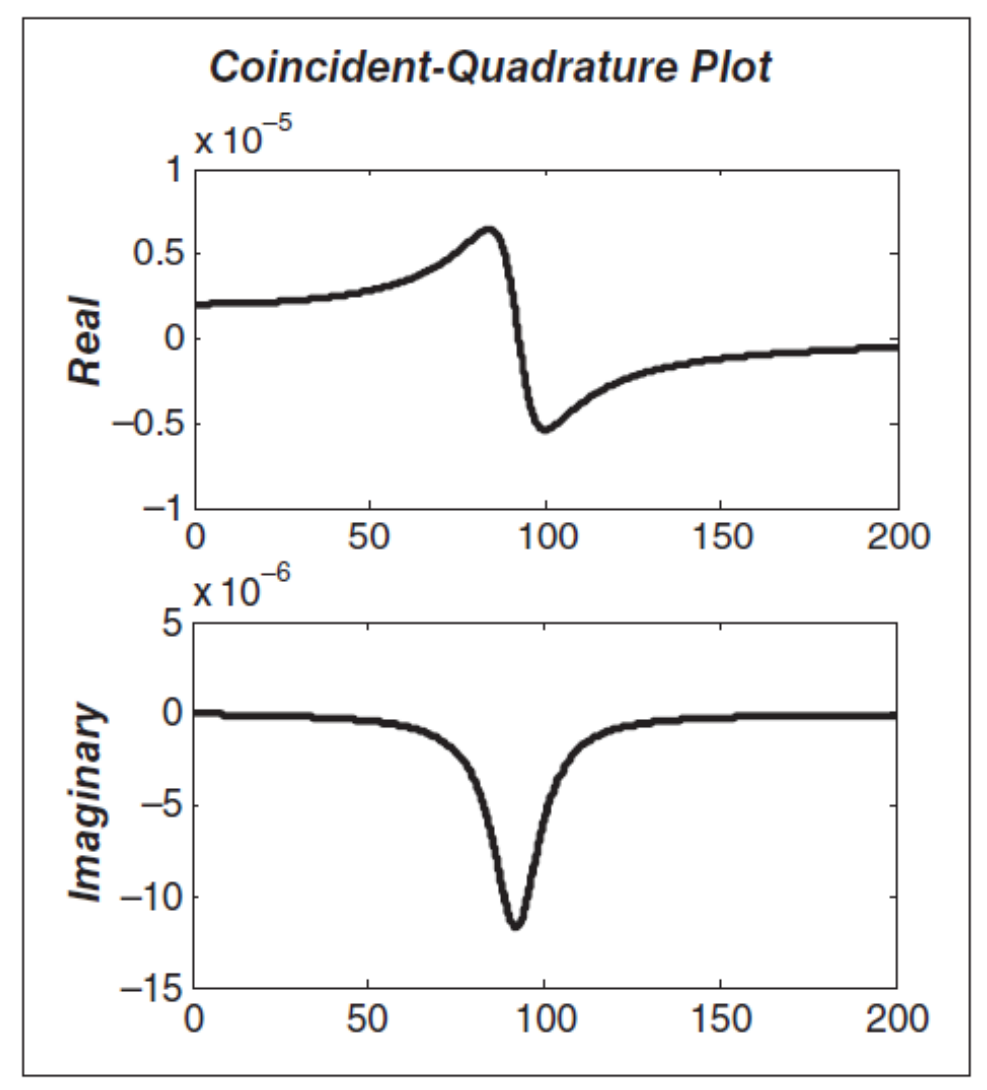

Figure 2.2: Co-Quad diagram containing real and imaginary parts of an FRF from a SDOF dynamic system (Avitable, 2018). 
Equation 2.1 can also be expanded to represent multiple degrees of freedom (MDOF) by implementing matrices to represent multiple mass and stiffness terms, along with vectors to account for motion of multiple bodies. The matrices have dimension N X N, while the vectors are $\mathrm{N}$ terms in length where $\mathrm{N}$ is the number of degrees of freedom (DOF) in the system. For simplicity, an undamped free vibration case will be considered, in which case the force term is zero, however if the external force on the system is known, the math remains largely the same.

$$
\left(-[\mathrm{M}] \omega^{2}+[\mathrm{k}]\right)\{\mathrm{X}\} \mathrm{e}^{\mathrm{i} \omega \mathrm{t}}=\{\mathrm{F}\} \mathrm{e}^{\mathrm{i} \omega \mathrm{t}}=\{0\}
$$

There are two solutions to equation 2.4 for a dynamic system experiencing free vibration. The "trivial" solution represents a system at rest, or in equation form $\{X\}=0$. For the "nontrivial" solution, Equation 2.5 can be used to find values of $\omega$ for each natural frequency of the system.

$$
\operatorname{det}\left|-[M] \omega^{2}+[K]\right|=0
$$

The determinant of this equation is a polynomial of order $2 \mathrm{~N}$ and the roots of this polynomial are the square of the system natural frequencies $\left(\omega_{R}{ }^{2}\right)$. These values can then be substituted into equation 2.4 to find the mode shape vector $\left\{\psi_{R}\right\}$ which is equivalent to the $\{X\}$ term in the equation. As $[\mathrm{M}]$ and $[\mathrm{K}]$ form $\mathrm{N} \mathrm{X} \mathrm{N}$ matrices, it can be seen that the number of solutions to this equation is equivalent to the number of system DOF's. It should also be noted that while the vector of natural frequencies (eigenvalues) is unique, the matrix of mode shapes (eigenvectors) is not, as the mode shapes are subject to some indeterminate scaling factor (Ewins, 2000). In other words, each natural frequency is a fixed value, but each mode can oscillate at any amplitude while maintaining their characteristic shape.

An important property of linear modal analysis is the orthogonal nature of different mode shape vectors. That is, each mode shape vector used to describe the motion of a MDOF dynamic system will be orthogonal to one another. This property is explained mathematically by 
manipulating the equation for free vibration of a MDOF system as shown in the following set of equations. First the equation of motion is evaluated for a particular mode to find equation 2.6.

$$
\left(-[\mathrm{M}] \omega_{R}^{2}+[\mathrm{k}]\right)\{\psi\}_{R}=\{0\}
$$

where $\omega_{R}$ and $\{\psi\}_{R}$ are the natural frequency and mode shape for mode R respectively. Then premultiply this equation by the transpose of a different eigenvector to find equation 2.7.

$$
\{\psi\}_{S}^{T}\left(-[\mathrm{M}] \omega_{R}^{2}+[\mathrm{k}]\right)\{\psi\}_{R}=0
$$

For mode $\mathrm{S}$ the following equation can be written.

$$
\left(-[\mathrm{M}] \omega_{S}^{2}+[\mathrm{k}]\right)\{\psi\}_{S}=\{0\}
$$

This equation can then be transposed, and post multiplied by $\{\psi\}_{R}$.

$$
\{\psi\}_{S}^{T}\left(-[\mathrm{M}]^{T} \omega_{R}^{2}+[\mathrm{k}]^{T}\right)\{\psi\}_{R}=0
$$

Since $[\mathrm{M}]$ and $[\mathrm{k}]$ are generally symmetric and therefore identical to their transposes, equations 2.7 and 2.8 can be combined to form equation $2.10 \mathrm{a}$. It can be seen that this equation is only satisfied under the condition shown in $2.10 \mathrm{~b}$.

$$
\begin{gathered}
\left(\omega_{R}{ }^{2}-\omega_{S}{ }^{2}\right)\{\psi\}_{S}^{T}[\mathrm{M}]\{\psi\}_{R}=0 \\
\{\psi\}_{S}^{T}[\mathrm{M}]\{\psi\}_{R}=0 ; R \neq S
\end{gathered}
$$

For the case where $\mathrm{R}=\mathrm{S}$ the following two equations apply; where $m_{r}$ and $k_{r}$ represent the modal mass and stiffness respectively. These equations also represent a transformation from the physical space to the modal space, which results in diagonal mass, damping and stiffness matrices (Ewins, 2000). The same transformation can be performed on the damping matrix assuming it is proportional to either the mass or stiffness matrices (ideal damping) as shown in 2.13 where $\beta$ is some proportionality constant.

$$
\begin{gathered}
\{\boldsymbol{\psi}\}^{\boldsymbol{T}}[\boldsymbol{M}]\{\boldsymbol{\psi}\}=\left[\boldsymbol{m}_{\boldsymbol{r}}\right] \\
\{\psi\}^{T}[K]\{\psi\}=\left[k_{r}\right] \\
\{\psi\}^{T}[C]\{\psi\}=\beta\left[k_{r}\right]
\end{gathered}
$$


Since the mode shape vectors are subject to some indeterminant scaling factor, the modal mass and stiffness are not unique values. In most cases the mode shape vectors are scaled such that the largest element has a value of one (Ewins, 2000). Mass normalization is often used as a scaling technique for normalization of the mode shape vectors, and was the technique implemented when studying mode shape correlation. Mass normalized mode shape vectors are denoted as $\{\varphi\}$ and when substituted into equations 2.11 and 2.12 the results are as follows where [I] denotes the identity matrix.

$$
\begin{gathered}
\{\varphi\}^{T}[M]\{\varphi\}=[I] \\
\{\varphi\}^{T}[K]\{\varphi\}=\left[\omega_{r}^{2}\right]
\end{gathered}
$$

The orthogonality property can also be used as a check to determine if two eigenvectors are identical. This is done be comparing the transpose of one vector with the original form of another to determine their orthogonality. The expected result from this check is shown in the equations below, where unit modal mass (UMM) normalized modes shape vectors are compared.

$$
\begin{gathered}
\{\varphi\}_{i}^{T}[M]\{\varphi\}_{j}=0 \text { for } i \neq j \\
=1 \text { for } i=j \\
\{\varphi\}_{i}^{T}[K]\{\varphi\}_{j}=0 \text { for } i \neq j \\
=\left[\omega_{r}^{2}\right]_{i} \text { for } i=j
\end{gathered}
$$

The principle of orthogonality is used to validate the mode shapes found both computationally and experimentally. The modal assurance criterion (MAC) was implemented to determine the confidence with which mode shapes could be experimentally determined as a function of number of DOF's. The equation used to compute the MAC is shown below.

$$
\operatorname{MAC}(i, j)=\frac{\left|\{\varphi\}_{i}^{T}\{\varphi\}_{j}\right|^{2}}{\left(\{\varphi\}_{i}^{T}\{\varphi\}_{i}\right)\left(\{\varphi\}_{j}^{T}\{\varphi\}_{j}\right)}
$$

The ideal result for two sets of mode shapes would be the identity matrix, however during experimental testing there are many sources of error which may contaminate the results. These 
include non-linearities in the test structure, noise in the data and inappropriate selection of measurement DOF's (Ewins, 2000). This third issue is of most interest for the data presented, as the MAC was primarily used to determine if the placement of DOF's was sufficient during testing. This was done by performing an AutoMAC computation, in which an identical set of experimental mode shape vectors are input into equation 2.18. The MAC was also useful for providing an indication of the level of correlation between the experimental and computational mode shapes; however it does not confirm correlation between two sets of eigenvectors. Ewins introduces many improved versions of the MAC, but the method used to compare two different sets of mode shape vectors was the pseudo orthogonality check (POC) (Ewins, 2000). The POC confirms orthogonality by comparing the transpose of the UMM normalized computational mode shape vectors $[\varphi]_{A}{ }^{T}$ against the UMM normalized experimental mode shape vectors $[\varphi]_{E}$. Once again, the expected result from this computation is the identity matrix, however it is expected that sources of error will be present in both the computational predictions and experimental data. It is generally accepted that diagonal terms above 0.8 and off diagonal terms below 0.2 indicate that two sets of mode shape vectors show acceptable correlation and having diagonal terms above 0.9 and off diagonal terms bellow 0.1 would indicate a strong level of correlation (Ewins, 2000).

$$
\{\varphi\}_{A}^{T}[M]\{\varphi\}_{E}=[I]
$$

Experimental mode shape vectors contain one DOF for each measurement location on the test structure. The placement of measurement locations is guided by multiple factors including preliminary checks such as the AutoMAC described earlier. Using this method ensures that each mode shape in the bandwidth of interest can be properly characterized. Test feasibility is another factor that must be considered to avoid unreasonably long testing periods which can introduce experimental error. As such, there is a practical limit to the number of degrees of freedom used during an experimental test. The number of DOF's in a computational model is determined by the size of the mesh, with 6 degrees of freedom used to represent each node in the model. As a result, 
the size of the mass matrix and computational mode shape vector are often many orders of magnitude larger than the size of the experimental mode shape vectors. This discrepancy in size must be addressed before the calculation of the POC or MAC, because both mode shape vectors as well as the mass matrix must have the same dimension to perform these calculations. That is, if the experimental mode shape vector has $\mathrm{N}$ terms corresponding to the number of DOF's, then the analytical mode shape vector must have $\mathrm{N}$ terms and the mass matrix must have size $\mathrm{N} x \mathrm{~N}$.

Reducing the analytical mode shape vector is simply a matter of selecting the nodes in the model that most closely match the experimental measurement locations, and removing the rest from the vector. The reduction of the mass matrix for the POC calculation was performed using the system equivalent reduction expansion process (SEREP) by Lam. This method and others are documented by Warwick when discussing the reduction of computational mode shape vectors and mass matrices in his work modelling the modal response of a half scale aircraft tail section subassembly. "The SEREP provides an exact means of mapping between the analytical DOFs and the experimentally active DOFs.” (Warwick, Kim, \& Mechefske, 2019)

The principle of orthogonality discussed above indicates that the response of a structure can be expressed as a linear combination each eigenvector. This principle is known as the modal expansion theorem as shown in 2.20 below, where $\{\psi\}_{R}$ is the mode shape vector for mode $\mathrm{R}$ and $q_{R}(t)$ is the modal amplitude for mode $\mathrm{R}$.

$$
\{x(t)\}=\{\psi\}_{1} q_{1}(t)+\{\psi\}_{2} q_{2}(t)+\ldots\{\psi\}_{n} q_{n}(t)
$$

As can be determined from this equation, the contribution of each mode will depend on the mode shape vector and the modal amplitude of each mode. For example, if the system is excited at or near a certain resonant frequency $\left\{\omega_{R}\right\}$, then the mode shape of the system will be dominated by the mode shape vector for mode R. Generally, for experimental modal analysis wide frequency ranges are excited, such that the overall response represents a summation of the mode shapes within the bandwidth of interest. This is achieved through selection of test 
parameters such as hammer tips for impact testing, or excitation signal generation in the case where modal shakers are used. There are some situations in which not all modes in a bandwidth are excited, and these are discussed below.

To perform a successful modal experiment, the relationship between the modal parameters of a system and the resulting FRF's must be understood. The following set of equations provide a mathematical basis which guide the placement of response measurement locations, and facilitate the understanding of FRF functions produced during EMA. Equation 2.21 forms the fundamental expression of an FRF and indicates that modal properties can be found by evaluating the response characteristics of a system (Ewins, 2000).

$$
H_{j k}(\omega)=\frac{X_{J}}{F_{K}}=\sum_{R=1}^{N} \frac{A_{R j k}}{\lambda_{R}^{2}-\omega^{2}}
$$

The numerator $\left(A_{R j k}\right)$ represents the residue of mode $\mathrm{R}$ at DOF $\mathrm{J}$ with excitation at DOF $\mathrm{K}$. It is constructed of the mode shape vector for mode $\mathrm{R}\{\varphi\}_{R}$ where $\left\{\varphi_{J}\right\}_{R}$ is the relative displacement of point $\mathrm{J}$ as a result of resonance at the $\mathrm{R}^{\text {th }}$ natural frequency. $\lambda_{R}^{2}$ is the eigenvalue for mode $\mathrm{R}$ and contains both the natural frequency and damping ratio. The residue for mode $\mathrm{R}$ can also be defined by the following equation when considering UMM normalized eigenvectors.

$$
A_{R j k}=\left\{\varphi_{J}\right\}_{R}\left\{\varphi_{K}\right\}_{R}
$$

It is this equation which carries an important implication for EMA as clearly the residue is directly dependent on the eigenvector value for both point $\mathrm{J}$ and $\mathrm{K}$. The magnitude of the FRF is therefore dependent on the input and output DOF's, so while FRF peaks will align in terms of natural frequency, their magnitude will vary. This phenomenon has a direct impact on the selection of excitation and response locations. If for example one was to place the point of excitation on a node of a certain mode $\mathrm{A}$, then $\left\{\varphi_{K}\right\}_{A}$ would be a vector with all terms equal to zero. Therefore, regardless of response measurement location no resonance peak will occur, and any response at this natural frequency will be entirely dictated by off resonance contributions 
from nearby modes. As such careful consideration must be taken when selecting the excitation location prior to a modal experiment, such that the driving point is not placed on a node of any modes. There are different methods available to predict the expected mode shapes of a structure prior to performing a modal test, and the method used for this experiment will be discussed in Chapter 4.

The residues also provide information about the overall shape of an FRF. In general, the FRF curve will represent a summation of the individual terms in the FRF series as per the modal expansion theorem. At or near each resonant peak it is found that the total FRF curve very closely matches each resonant peak, however discrepancies are found in the areas in between each peak. This phenomenon is shown in Figure 2.3 and is explained by the phase relationship between two resonant peaks in an FRF.

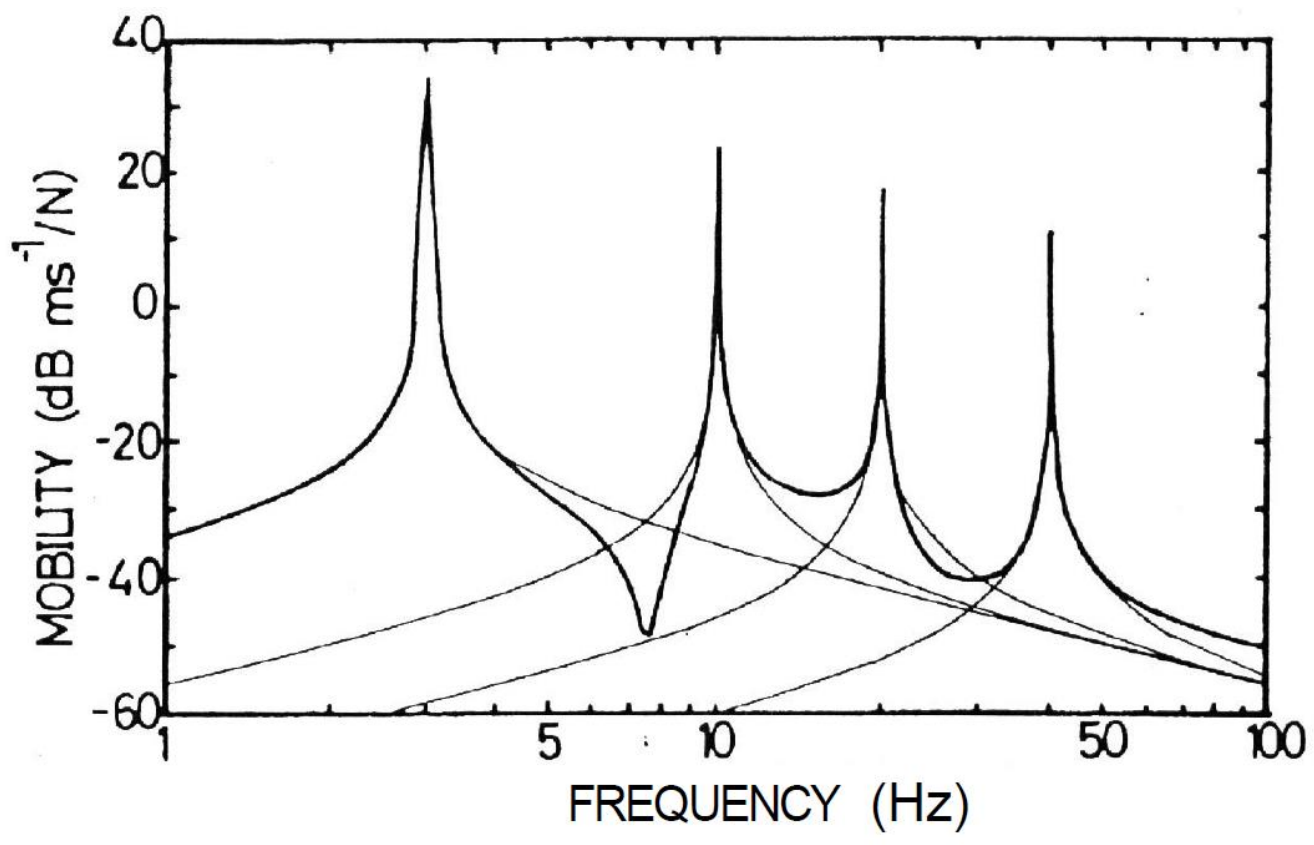

Figure 2.3 A generic mobility plot for a MDOF system. (Ewins, 2000)

The mathematical reasoning behind this phenomenon is discussed in detail by Ewins and can be reduced to some generic statements. If the residues of two consecutive modes have the same sign, then the response at some frequency between them will go close to zero and an anti- 
resonance is formed. Between two modes of opposite sign, a minima will be found (Ewins, 2000). This becomes important when considering a driving point measurement, for which the excitation and response locations are the same. In this scenario, it is expected that the residues will have the same sign throughout the frequency range of the FRF, and as such it is expected that an antiresonance will be found between every resonance peak. This is in fact the main criteria used to evaluate the experimental setup and response linearity during a driving point validity check. A typical driving point measurement is shown in Figure 2.4 below.

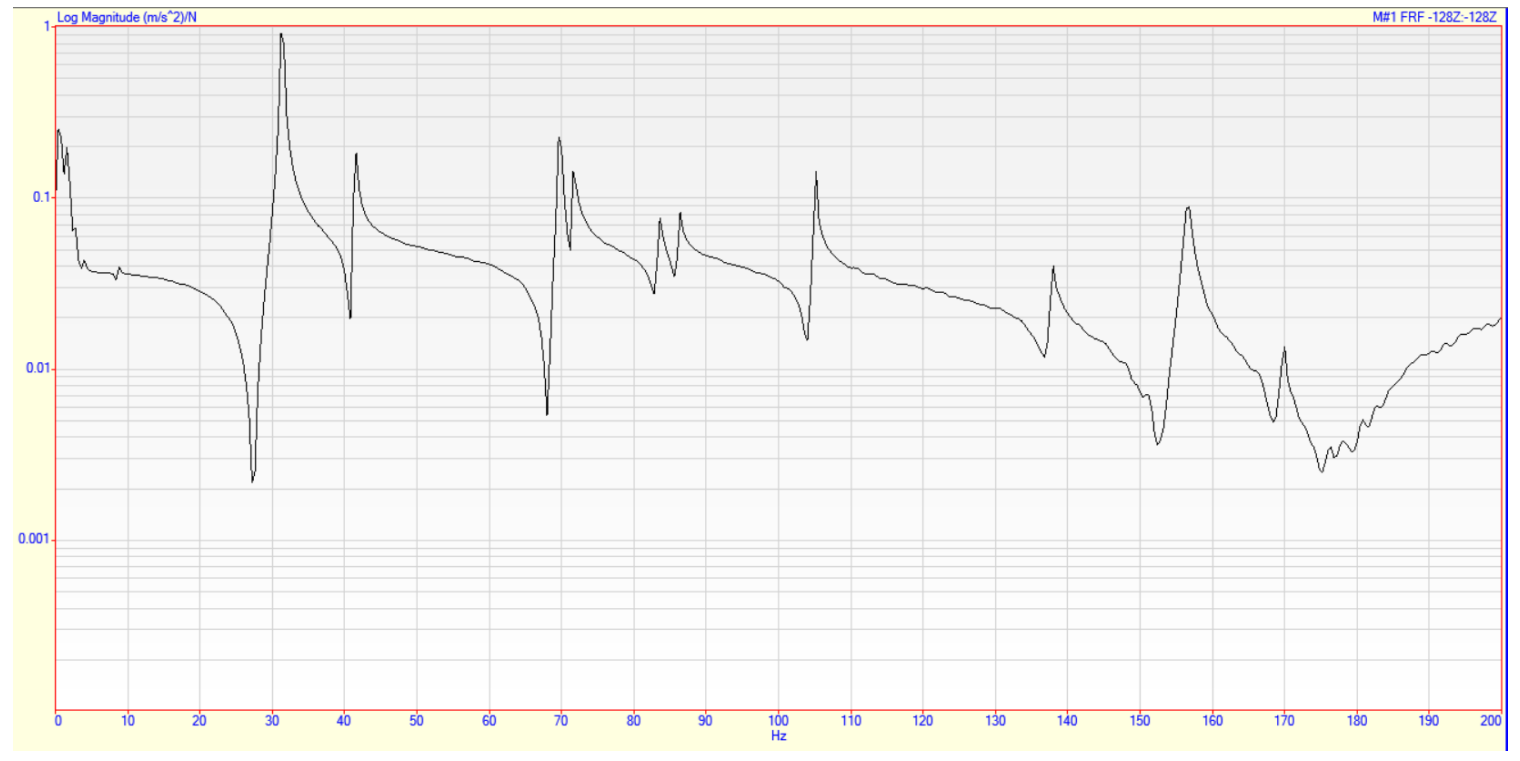

Figure 2.4:Typical driving point measurement for sub-assembly 1.

To successfully extract the modal parameters of a structure, the concept of the frequency response function matrix must be understood. This matrix is composed of a series of FRF functions each representing a potential measurement pair of excitation and response measurement locations. As such the size of the FRF matrix is determined during the planning phase of the test, where the number of DOF's required to form a complete set of eigenvalues and eigenvectors is determined. The procedure by which the arrangement of measurement locations is chosen is discussed in Chapter 4. 
One column or one row of the FRF matrix must be completed to provide enough information regarding the natural frequencies and mode shapes of the system. Each row of the FRF matrix represents a measurement during which the response measurement location is fixed, and the excitation location is roved over the entire set of DOF's. A column represents the inverse, where the excitation location is kept constant, and the response transducer is roved over the structure. The diagonal terms in the matrix represent the driving point measurement where the excitation and measurement locations are the same. For modal tests using a shaker for excitation, it is far more practical to complete a column of the FRF matrix, and this was done during the modal tests presented. The two methods stated are interchangeable due to the principle of reciprocity, which states that the corresponding rows and columns of the FRF matrix will present the same FRFs. A check to confirm the reciprocity of the structure can be performed by first exciting the structure at some DOF K, and taking a response measurement at some point $\mathrm{J}$. Then reverse this arrangement and repeat the measurement, which should produce an identical FRF to that found in the first phase of the test. This test is important to perform because according to Avitable "reciprocity is basically the reason why only one row or one column of the frequency response matrix needs to be measured.” (Avitable, 2018)

\subsection{Chapter Summary}

This chapter presents a detailed review of previous work done in the field of modal analysis, with a focus on the aerospace industry. This review spans from fundamental testing methods implemented by Gordon et al. to better understand the fatigue life of aerospace components, to a novel integration of fiber bragg grating sensors into the composite structure of a wing to detect changes in dynamic properties (Gordon, Wolfe, \& Talmadge, 1977). Additionally, a review of research performed by Chamberlain et al. was presented to explain the first step of this project prior to the research presented in this thesis (Chamberlain, 2018). Finally, the work 
completed by Warwick et al. was presented as a basis for the correlation procedures which were implemented for this research (Warwick, Kim, \& Mechefske, 2019).

In addition to a review of the literature, a summary of the relevant background theory was presented. Starting with a mathematical representation of simple SDOF dynamic system, concepts including the frequency response function and correlation methods for MDOF systems are presented. It is necessary to understand these concepts in order to complete a successful modal test, however there is an abundance of extra material which can be found in the referenced text (Avitable, 2018) (Ewins, 2000). It is now appropriate to move forward to describe the construction of both fuselage sub-assemblies in Chapter 3, and the experimental methodology used to test them in Chapter 4. 


\section{Chapter 3}

\section{Fuselage Sub-Assembly Design and Construction}

\subsection{Overview}

This chapter outlines the design and construction of the two-half scale tail section fuselage sub-assemblies which form an intermediate step between the individual components and the full tail section model. It will first provide a summary of the design process implemented on the full half scale fuselage tail section by Chamberlain. This summary includes a detailed description of the individual components of the tail section, as well as their functional purpose as part of the larger structure. Many of the individual components were constructed previously for testing by Chamberlain, and these structures and the process used to construct them are covered in detail in (Chamberlain, 2018). This chapter will focus primarily on the integration of these components into larger sub-assemblies for testing. Multiple challenges were overcome during construction using non-intuitive solutions, which should be implemented during the construction of the full tail section. These construction techniques as well as future procedural improvements will also be covered in detail.

Simplifications were made during the design and assembly of the tail section subassemblies to facilitate construction with the available resources. The other primary difference between the model and the production aircraft tail section, was the difference in scale. The tail section model was designed to be a half scale interpretation, due to the amount of laboratory testing space available. This choice, as well as the other simplifications, were made in accordance with advice provided by industry experts from the aircraft manufacturer partnered with this project. It is acknowledged that the model does not exactly represent the production aircraft, however the objective of this project is primarily to observe trends, such that design improvements could be recommended, and modelled with confidence. 
The following subsections detail the design and construction of two tail section subassemblies. Two iterations of the first sub-assembly were constructed due to an issue with the first version found during preliminary tests. This assembly was initially constructed with three skin sections that were joined only to the central frame. This lack of structural connection between skin sections had a significant effect on the dynamic response of the skin during modal tests, so a modification was required. For the second version, the mechanical connection between skin sections was improved, and so was the response of the skin during tests. The construction of the second sub-assembly proved to be a significant challenge, partly due to the scale of the structure, but largely due to a lack of knowledge regarding techniques that would prove to be invaluable for the assembly of this structure. The process of manufacturing this second subassembly is covered in detail, as the knowledge obtained from this process will help to expedite the assembly of the full tail section model. Finally, recommendations will be made for additional manufacturing improvements that will improve the construction process, and the quality of the finished product.

\subsection{Component Design Overview}

Both sub-assemblies were constructed from a set of components including frames, stringers, center bulkhead panel(s), and sections of skin. The skin sections and center panels including both engine support structures and the rear bulkhead, were cut from large $1.6 \mathrm{~mm}$ thick panels of 6061-T6 aluminum. To cut out the required shape, a waterjet was used in conjunction with a computer aided machining (CAM) software. The frame sections were first formed by rolling an initially straight section of 6061-T6 aluminum "C" channel to the desired radius. The necessary cut-outs and holes were cut using a computer numerical control (CNC) mill and the same CAM software. Finally, the stringers were cut from 6061-T6 "T" channel using the same technique used for the frame sections, with no rolling required. Specific details regarding the design process and specific manufacturing challenges for each component are included in 
subsections 3.2.1-3.2.4 below. To reference their functionality in each sub-assembly, refer to section 3.3.1-3.4, which describe the completed fuselage assemblies, and their similarities and differences with production jet fuselage sections.

\subsubsection{Skin Sections}

To create the truncated conical shape of the aircraft fuselage skin, a flat sheet must be cut into a shape analogous to the one shown in Figure 3.1, which shows a template for the entire skin section of the first sub-assembly. This shape was created using multiple functions built into SolidWorks, however these values can also be determined using a cone calculator, which can be found online. The values shown below are necessary to create a cone with a large diameter of $2204.8 \mathrm{~mm}$, a small diameter of $1952.73 \mathrm{~mm}$, and an inclination angle of 7.5 degrees. The resulting cone is shown in Figure 3.2, which also illustrates the initial design for the first subassembly skin.

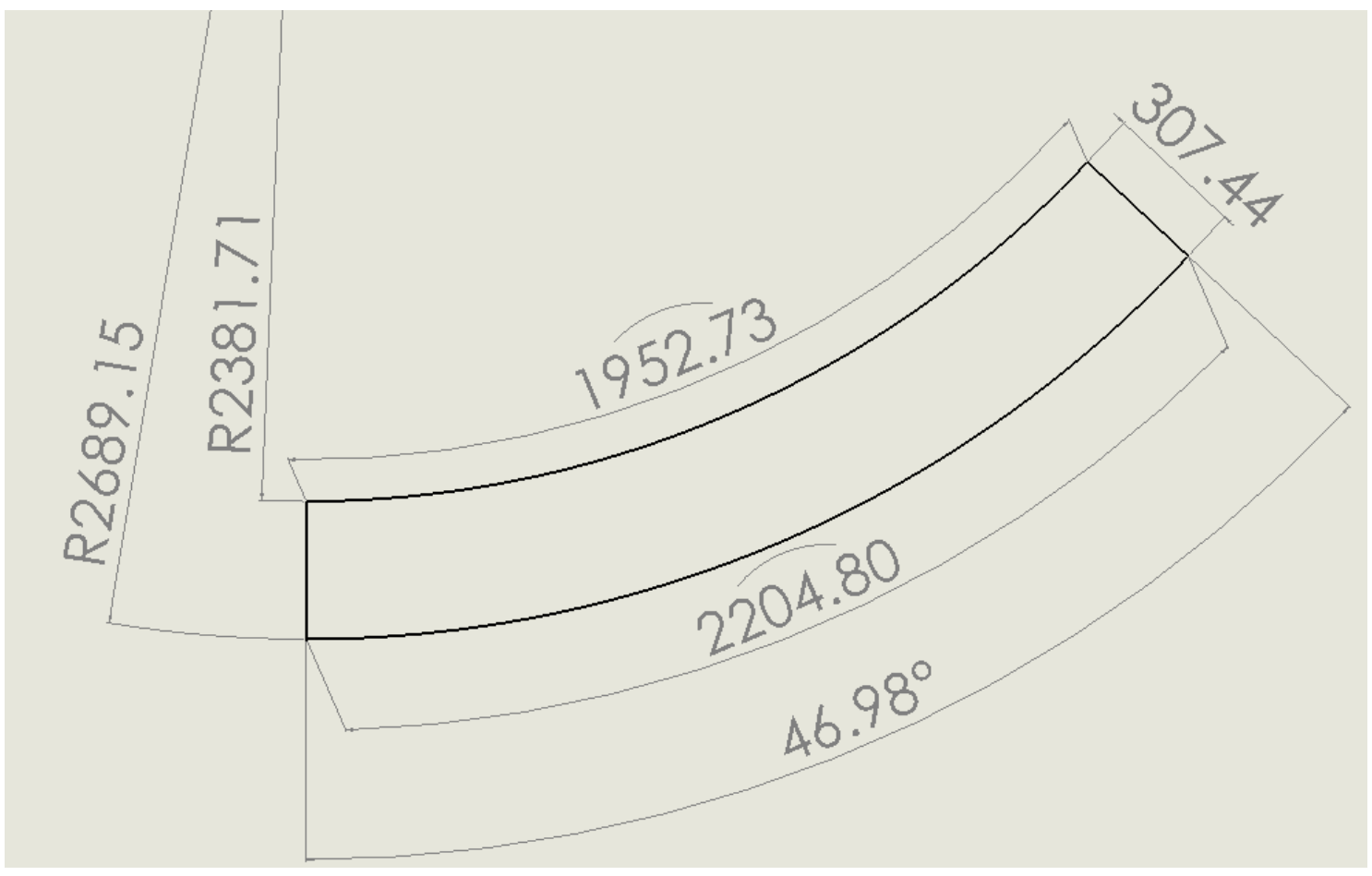

Figure 3.1: Schematic for the flat pattern of a truncated cone used for the skin of the first sub-assembly. 


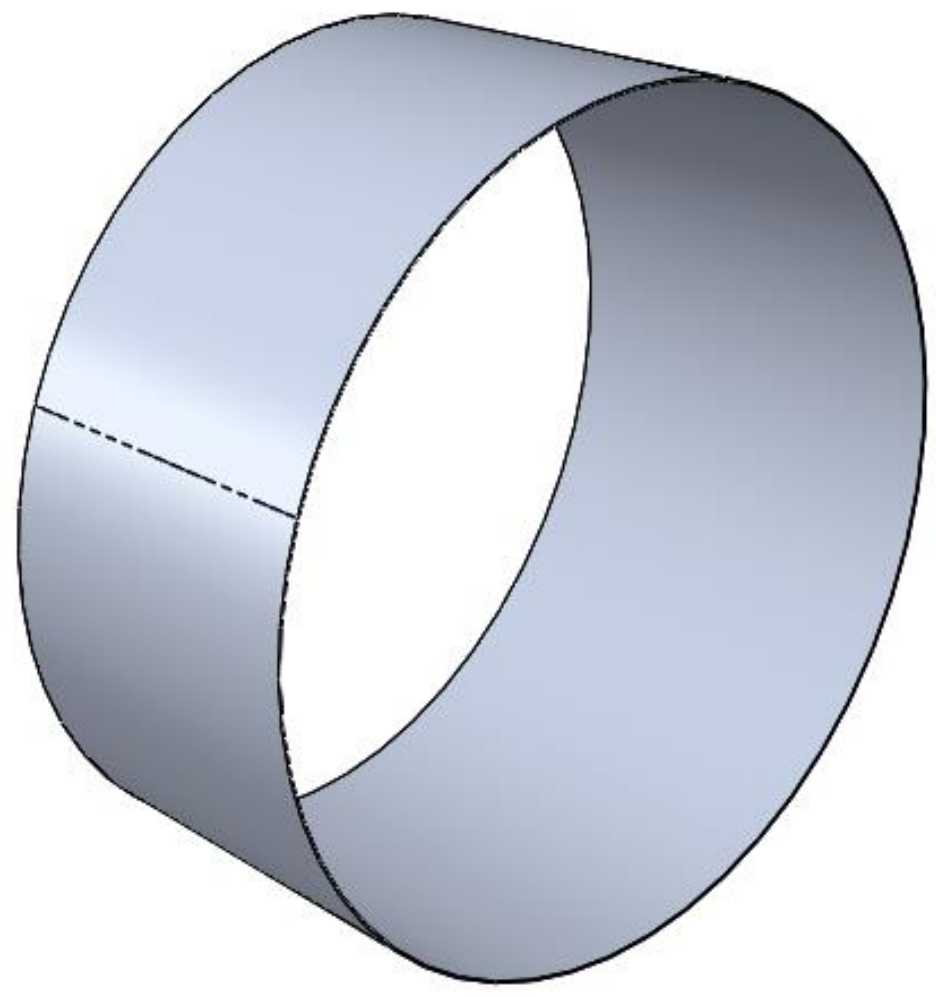

Figure 3.2: First sub-assembly skin design (first iteration).

After the necessary geometry for the skin panels was established, future iterations were created which accounted for stringer mounting, as well as available cutting space on the waterjet. To fit on the waterjet, the skin was divided into three identical sections displayed in Figures 3.3 and 3.4 which show the final design for the first subassembly skin. Mounting holes for the stringers were also included in the final design, however to save cutting time on the waterjet, these holes were only pierced then later drilled to final dimension by hand. Another intermediate iteration was created which did not have the sections of skin connect along a stringer, and this iteration is discussed in more detail in section 3.3.1. 


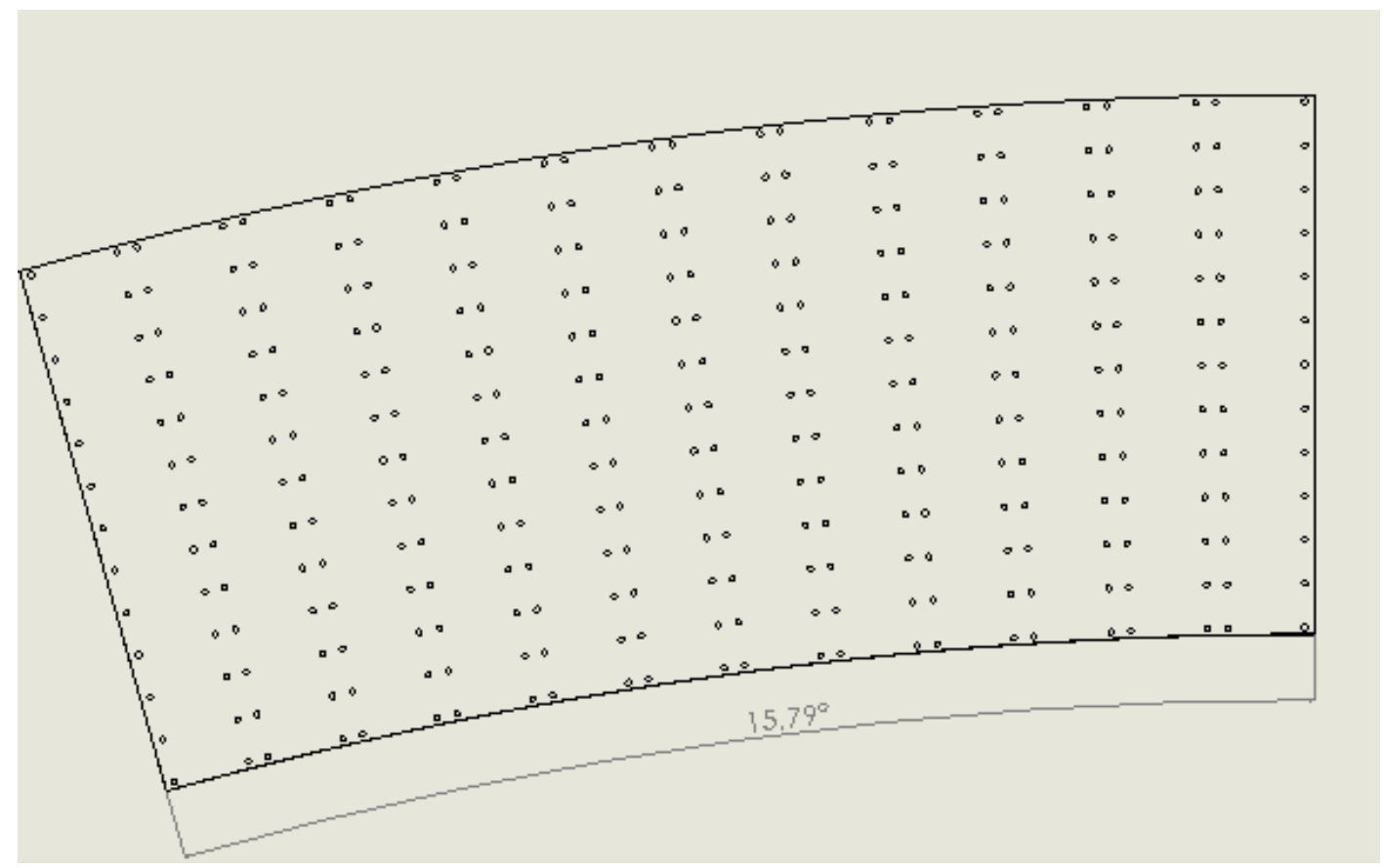

Figure 3.3: First subassembly skin flat pattern (final iteration).

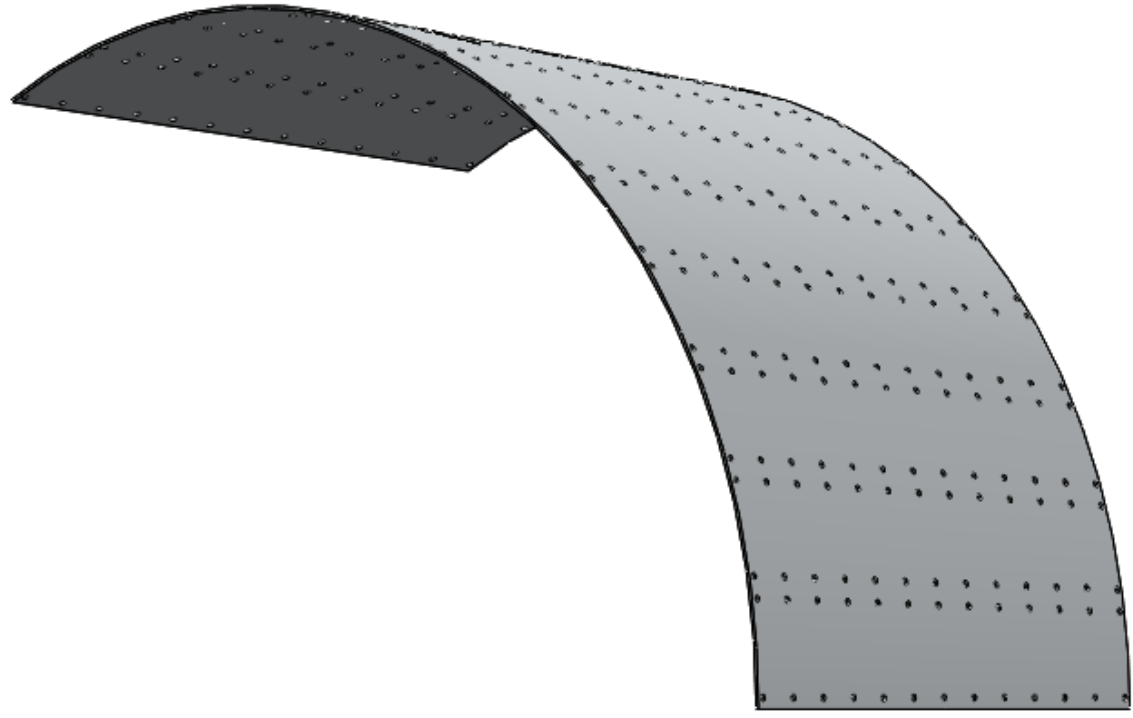

Figure 3.4: First sub-assembly skin final design.

The skin from the second sub-assembly was manufactured using an identical method to that employed on the first sub-assembly. However, this structure was far larger, and as a result the skin was divided into four sections to accommodate the limitations of the waterjet. Additional 
consideration was required when designing the cut-outs for the engine support pylons which protrude through the skin, and the design of one of these cut-outs is highlighted in Figure 3.5. In the flat pattern slots with curved edges of certain radii are required. The simplest way to design these features was to connect lines $\mathrm{A}$ and $\mathrm{B}$ at a point, then use this point as the center location for two circles which have their circumference form the two curved edges of the slot. This will ensure that when the skin section is bent during construction, the two slots form perfect rectangles to accommodate the pylons. It should also be noted that unlike sub-assembly one, these skin sections are not identical due to the placement of the pylon cut-outs, however the design process for the other sections of skin was identical.

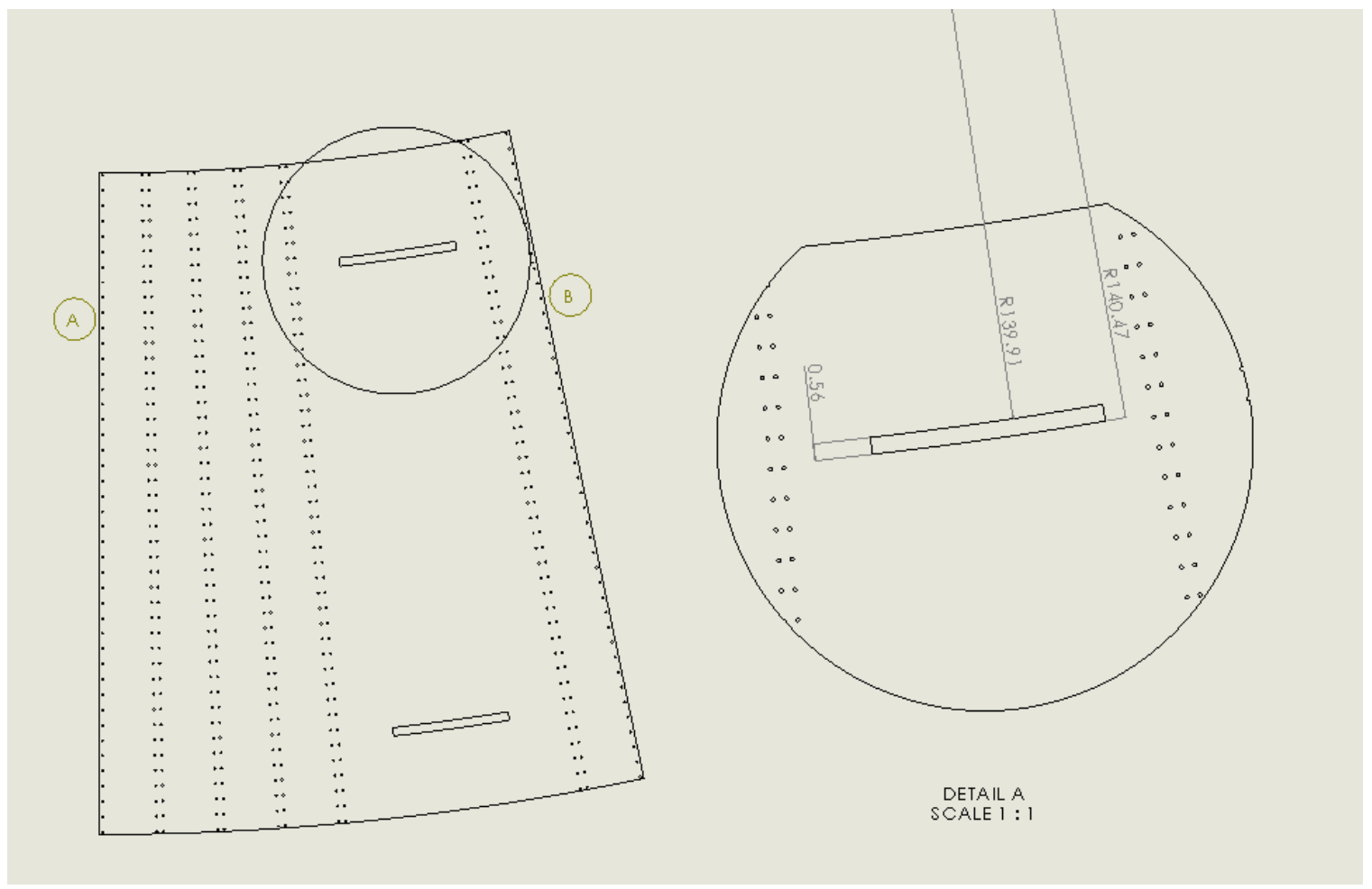

Figure 3.5: Schematic of the second sub-assembly skin, with pylon cut-out detail. 


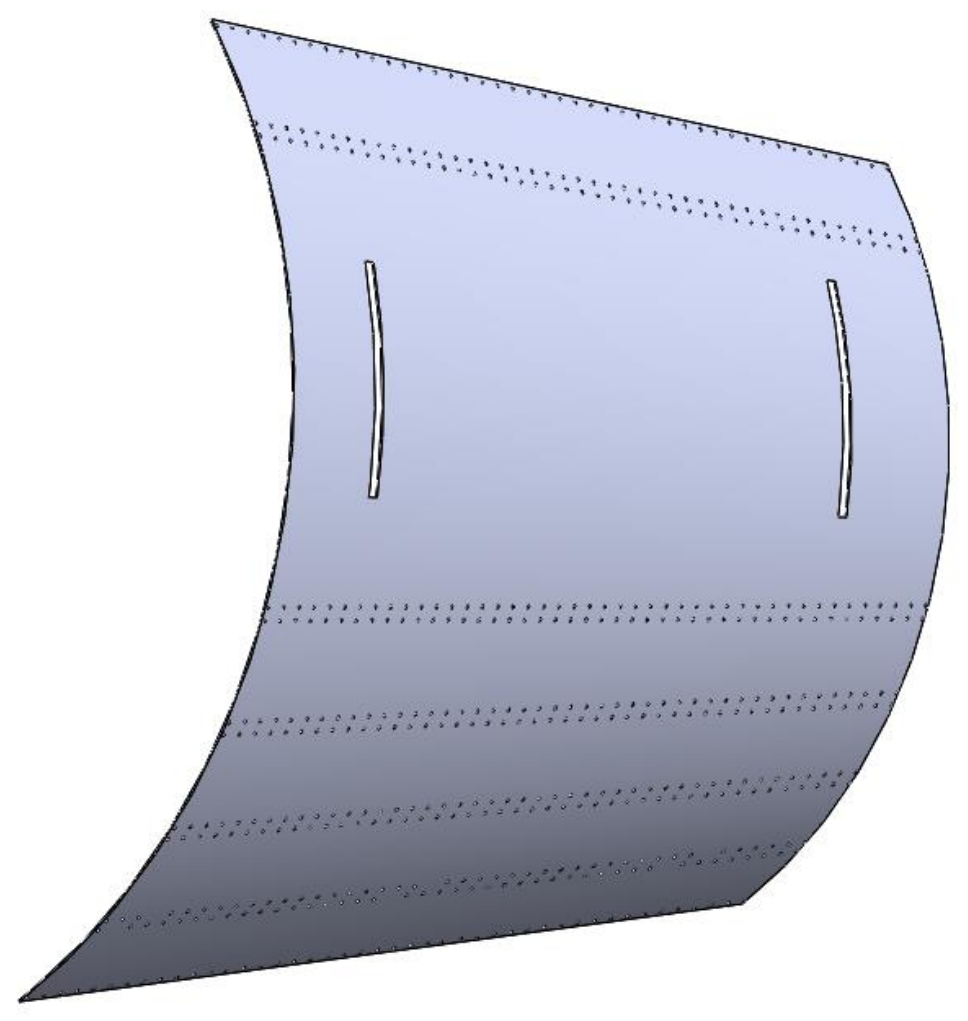

Figure 3.6: The result of bending the flat pattern in Figure 3.5 into the final conical shape.

\subsubsection{Center Panels}

Bulkhead structures are added to an aircraft fuselage to improve the structural integrity in certain areas. These structures are often found near the wings, and in the tail section of most aircraft. Therefore, to accurately represent the tail section of a business jet, these center panels must be evaluated as part of fuselage sub-assemblies. The center panels from both sub-assemblies were previously designed and manufactured by Chamberlain, but some small modifications were required to integrate these components into their respective sub-assemblies. The following subsection briefly summarizes the design and manufacturing of each component prior to integration in their respective assembly. For a more detailed description regarding the design process of these components refer to (Chamberlain, 2018).

The first sub-assembly contained one central panel representative of the aircraft's aft bulkhead. This panel forms the final structural member at the back of the plane; not to be 
confused with the pressurized bulkhead which separates the passenger cabin and the unpressurized tail section. The aft bulkhead was cut from a sheet of $1.6 \mathrm{~mm}$ thick aluminum using an identical method to that used for the skin panels. This bulkhead includes the necessary holes for riveting to its corresponding frame, and semicircular cut-outs which are necessary for clearance with the stringers. The final design of the aft bulkhead is shown in Figure 3.7.

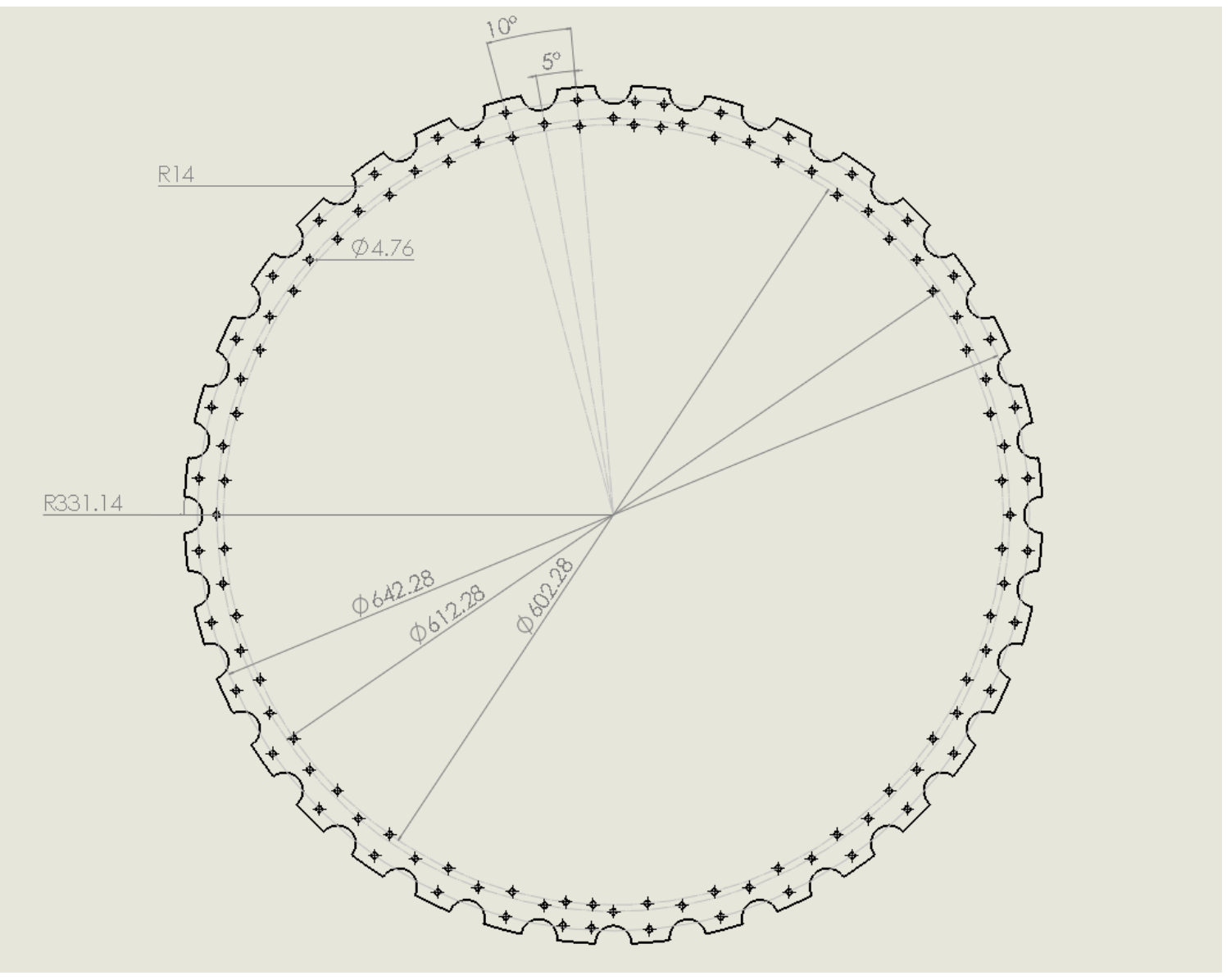

Figure 3.7: Schematic of the first sub-assembly aft bulkhead.

The second sub-assembly forms the section of fuselage tail responsible for supporting both jet engines. As such, both the front and rear engine support frames (ESFs) are included in this assembly. The engine support frames are panels which provide an improved structural connection between the engine pylons and the fuselage, by distributing the engine loads around their corresponding fuselage frame. As with the aft bulkhead, holes are required to rivet each ESF 
to its corresponding frame and cut-outs are required for stringer clearance. In addition, holes were required to facilitate the bolted connection with the engine pylons, and crawl space cut-outs were added to better represent the components present on the full-scale aircraft. These semicircular cutouts are made in the lower half of both ESFs to provide access to the rear section of the plane.

The final design for both ESFs including their corresponding pylons are presented in Figures 3.8 and 3.9.

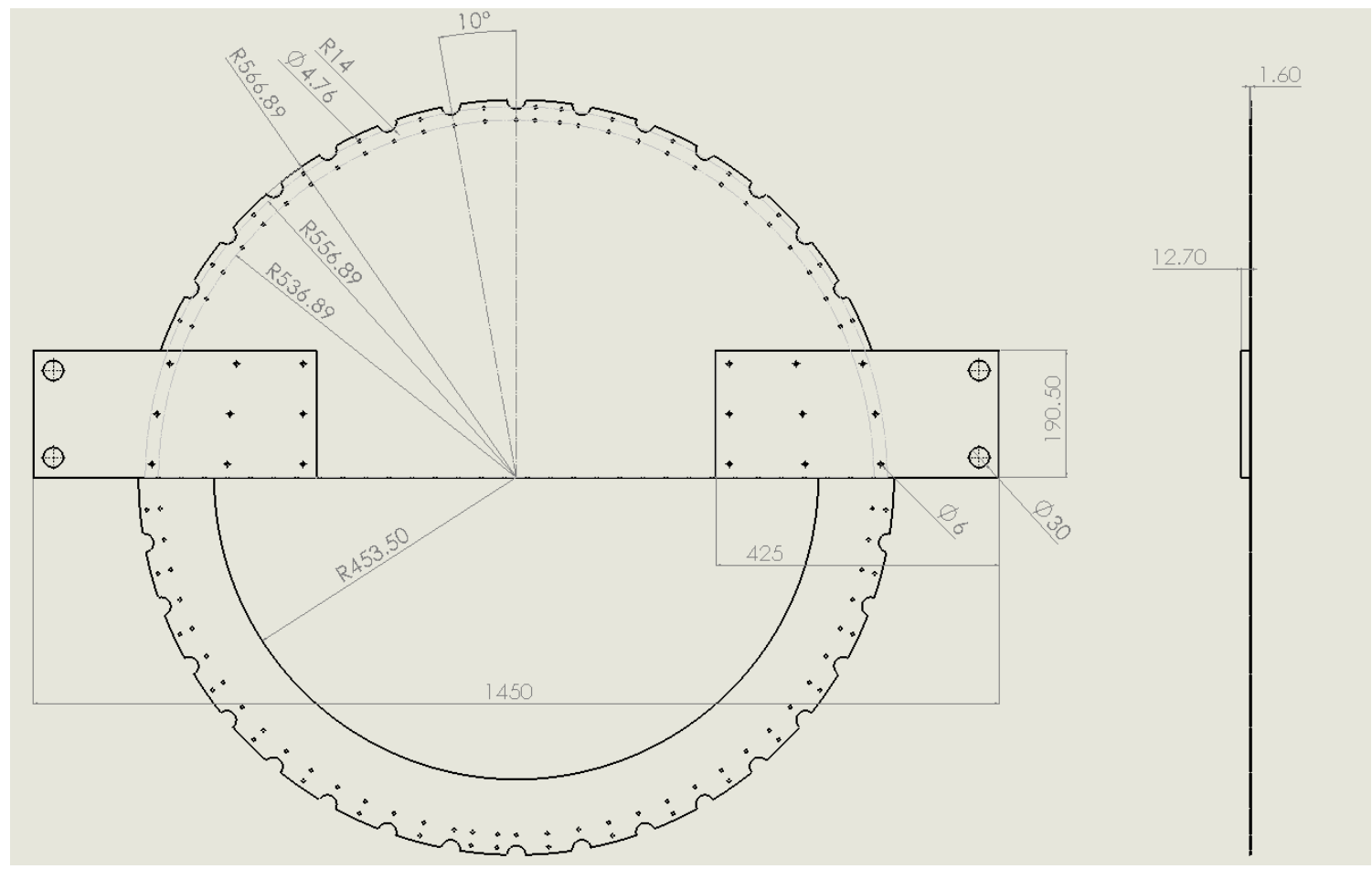

Figure 3.8: Final schematic for the front engine support frame (FESF) including engine pylons. 


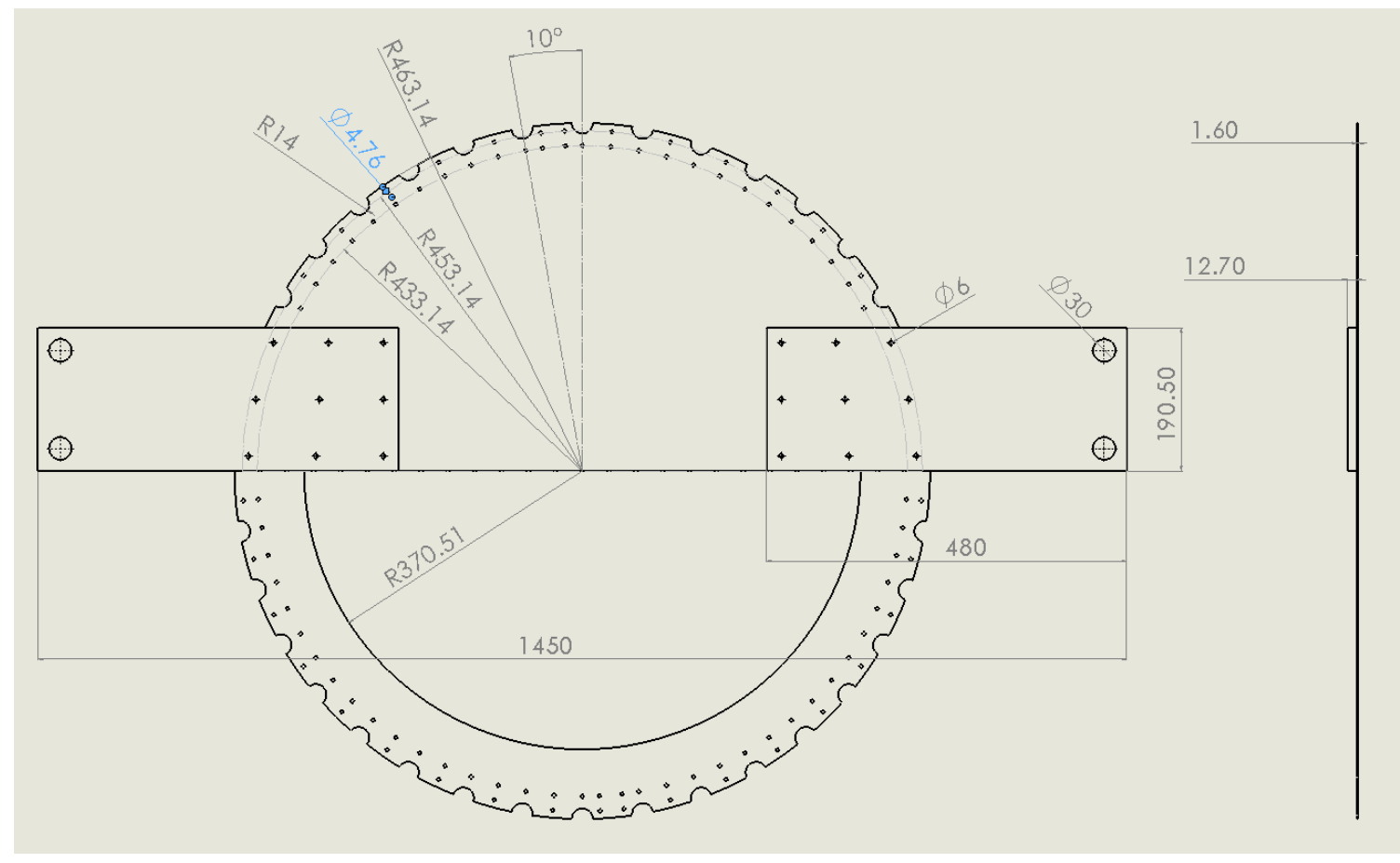

Figure 3.9:Final schematic for the rear engine support frame (RESF) including engine pylons.

\subsubsection{Frame Sections}

Aircraft frames form essential structural members within the fuselage of aircraft. They add rigidity to the skin of the aircraft and provide structurally sound mounting locations for components including the jet engines, wings, and bulkheads. On operational aircraft, geometry of the frames varies depending on the loading condition imposed at their location in the fuselage. This is done primarily to optimize the structural characteristics of the plane relative to other factors such as weight. For simplicity though, the half scale frames constructed for both subassemblies were of constant cross section. This allowed the frames to be rolled using one set of dies reducing cost significantly, and this simplification was verified through consultation with industry partners. The frames were rolled from 6061-T6 aluminum "C-Channel" to the required radius, and during this process an inclination angle of $7.5^{\circ}$ was imposed on the outer face to accommodate the conical shape of the fuselage tail section. The final design for the frame cross section is shown in Figure 3.10. 


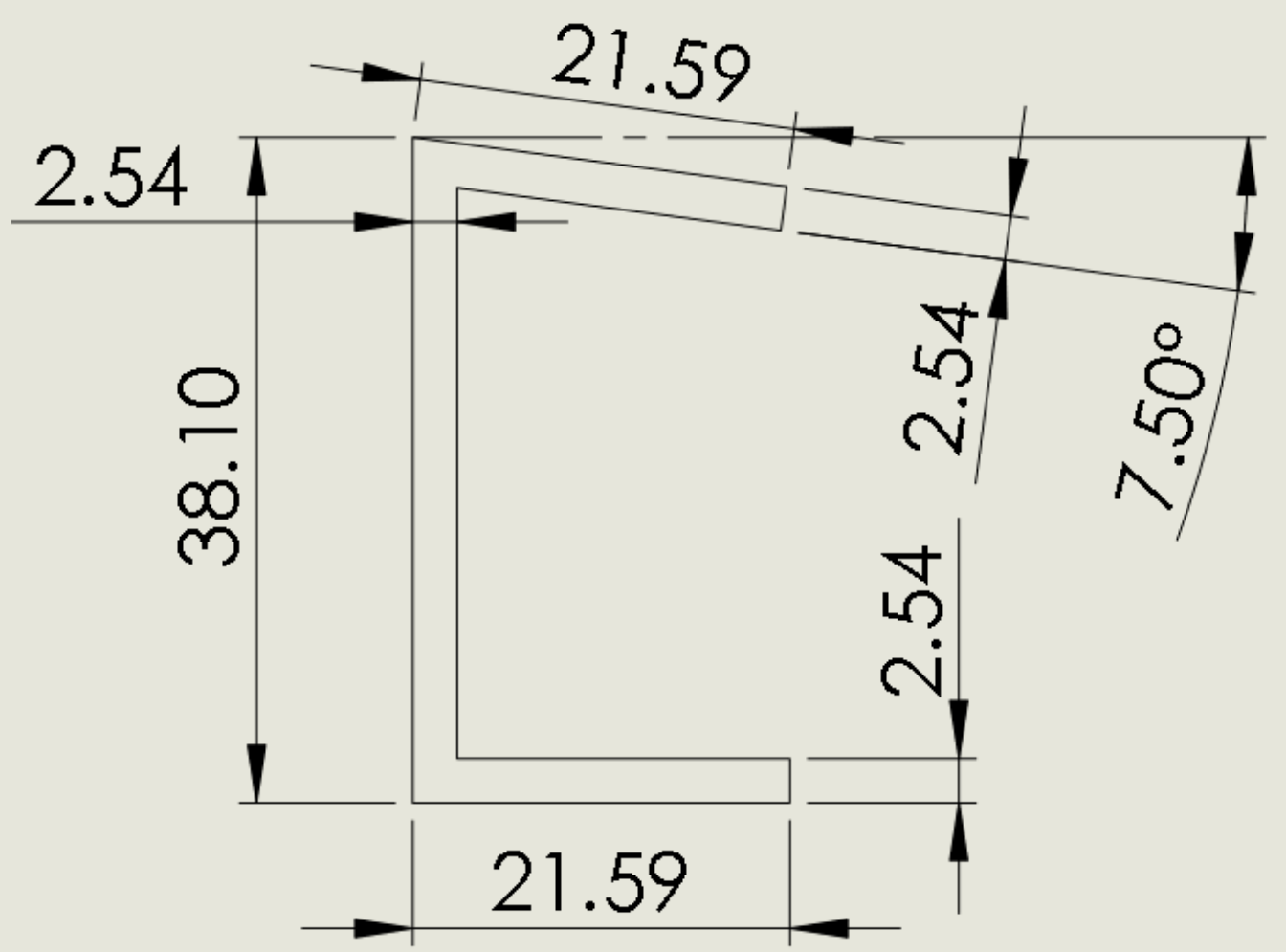

Figure 3.10: Universal cross section schematic used for all frame sections from both subassemblies.

The first step in creating each frame section is to transform the originally straight " $\mathrm{C}$ Channel" into a curved section of the required diameter, and by using custom dies during this cold rolling process the $7.5^{0}$ inclination angle can also be formed. Due to manufacturing restrictions, each fuselage frame was formed out of two identical $180^{\circ}$ sections, as it was impossible to create a complete ring using this technique. After being formed to the necessary shape, the stringer clearance cut-outs and bulkhead mounting holes were cut using a CNC mill to match up with the corresponding bulkhead panels. A schematic for a frame section from subassembly 1 (FS-1066) is shown in Figure 3.11. The frame sections for sub-assembly 2 shared the same pattern for rivet holes and stringer clearance cut-outs which was expanded to fit their larger radius. 


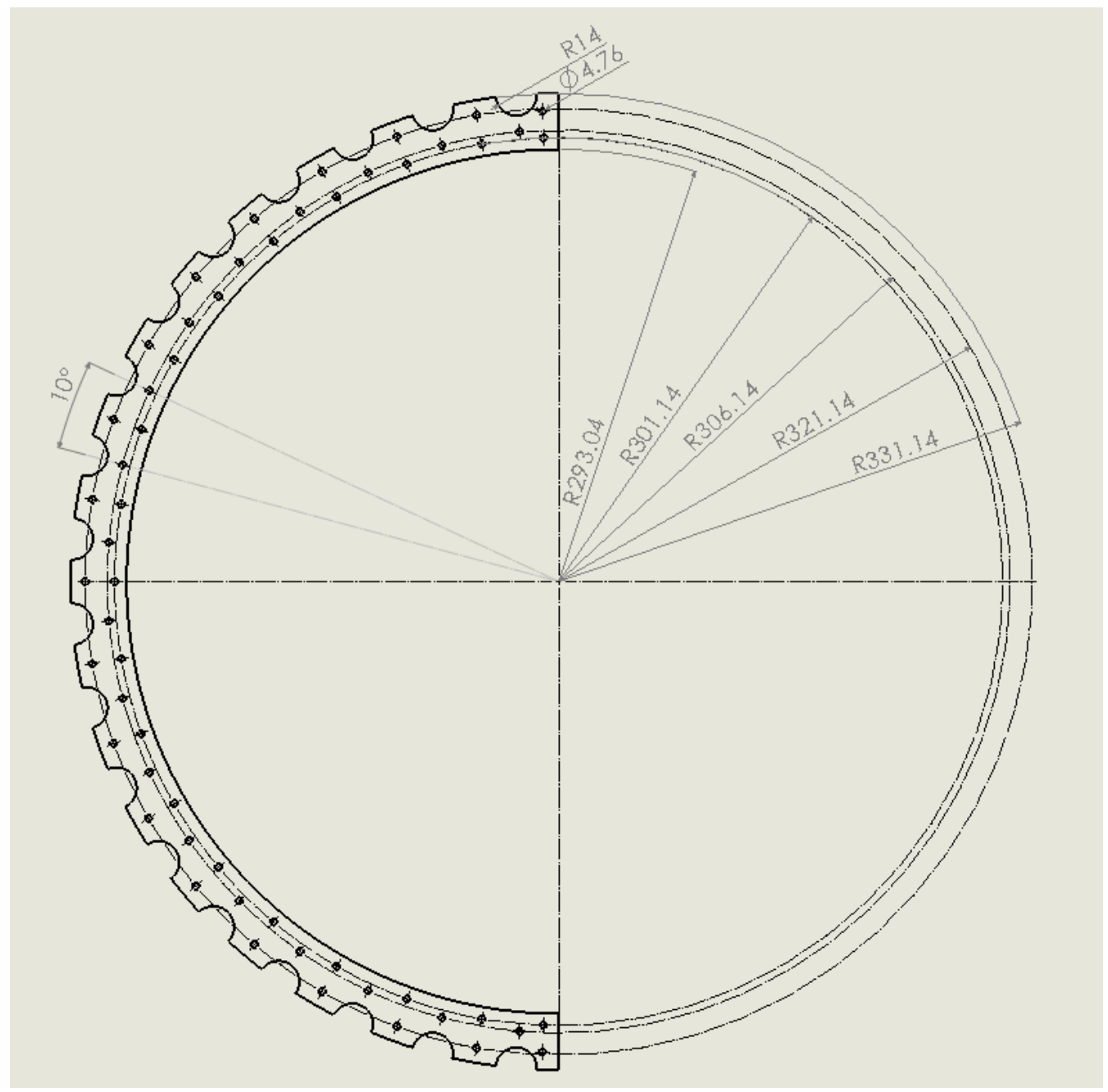

Figure 3.11: Frame section 1066 from sub-assembly 1.

\subsubsection{Stringers}

Stringers in aircraft are implemented to improve the longitudinal rigidity of the fuselage structure. Much like the fuselage frames, there is a range of different geometries used in aircraft dependent on load conditions, available clearance, and other factors. For both sub-assemblies, one uniform cross section was selected to expedite manufacturing. As with the fuselage frame sections, this cross section was inspired by structures on full scale aircraft and verified through consultation with industry partners. The choice of cross section was also partially motivated by what aluminum extrusion was commercially available, and because this "T-Channel" was mass produced externally, costs and manufacturing time were reduced. Holes were drilled in the 
stringers to facilitate mounting to their respective skin sections. The spacing of these holes was chosen based on two factors; the general spacing used in aviation, and what was deemed feasible with the available resources. Ideally, the spacing between rivets for the half scale models would be half of what is found on full scale aircraft; however this would result in an unreasonable number of rivets for either sub-assembly. After consulting with industry experts, the space between rivets was approximately doubled to expedite the manufacturing process. The final design for a stringer from the first sub-assembly is shown in Figure 3.12. The stringers for the second sub-assembly were $1112.3 \mathrm{~mm}$ long with a hole spacing of $25.7 \mathrm{~mm}$.

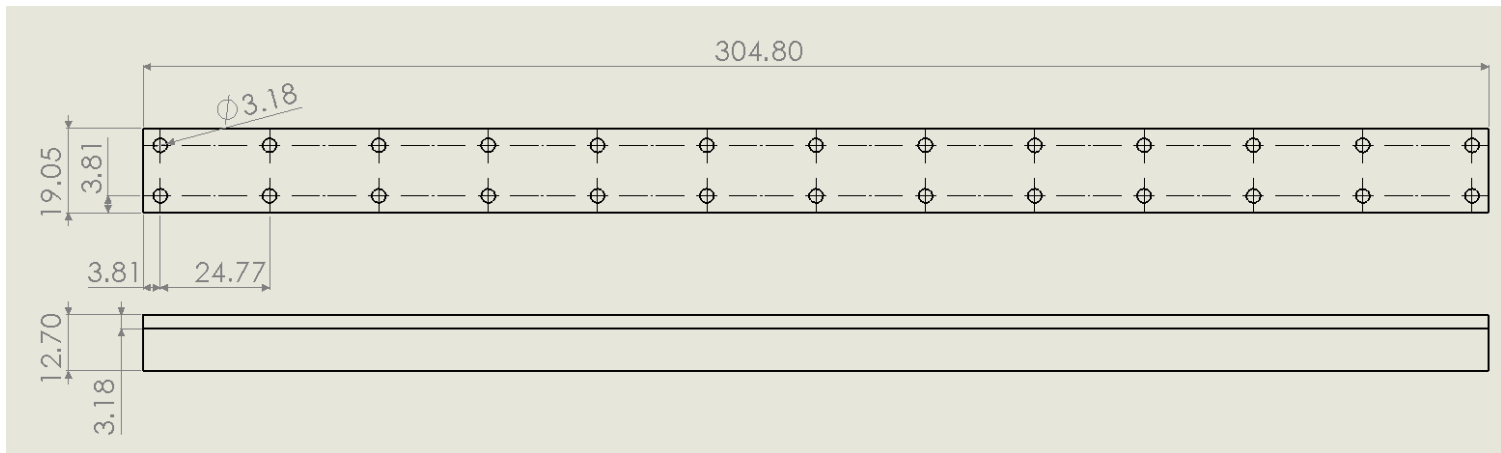

Figure 3.12: The schematic of a stringer from sub-assembly 1.

\subsection{Sub-assembly Design and Construction}

\subsubsection{Sub-assembly 1 Version 1}

The first sub-assembly was used to form a general testing methodology suitable for testing aircraft fuselage structures. It was necessary that this methodology was applicable to evaluate the modal properties of major components within different fuselage sections. This first structure would also be used to verify computational modelling techniques through the comparison of natural frequencies and mode shapes. As such, it was important that this first subassembly was made up of a central bulkhead structure as well as a section of skin and stringers. This provided a complete set of results which were used to evaluate the testing methodology and correlation with the computational predictions. Additionally, due to the trial and error approach 
used to form certain aspects of the methodology, a small section of the fuselage was desired to expedite the process. For these reasons, the aft most section of the half scale tail section model was selected for preliminary experimentation. The first sub-assembly's place within the full tail section model is highlighted in Figures 3.13 and 3.14 below.

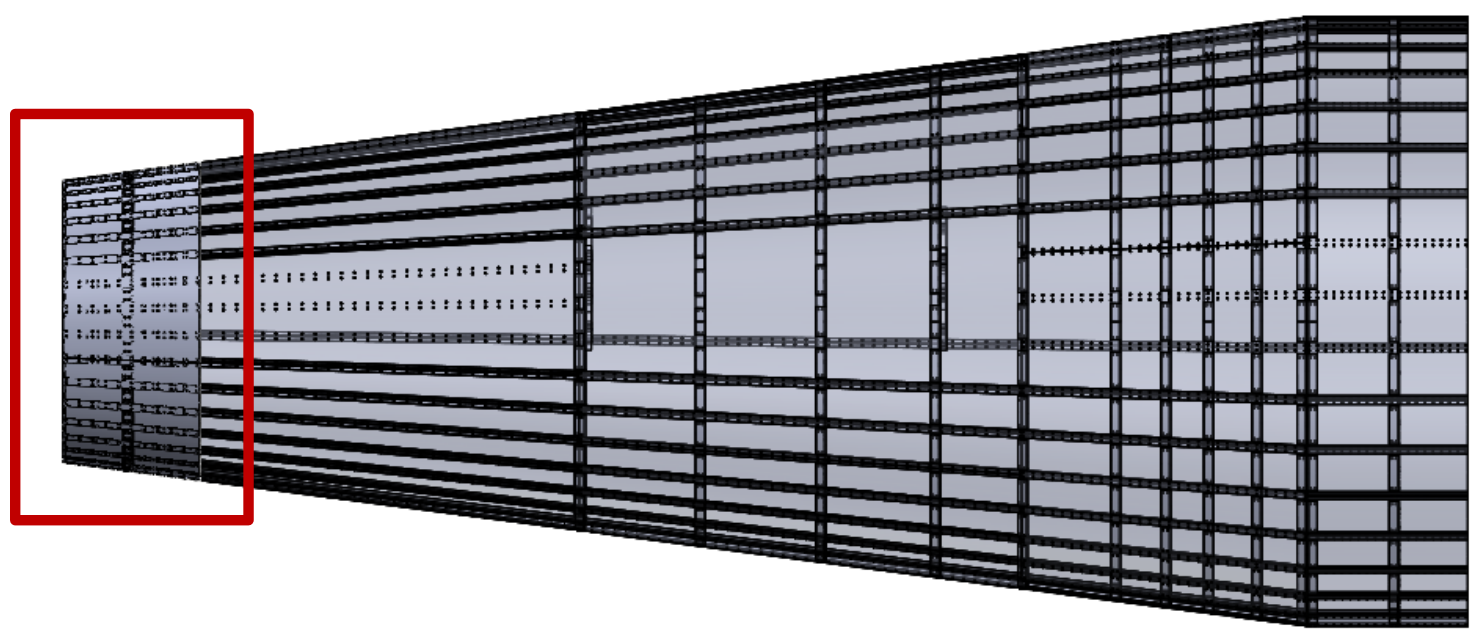

Figure 3.13: A side view of the full tail section showing where sub-assembly 1 originates.

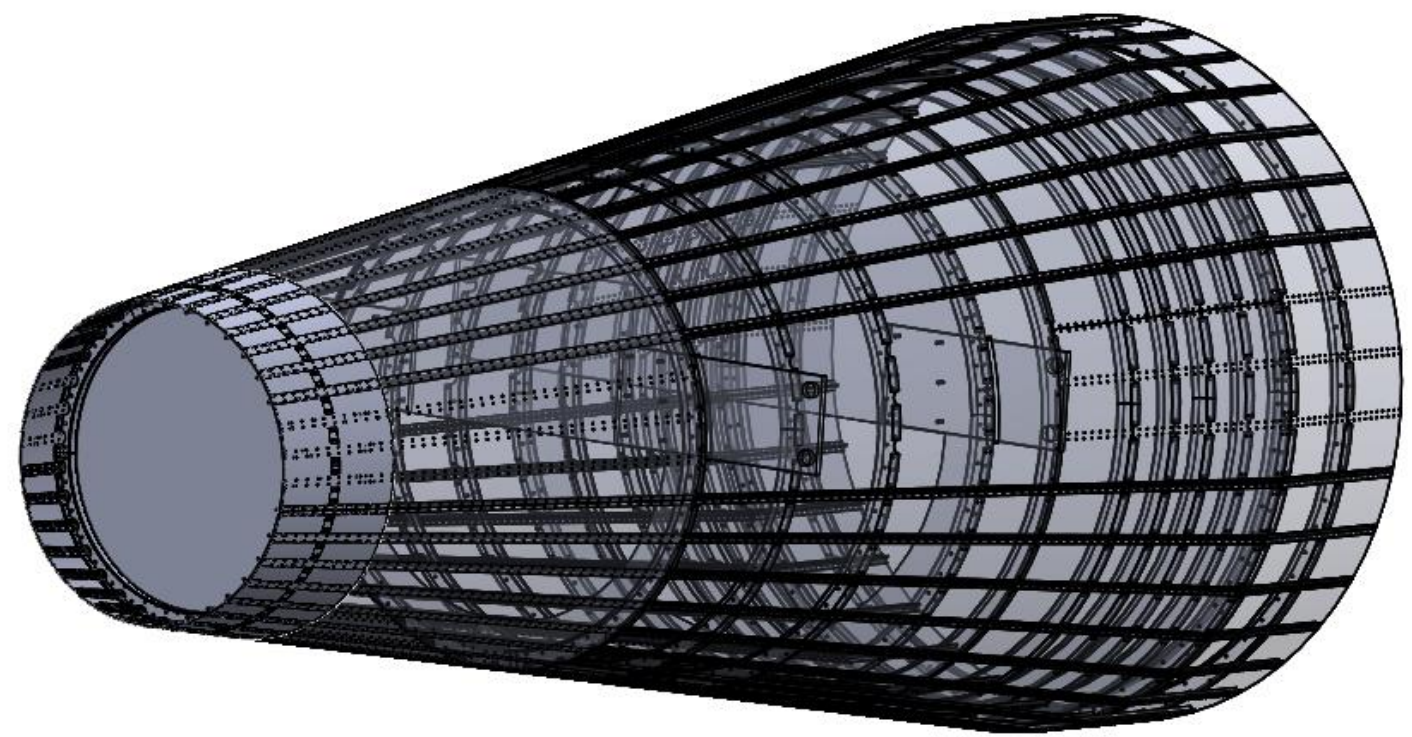

Figure 3.14: Isometric view of the full tail section showing where sub-assembly 1 originates. 
The first sub-assembly consists of the aft bulkhead, its corresponding frame, and three one-foot long sections of skin and stringers. To fasten these components together, rivets were used in accordance with general aviation practices. To reduce drag, flush rivets are the primary fasteners used on production aircraft, however the installation of these rivets is time consuming. Using blind rivets allows for quicker assembly, resulting in a more feasible project timeline. The aerodynamic properties of each sub-assembly are not of interest, and after consultation with industry experts, any structural differences between blind and flush rivets were found to be outside the scope of this project. Therefore, blind "pop" rivets of two different sizes were used to fasten these components together. To hold the aft bulkhead to the fuselage frame (FS 1066), $3 / 16$ " rivets were used, and to fasten the stringers to the skin, $1 / 8$ " rivets were chosen. The completed assembly is shown in Figure 3.15 and Figure 3.16, and Figure 3.16 highlights an issue with this version found during initial modal tests. Each skin section was connected to the central frame, but the panels were not connected to each other, and this lack of mechanical connection resulted in poor energy distribution around the structure during modal tests. Additionally, the results from this test were not representative of the modal characteristics of general aircraft assemblies which have strong mechanical connections between each section of skin. As such a revision to the design of the skin panels was made, such that they connected along a stringer. This design formed the second iteration of the first sub-assembly which is described in section 3.3.2. 


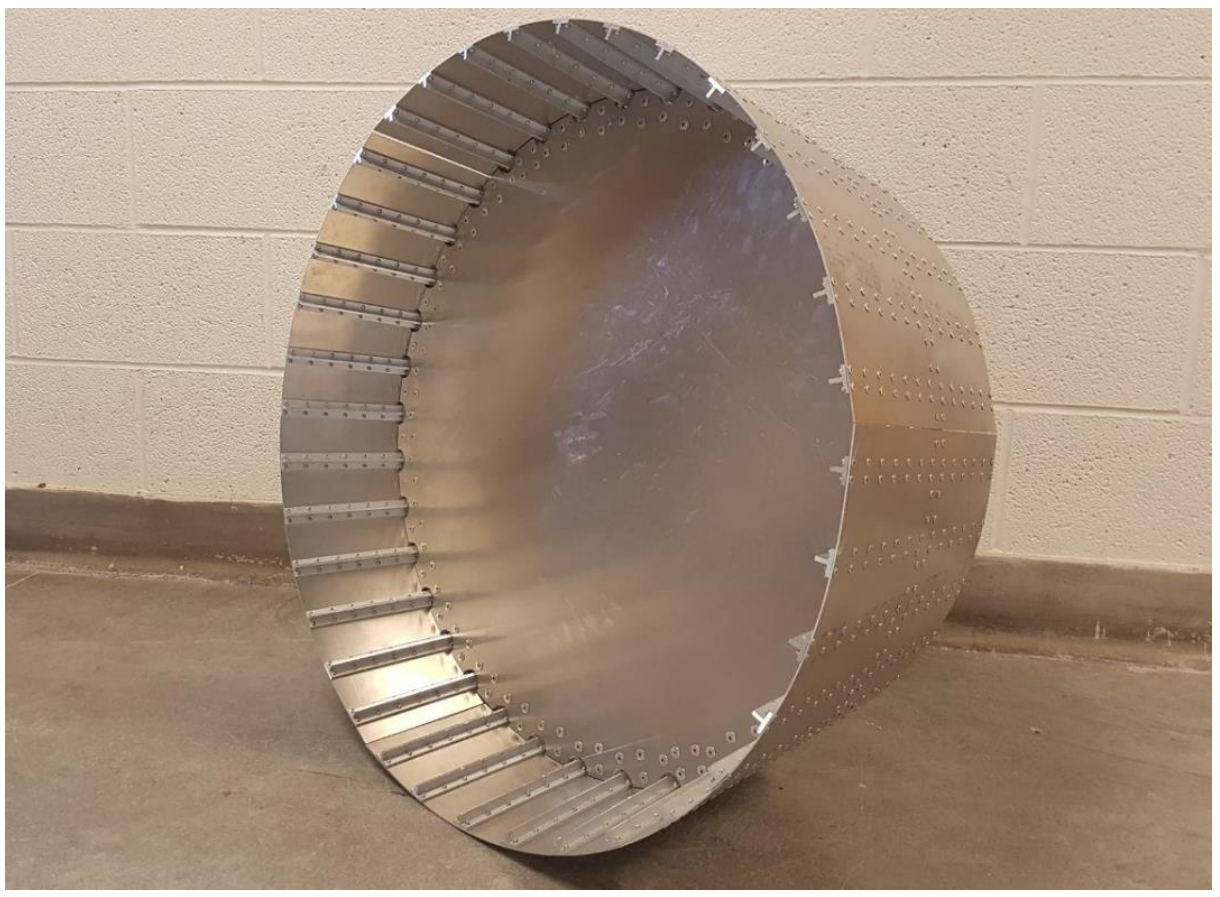

Figure 3.15: An image showing the completed first version of sub-assembly 1.

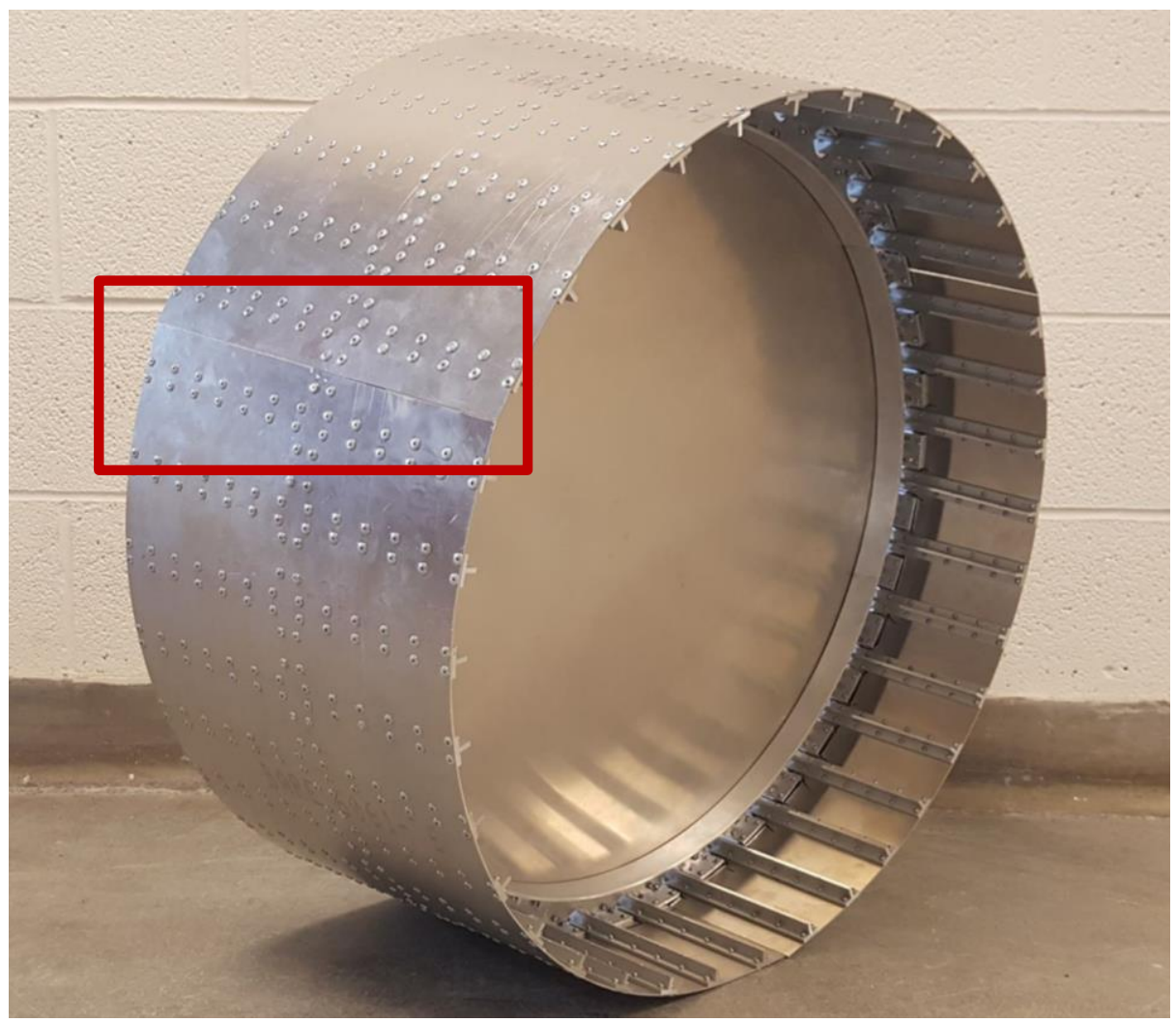

Figure 3.16: An image highlighting the poor mechanical connection between sections of skin on the first version of the first subassembly. 


\subsubsection{Sub-assembly 1 Version 2}

The second version of the first sub-assembly used the same frame sections, bulkhead and stringers as the first iteration, and three new sections of skin. These sections of skin were designed to facilitate connection between panels along a stringer as highlighted in Figure 3.17. This modification greatly improved the structural integrity of the skin, and while not a perfect representation of the lap joints or other methods employed on production aircraft, it provided a reasonable representation of what is found in industry. The eye bolts in Figure 3.17 were used to suspend the assembly for modal tests. More details regarding the boundary conditions used can be found in Chapter 4.

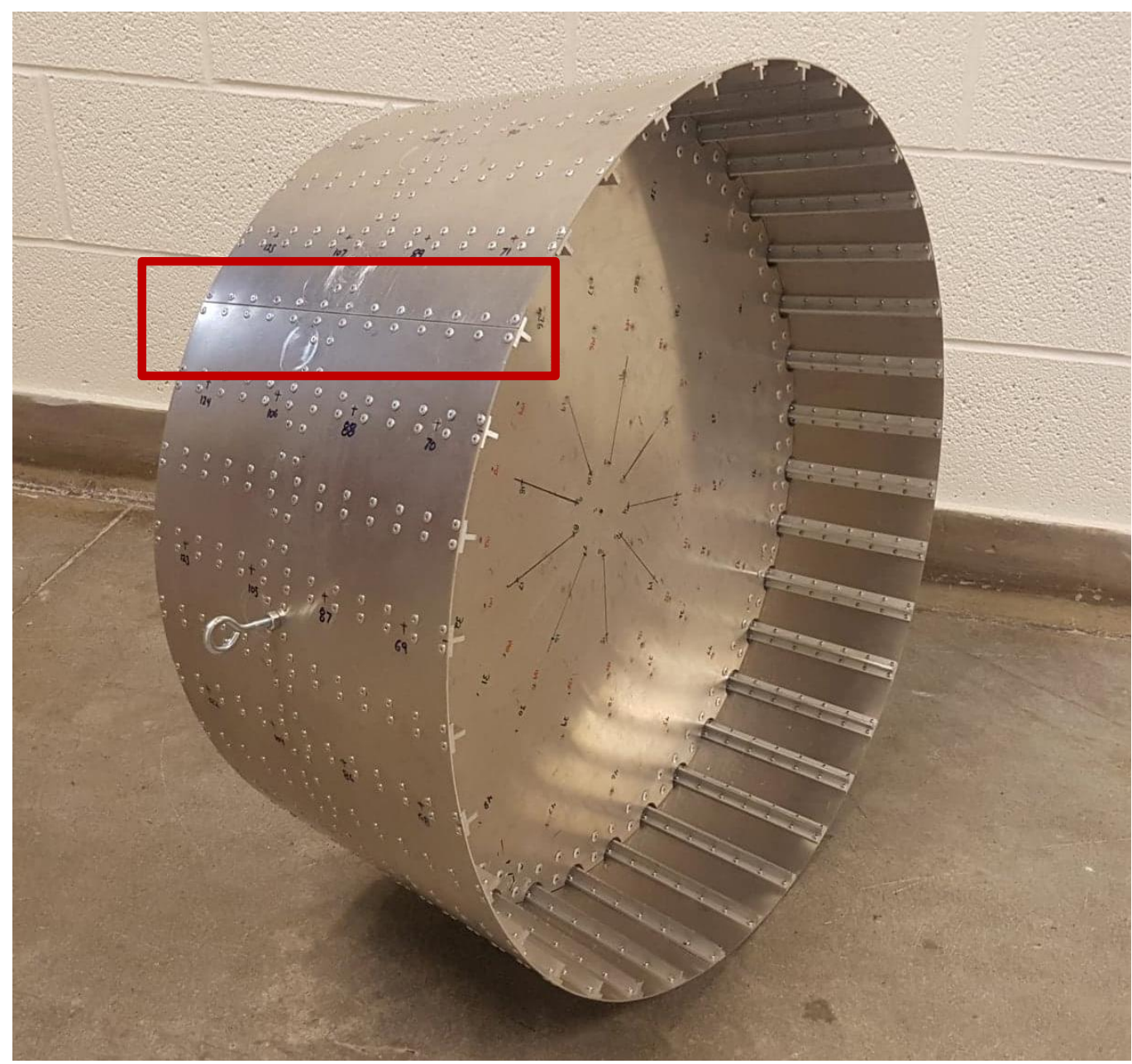

Figure 3.17:An image highlighting the improvement in mechanical connection between sections of skin on the first version of the first subassembly. 


\subsection{Sub-assembly 2}

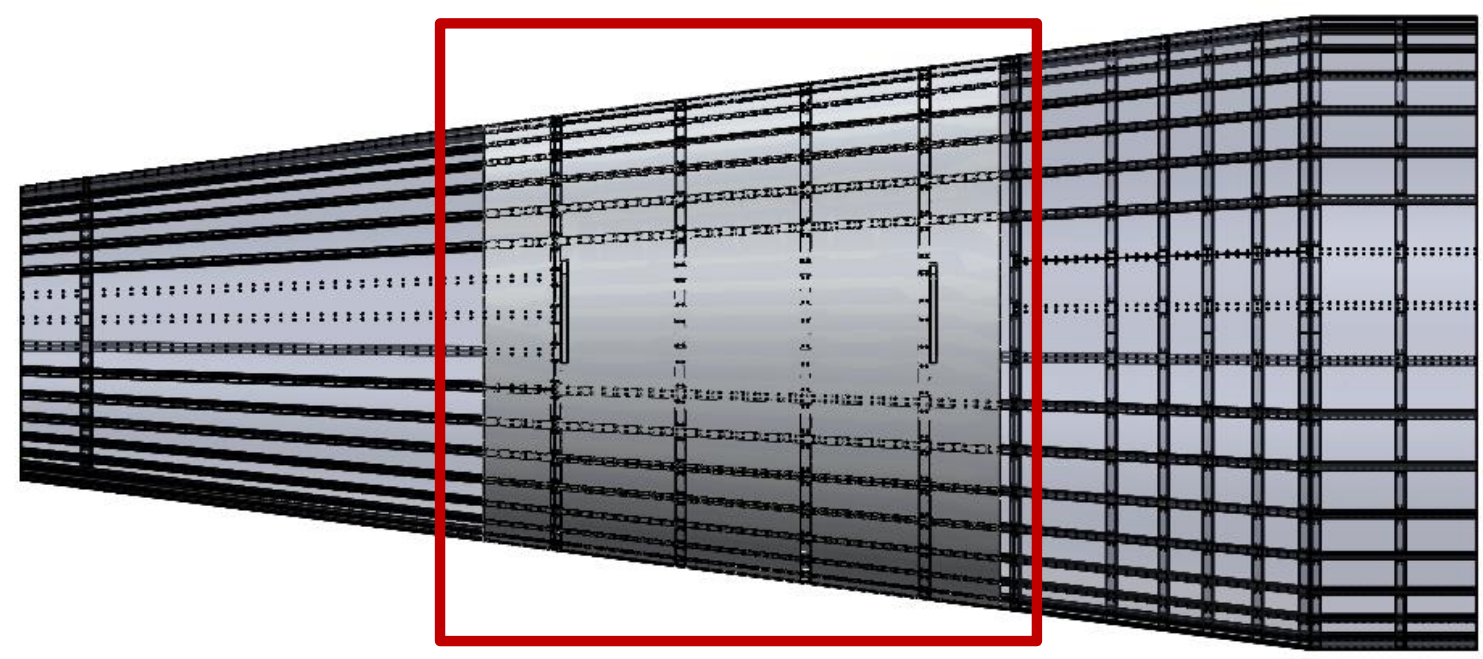

Figure 3.18: A side view of the full tail section showing where sub-assembly 2 originates

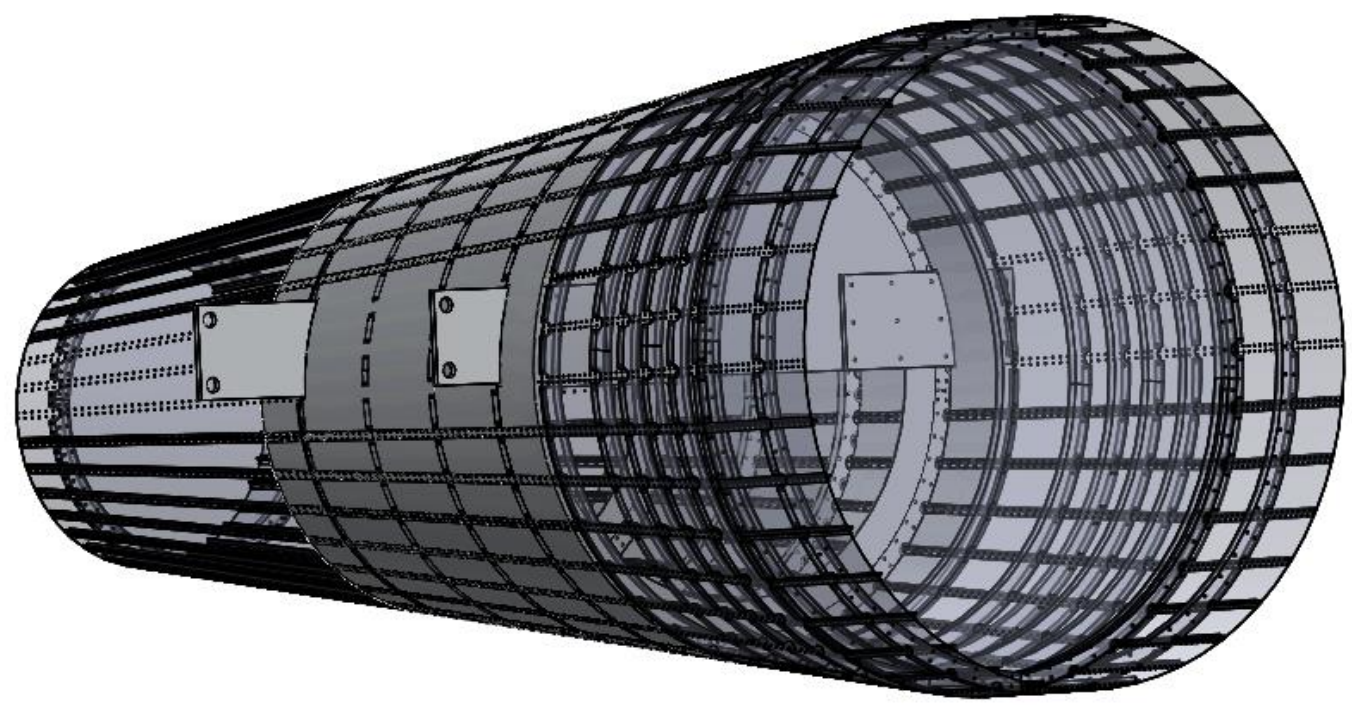

Figure 3.19: Isometric view of the full tail section showing where sub-assembly 2 originates.

Sub-assembly two models the section of fuselage responsible for supporting both jet engines. Previously, the modal properties of both ESF's were extracted by Chamberlain, and this structure was used to evaluate changes in modal properties of the ESF's as part of a fuselage structure (Chamberlain, 2018). Additionally, the results from testing this structure will serve to validate computational models and/or guide model updating where necessary. 
This sub-assembly includes the front and rear engine support frame panels, and four structural frames; two of which facilitate the connection between the skin and engine support frames. Four skin panels were required to wrap around the structure, and these were connected along stringers to form a sound structural connection. The engine pylons are bolted directly to the engine support frames and protrude through the fuselage skin. On production aircraft, fastening the pylons in a similar manner distributes the loads generated by each engine around the entire fuselage. To support the significant loads produced by the turbines in flight, significant resources are devoted to developing these components, however, to expedite the manufacturing process with the available resources, a simpler design was selected. All design simplifications were made in consultation with industry partners, and because this project was directed at finding general trends of dynamic response in fuselage structures, more detailed individual component design was deemed outside the scope of this research. Figures 3.20, 3.21 and 3.22 show the second subassembly throughout the construction process and provide a look at the internal construction of the sub-assembly. 


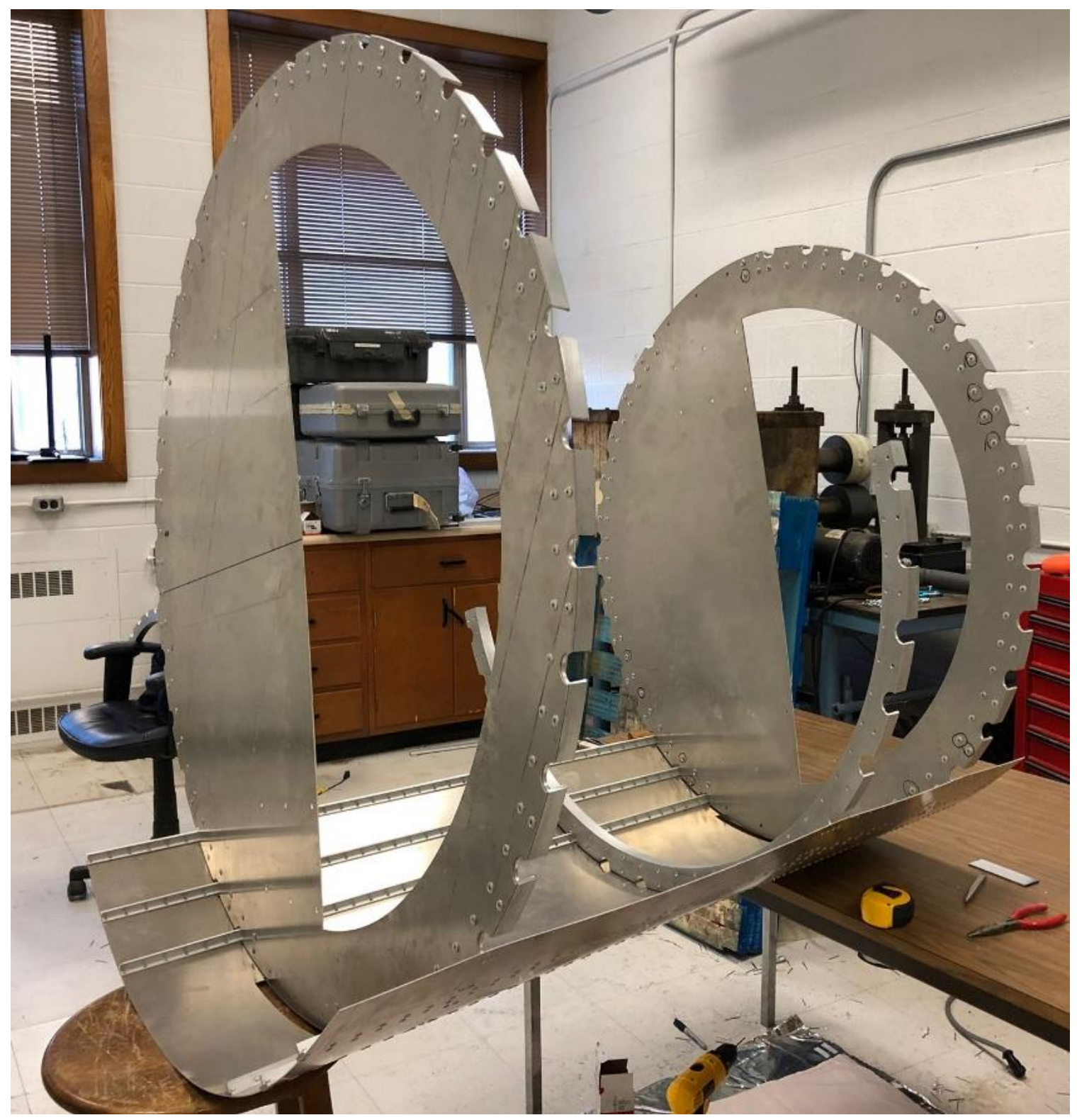

Figure 3.20: Initial Stages of sub-assembly 2 construction. In this image 3 of 4 frame sections including both engine support frames have been attached to one panel of skin. 


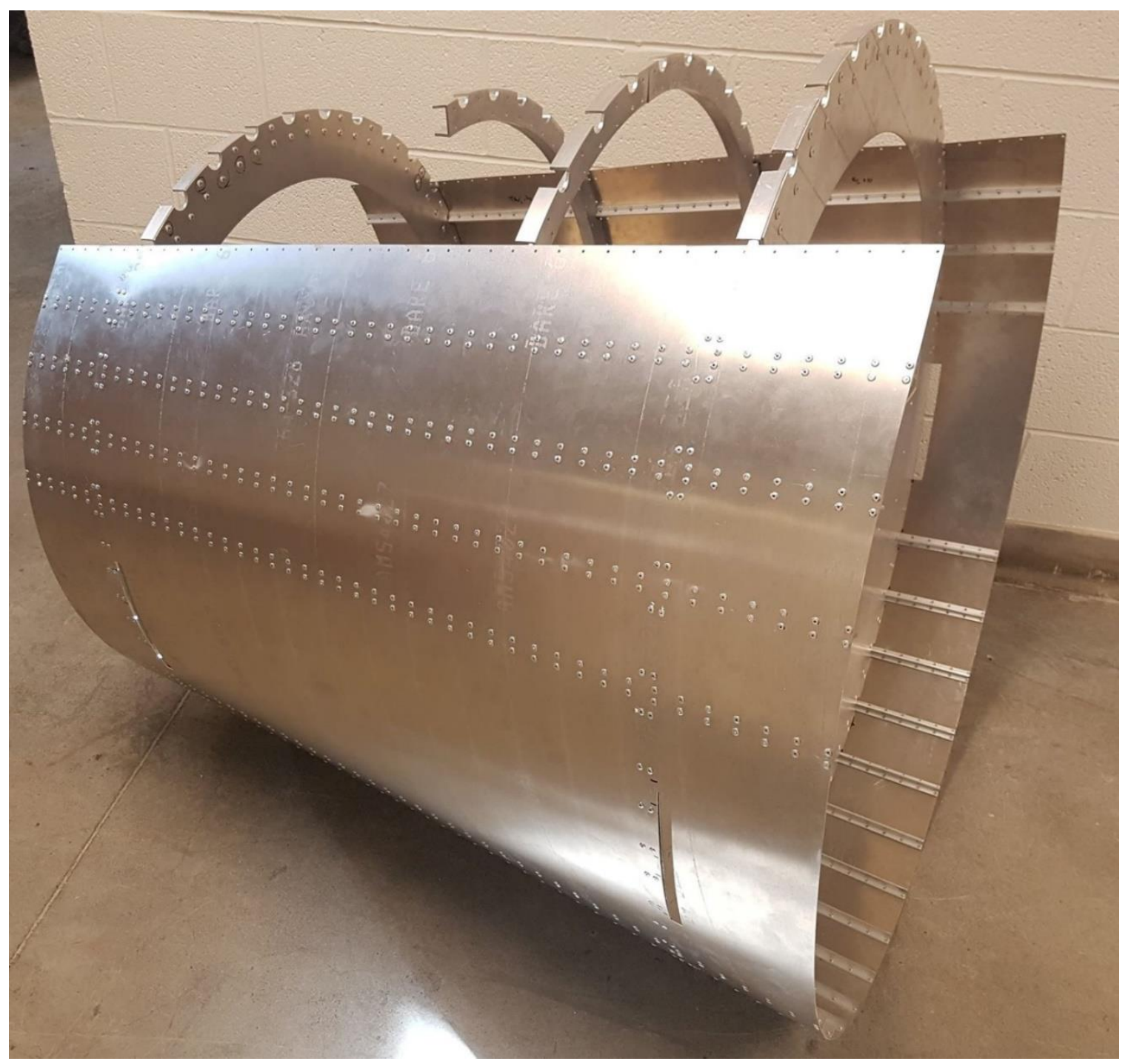

Figure 3.21: The second sub-assembly nearing completion. The slots in the skin form clearance holes for the engine pylons which were added later. 


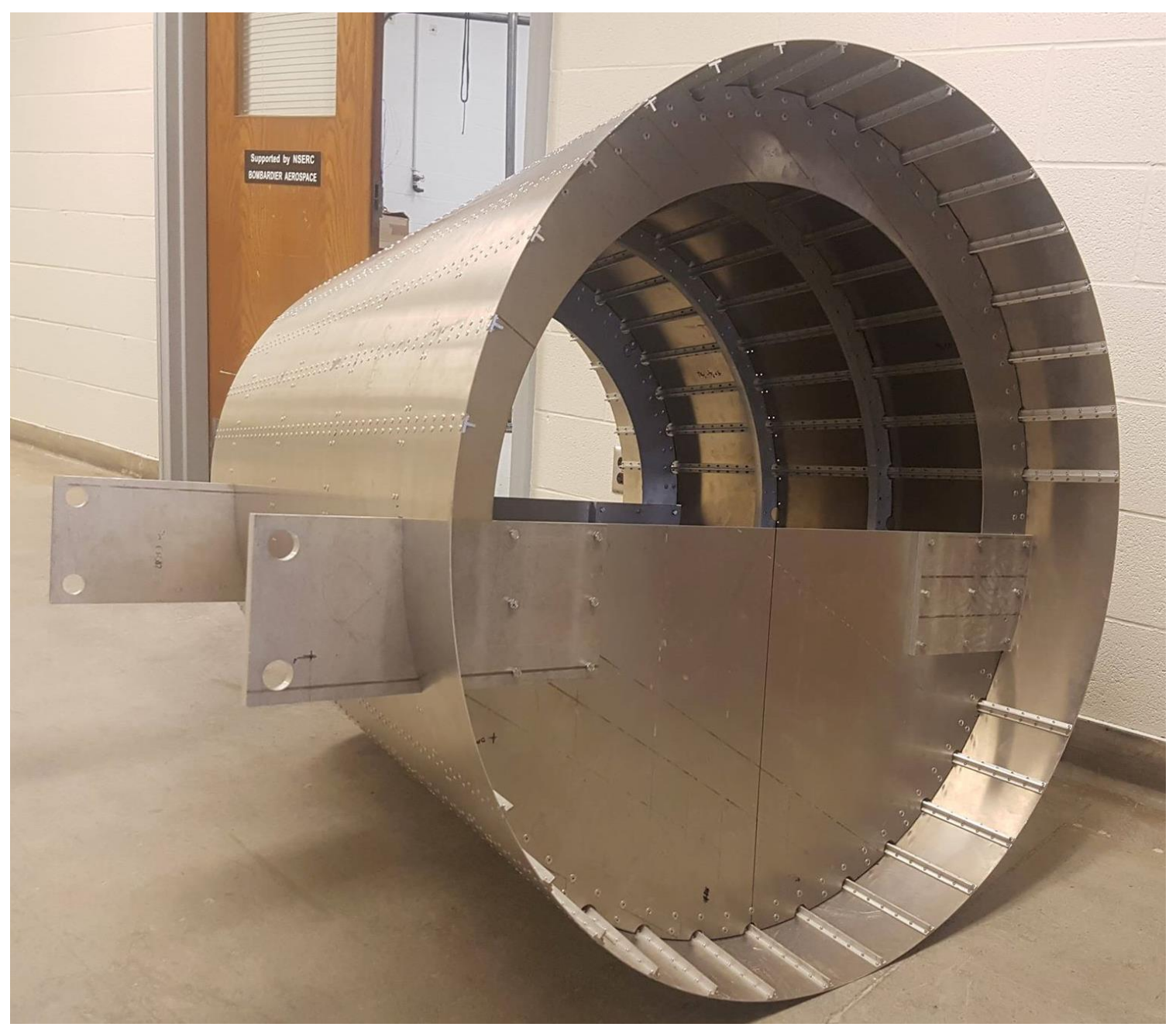

Figure 3.22: The completed second sub-assembly.

\subsection{Full Tail Section Assembly}

The next stage of this project is to evaluate the modal properties of the complete half scale fuselage tail section. To accomplish this, a physical model of the fuselage from the pressurized bulkhead to the aft-most part of the plane must be constructed. This assembly aims to provide a complete set of results with respect to the transmission of sound and vibration from the jet turbines to the passenger cabin through the fuselage structure. These results will help to validate computational models, and these validated models can then be used to guide future structural improvements. This assembly can then provide a platform on which component 
modifications can be evaluated as well as other modifications including the implementation of sound absorptive materials.

The rough design for this assembly was created by Chamberlain and forms a simplified half scale model of a fuselage tail section (Chamberlain, 2018). Some modifications were made to the original design primarily to accommodate manufacturing limitations, and details were added to complete the design for final assembly. The skin was divided into 15 separate panels such that each could be cut using the available waterjet machine. These divisions were chosen such that longitudinal seams lined up with stringers, and seams running around the structure lined up with frames to ensure a strong mechanical connection between each section of skin. To accommodate the transition from a cylindrical to conical shape at the pressurized bulkhead, a frame was added to the front side of the bulkhead. This was done to provide a strong mechanical connection between two independent sets of skin and avoid the complex process of forming the skin over this transition. This modification carries the added benefit of making the front end of the tail section modular, and as a result changing the pressurized bulkhead becomes much simpler. This change is shown in detail in Figure 3.23 through Figure 3.26 which show the final design for the completed assembly. 


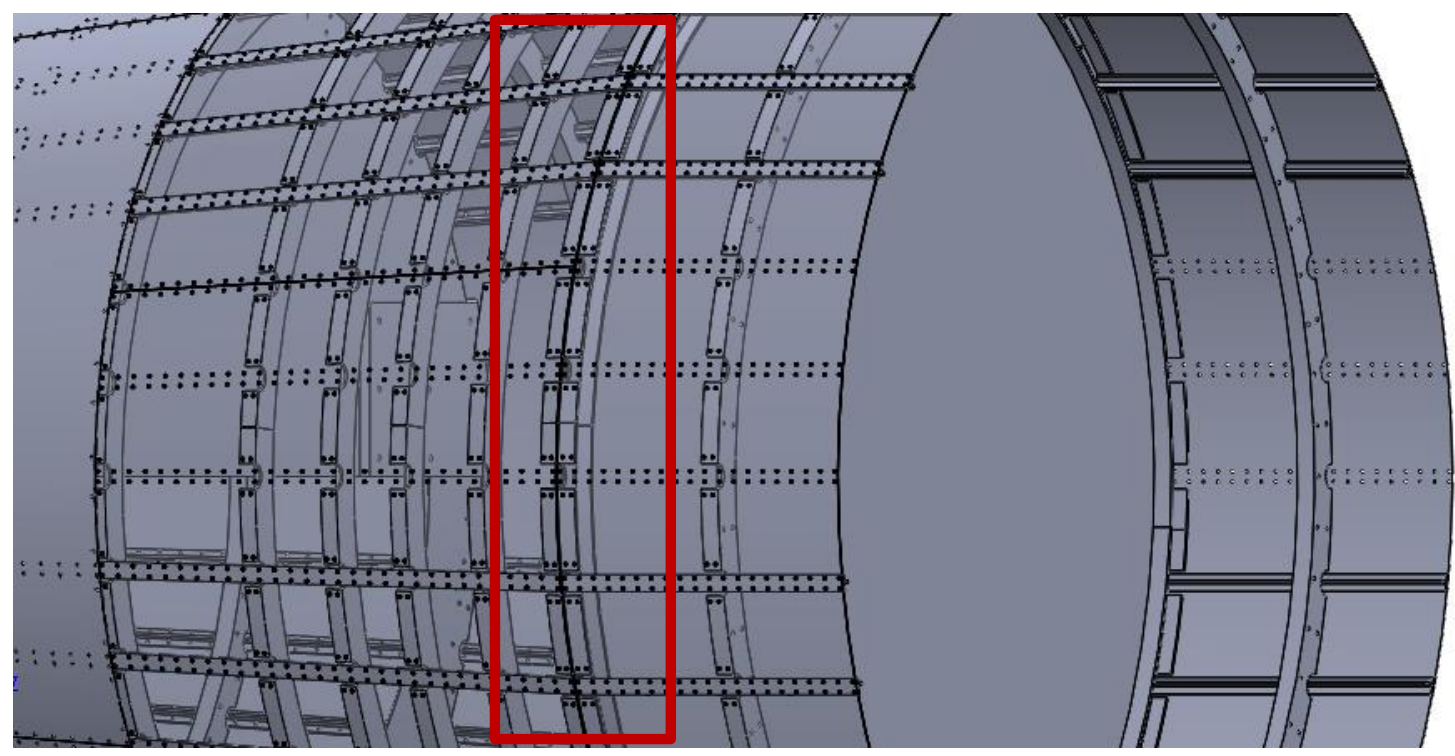

Figure 3.23: The rectangle highlights the frame added to the front of the bulkhead to facilitate the transition between cylindrical and conical skin sections.

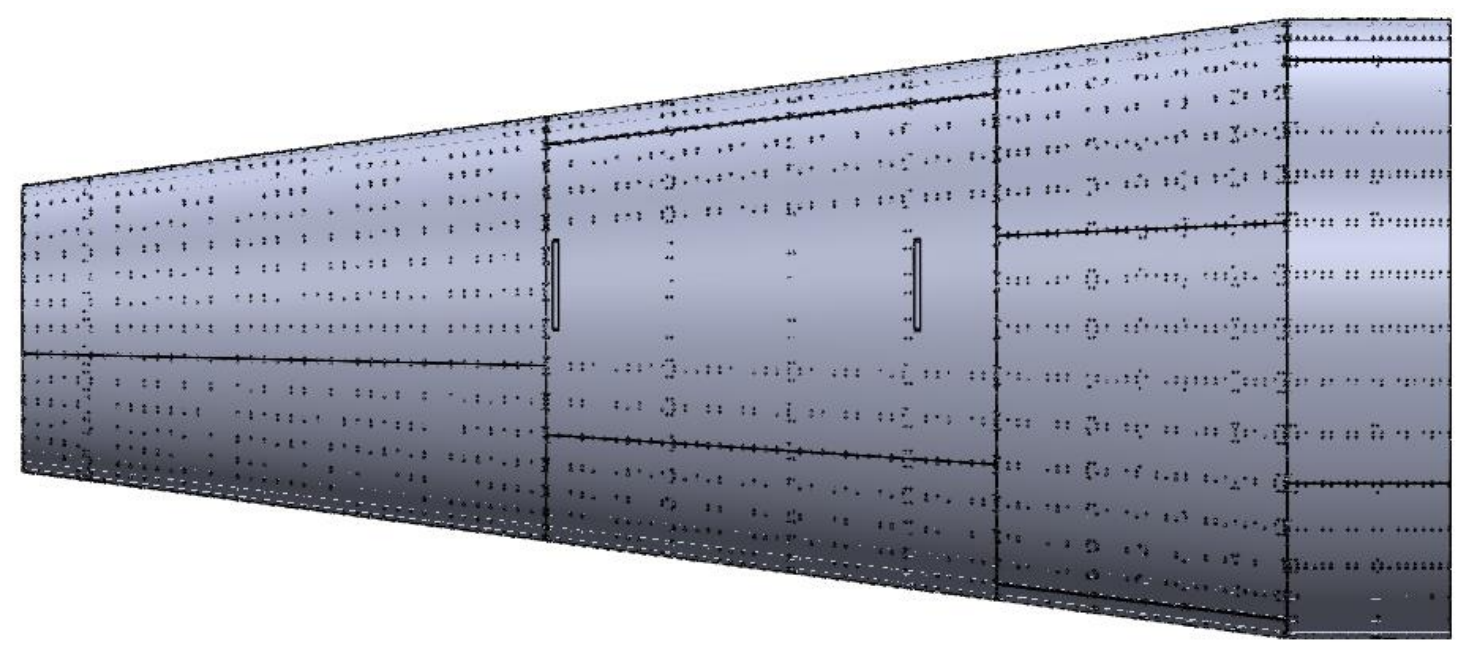

Figure 3.24: Side view of the completed design of the full tail section. 


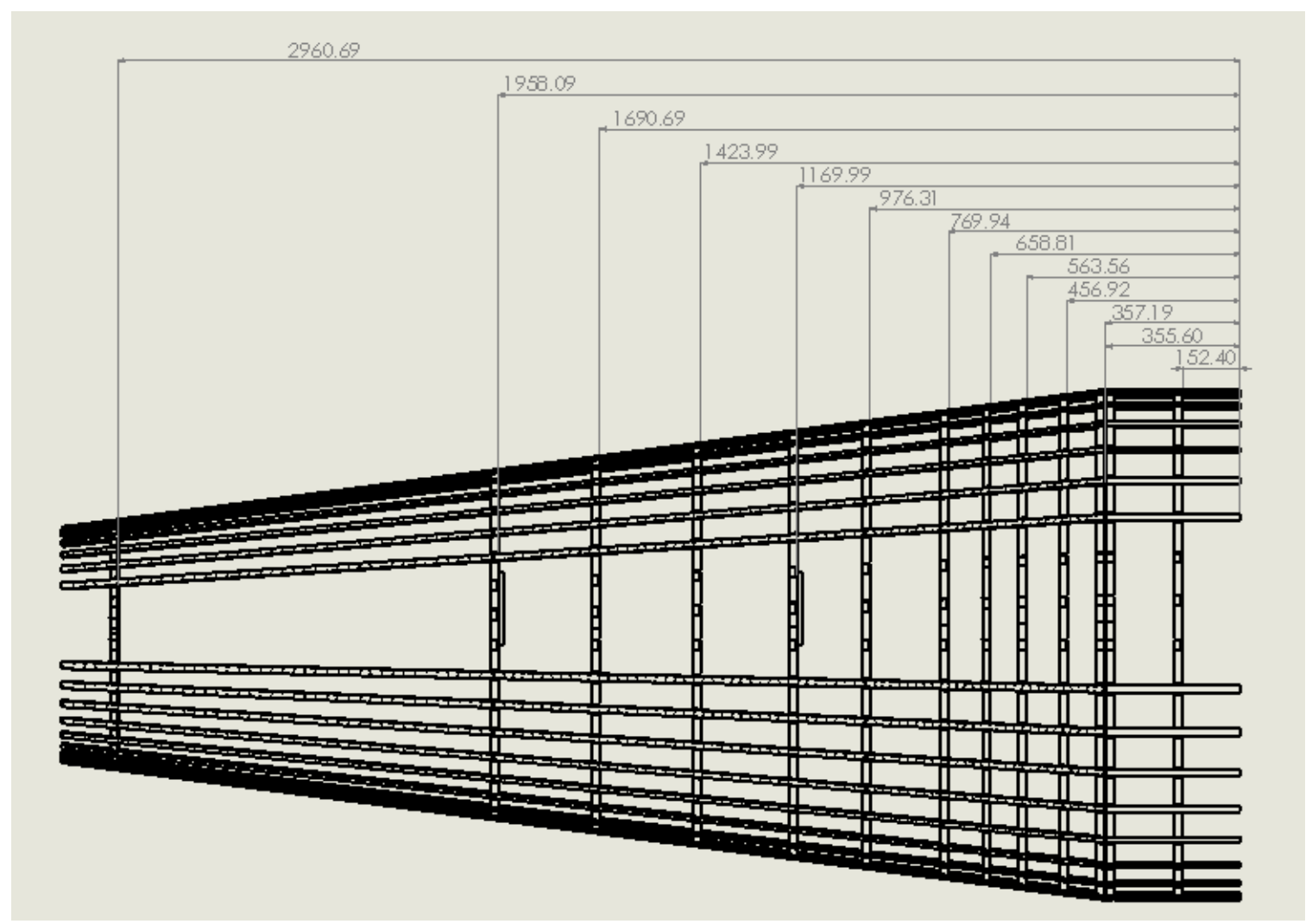

Figure 3.25: Schematic of the internal structure of the full tail section (units in mm).

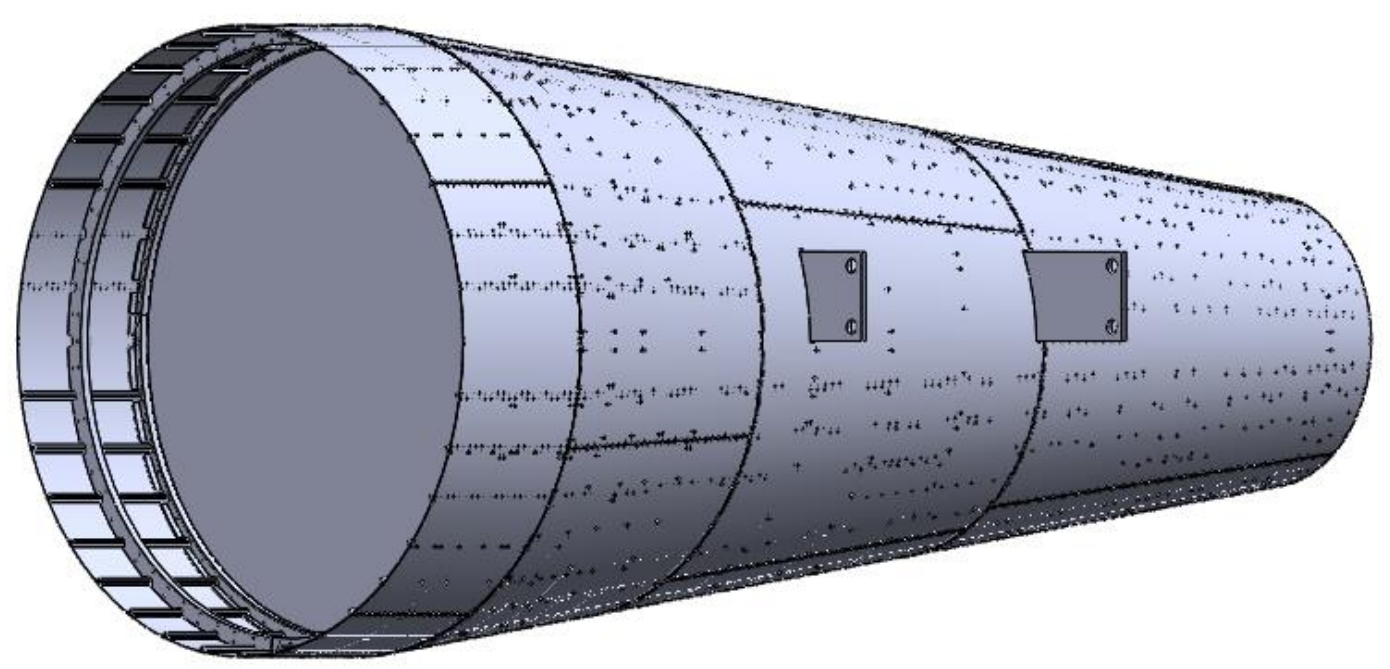

Figure 3.26: Completed design of the full tail section sub-assembly.

Techniques were learned during the manufacturing process of sub-assemblies one and two that will help expedite the construction of the full tail section assembly. On the first version of the first sub-assembly, the holes for the stringers were not cut on the waterjet. This was done to 
reduce cost, as there is a significant financial penalty associated with the time spent using the waterjet machine. This resulted in a significant amount of time drilling these holes into each skin section by hand, and although care was taken, the result could have been more precise. For the subsequent assemblies, the stringer holes were cut on the waterjet, but due to a lack of confidence in the circularity of the frames, the frame holes were omitted until the final assembly of each structure. This became a significant problem during the construction of the second sub-assembly, where it was quite difficult to orient the frames in the correct location. If the holes for the frames were pre-cut in the skin, this procedure would have been easier. It is therefore recommended that all necessary mounting holes for each section of skin are pre-cut, and while this adds cost with regards to time spent on the waterjet, the final result will be easier to assemble and more precise.

\subsection{Chapter Summary}

In this chapter, a detailed description of both sub-assemblies including their design and construction was presented. The design of the individual components was provided, and where necessary, the manufacturing methods used to create them was described. The design revisions implemented on sub-assembly 1 were also presented, and further recommendations for future manufacturing processes were included. Finally, the designs for the full tail section assembly was showcased, including some necessary modifications to facilitate more expedient manufacturing. With an in-depth review of both test articles (sub-assemblies 1 and 2) completed, it is now suitable to discuss the experimental methodology used to test them in Chapter 4. 


\section{Chapter 4}

\section{Experimental Methodology}

\subsection{Overview}

The following chapter describes the experimental setup and methodology used to extract the modal parameters from both sub-assemblies. Additionally, this chapter describes the data processing steps used to convert the raw data output into a form which could be compared with the finite element model (FEM). Multiple sources found in the literature were used to guide the experimental procedure such that it was in accordance with generally accepted best practices. The work by Avitable was used as a guideline of the general practices to follow to complete a successful modal test, and was used during the early phases of test planning (Avitable, 2018). It should be noted that due to differences in test structures and available equipment, no modal testing methodology will apply universally. During the preliminary testing of both subassemblies, a certain level of trial and error was used to determine the optimal testing parameters for the experiments presented. The decisions made during this process were guided in part by random experimentation, but mostly by other sources found in the literature. These references provide solutions more specific to this testing application and will be cited where appropriate in the following sections.

\subsection{Boundary Conditions}

There are two primary methods available when considering the support of a structure during a modal test. The first option is to test the component in a rigid support structure which is often designed to replicate the component's operating condition. This method is also implemented when considering components as part of large structures which cannot be moved, and as such must be tested while fixed in place. Replicating the operational condition of a component is often an arduous task, and for large components such as the fuselage sections tested 
in this thesis, construction of a rigid support would not be feasible as a result of multiple factors including cost and available testing space. It is therefore more common to implement a "freefree" boundary condition for which the primary goal is to decouple the structure from the surrounding environment. It is not possible to support the structure in a truly free-free condition, as its mass mandates the use of some form of support structure. To approximate this condition as closely as possible, soft springs or similar elements are used to form connection between the test article and an external support structure. In doing so the structure is supported in equilibrium on spring elements which allow it to oscillate in space along 6 degrees of freedom. These 3 translational and 3 rotational oscillations form the 6 rigid body modes (RBMs) which according to Avitable should be separated from the low frequency flexural modes by a factor of 10 to reduce dynamic coupling between the RBMs and the flexural modes (Avitable, 2018). To reduce the frequency of the RBMs, the softest possible springs or bungees should be chosen to support the structure. However, a balance must be found between the frequency reduction of the RBMs, and the structural requirements imposed by the mass of the component to be supported. This latter criteria is the first priority, especially when testing heavier objects such as the second subassembly, because a failure of the elastic supports could result not only in severe damage to the structure an experimental equipment, but also pose a safety hazard to those near the structure.

To support the first sub-assembly, two bungee cords were used to connect the fuselage section to a steel structure resting on the ground. These bungee cords were connected to the subassembly through the skin-frame interface via eye bolts. This location was chosen because it provided a structurally sound connection point and held the structure in a suitable orientation to facilitate shaker connection. This location had the added benefit of being modally inactive over the frequency range of interest, which acted to further reduce the dynamic coupling between the RBMs and flexural modes. It was found that adjustability to the height of the structure was required to allow for better alignment with the shaker. To accomplish this, adjustable length ropes 
were created and used to connect the eyebolts and the bungee cords. This allowed for a fine level of adjustment to the height of the structure and thus better alignment with the shaker. Figure 4.1 illustrates the boundary condition setup and support structure used during experimental testing of sub-assembly 1.

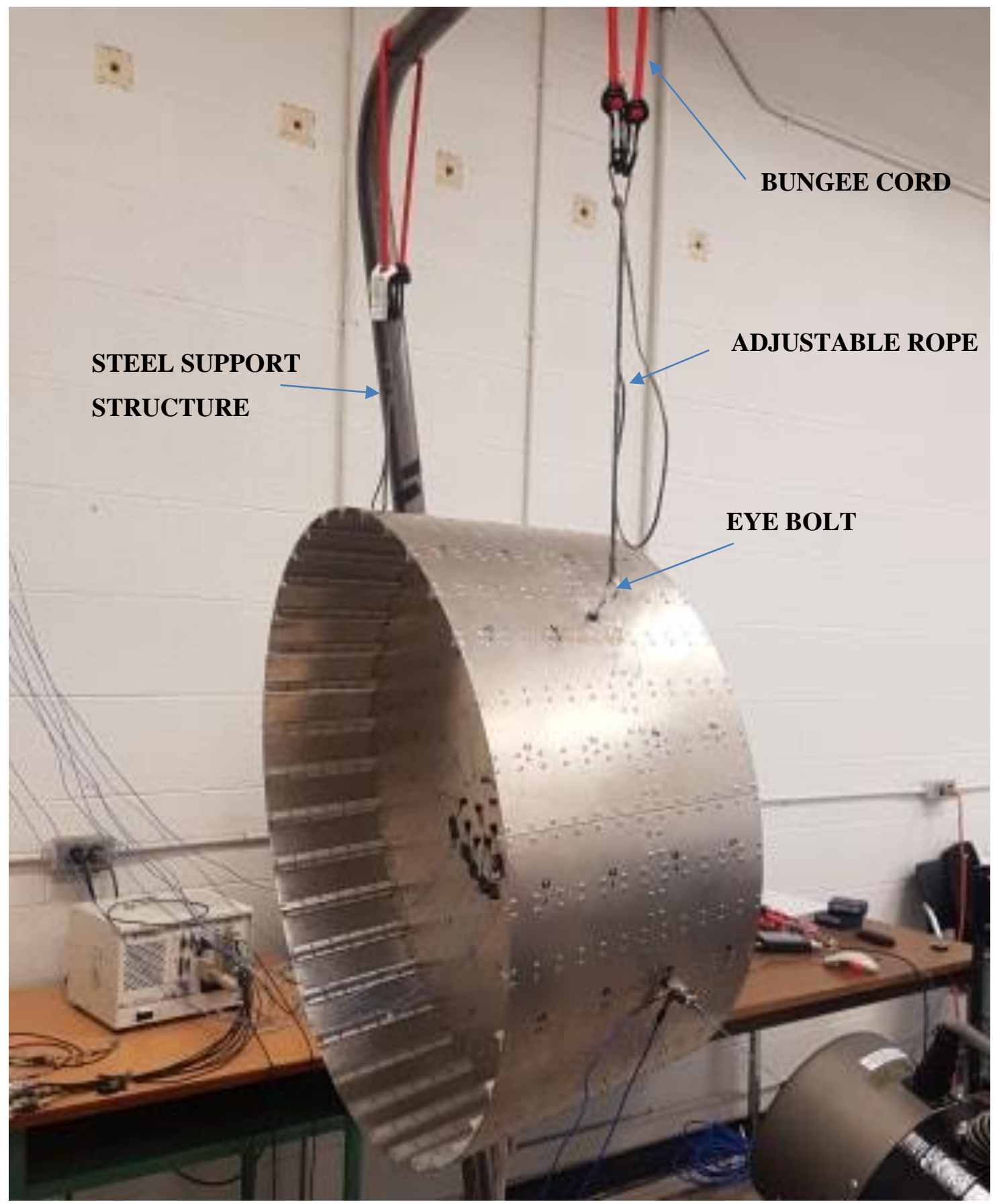

Figure 4.1: The boundary condition setup used for sub-assembly 1 
The second sub-assembly was approximately 4 times the size and weight of the first and required stronger bungee cords attached at four locations around the structure. Due to the larger size of this structure two steel support frames were used to provide a more stable test setup which to some extent aided with repeatability, but above anything else provided a safe working environment. Once again adjustable length ropes were used to connect the structure with the bungee cords, however instead of using eye bolts, carabiners were fastened to existing holes in both engine support frames. This was done partly for convenience, but also to minimize any unnecessary modifications to the structure. As a result of the stiffer bungee cords, and their attachment to modally active sections of the structure, there was a higher risk of the flexural modes being affected by the boundary condition. These effects will be discussed in more detail in chapter 5, where future improvements to this experimental setup are also proposed. Figure 4.2 and Figure 4.3 illustrate the support structure and boundary conditions used to test sub-assembly 2. 


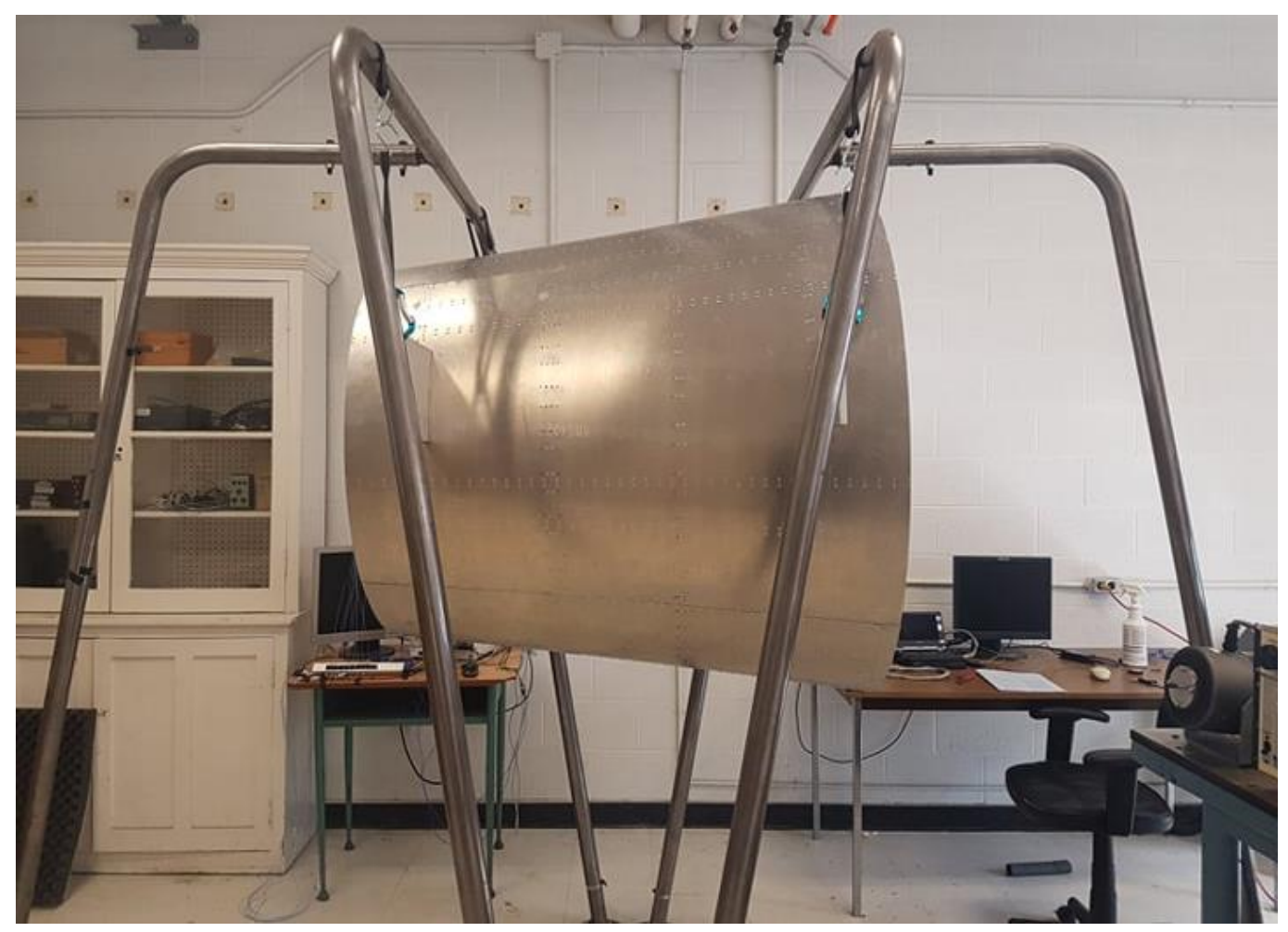

Figure 4.2: The support structure used for sub-assembly 2.

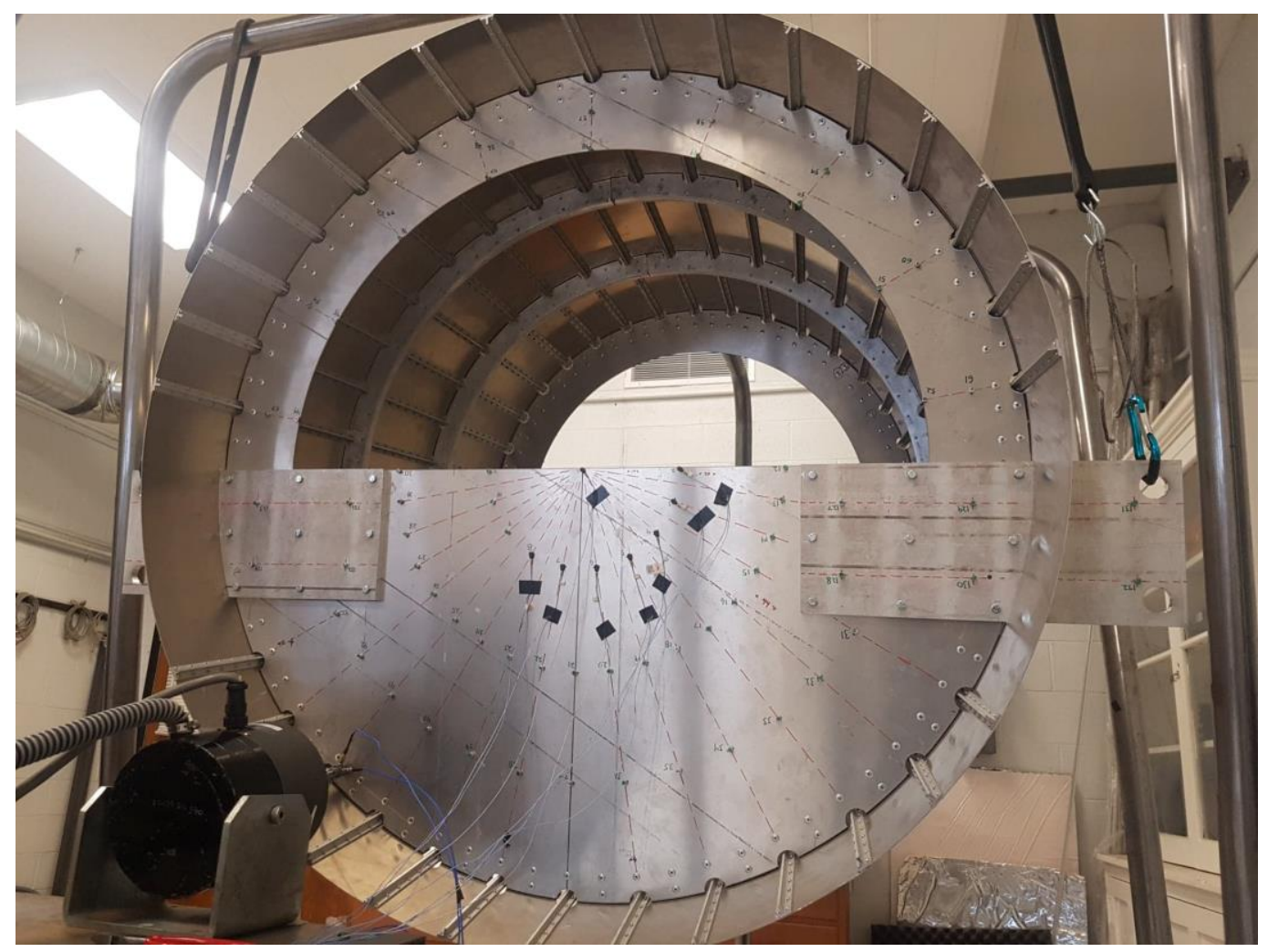

Figure 4.3: Boundary condition setup for sub-assembly 2. 


\subsection{Excitation Methodology}

When considering the excitation of a structure during a modal test, there are multiple methods available to choose from. In some cases, the operating conditions of a structure are used as a form of natural excitation, which yields some information regarding the dynamic response of the structure. However, because the force input is not measured, there is no way to ensure excitation of all resonant frequencies across the bandwidth of interest. Additionally, with no force input measurement, a calibrated FRF cannot be obtained, and the mode shapes cannot be scaled (Avitable, 2018). As such, for structures being tested in isolation, excitation methods for which the input force can be measured are preferred. Of these there are two techniques available, the modal impact hammer and a modal shaker. There are many benefits and drawbacks to both methods, but for the case of testing large structures in a stationary lab setting, the shaker table was chosen. According to (Avitable, 2018), "Shaker testing allows for a much more uniform excitation of the structure, which is very important when testing larger structures." The experimental setup of the modal shaker will be focused on in this section, however for more information on the experimental setup with regards to an impact hammer, previous work on experimental modal analysis of individual aircraft components as documented by Chamberlain should be referenced (Chamberlain, 2018).

The shaker used was a Labworks ET-139 modal shaker. This device includes a through hole armature as well as a trunnion base, both of which provide adjustability to make alignment easier during setup. This shaker could provide more than enough excitation force, with a peak sine force of $75 \mathrm{lbs}$ and a peak random force of 50lbs. A smaller unit would have been equally suitable, however due to its availability in the lab, this shaker was chosen. The shakers can either be clamped to a rigid structure or suspended near the test structure on a stand. Both methods are capable of delivering excitation to the test article, however clamping the shaker down to a large relatively immobile structure was found to provide a more consistent test setup. An image of the 
shaker arrangement used during the modal parameter extraction of sub-assembly 2 is shown in Figure 4.4 below.

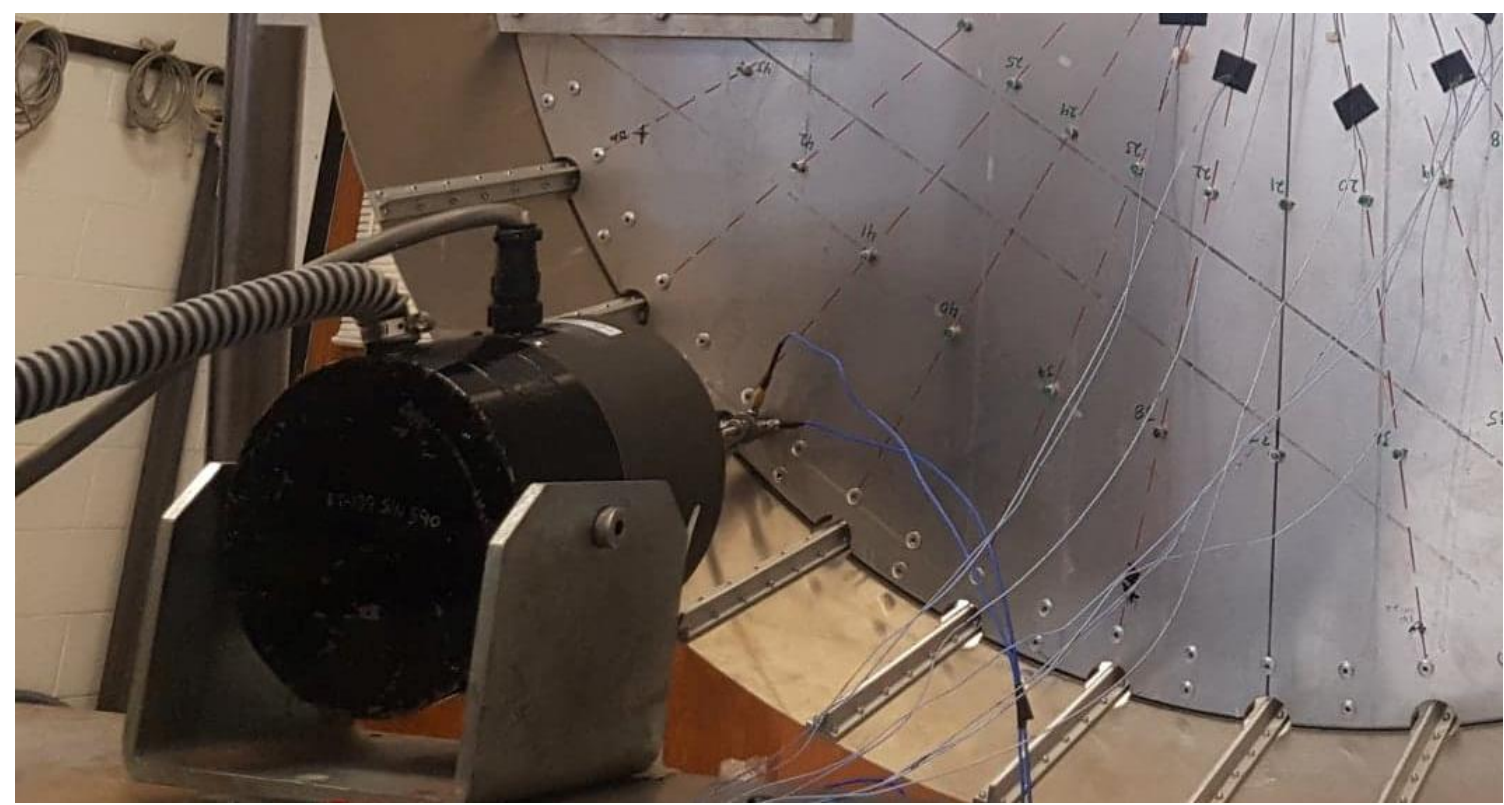

Figure 4.4: Shaker setup during sub-assembly 2 modal testing

There is an array of different excitation signals which can be used in conjunction with a modal shaker to excite the structure. Each have their benefits and limitations and can be divided into two general categories: deterministic and non-deterministic signals. Deterministic signals such as sine sweep and sine chirp increment through the frequency range of interest from user defined lower and upper bounds. They are defined by a mathematical function, and as such will provide the same excitation throughout the averaging process. As a result, they are ideal for determining the response linearity of a structure (Avitable, 2018). The main advantage to sine chirp is that a complete sweep of the frequency range is completed within one sample interval. This allows for the response to decay, and in conjunction with a pre-trigger delay, the periodicity requirement of the FFT can be satisfied without the use of windows. For these reasons, a sine chirp signal was selected to evaluate the linearity of both structures during the linearity validity checks. 
Non-deterministic signals including pure random and burst random do not conform to any mathematical function. They therefore provide different excitation throughout the averaging process and will average out noise and slight non-linearities from the results. However, it is important to determine the overall response linearity using a deterministic signal, before proceeding with the assumptions made for linear modal analysis. Both structures were found to behave in a linear manner, however, slight noise and non-linearities were found as seen in Figures 5.2, 5.3 and 5.4 so a burst random signal was chosen for modal parameter extraction. This signal has the same benefits as sine chirp, in that the signal is contained within one sample interval so in most cases no windowing is required, which is why it was chosen over other non-deterministic signals. To generate the excitation signals for each test, an LDS Dactron Photon 2.0 was used in conjunction with RT Pro software (LDS-Dactron, 2004) (Bruel \& Kajer, 2008).

The shaker was powered by a Labworks PA-141 Linear Power Amplifier. This device could be used as a highly damped voltage source or as a high impedance current source. There are benefits to both operational modes, but when using waveforms which are meant to decay in the sample interval, the voltage source mode is preferred. Using this mode provides resistance to the armature in the shaker after excitation which helps the input signal decay to zero more quickly. This resistance gets recorded by the impedance head transducer on the structure, so the results are not contaminated, and the increased decay rate helps to eliminate the need for windows (Peres, Bono, \& Brown, 2010). This meant that any signal distortion that might come as a result of windowing was not present in the results produced by this experiment.

Another factor to consider when using an impact shaker is the method of coupling it to the structure. It is of course essential to measure the input force into the structure to collect enough information to provide calibrated FRF plots. There is however a complication associated with this measurement, as typical force transducers or impedance heads used in experimental modal analysis (EMA) only measure force in one axis. It is therefore essential to excite the 
structure along this axis, while minimizing all other inputs to the structure which would contaminate the results. This is done by using a connecting rod or wire known as a stinger, which directs force along one axis, while being flexible in all other directions as to not impose stiffness effects on the structure.

There are multiple types of stingers which effectively reduce the out of axis forces applied to the structure. A piano wire stinger accomplishes this most effectively, as the lateral stiffness of the wire is minimal, however the wire must be pre-loaded with a force greater than the applied force to the structure to avoid buckling during testing. This adds more complication to the test setup, and for structures suspended using a free-free boundary condition a piano wire is often not feasible. The most commonly used stinger is a thin "drill rod" which negates the need for applied pre-load and is viewed as an acceptable compromise between performance and usability (Peres, Bono, \& Brown, 2010). As such a drill rod stinger was selected for use during the experiments presented, however the added lateral stiffness has the potential to reduce the quality of the results. The effect of the stinger's lateral stiffness on the results is largely dependent on the stiffness of the structure as well as the frequency range being tested. For relatively stiff structures being tested at low frequency ranges $(<1000 \mathrm{~Hz})$ the lateral stiffness of the stinger is not as critical, however this needs to be validated on a case by case basis. According to Peres et al. an effective way to evaluate the stingers lateral effects on the structure is to vary the length of the stinger by $+/-10 \%$ and observe any changes in the measured frequency response functions (Peres, Bono, \& Brown, 2010). This procedure was included as part of the pre-test validity checks and the results are presented in Chapter 5. Figure 4.5 illustrates a typical drill rod stinger forming a mechanical connection between a modal shaker and test structure. 


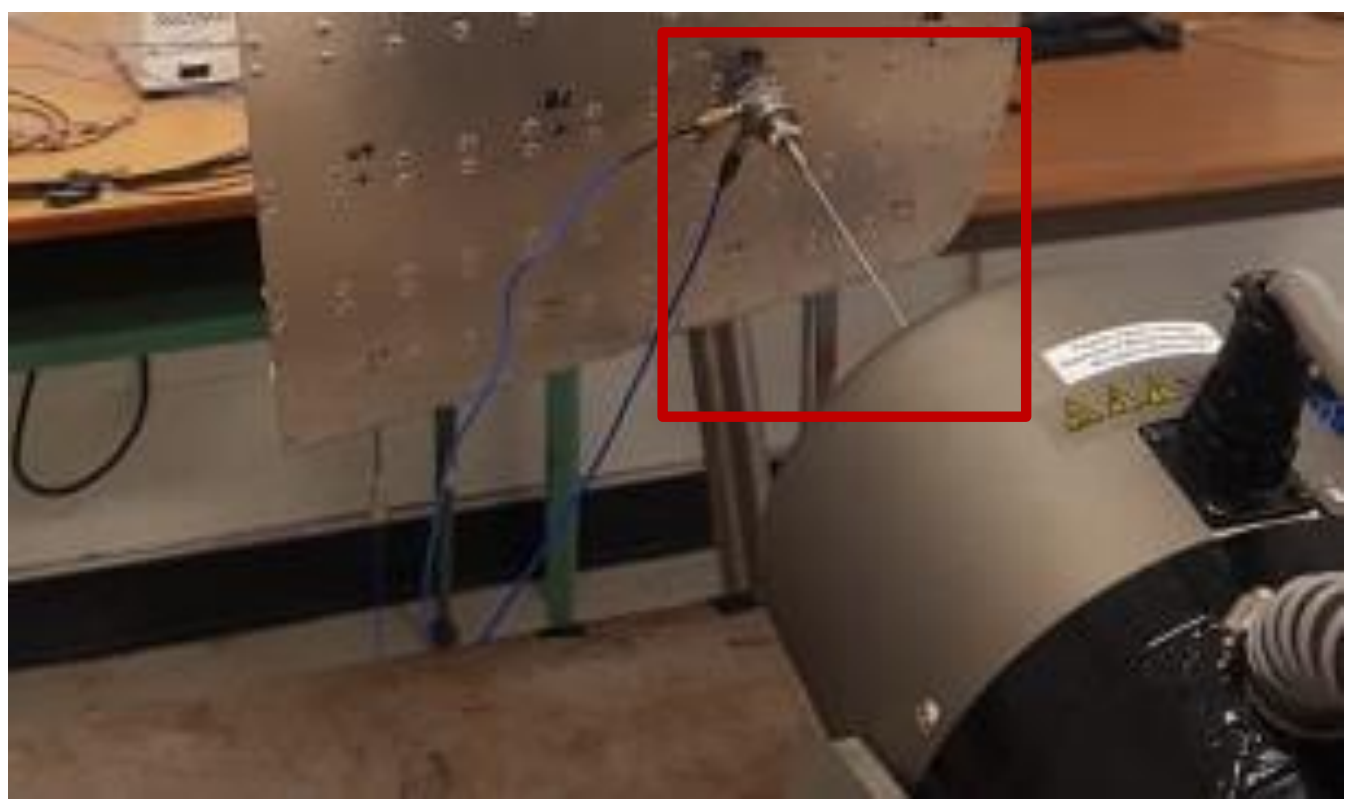

Figure 4.5: Typical drill rod stinger setup implemented during preliminary testing of subassembly 1 .

\subsection{Input and Output Measurements}

To collect FRF curves during a modal test, the input force must be measured. Previously this was accomplished using a force transducer, however this creates an issue regarding the driving point measurement (Peres, Bono, \& Brown, 2010). Significant error in this measurement is associated with misalignment of the force transducer and accelerometer. An impedance head integrates these sensors into one unit thus ensuring perfect alignment and has the added benefit of providing a driving point measurement for each set of measurements performed throughout the experiment. To collect the force input and driving point measurements, a PCB Piezotronics 288D01 impedance head was used (PCB Piezotronics, 2020). The impedance head was attached to the structure by first adhering the supplied adhesive mounting base to the structure, then attaching the sensor via an intermediate stud. For permanent installations it is also possible to attach the sensor directly to the structure assuming a hole for the stud can be drilled and tapped in the appropriate location, however for the experiments performed the adhesive base was sufficient. A schematic of the impedance head and adhesive base is shown in Figure 4.6. For a more detailed 
drawing as well as product specifications reference the PCB Piezotronics website (PCB

Piezotronics, 2020).

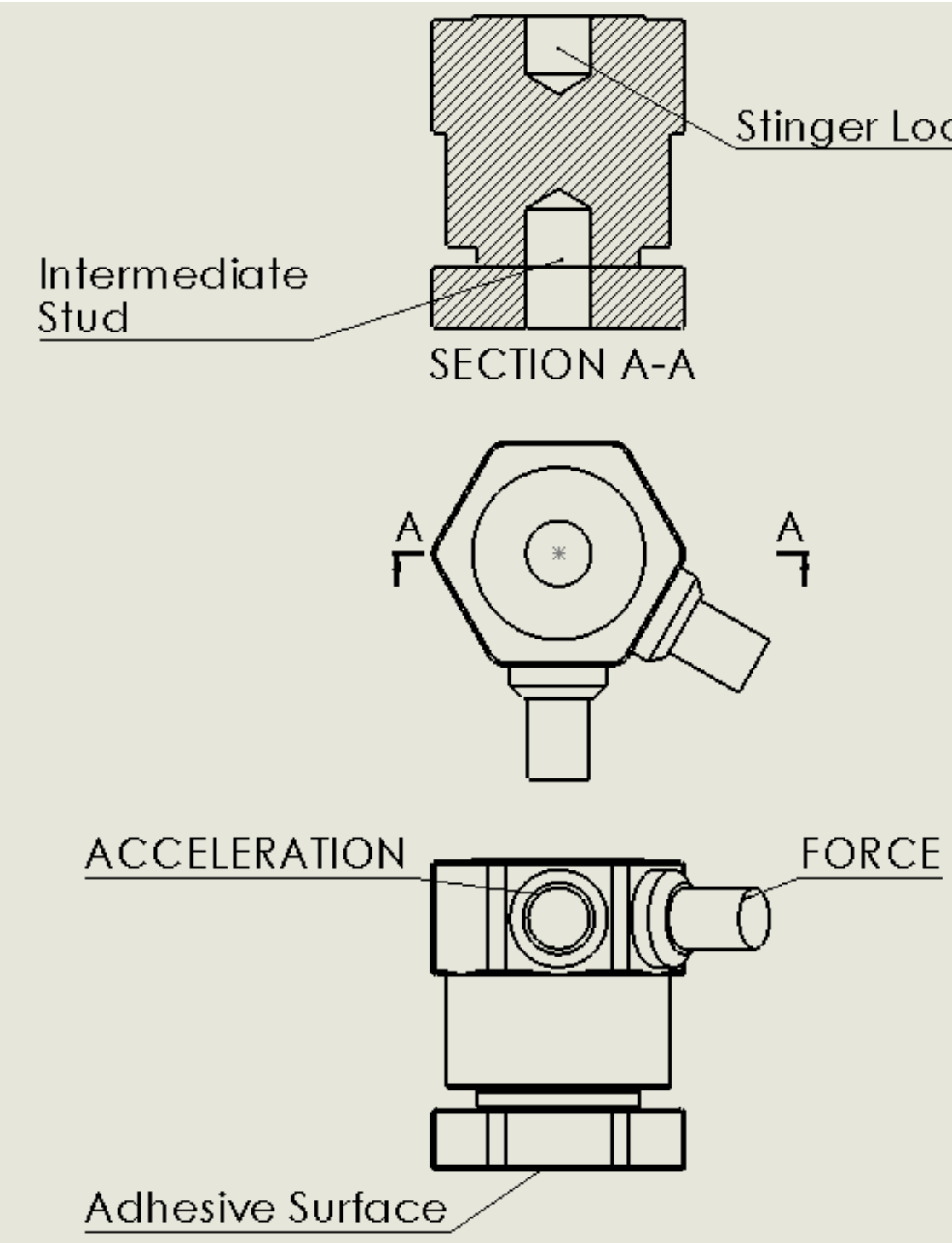

Figure 4.6: PCB Piezotronics impedance head 288D01.

The selection of a suitable excitation location is essential to the success of a modal test.

Factors to consider when selecting a driving point include accessibility, the ability to excite all modes in the bandwidth of interest, and the ability to excite the entire structure. The first consideration is trivial, as the point chosen must be accessible for attachment with the shaker stinger apparatus, which in this case limits the available options to exterior surfaces of both 
fuselage sub-assemblies. To excite all modes in the bandwidth, first the location of modal nodes must be considered. As stated in Chapter 2, excitation at the node of a certain mode $\mathrm{N}$ will result in little to no response, greatly reducing or eliminating the presence of a resonant peak in the FRF data. The most common way to avoid a node is to excite the structure in a modally active region, however this can create other issues when considering flexible structures. If the shaker is attached to the structure where the response displacement is high, it will not be able to force the structure over its entire range of motion. This will cause a drop in the force spectrum at one or more resonances, which will result in poor frequency response measurements (Avitable, 2018). In this situation exciting the structure in a stiffer location will provide more even excitation across the frequency range and can also assist in exciting the entire structure more evenly. To find a suitable driving point location, multiple tests were performed on sub-assembly 1. Initially the structure was excited through DOF 20 of the aft bulkhead, however this location was not stiff enough to excite the surrounding skin structure. Multiple locations on the skin were also evaluated, however many of the same problems were encountered. Ultimately, it was determined that exciting the structure where the bulkhead connected to the skin as shown in Figure 4.7 provided the best results. The results from this test were considered when selecting the driving point for subassembly 2, which again was located near the connection between the skin and FESF panel as shown in Figure 4.8.

Accelerometers are most often used to measure the dynamic response of a structure during EMA. There is one major factor which much be considered when selecting and using accelerometers, and that is the effect of the accelerometer's mass on the dynamic response of the system. There are a couple of ways to overcome this issue the first and simplest of which is to select lightweight accelerometers. The accelerometers chosen were PCB Piezotronics 352C22, which had a mass of just 0.5g (PCB Piezotronics, 2020). According to Avitable however, "while the accelerometer weight may be very small in relation to the wight of the structure, the weight 
must be considered relative to the effective weight of the structure at the attachment point." (Avitable, 2018). To evaluate the effects of the accelerometer mass on the structure, dummy masses were machined out of aluminum to simulate the weight of the accelerometer on the structure in locations where accelerometers were not present. The structure was tested with and without the dummy masses in place, and it was found that the mass of the accelerometers had a significant effect on the alignment of resonant frequencies as shown in Figures 5.11 and 5.12. To successfully correlate the FEM results with the experimental results, the accelerometer mass must be included in the computational model, which is accomplished by adding a lumped mass to the nodal location of the accelerometer. This simple modification is made complicated when considering a roving response measurement, during which the accelerometers (and their associated mass) move around the structure, resulting in a non-constant mass distribution. The simplest way to mitigate this is to add dummy masses to the DOFs not being measured, such that the distribution of mass is kept constant regardless of accelerometer placement. As such, dummy masses were implemented during testing of both sub-assemblies to eliminate the roving mass effect, which improved the alignment of the resonant peaks due to the global parameters of the system remaining constant.

Another critical consideration during the setup phase is the selection of response measurement locations. The objective of this placement is to provide both complete visual and mathematical representations of each mode shape in the bandwidth of interest. It is also important to consider which DOFs will be required to form a set of mathematically correlated mode pairs between the experimental results and computational model. The expected mode shapes of the structure, and their complexity will for the most part define the number of degrees of freedom required. The computational models developed by (Lam \& Mechefske, 2020). provided an indication of the mode shapes for both sub-assemblies, which in turn guided the placement of measurement locations for preliminary tests. The placement of DOFs for the bulkhead of sub- 
assembly 1 is shown in Figure 4.7 below. Points 1 through 55 were used during preliminary testing, at which point an AutoMAC (as described in Chapter 2) was used to determine if the measurement was sufficient. It was found that more points were required and as such points 129 through 146 were added and the measurement process was repeated. The AutoMAC matrices produced by both tests are presented in Chapter 5 to illustrate the improvement made.

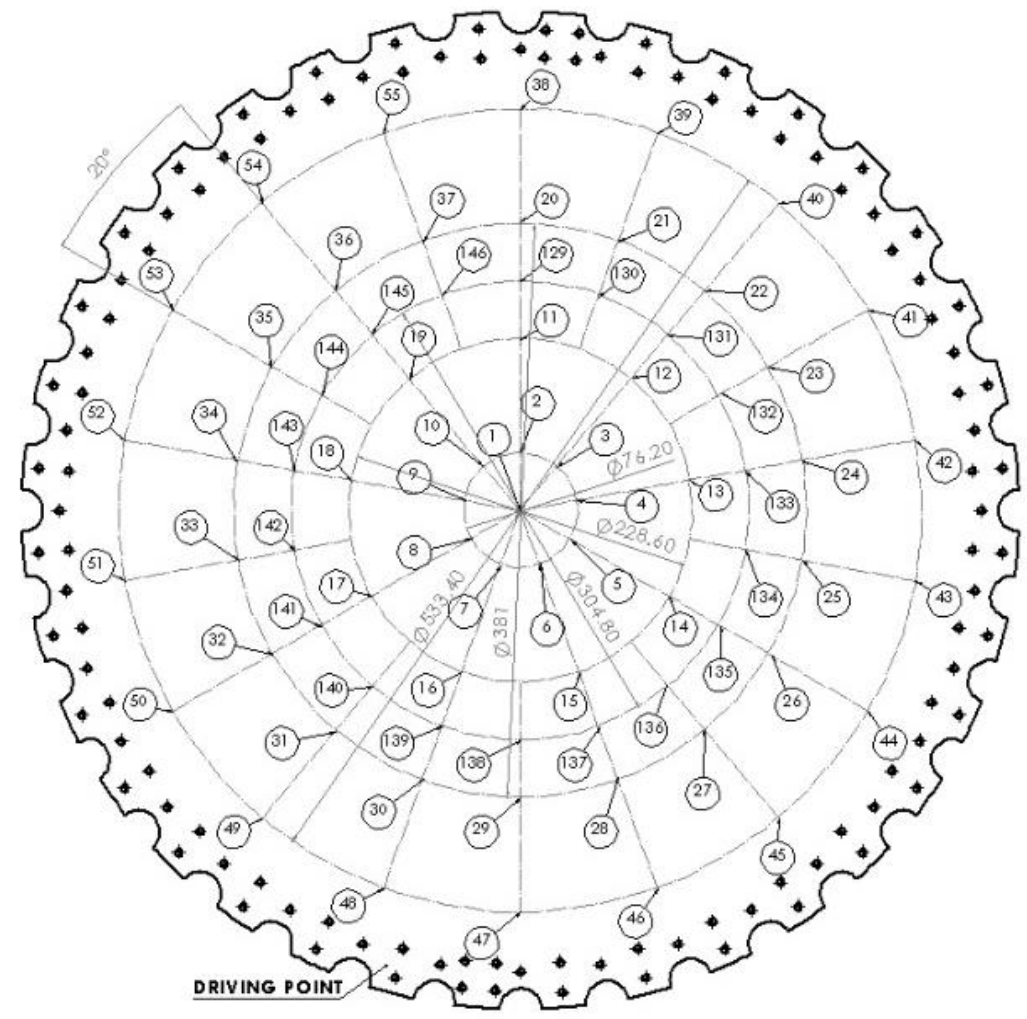

Figure 4.7: Sub-assembly 1 bulkhead DOF placement.

The response measurement locations for sub-assembly 2 were selected using the same method used for sub-assembly 1 . The FEM produced by (Lam \& Mechefske, 2020) was used to guide the placement for preliminary tests, then an AutoMAC was used to provide an indication of the confidence with which each mode shape could be mathematically differentiated. When evaluating the AutoMAC created using the preliminary data, it was found that the initial placement for both the FESF and RESF of the second sub-assembly as shown in Figure 4.8 and Figure 4.9 was sufficient. 


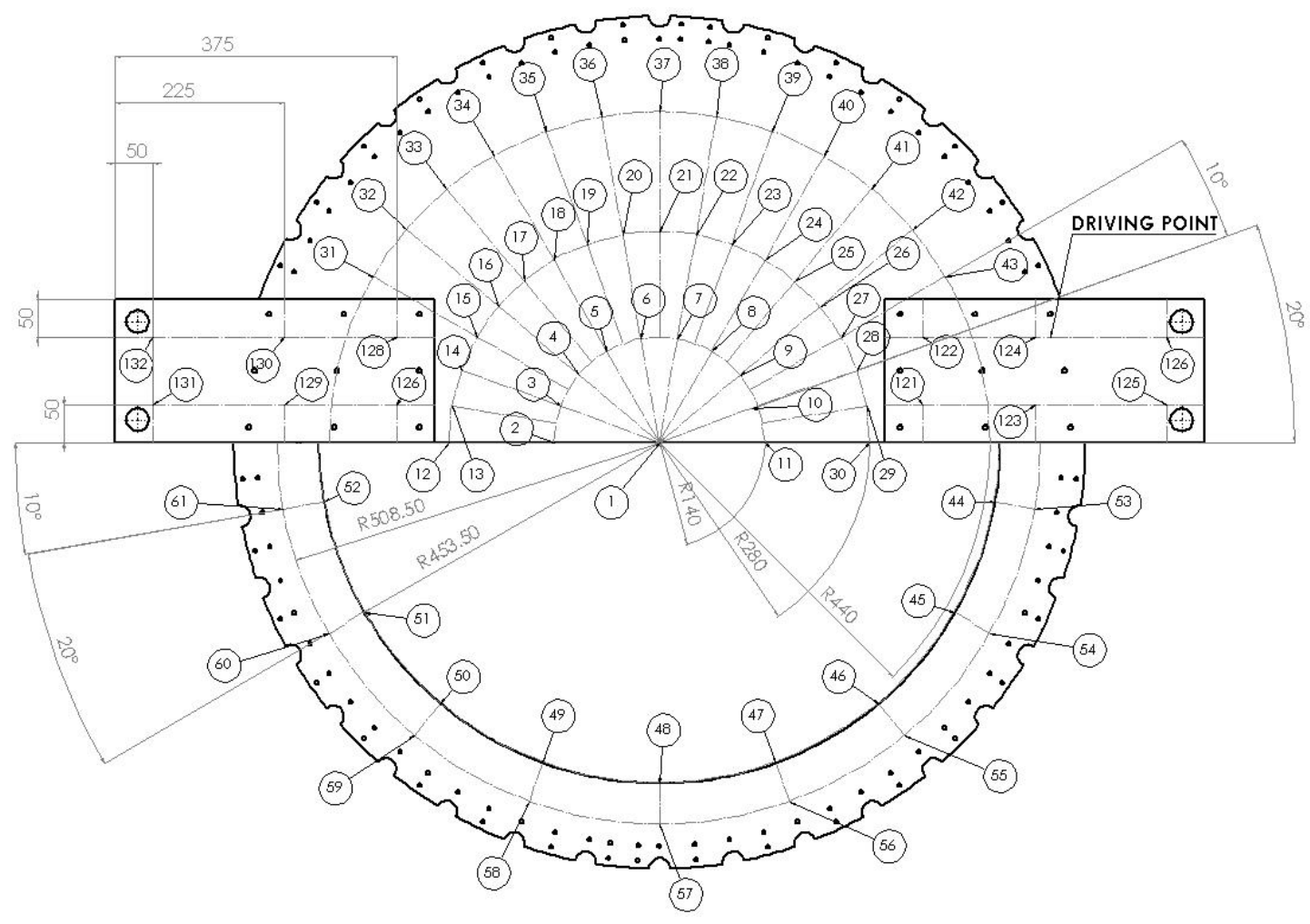

Figure 4.8: DOF placement for the FESF of sub-assembly 2.

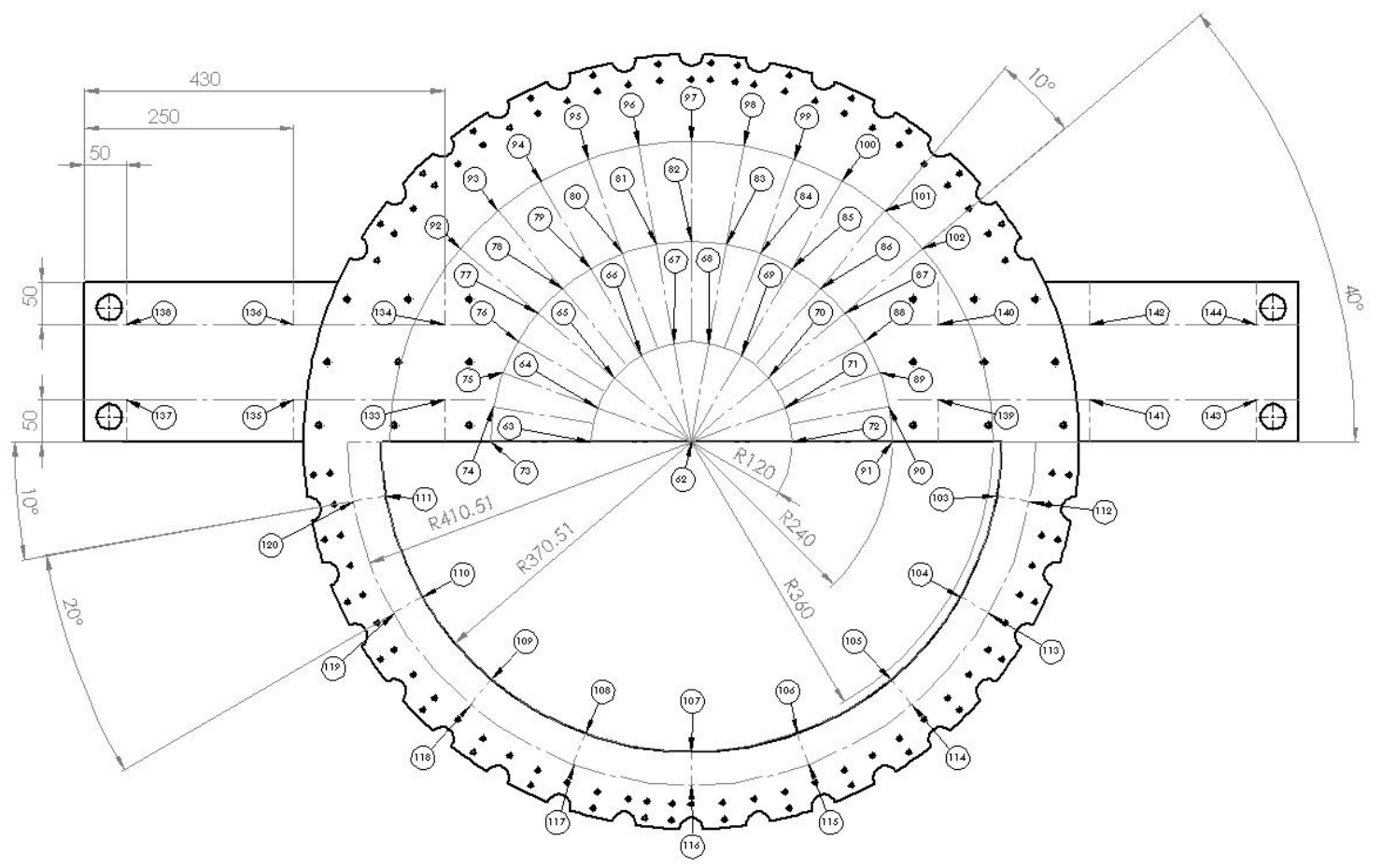

Figure 4.9: DOF placement for the RESF of sub-assembly 2. 


\subsection{Data Acquisition}

The data from the validity checks and full modal experiments was collected using a National Instruments NI PXI-1031 (National Intruments, 2019). This system can collect data from 16 channels, however for the experiments performed, only 10 were used. Two channels were used to collect force and acceleration data from the impedance head, while eight were used to collect response data from the roving accelerometers. Using this number of accelerometers provided a compromise between expedient testing, and the ability to monitor individual accelerometer measurements during the averaging process. Using more accelerometers would have reduced testing time but would have also increased the probability of sensor error going unnoticed during testing.

For the first sub-assembly, a sampling rate of $1000 \mathrm{~Hz}$ was used to collect data over a 2.5 second sample interval. It was found that 2.5 s provided enough time for the structure to be excited fully by the shaker, then have its response decay back to zero in accordance with the periodicity requirement associated with FFTs. This yielded a frequency resolution of $0.4 \mathrm{~Hz}$ for the frequency domain plots, and a maximum bandwidth of $500 \mathrm{~Hz}$. This higher bandwidth was chosen as it was not initially clear what frequency range would be required to confirm correlation between the experimental results and computational model. Additionally, there were not any limiting factors regarding the data storage capabilities, so longer sample intervals could be used to increase the frequency resolution of frequency domain plots. The second sub-assembly used the same sampling rate, but the sample interval was increased from $2.5 \mathrm{~s}$ to $5 \mathrm{~s}$. This was done to give the structure more time to reach steady response and decay, which was required due to its increased mass and response levels at certain DOFs. It also had the added benefit of increasing the frequency resolution to $0.2 \mathrm{~Hz}$ for frequency domain plots. Fifteen averages were taken during modal parameter extraction of both sub-assemblies, which increased the statistical 
reliability of the data, and helped to reduce the effects of noise and slight non-linearities due to the burst random excitation signal (Agilent Technologies, 1997).

Another important factor to consider when configuring the sampling parameters is the pre-trigger delay. This parameter allows for data before the start of each excitation to be stored, thus capturing a near zero response of the structure as well as the complete excitation which otherwise might be missed. Any excitation information that is not collected would likely result in distortion of the FRF data (Avitable, 2018). A pre-trigger delay is especially important when performing impact measurements, and shaker measurements using non-deterministic signals, as it allows for the periodicity requirement to be met for these instances of random input without the use of windows. For the experiments presented, the sample was triggered by the force transducer in the impedance head, and 200 pre-trigger samples were collected.

\subsection{Signal Processing}

The raw analog input and output signals must be processed into useful FRFs which can later be used for modal parameter extraction. This process begins with the application of antialiasing filters, which in this case are low pass filters to prevent high frequency content from contaminating the results in the bandwidth of interest. For most data acquisition systems, the filter parameters are set based on the sampling frequency chosen. A sampling frequency of $1000 \mathrm{~Hz}$ was used during testing resulting in a frequency bandwidth of $500 \mathrm{~Hz}$ in accordance with the Nyquist requirement. The input and output signals are then digitized using a built-in analog to digital converter at which point windows could be applied if necessary. For the experiments presented, no windows were required. The digitized input and output data could then be used to calculate the linear input and output frequency spectrums using an FFT. The linear spectra were then converted to the power spectra, at which point the coherence and FRF functions could be computed. The conversions from linear to power spectra are defined below. 


$$
\begin{gathered}
X(f): \text { Linear Input Spectrum } \\
Y(f): \text { Linear Output Spectrum } \\
G_{x x}=(X)\left(X^{*}\right): \text { Input Auto Power Spectrum } \\
G_{y y}=(Y)\left(Y^{*}\right): \text { Output Auto Power Spectrum } \\
G_{x y}=(X)\left(Y^{*}\right): \text { Crosspower Spectrum } \\
G_{y x}=(Y)\left(X^{*}\right): \text { Crosspower Spectrum }
\end{gathered}
$$

Finally, the selection of an appropriate FRF estimator is required to produce accurate results. The two primary methods most commonly available in industry are $\mathrm{H}_{1}$ and $\mathrm{H}_{2}$ shown in equations 4.1 and 4.2. The major factor to consider when making the selection is whether there is more likely to be noise generated in the input or output signal. According to Ewins, noise near resonance is likely to influence the force signal, while noise at anti-resonance will affect the response signal(s). $\mathrm{H}_{1}$ will likely be less accurate with noise at the input, while $\mathrm{H}_{2}$ will be more liable to errors with noise at the output (Ewins, 2000).

$$
\begin{aligned}
& H_{1}=\frac{G_{y x}}{G_{x x}} \\
& H_{2}=\frac{G_{y y}}{G_{x y}}
\end{aligned}
$$

More noise was observed near anti-resonances in the FRFs output from the data acquisition system resulting in the $\mathrm{H}_{1}$ estimator being chosen. This decision is further supported by previous work done by Chamberlain et al. and in the referenced text (Ewins, 2000) (Chamberlain, 2018). It should be noted that steps were taken to minimize external vibrations which had the potential to introduce input noise. This was primarily done through the isolation of the vacuum pump used to cool the shaker which was accomplished by moving it from the table on which the shaker was mounted to a thick rubber pad on the floor of the lab.

According to Avitable however, the $\mathrm{H}_{1}$ estimator underpredicts the amplitude of the FRF while the $\mathrm{H}_{2}$ estimator overpredicts the FRF amplitude (Avitable, 2018). It is therefore necessary 
to quantify the level of discrepancy between the two estimators which can be accomplished using the coherence function shown in equation 4.3.

$$
\gamma^{2}=\frac{G_{y x} G_{x y}}{G_{x x} G_{y y}}=\frac{H_{1}}{H_{2}}
$$

The coherence functions are also monitored to ensure the repeatability of the FRF data being collected. A coherence measurement of one would indicate no discrepancy between the two estimators, and serves as a general indicator of high measurement quality. Coherence values will in theory remain between 0 and 1 , and for acceptable measurement should remain between 0.9 and 1 over the frequency range. One exception to this requirement is near anti-resonances, where the output response is very low. At these frequencies the signal to noise ratio is also very low, and it is expected for the coherence to drop (Avitable, 2018). An example of a typical FRF measurement and coherence function is shown in Figure 4.10.
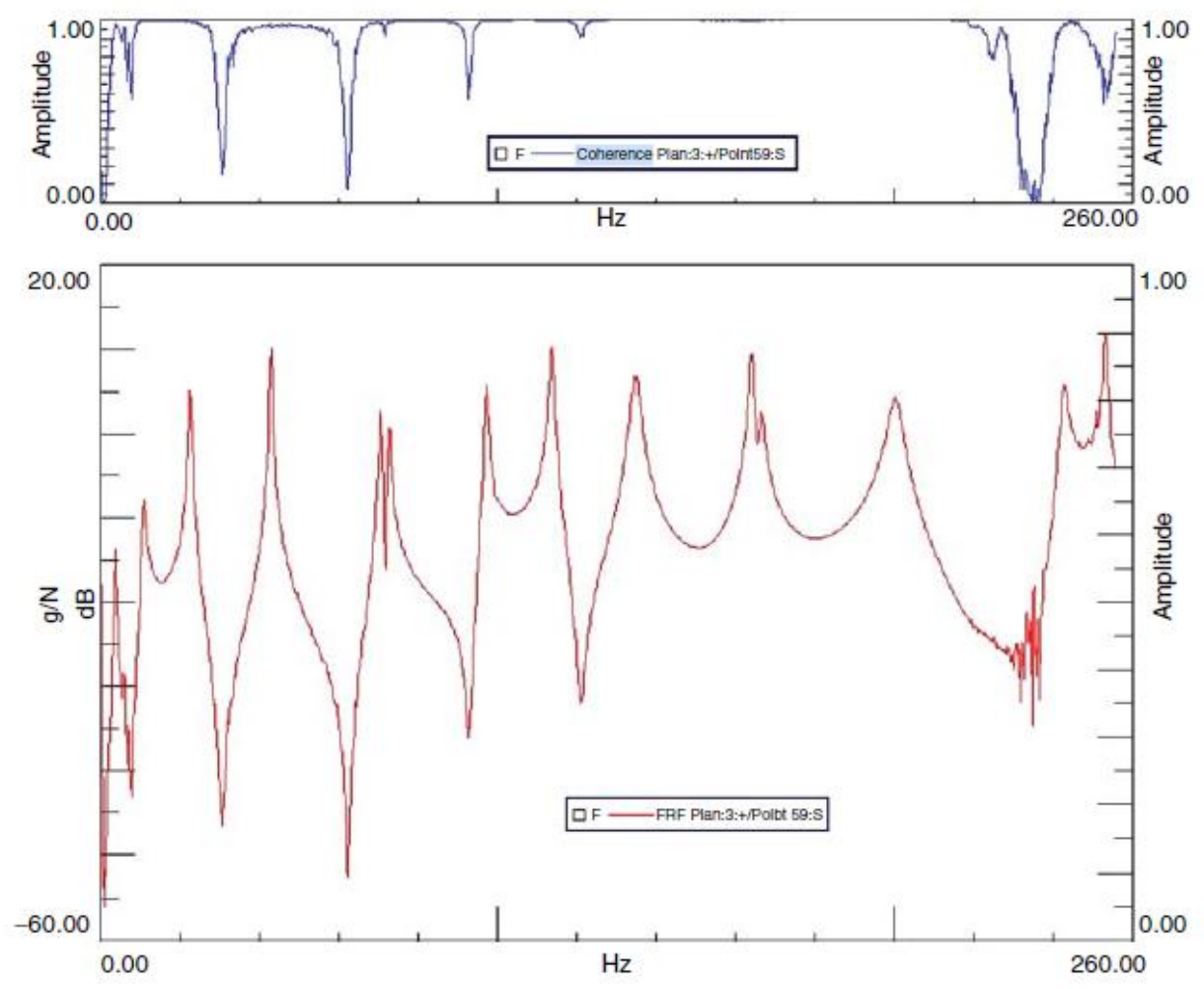

Figure 4.10: Typical FRF and coherence measurement. Notice the dips in coherence in relation to the anti-resonances of the FRF (Avitable, 2018). 


\subsection{Data Analysis}

ME'Scope VES 4000 software was used to extract the modal parameters from the raw data collected during testing. This software provides a curve fitting procedure which applies mathematical expressions to the experimental data, such that natural frequencies, damping values and residues can be extracted. The first step of curve fitting is to identify the position of the resonant peaks in the frequency range of interest. There are two options available to accomplish this: the complex mode indicator function (CMIF) or the multivariate mode indicator function (MMIF). Of these the CMIF proved to be most effective at identifying resonance peaks in the frequency range. The mode indicator function serves two purposes, to provide a single curve for counting the number of resonance peaks, and to limit the frequency and damping curve fitting methods to the data surrounding each resonant peak (Vibrant Technology, 2015).

The next step is to curve fit the data which in this case was done using a global polynomial method. According to Vibrant Technologies, the global polynomial method may be used when the resonant peaks from each DOF show good alignment with respect to frequency, which was found to be the case for the data extracted from both sub-assemblies. This method provides one frequency and one damping value for each resonance in the frequency band which act as global properties for the system (Vibrant Technology, 2015). One factor that allowed this method to be implemented was the use of dummy masses during testing. This ensured that the physical properties of the system remained constant regardless of accelerometer placement, which resulted in closer peak alignment between FRFs. The results from the dummy mass experiment which illustrate their effect on the results are presented in Chapter 5.

The final step is the determination of the residues of each mode in the frequency range. As with curve fitting there are two methods to choose from. According to the Vibrant documentation, the "peak" method is most appropriate for FRF data in which the peaks do not align closely. However, for FRFs which contain closely coupled modes, the peak method is not 
suitable, and a MDOF method which can simultaneously estimate the modal parameters of closely coupled modes should be used. The polynomial method uses all of the real and imaginary data to estimate the numerator polynomial of each FRF using the least squared error curve fitting process (Vibrant Technology, 2015). The denominator is estimated using the frequency and damping terms from each mode found during curve fitting. After the residues are calculated, a shape table containing the frequency and damping terms as global properties, and the magnitude and phase information for each DOF and every mode shape is created. The frequency values are used during the first stage of correlation with the computational model, and the magnitude and phase information is used when correlating mode shapes using the pseudo orthogonality check.

\subsection{Chapter Summary}

A review of the experimental setup including the boundary conditions, excitation methodology and data acquisition parameters was presented in this chapter. Additionally, the techniques used to extract the natural frequencies and mode shapes from the raw data was presented. The sensors used to collect data, and their placement on the structure was also described in detail, in conjunction with the AutoMAC technique used to validate their position during testing. Testing considerations including the driving point location which are specific to the experiments presented were also described for the purposes of progressing this project onto larger structures. In each instance, the choices made were supported by previous work found in the literature, or where applicable, by the technical support resources produced in industry. This chapter aims to facilitate a better understanding of the experimental setup and procedure, as well as the data acquisition and analysis methods. It is now suitable to review the results produced by these experiments and their correlation with the computational model in Chapter 5. 


\section{Chapter 5}

\section{Results and Correlation}

\subsection{Overview}

The following chapter presents the experimental results collected, and their correlation with computational models produced by (Lam \& Mechefske, 2020). The validity checks used to confirm the quality of the experimental results are described, and the results from these checks are discussed. These checks include linearity, repeatability and driving point measurements as per ISO guidelines, as well as additional measurements inspired by the literature. These measurements include a mass effects study and a test to evaluate the effects of stinger length on excitation quality. The results from these validity checks show that the assumptions made regarding linear modal analysis are upheld, however minor issues in the results from these checks were found and are discussed.

The final experimental results including the natural frequencies and mode shapes of both sub-assemblies are presented. Additionally, the techniques used to evaluate the correlation between the experimental and computational results are discussed, and the results of the correlation studies are presented. In general, a strong level of correlation was found between the FEM and experimental natural frequencies and mode shapes for both sub-assemblies. Finally, recommendations are made for both future experimental testing improvements, as well as further model enhancements to improve correlation further.

\subsection{Validity Checks}

\subsubsection{Linearity Check}

One of the primary assumptions associated with the experimental modal analysis techniques implemented, is that the structure behaves in a linear manner. According to Ewins "Most of the theory upon which modal testing and FRF measurement is founded, relies heavily 
on the assumption that the test structure's behavior is linear." (Ewins, 2000). In other words, the response of the structure is linearly related to the force input, and as a result the FRF measurements will remain constant regardless of input amplitude. Additionally, computational modelling performed for this project assumed the structure was linear, further confirming the need for this check. The linearity check evaluates this behavior by repeating a measurement at certain input and output measurement locations at varying amplitude and evaluating the resultant FRF measurements. Non-linear behavior would cause discrepancies in the frequency response plots especially near resonance, which would be exhibited by a change in amplitude of a resonance. A lack of any significant discrepancy in the FRFs produced from this test indicates that the structure is behaving linearly within the range of excitation used.

The procedure used to evaluate response linearity was identical for both sub-assemblies. As stated in Chapter 4, a deterministic signal should be used to evaluate linearity, and as such a sine chirp signal was selected. The literature states that one input-response pair should be used to evaluate linearity, but because eight accelerometers were already connected, eight points were tested on both structures. This was especially important for the second sub-assembly which could potentially exhibit linear behavior in the FESF but non-linear behavior in the RESF due to the amount of mechanical connections between excitation and response measurement locations. It is also recommended that the excitation force be varied by a factor of 10 where possible, however there were limitations in the experimental setup which prevented this from being possible (International Organization for Standardization, 1994). Particularly with the second subassembly, there was a certain threshold excitation below which the entire structure was not properly excited. This is not surprising due to the structure's size, however ten times this threshold excitation would risk damage to the stinger, and potentially the impedance head. For this reason, the excitation signal was only varied by a factor of approximately 6 for the linearity check of the second sub-assembly. 
For the first sub-assembly, four accelerometers were placed on the center bulkhead at points 2, 12, 22 and 42; the schematic for which can be found in Figure 4.7 and four were distributed around the skin at points $72,82,92$ and 112 . The structure was excited through the driving point used for the rest of the experimental testing, and all other factors including the boundary conditions also remained constant. The excitation level was varied from $2 \mathrm{~N}$ to $7 \mathrm{~N}$ then finally to $15 \mathrm{~N}$ peak input. The resultant FRFs produced by the linearity check of sub-assembly one are shown in Figures 5.1 and 5.2. The following figures show the results for only one DOF per graph for ease of viewing, but it should be noted that all DOFs from sub-assembly 1 showed similar results.

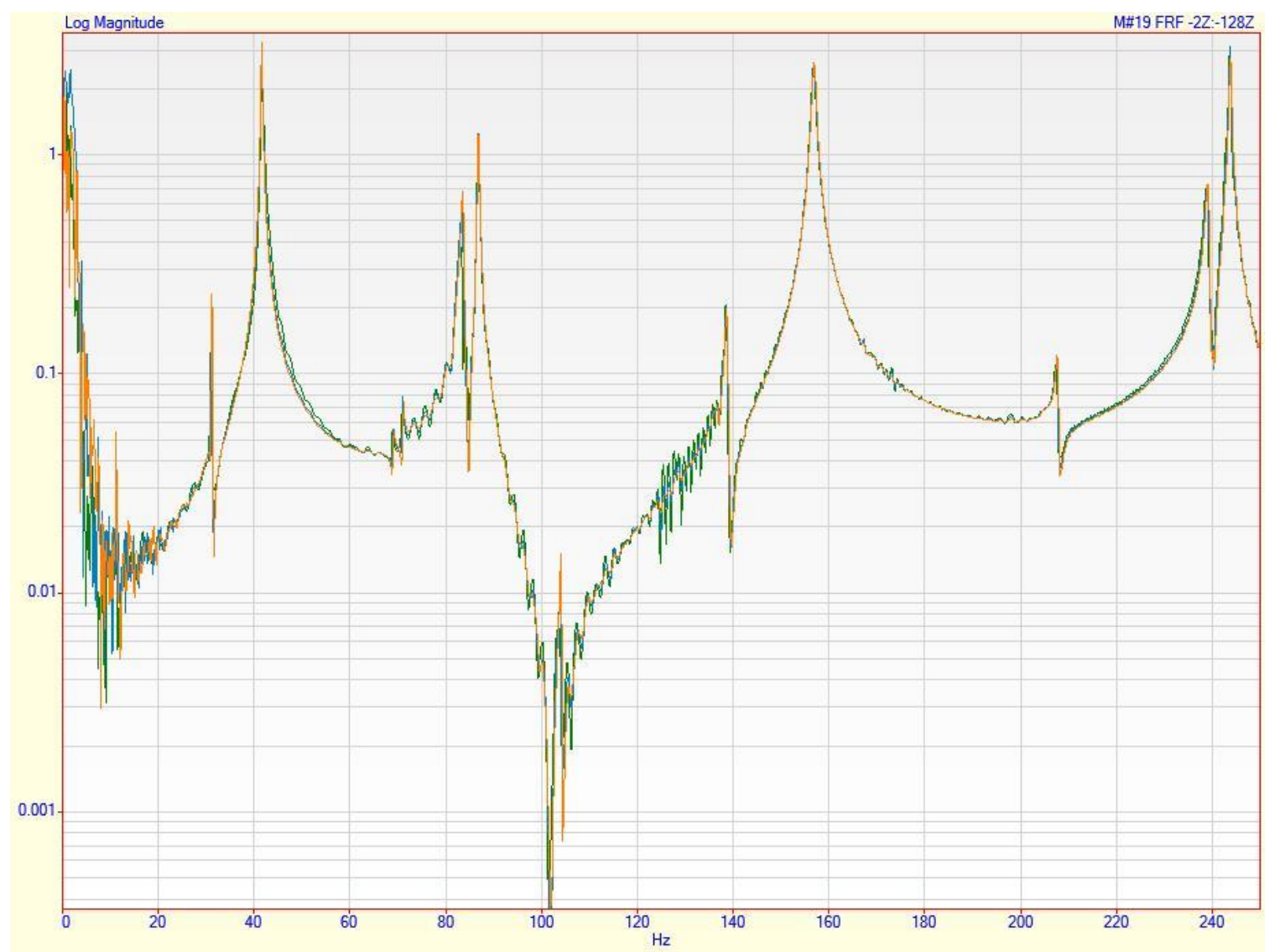

Figure 5.1: Linearity check from DOF 2 on the bulkhead of sub-assembly 1. (Orange 2N, Blue $7 \mathrm{~N}$ and Green $15 \mathrm{~N}$ peak input) 


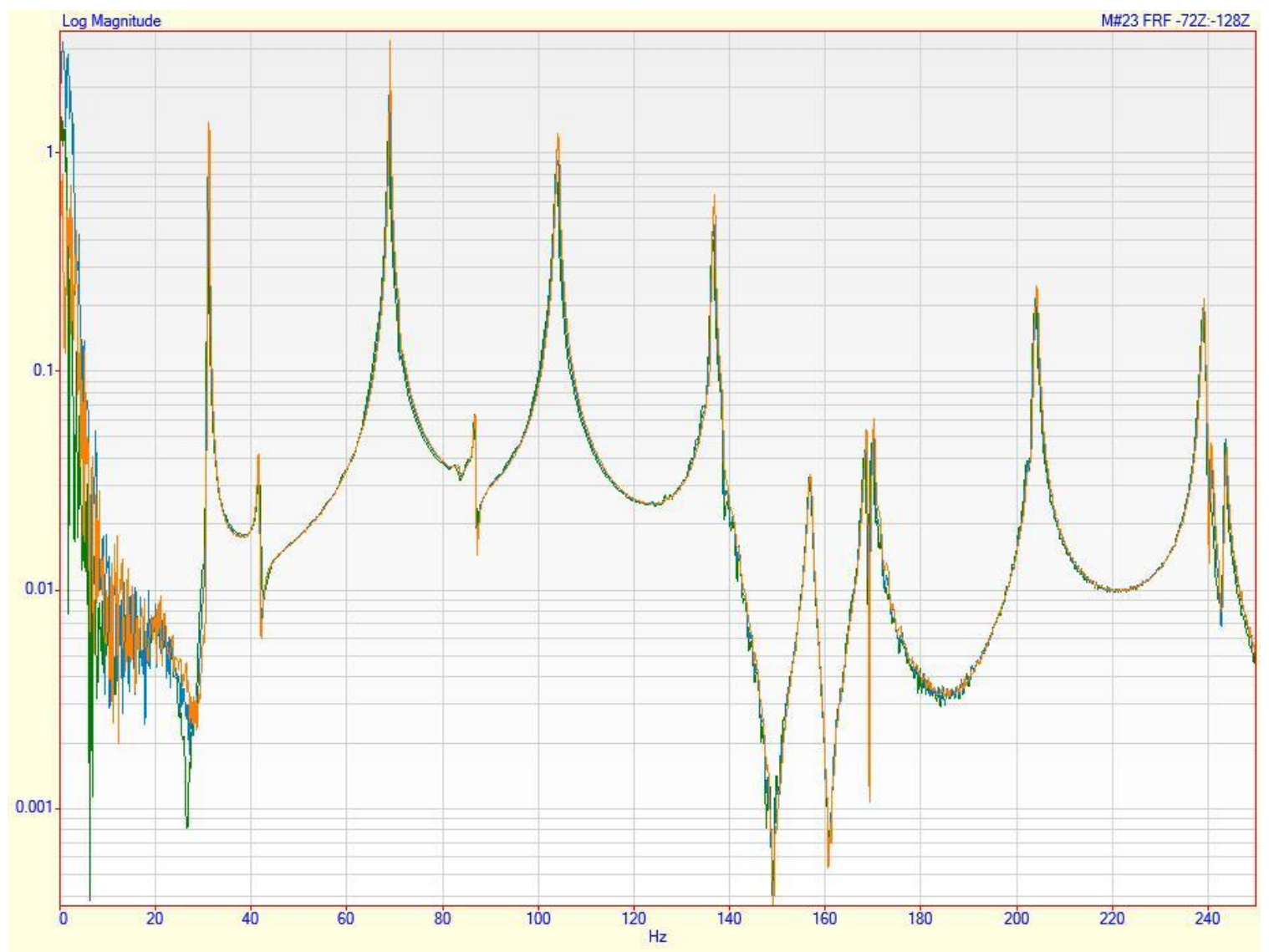

Figure 5.2: Linearity check from DOF 2 on the bulkhead of sub-assembly 1. (Orange 2N, Blue $7 \mathrm{~N}$ and Green $15 \mathrm{~N}$ peak input)

As exhibited by Figures 5.1 and 5.2, the variance of input excitation force from 2 to $15 \mathrm{~N}$ resulted in minimal discrepancies with regards to the resonance peaks. At higher excitation levels, more noise was seen near minima and anti-resonances, which indicates the presence of slight non-linearities. However, due to the consistency of the resonant peak amplitudes, and the elimination of this noise through the implementation of a burst random excitation signal, these noisy regions are not of concern. It can therefore be concluded that sub-assembly 1 shows linear dynamic response, and the assumptions made regarding linear modal analysis are upheld.

To evaluate the linearity of the second sub-assembly, four accelerometers were placed on the FESF at points 4, 40, 44, and 128, and on the RESF at points $64,84,110$, and 144 the schematics for which can be found in Figures 4.8 and 4.9 respectively. Once again 3 excitation 
levels were used: $3 \mathrm{~N}, 10 \mathrm{~N}$ and $20 \mathrm{~N}$ peak input. A sample of the resultant FRFs are shown in

Figures 5.3 and 5.4, and once again only 1 DOF from both the FESF and RESF are showcased for ease of viewing.

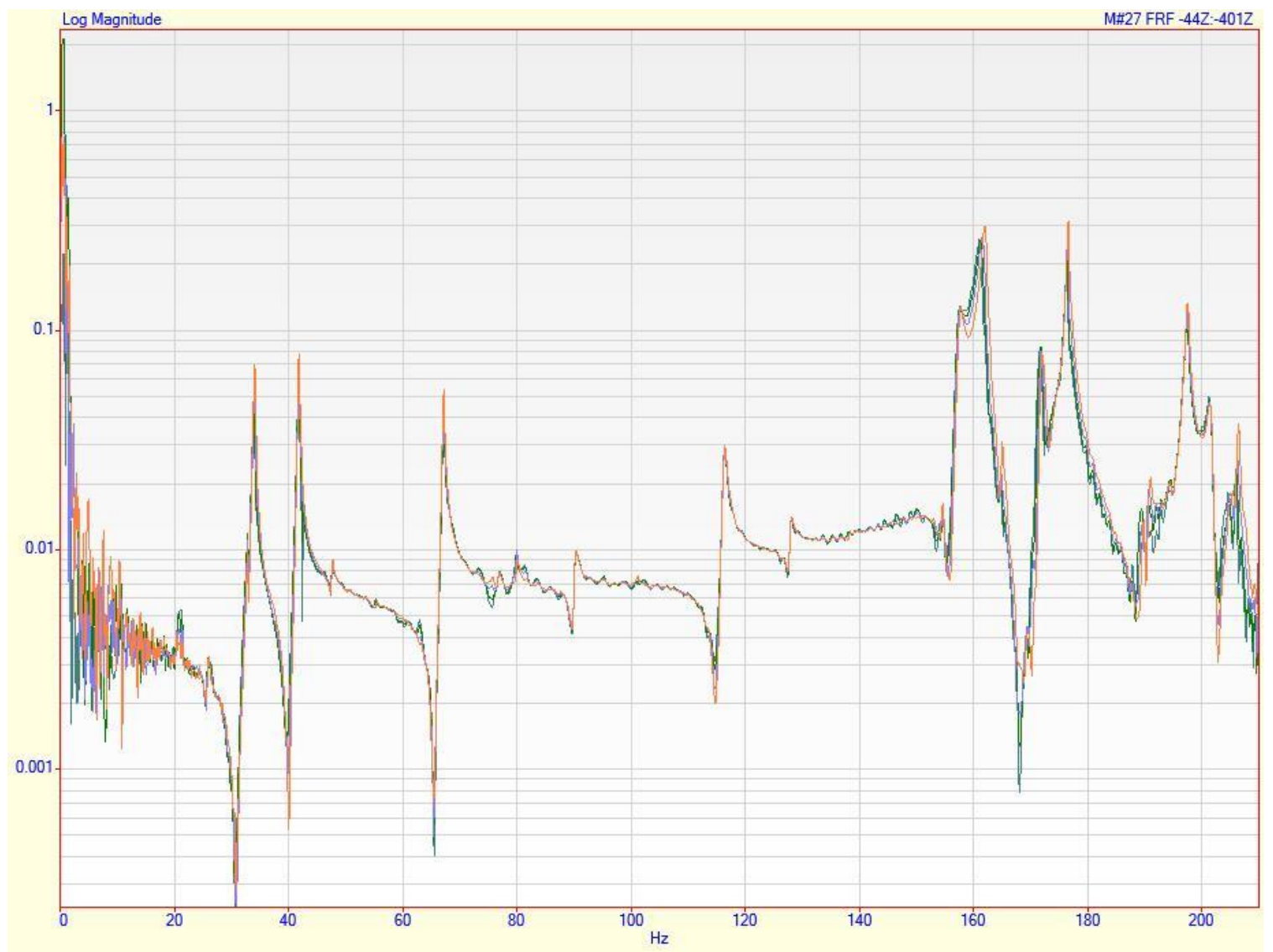

Figure 5.3: Linearity check from DOF 44 on the FESF of sub-assembly 2. (Orange 3N, Blue 10N and Green 20N Peak Input) 


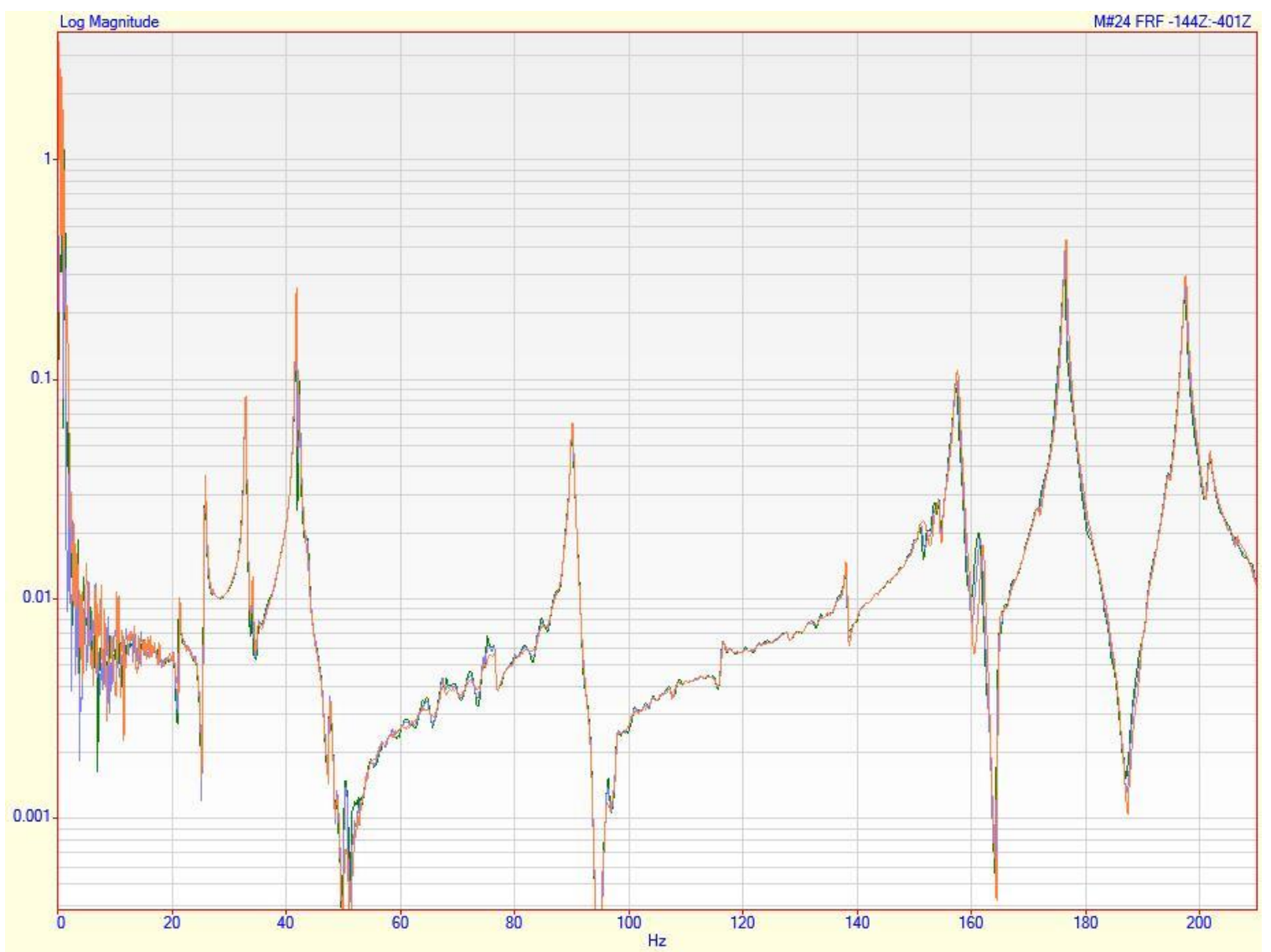

Figure 5.4: Linearity check from DOF 144 on the RESF of sub-assembly 2. (Orange 3N, Blue 10N and Green 20N peak input)

Once again minimal discrepancies are found between the various tests indicating that the structure is behaving in a mostly linear nature. It was found that at higher excitation levels the plots became slightly noisier, as shown by the green and blue traces, however throughout modal parameter extraction, this noise was averaged out using a non-deterministic signal. Once again, the peaks show strong alignment both in frequency and amplitude, and as such, it can be concluded that sub-assembly 2 behaves linearly.

\subsubsection{Repeatability Check}

The repeatability check is used to evaluate the consistency of the experimental setup and procedure, and the reliability of the resultant data. As per ISO guidelines, at least one measurement should be repeated before and after modal parameter extraction (International 
Organization for Standardization, 1994). Multiple points on both sub-assemblies were tested multiple times throughout the testing procedure. In addition to performing the repeatability checks in accordance with the ISO recommendations, measurements were taken each time the sub-assembly was suspended from the support structure. This was done to ensure that any minor change in the supporting boundary conditions did not have a significant effect on the dynamic response of the sub-assembly. For most repeatability checks performed, the data was found to be consistent between trials, and the experimental setup was deemed acceptable. There were some rare instances where discrepancies were noted, however in each case this was found to be a result of setup errors including stinger misalignment or sensor connectivity issues. Once corrections were made, the repeatability check was performed again, and the expected results were found. The following Figures 5.5 and 5.6 show typical results found during the repeatability checks of sub-assembly 1 and 2 respectively.

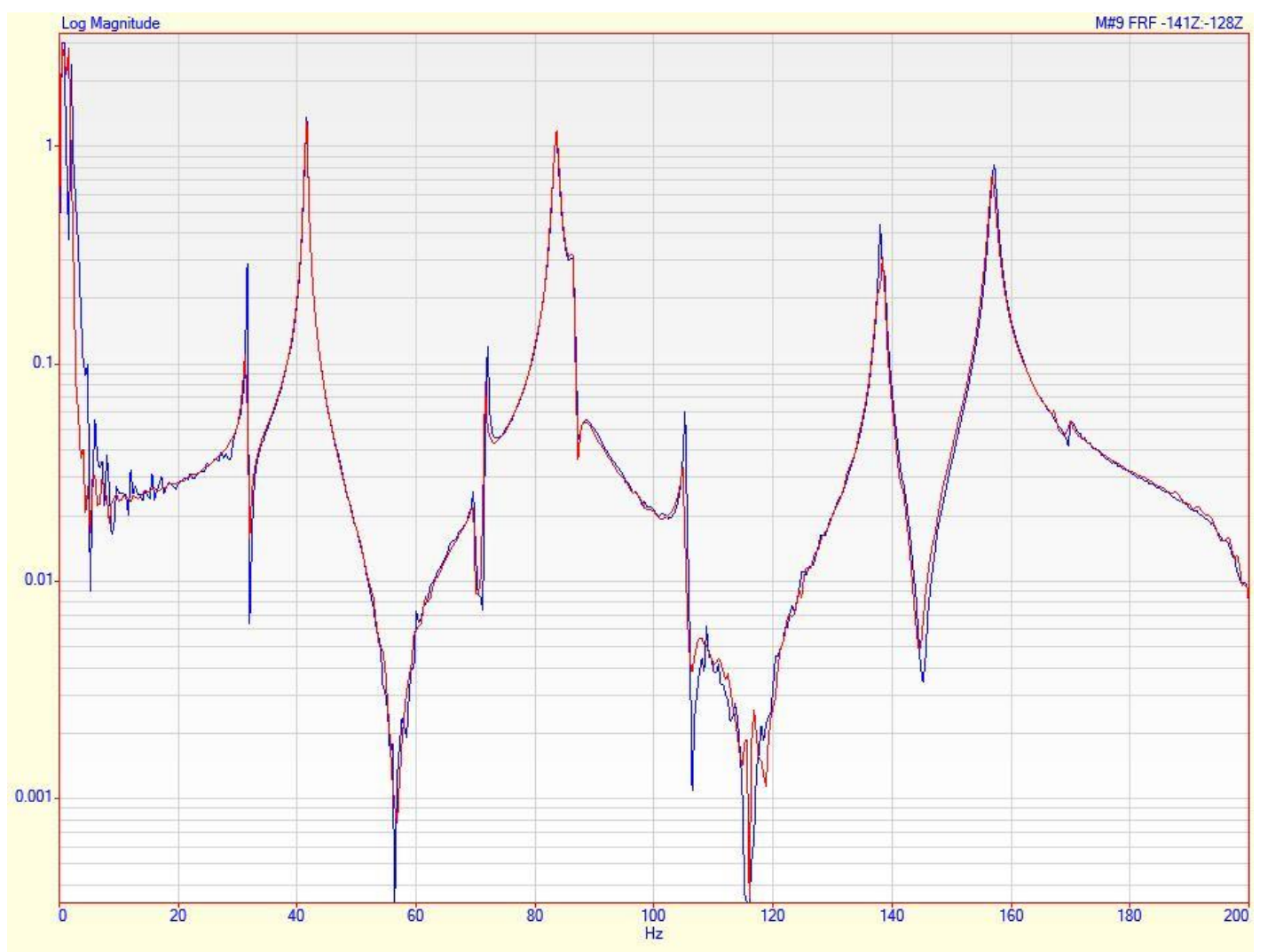

Figure 5.5: Repeatability check of DOF 141 on the bulkhead of sub-assembly 1. 


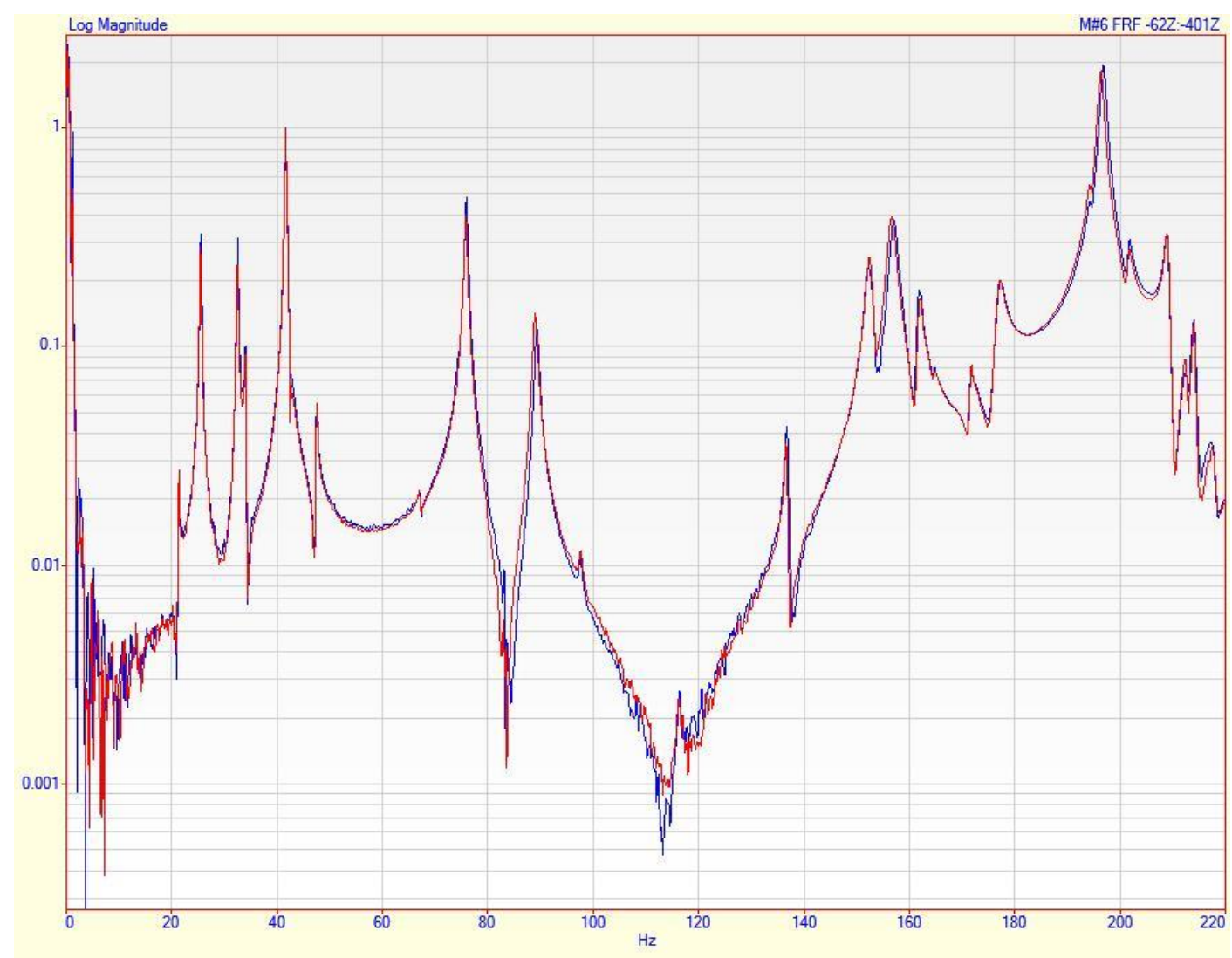

Figure 5.6: Repeatability check of DOF 62 on the RESF of sub-assembly 2.

\subsubsection{Driving Point Measurement}

The driving point measurement is performed by collecting input and output response from the same point on the structure. As described in Chapter 2, the expected result is to see no phase changes in the measured FRF, which can be visualized using two different presentations of the FRF data. Phase changes are associated both with minima in between resonance peaks and with a change from positive to negative (or vice versa) in the imaginary FRF content. When looking at an FRF magnitude plot, it is therefore expected that every resonance peak is followed by an anti-resonance. When looking only at the imaginary content, all resonant peaks should form on one side of the frequency axis. Any deviation from these expected results would indicate an issue with the experimental setup, most likely with the excitation apparatus or transducers. This is most often the case for impact measurements, where the excitation location is slightly variable, 
and thus not perfectly aligned with the measurement location. Using a shaker and impedance head greatly reduces the probability of discrepancies in the driving point measurement, as near perfect alignment is far easier to achieve. The driving point measurements for both subassemblies are presented in Figures 5.7 through 5.10 below. During modal parameter extraction these measurements were checked on multiple occasions, and the expected patterns regarding the anti-resonances in the magnitude plot, and the unidirectional nature of the data in the imaginary domain were found.

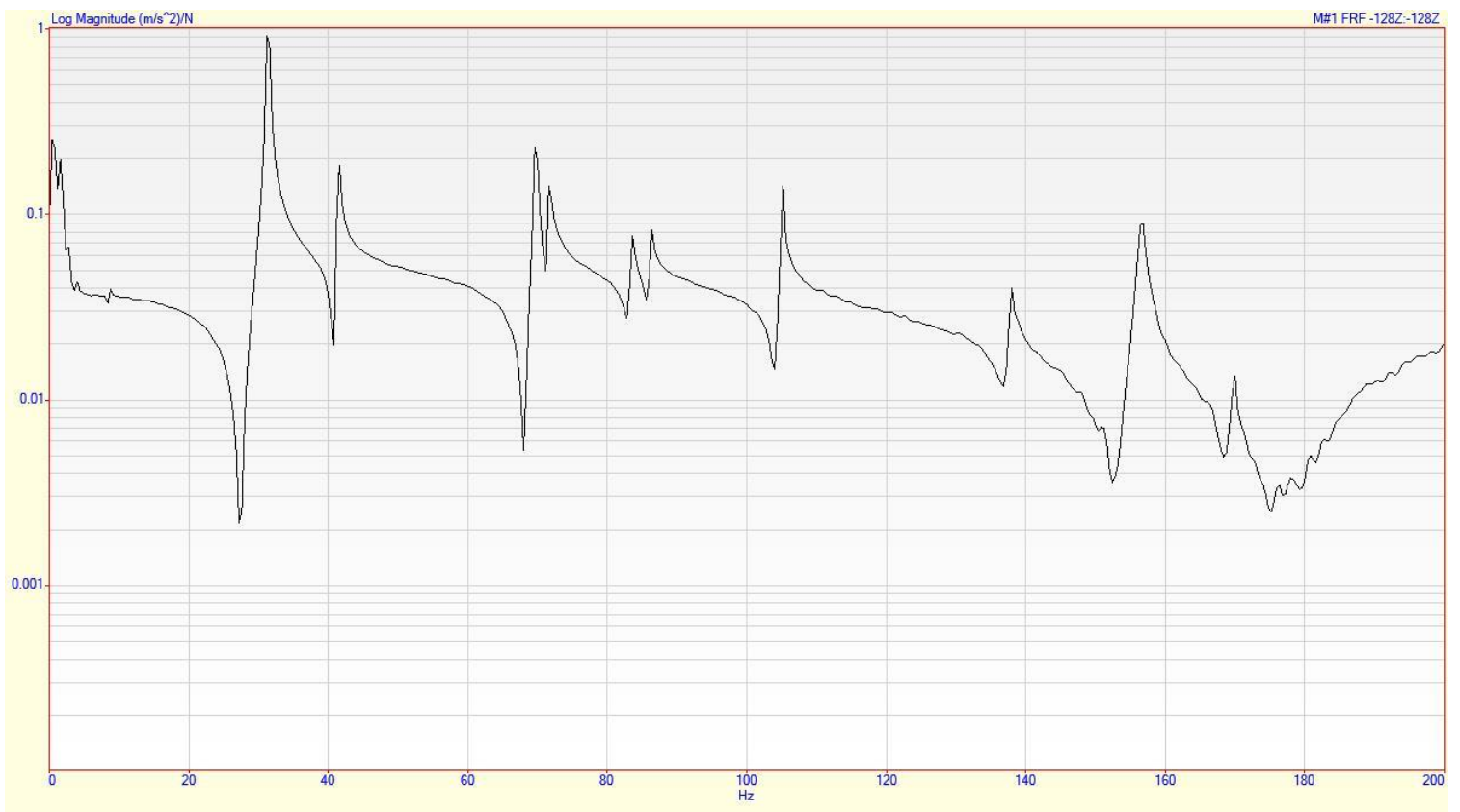

Figure 5.7: Driving point measurement for sub-assembly 1 (Magnitude) 


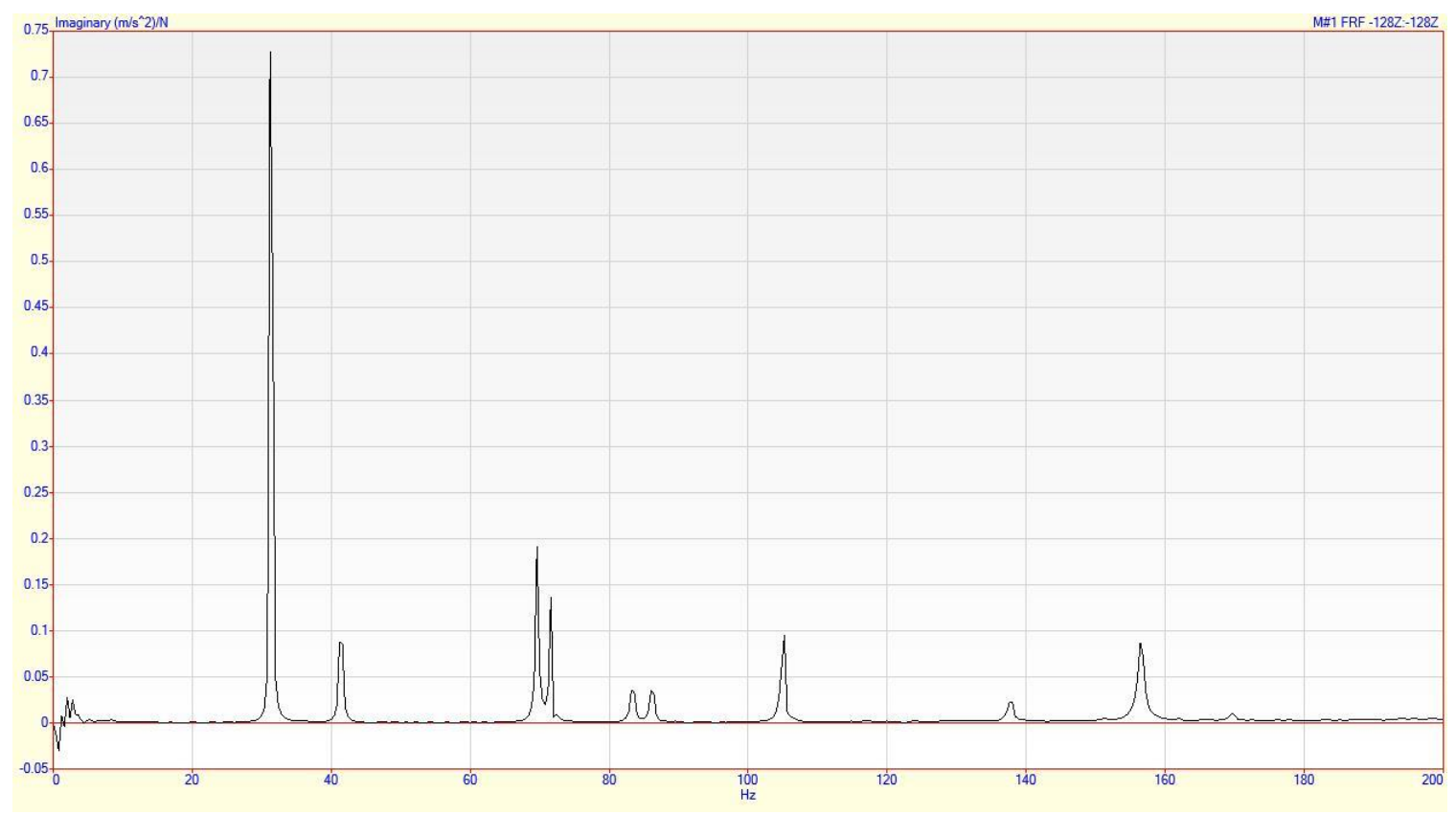

Figure 5.8: Driving point measurement for sub-assembly 1 (Imaginary)

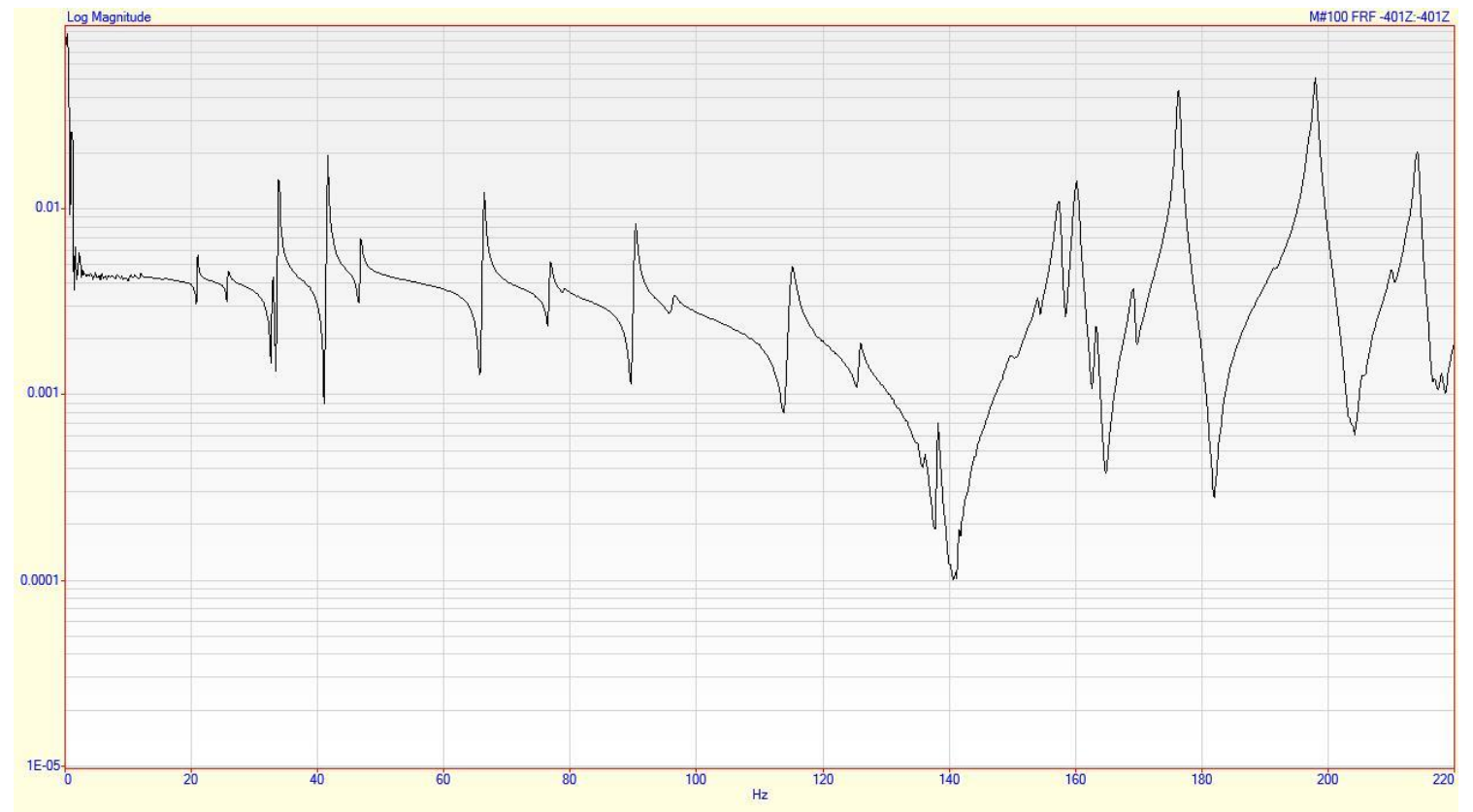

Figure 5.9: Driving point measurement for sub-assembly 2 (Magnitude) 


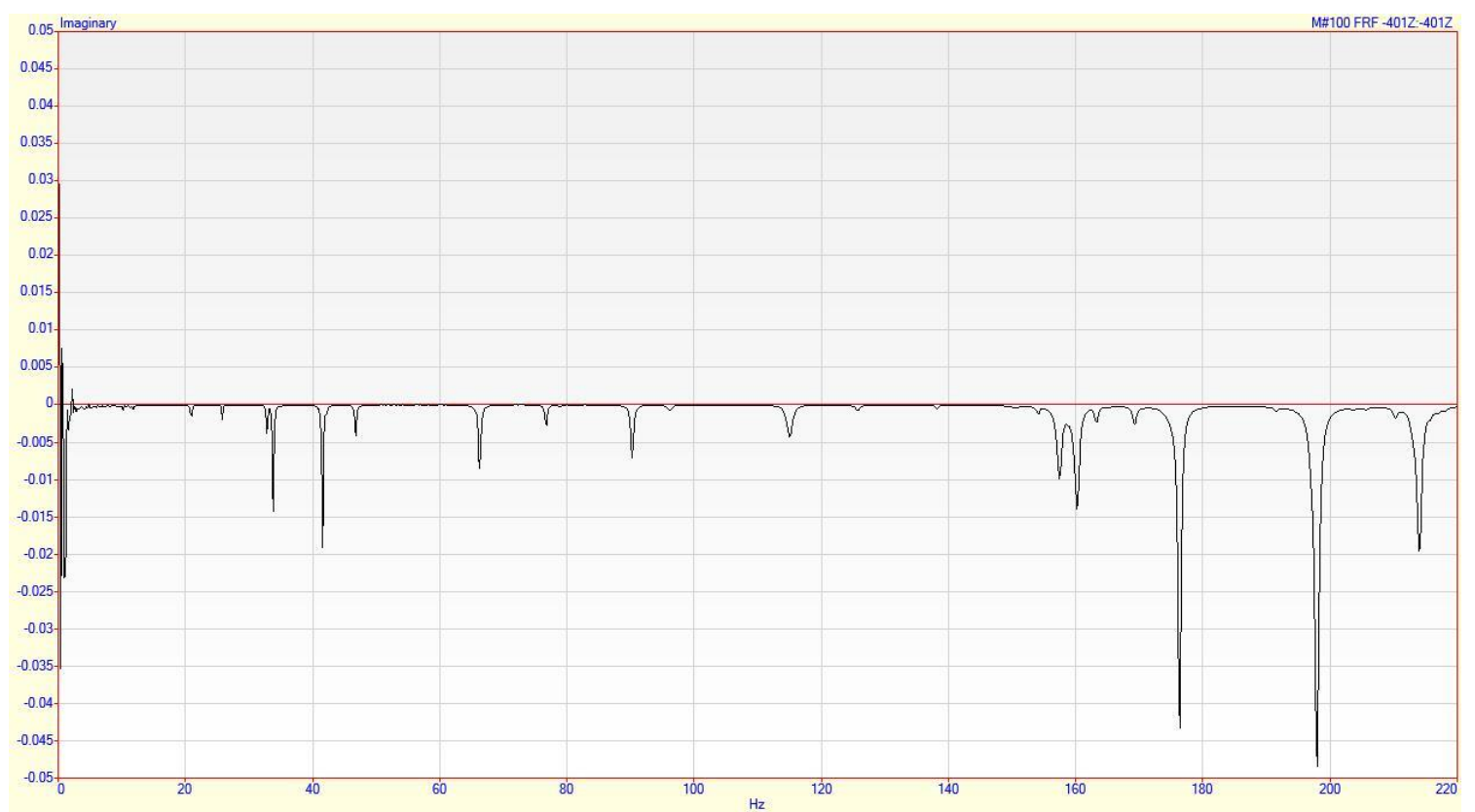

Figure 5.10: Driving point measurement for sub-assembly 2 (Imaginary)

\subsubsection{Mass Effects Study}

To evaluate the effects of the mass imposed by the accelerometers on the dynamic response of each sub-assembly, a mass effects study was performed. The accelerometer mass, while small, can have a significant effect on the natural frequencies of the system. This is especially true when considering the case of eight accelerometers closely grouped during some phases of modal parameter extraction. To obtain a better understanding of the influence of transducer mass on the response of both structures, sub-assembly one was tested under two conditions. First only using accelerometers roving around each DOF, then with the dummy masses in place at each DOF without an accelerometer present. Without dummy masses, the mass distribution of the structure changes depending on the accelerometer placement during any given measurement. This has the potential to effect certain natural frequencies, depending on the mode shapes of the structure, which can lead to misalignment of peaks in the FRF curve. This characteristic was noticed particularly at the resonance at $160 \mathrm{~Hz}$ where a frequency variance of 3 
$\mathrm{Hz}$ was found. Figure 5.11 illustrates the FRF curves from the bulkhead of sub-assembly one overlayed to highlight this misalignment.

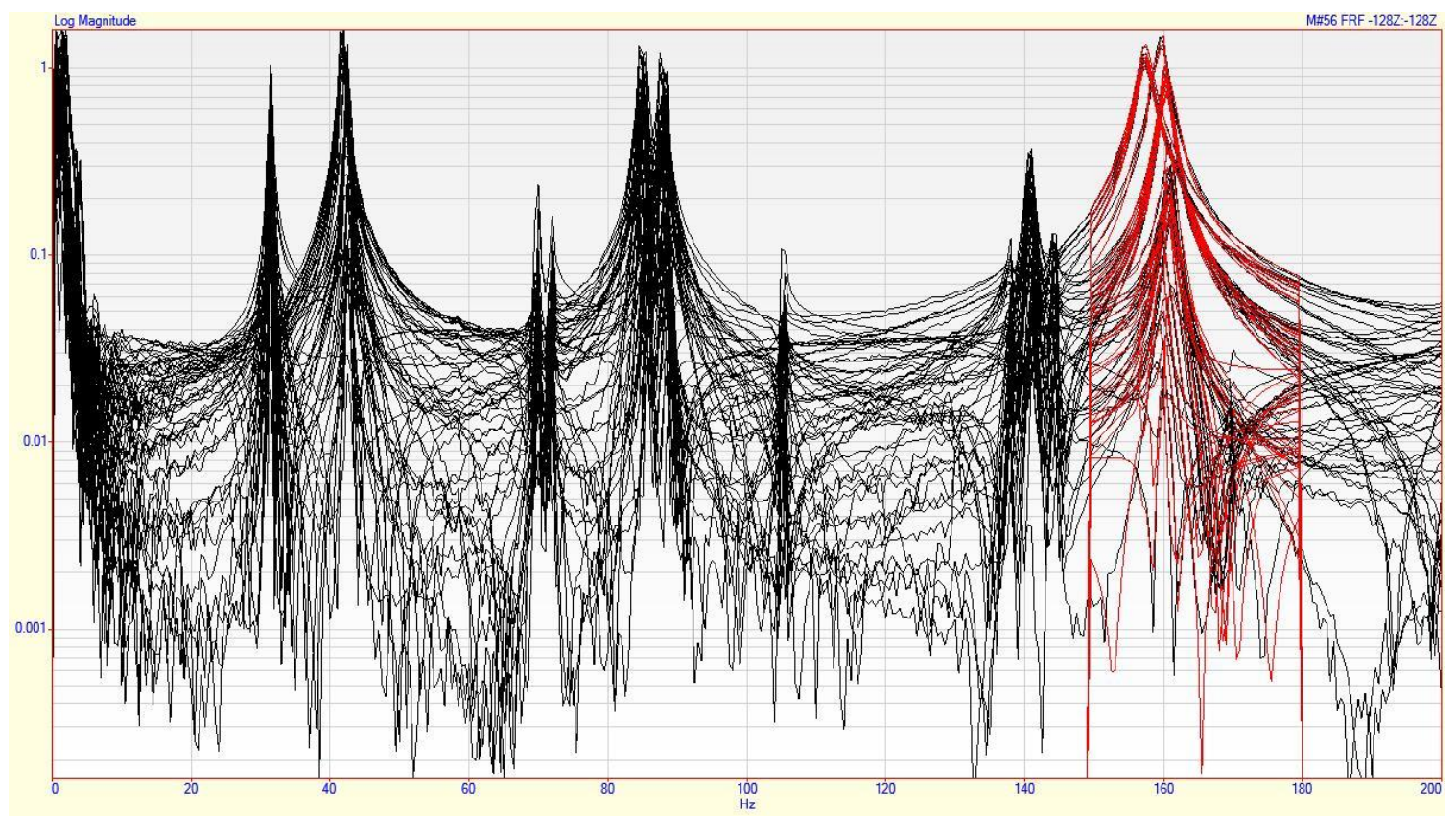

Figure 5.11: Overlayed FRF curves for bulkhead of sub-assembly 1 (No Dummy Masses)

Dummy masses were then added to the structure and the data was collected again. This time, a noticeable improvement with regards to the alignment of FRF peaks was observed and is shown in Figure 5.12. The most severe misalignment was again seen on the peak near $160 \mathrm{~Hz}$; however, it was reduced from $3 \mathrm{~Hz}$ to $1.4 \mathrm{~Hz}$. The other resonances in the frequency range also showed closer alignment, further emphasizing the benefits of using dummy masses in subsequent experiments. It should also be noted that each natural frequency was reduced by approximately 1 $\mathrm{Hz}$, which was not a surprising result given the additional system mass. This added mass was accounted for in the FEM, and as such was not a concern during correlation between experimental and computational results. If a more precise modal data is required from structures with closely grouped DOF locations, then non-contact measurement devices such as laser vibrometers should be considered. 


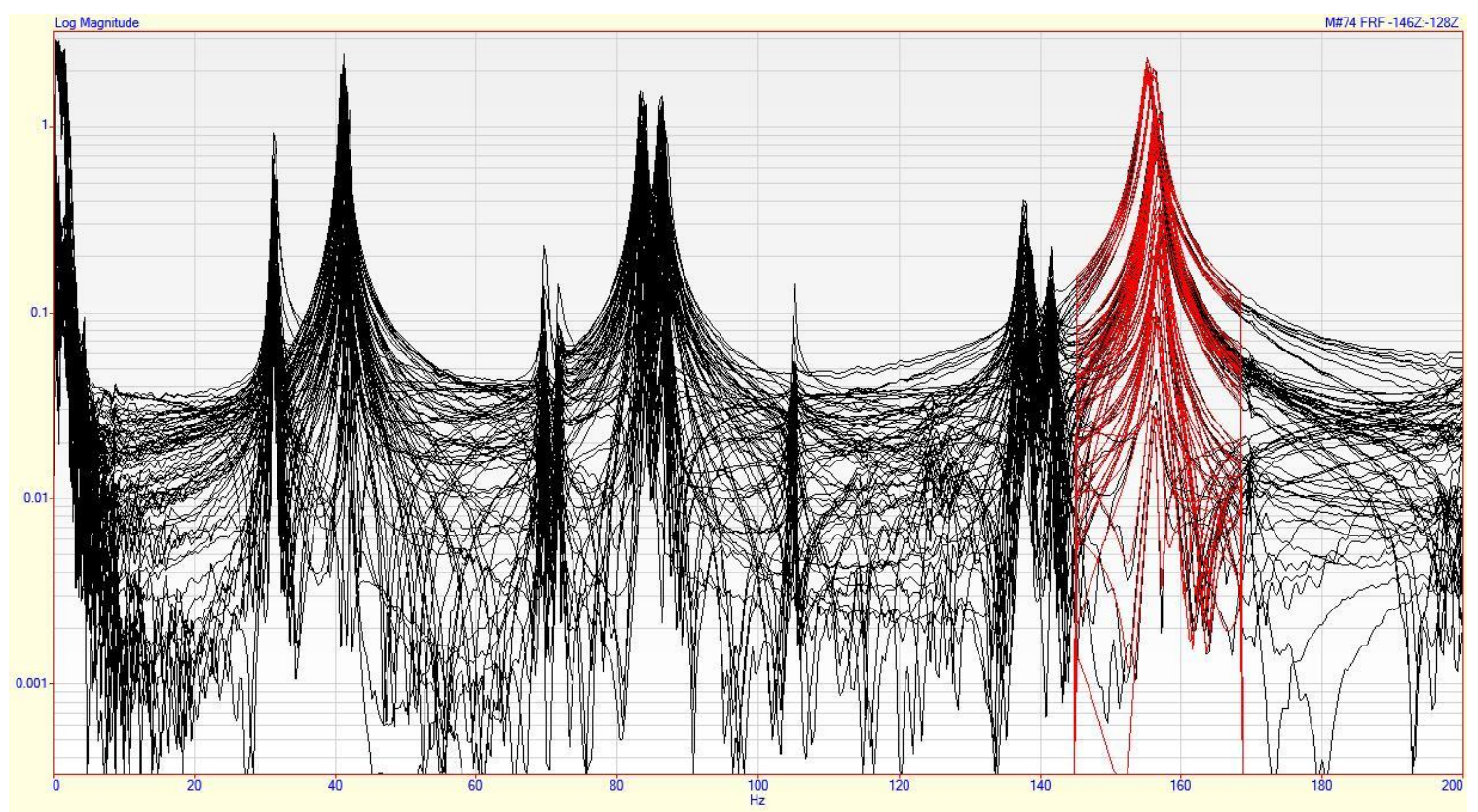

Figure 5.12: Overlayed FRF curves for bulkhead of sub-assembly 1 (With Dummy Masses)

\subsubsection{Response Measurement Placement Validation}

The preliminary DOF placement for both structures was guided by the predicted mode shapes determined using the computational model. There is a certain level of trial and error associated with this process, so a method of quantifying the quality of results produced by a certain DOF layout was required. The MAC provides an indication of eigenvector orthogonality, and therefore reveals the confidence with which mode shapes can be differentiated. The POC is often regarded as the most rigorous correlation method, however an AutoMAC computation function was integrated into ME'Scope. This allowed for DOF layouts to be evaluated more quickly, with less data conversions and transfers required, so the AutoMAC was selected at this stage. An auto orthogonality matrix was produced in conjunction with the POC used to evaluate mode shape correlation later in the experiment, which acted as further validation for the final DOF placement. Figures 5.13 and 5.14 below show the AutoMAC results for sub-assembly one before and after points 129-146 were added to the aft bulkhead of sub-assembly 1 . Figure 5.15 shows the AutoMAC results for sub-assembly 2. Additional experiments were performed with 
additional DOF locations on both the FESF and RESF, however negligible improvements were observed, so the initial placement of DOF locations was used for subsequent experiments. The higher diagonal terms found in the first four modes of the FESF and the first three modes of the RESF will be explained when discussing mode shape correlation in section 5.7.
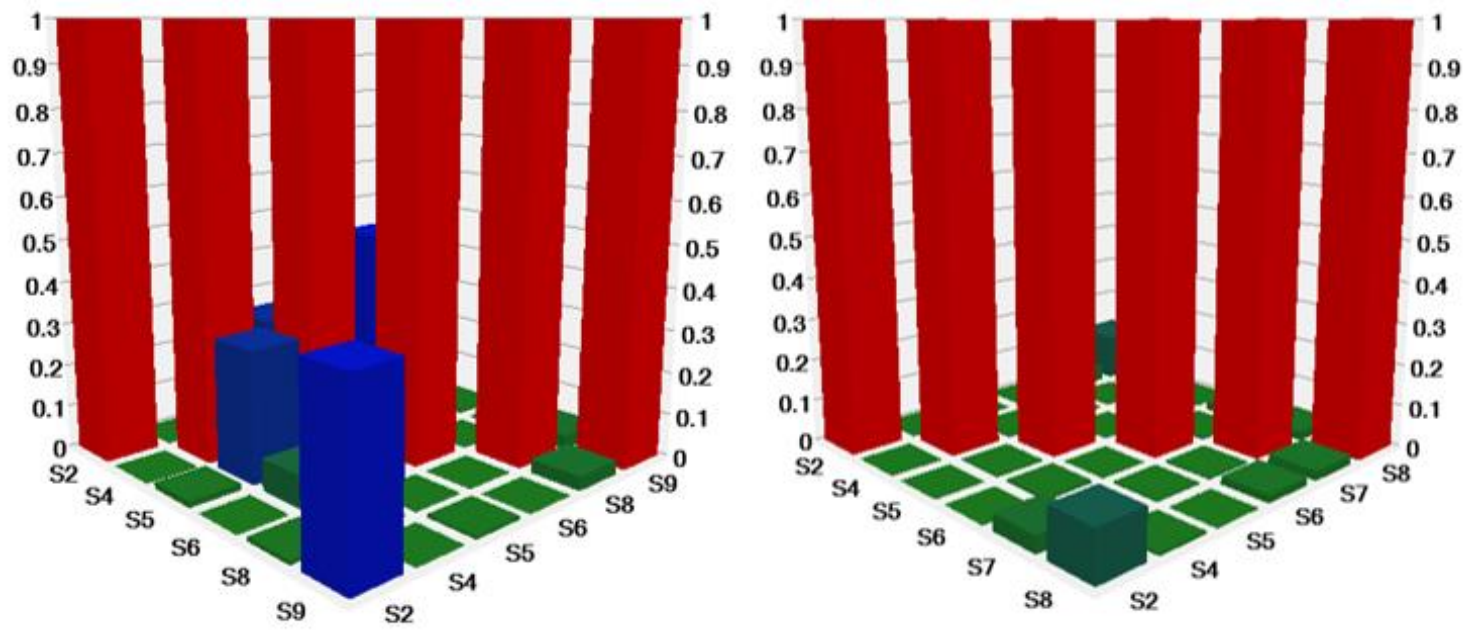

Figure 5.13: Aft Bulkhead AutoMAC before (Left) and after (Right) points were added.

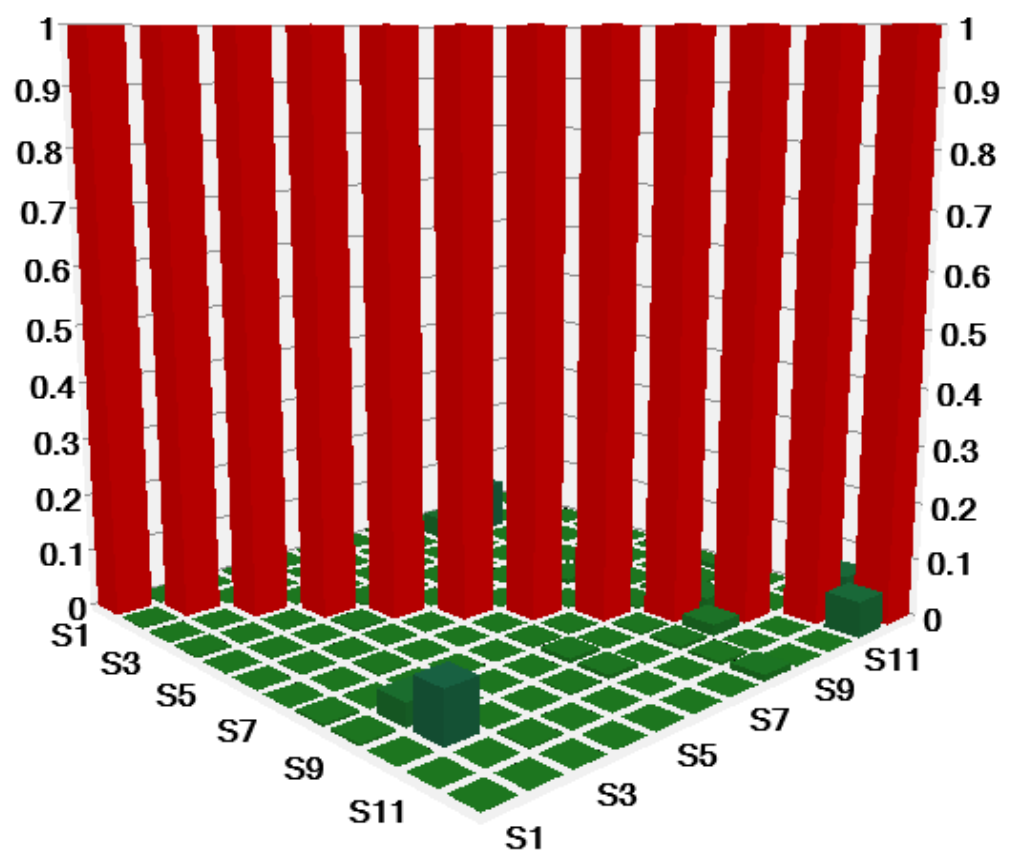

Figure 5.14: Sub-assembly 1 AutoMac for all modes after points were added. 

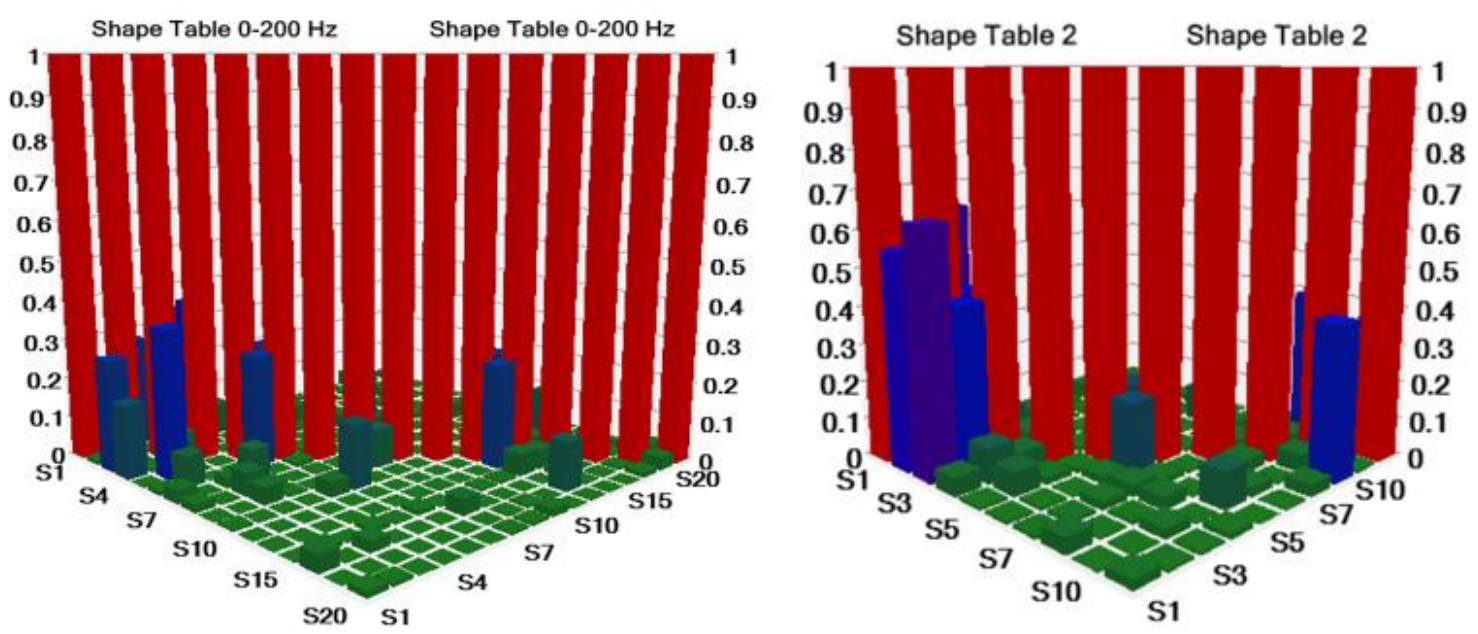

Figure 5.15: Sub-assembly 2 AutoMAC for the FESF (Left) and RESF (Right)

\subsubsection{Stinger Length Test}

The final check performed prior to modal parameter extraction evaluated the effects of stinger length on the experimental results. There are two primary concerns associated with stinger length. If the stinger is to short, lateral stiffness could be imposed on the structure, thus contaminating results, and a stinger which is too long could introduce additional peaks into the FRF curves caused by stinger resonance (Peres, Bono, \& Avitable, 2012). Once again, subassembly one was used in conjunction with 8 accelerometers distributed around the structure. As with previous validity checks, four accelerometers were placed on the center bulkhead, and four were placed on the skin. Three measurements were performed using a similar procedure to that used for the repeatability check, except between trials, the stinger length was varied. Three different stinger lengths were used ranging from $10.5 \mathrm{~cm}$ to $15 \mathrm{~cm}$ and finally $25 \mathrm{~cm}$.

Unfortunately, due to geometric limitations with the skin overhanging from both sub-assemblies, it was not possible to test shorter stingers. The results from this test are presented in Figure 5.16 which shows an overlaid plot of the three FRFs from DOF 42 of the first sub-assembly. Over the $200 \mathrm{~Hz}$ frequency range, negligible discrepancies are found both at resonance and lower amplitudes. The longest stinger did produce slightly noisier results, so the use of shorter stingers 
over this frequency range is recommended for future testing. It can be concluded however, that for the experiments presented, the length of the stinger is shown to have little effect on the natural frequencies or mode shapes. Moving forward a stinger length of approximately $11 \mathrm{~cm}$ was used during the modal parameter extraction of both sub-assemblies.

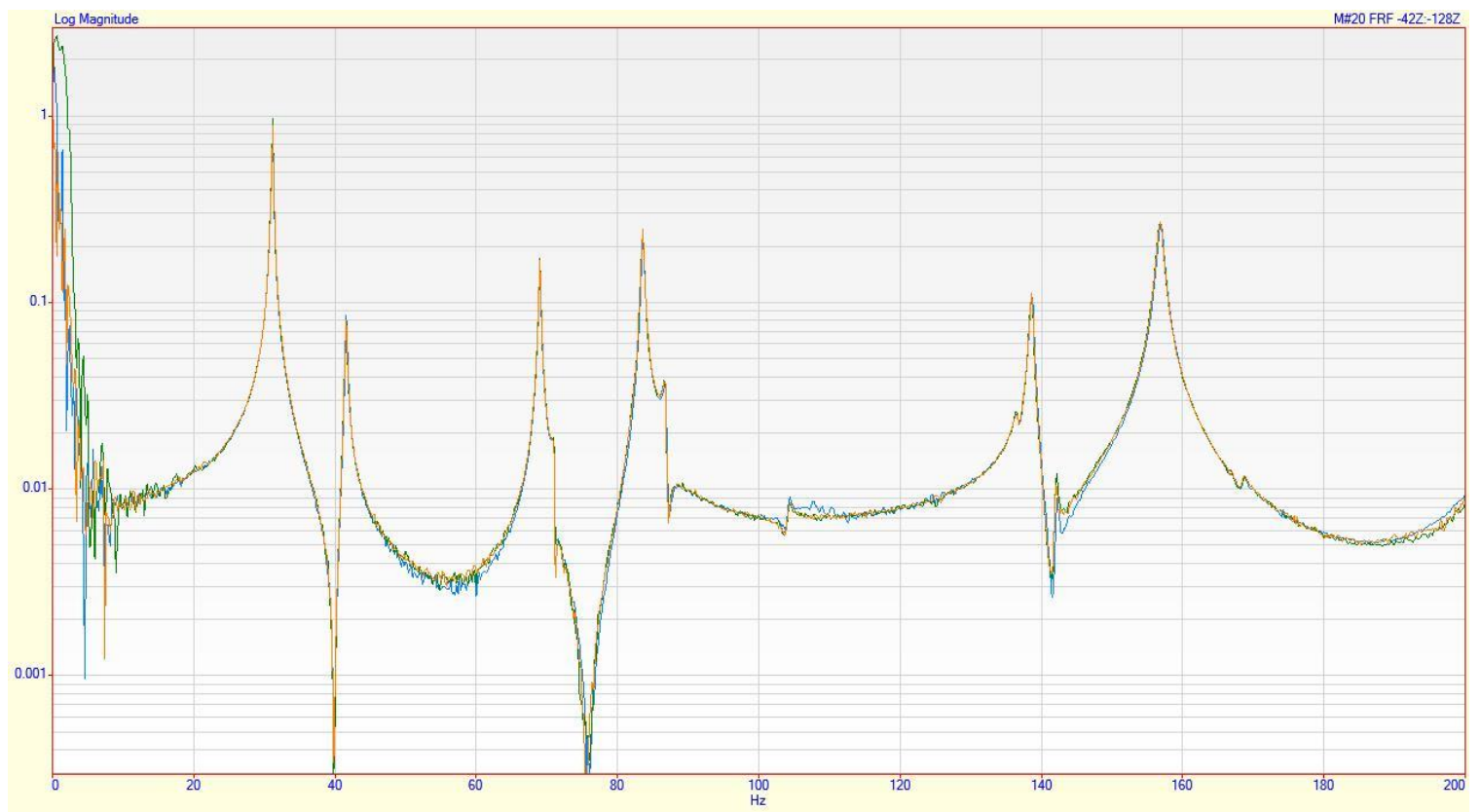

Figure 5.16: Stinger length comparison for DOF 42 of sub-assembly 1. (Orange: $10.5 \mathrm{~cm}$, Green: $15 \mathrm{~cm}$, Blue: $25 \mathrm{~cm}$ )

\subsection{Correlation Overview}

As a result of the numerous validity checks implemented prior to testing, there is now an acceptable level of confidence in the experimental results collected. The experimental data collected included the natural frequencies and mode shapes, which were then compared with the results produced computationally. Where applicable, recommendations were made regarding future modelling improvements, and in the case of the first sub-assembly, a significant improvement in natural frequency correlation was produced. The methods used to compare mode shapes including both visual and mathematical techniques are also discussed. In some instances, the results indicated that more DOFs were required, which was found to be the case for the bulkhead of sub-assembly one. Once the necessary improvements to both the experimental setup 
and computational model were made, a strong level of correlation was found between the sets of data.

\subsection{Sub-assembly One Natural Frequency Correlation}

Fourteen mode shapes were predicted computationally, and thirteen modes were found experimentally in the $200 \mathrm{~Hz}$ bandwidth and visually correlated. The natural frequencies between the two sets were compared as shown in Table 5.1. A skin mode and bulkhead mode (highlighted in yellow) were too closely coupled to separate. This meant that correlation with the computational model for these modes was not possible. Highlighted in green are the mode shapes found in the skin sections of sub-assembly one, while the rows highlighted in blue are the bulkhead modes. When evaluating the percentage difference between the preliminary model and experimental natural frequencies, a significant discrepancy between the natural frequencies related to the skin modes of the structure was observed. It was theorized that by bending each skin section around the center frame, a pre-stress was imposed effectively stiffening the skin. As

a result, the initial computational model underpredicts the skin mode natural frequencies by 10-14 \%. A significant amount of work was performed by (Lam \& Mechefske, 2020) to evaluate the most accurate way to stiffen the skin in the computational model. Multiple updating techniques were evaluated to find a technique that added stiffness to the skin without contaminating the bulkhead results or mode shape data. The most successful methodology involved increasing the modulus of elasticity of the stringers by $100 \%$. This technique along with many other trialed are described in detail by (Lam \& Mechefske, 2020). The final model was able to predict natural frequencies within $6 \%$ and it can therefore be concluded that the updated model of sub-assembly one is successfully predicting natural frequency. 
Table 5.1: Comparison of sub-assembly 1 natural frequencies (Skin modes in orange, bulkhead modes in blue)

\begin{tabular}{|c|c|c|c|c|c|c|}
\hline Mode Description & CMP & $\begin{array}{c}\text { Experimental } \\
\mathbf{( H z )}\end{array}$ & $\begin{array}{c}\text { Preliminary } \\
\text { Model (Hz) }\end{array}$ & $\begin{array}{c}\text { Preliminary Model } \\
\text { Discrepancy (\%) }\end{array}$ & $\begin{array}{c}\text { Revised } \\
\text { Model } \\
\text { (Hz) }\end{array}$ & $\begin{array}{c}\text { Final Model } \\
\text { Discrepancy (\%) }\end{array}$ \\
\hline Oval Skin 2 & 1 & 31.3 & 27.2 & -13.22 & 30.84 & -3.2 \\
\hline Bulkhead Drum & 2 & 41.1 & 43.6 & 6.00 & 43.55 & 5.9 \\
\hline Triangle Skin & 3 & 69.7 & 61.4 & -11.89 & 70.05 & 0.5 \\
\hline Triangle Skin 2 & 4 & 71.6 & 62.4 & -12.90 & 71.35 & -0.4 \\
\hline Bulkhead Halves & 5 & 83.3 & 86.5 & 3.83 & 86.59 & 3.9 \\
\hline Bulkhead Halves 2 & 6 & 86.2 & 87.8 & 1.84 & 87.82 & 1.9 \\
\hline Square Skin 1 & 7 & 105 & 93.0 & -11.39 & 106.32 & 1.3 \\
\hline Five Point Skin & & 138 & & & 140.35 & 1.7 \\
\hline Bulkhead Quarters & & 138 & 142.1 & 0.07 & 142.24 & 1.5 \\
\hline $\begin{array}{c}\text { Bulkhead Quarters } \\
\text { 2 }\end{array}$ & 8 & 142 & 142.00 & 162.67 & 4.9 \\
\hline $\begin{array}{c}\text { Bulkhead Double } \\
\text { Drum }\end{array}$ & 9 & 155 & 162.6 & 4.87 & 172.78 & 1.6 \\
\hline 6 Point Star Skin & 10 & 170 & 152.9 & -10.07 & 174.07 & 1.2 \\
\hline 6 Point Star Skin 2 & 11 & 172 & 153. & -10.95 & \\
\hline
\end{tabular}

\subsection{Sub-assembly One Mode Shape Correlation}

Of the fourteen predicted mode shapes, thirteen were found experimentally and visually correlated, and eleven were mathematically correlated. It was found experimentally that a skin mode and bulkhead mode both resonated at $138 \mathrm{~Hz}$. The computational model predicted a slight separation in natural frequency between these mode shapes and as a result, these two mode shapes could not be mathematically related. Additionally, experimental differentiation of one mode was not possible due to the closely coupled nature of axisymmetric modes. As stated by Ewins, axisymmetric structures can often undergo dynamic behavior resulting in repeated modes as illustrated in Figure 5.17. For perfectly symmetric structures at a given natural frequency, one mode will be found as a combination of all possible resonant shapes (Ewins, 2000). In reality, the first sub-assembly is only nearly axisymmetric, resulting in multiple closely coupled modes. In the case of the "square skin" modes, the two mode shapes predicted by the model were too closely coupled to separate in the experimental data. As a result, the second "square skin" mode shape was also not correlated with the model. The nearly axisymmetric property of sub-assembly 
1 in addition to the closely coupled bulkhead and skin mode discussed earlier, meant that only 11 of 14 predicted modes could be correlated using the following techniques.

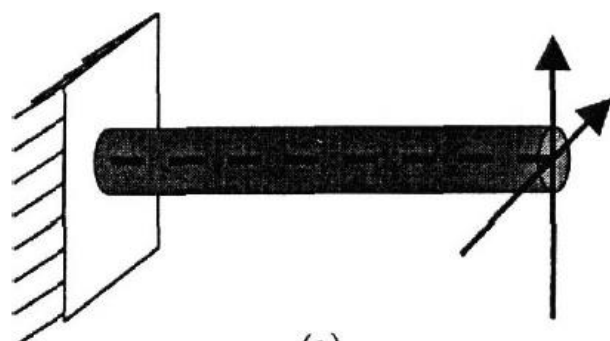

(a)

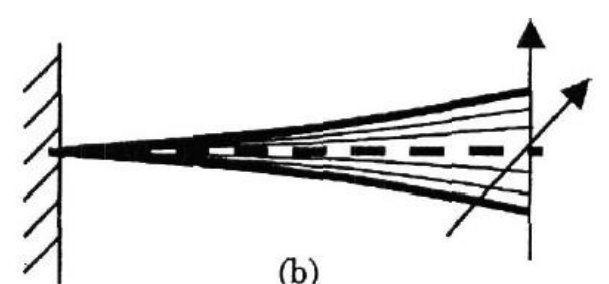

(b)

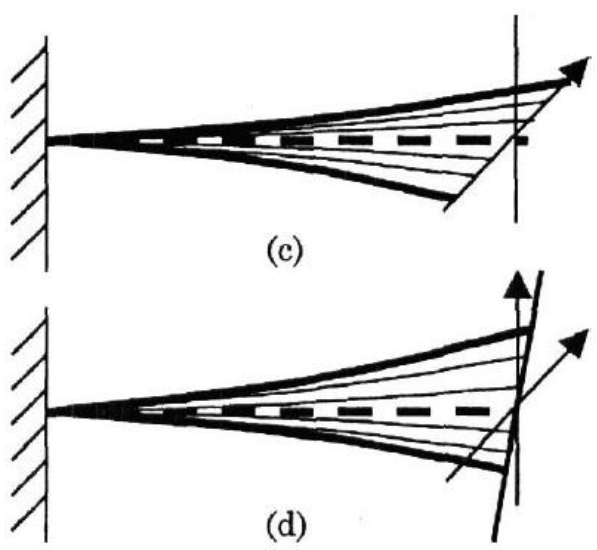

Figure 5.17: Repeated modes of axisymmetric structures (Ewins, 2000).

The first stage of mode shape correlation involves performing a qualitative evaluation of the shapes found experimentally, with those predicted computationally. To produce a graphical representation of each experimental mode shape, ME'Scope uses the amplitude and phase data extracted experimentally from each DOF to generate animations of the structure at resonant frequencies. These were then compared with the graphical mode shape output from the FEM, at which point a list of correlated mode pairs was generated. Thirteen of the fourteen predicted modes were visually correlated, and an example of this visual correlation is shown in Figures 5.18 and 5.19. The overlapping experimental modes at $138 \mathrm{~Hz}$ and their computational counterparts are shown in Figures 5.20 and 5.21 to illustrate the issue which presented itself while trying to mathematically correlate modes. 
Contour Plot

Eigen Mode(Mag)

Analysis system

$1.135 \mathrm{E}+00$
$-1.009 \mathrm{E}+00$
$-8.829 \mathrm{E}-01$
$-7.568 \mathrm{E}-01$
$-6.307 \mathrm{E}-01$
$-5.046 \mathrm{E}-01$
$-3.784 \mathrm{E}-01$
$-2.523 \mathrm{E}-01$
$-1.262 \mathrm{E}-01$
$-7.329 \mathrm{E}-05$

- No result

Max $=1.135 \mathrm{E}+00$

Grids 19321

Min $=7.329 \mathrm{E}-05$

Grids 5293

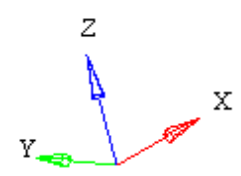

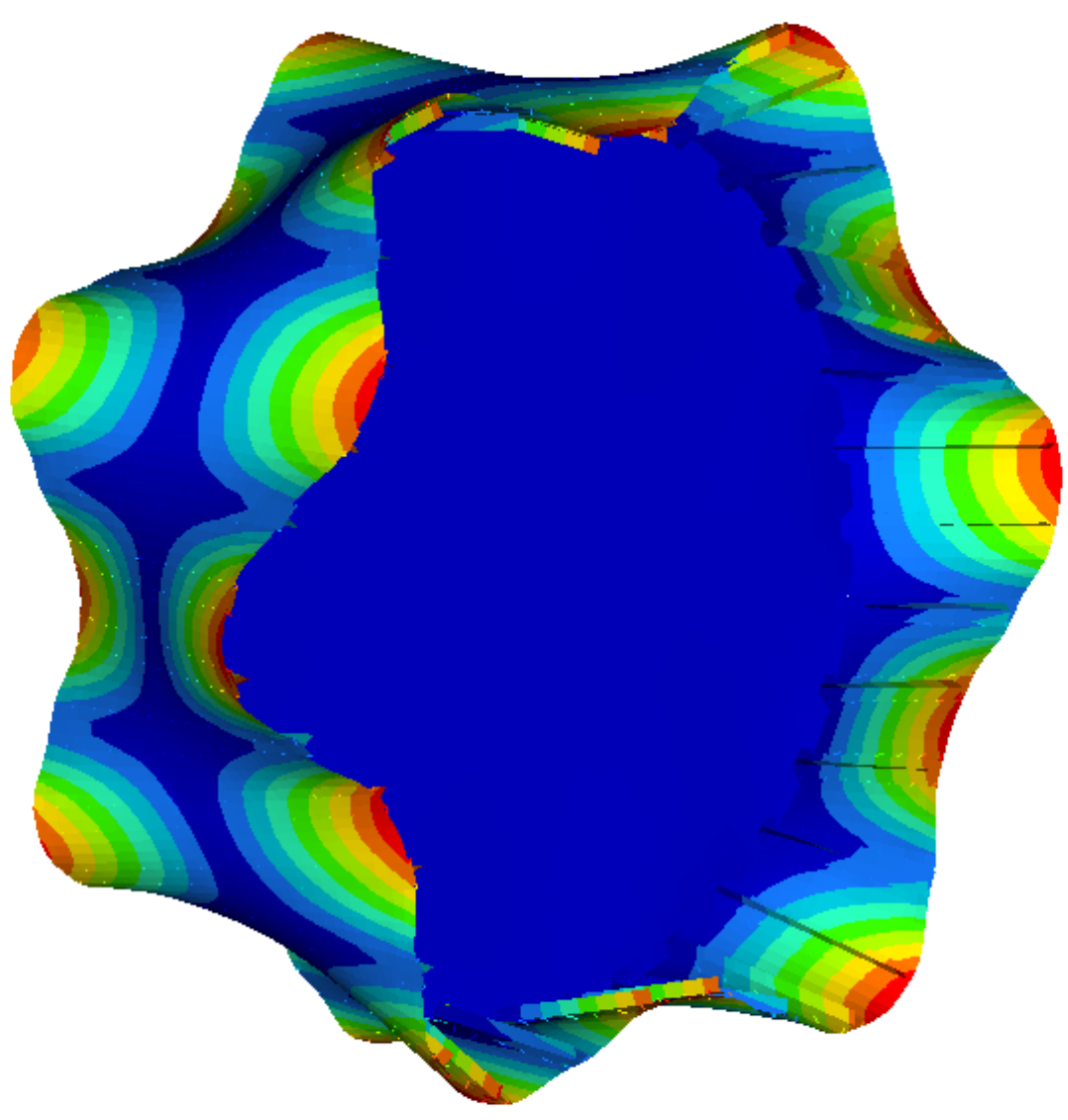

Figure 5.18: “6 Point Star Skin” FEM mode shape (CMP 10 at 172.78 Hz) (Lam \& Mechefske, 2020) 


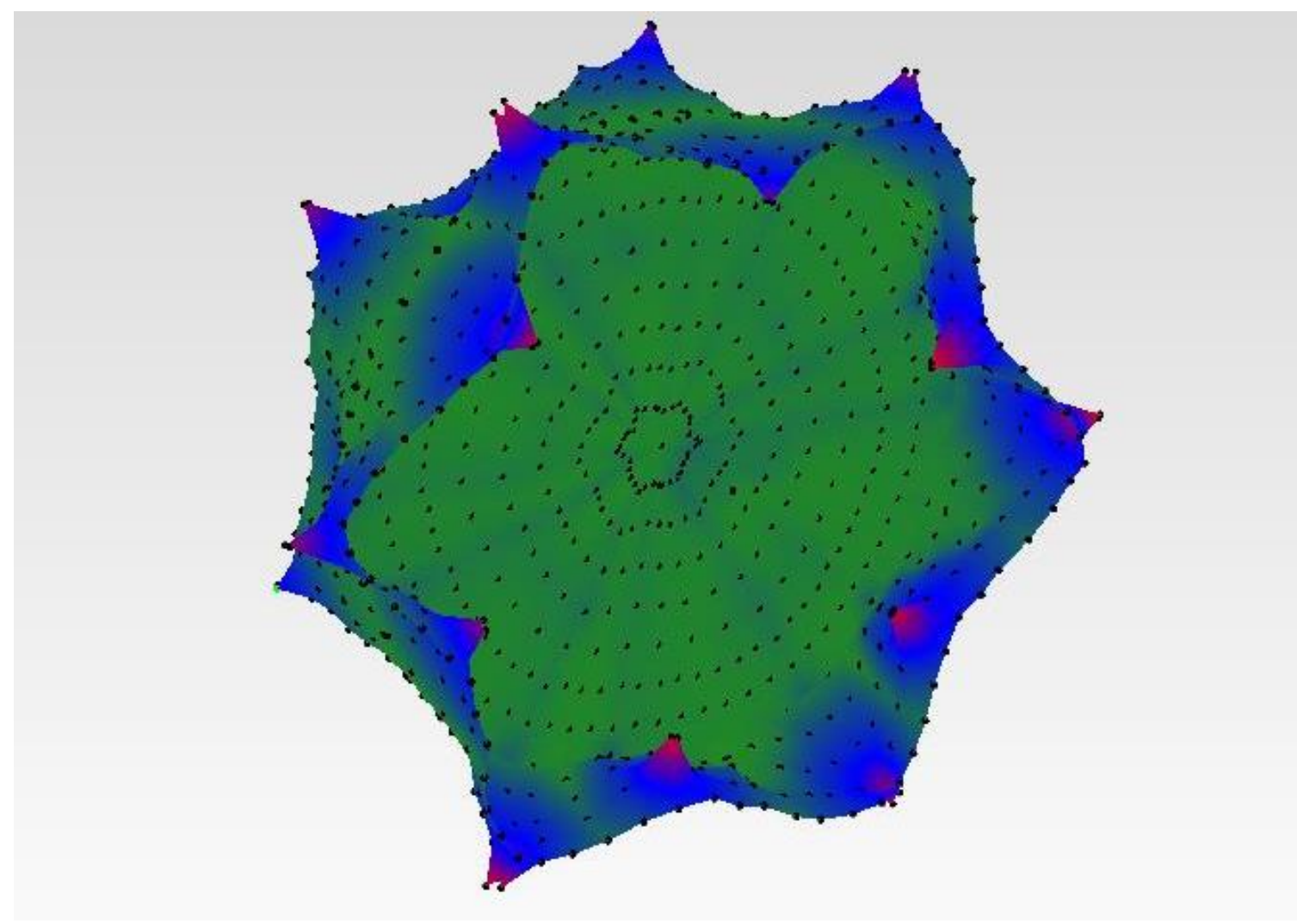

Figure 5.19: “6 Point Star Skin” Experimental mode shape (CMP 10 at 170 Hz) (Lam \& Mechefske, 2020) 


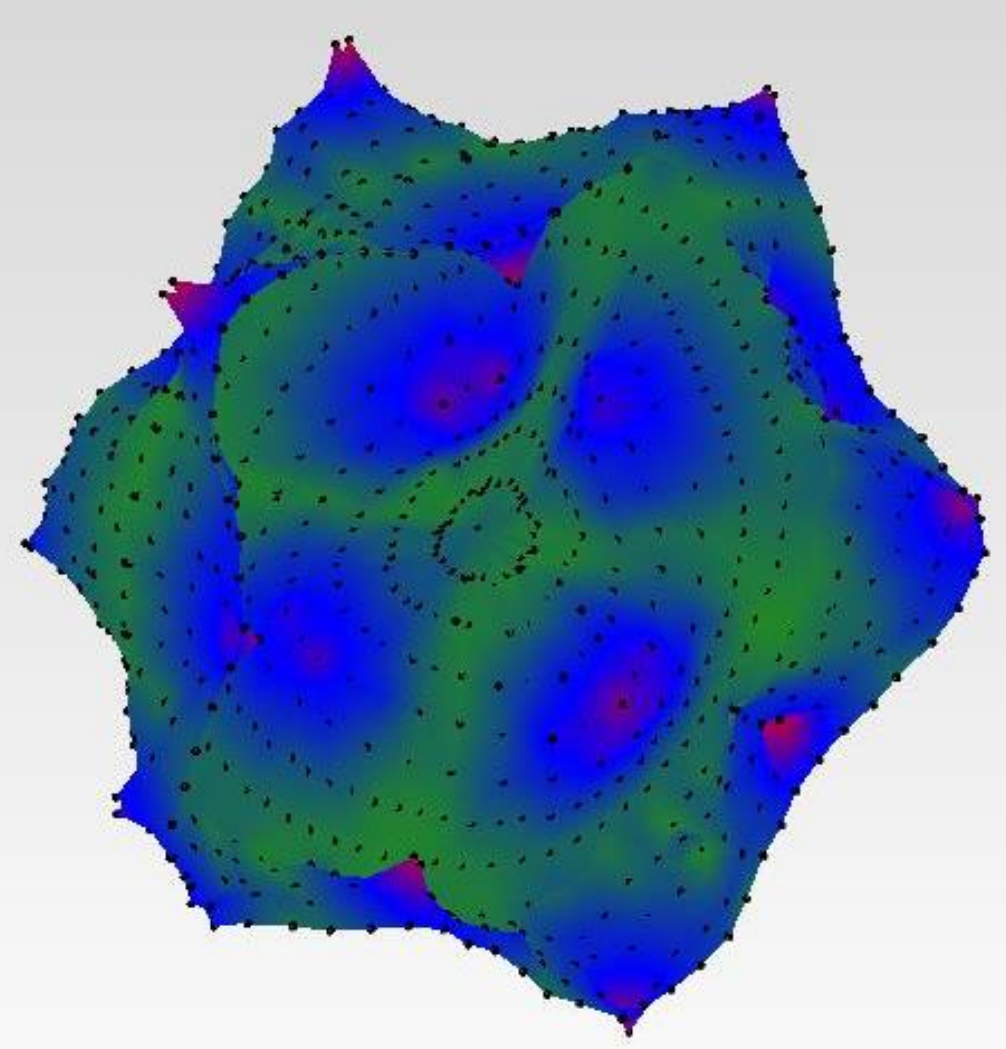

Figure 5.20: Experimental mode eight at $138 \mathrm{~Hz}$ (highlighted in yellow in Table 5.1)
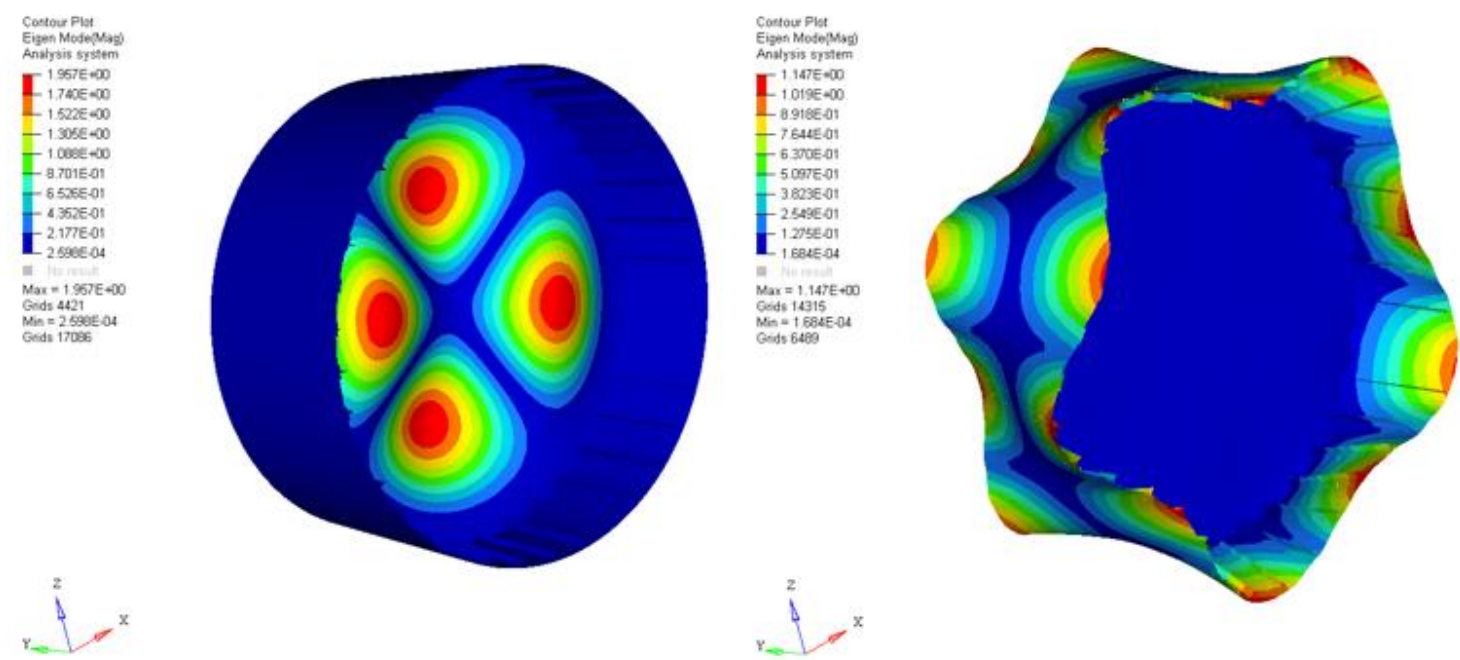

Figure 5.21: Computational Modes eight and 9 (highlighted in yellow in Table 5.1) (Lam \& Mechefske, 2020) 
Once the mode shapes have been visually correlated between the two sets of results, the quality of correlation must be quantified. The POC was used to compute the level of correlation, with good correlation being indicated by diagonal terms above 0.8 and off diagonal terms below 0.2. The results from the POC implemented on the results from sub-assembly one are shown in Figure 5.22. It is found that all diagonal terms in the POC matrix are above 0.94 and all off diagonal terms are below 0.2 with the exception of the term from the comparison of experimental mode 11 with computational mode 10 which had a value of 0.21 . It can be concluded that for the eleven modes presnted below, there is a strong level of correlation. This confirms both that the correct natural frequencies are being compared, and that the computational model is correctly predicting the overall dynamic behaviour of sub-assembly one.

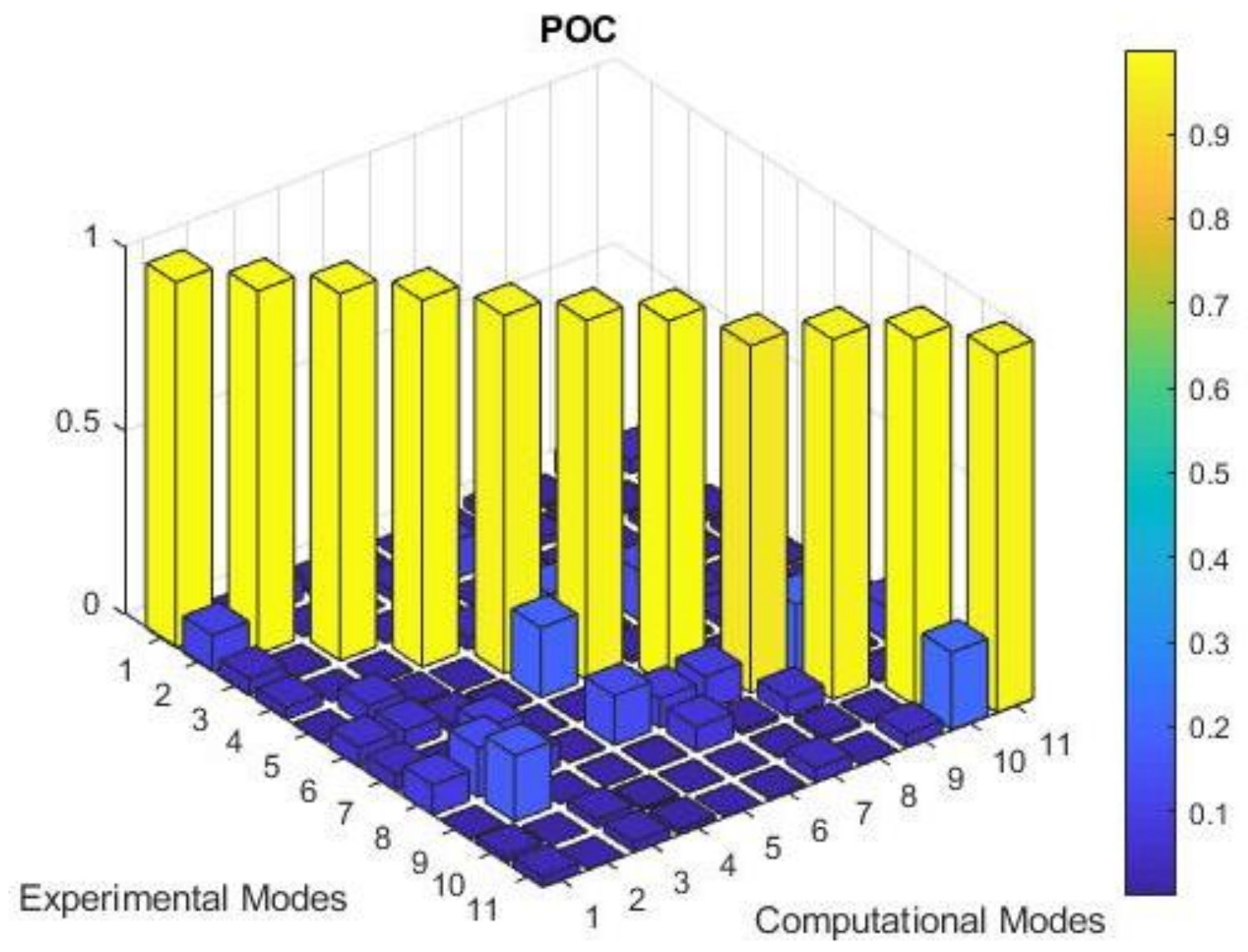

Figure 5.22: POC computation for 11 correlated modes of sub-assembly one. 


\subsection{Sub-Assembly Two Natural Frequency Correlation}

Due to the modal density of the second sub-assembly in the $200 \mathrm{~Hz}$ bandwidth, the results from both engine support frames were separated prior to analysis and correlation. The procedure to evaluate the correlation between experimental and computational results followed the same steps as used for the first sub-assembly where mode shapes were visually related, their respective natural frequencies compared, at which point the POC was used to determine the quality of mode shape correlation for the mode shapes compared. The comparison of natural frequencies for sub-assembly two is presented in Table 5.2.

Table 5.2: Comparison of sub-assembly 2 natural frequencies

\begin{tabular}{|c|c|c|c|}
\hline CMP FESF & Experimental Results (Hz) & Computational Results (Hz) & Percent Difference (\%) \\
\hline 1 & 21 & 15.17 & -27.74 \\
\hline 2 & 33.8 & 20.78 & -38.52 \\
\hline 3 & 41.6 & 30.24 & -27.32 \\
\hline 4 & 46.8 & 43.35 & -7.38 \\
\hline 5 & 66.5 & 60.83 & -8.52 \\
\hline 6 & 79.1 & 76.99 & -2.67 \\
\hline 7 & 96.5 & 94.27 & -2.29 \\
\hline 8 & 115 & 105.82 & -7.98 \\
\hline 9 & 127 & 124.22 & -2.19 \\
\hline 10 & 137 & 133.31 & -2.69 \\
\hline 11 & 151 & 138.29 & -8.42 \\
\hline 12 & 158 & 149.01 & -5.69 \\
\hline 13 & 163 & 151.89 & -6.81 \\
\hline 14 & 170 & 168.90 & -0.65 \\
\hline 15 & 188 & 175.67 & -6.56 \\
\hline CMP RESF & Experimental Results (Hz) & Computational Results (Hz) & Percent Difference (\%) \\
\hline 1 & 25.5 & 17.66 & -30.75 \\
\hline 2 & 32.5 & 20.61 & -36.58 \\
\hline 3 & 41.6 & 36.37 & -12.57 \\
\hline 4 & 75.8 & 66.43 & -12.36 \\
\hline 5 & 89 & 85.05 & -4.44 \\
\hline 6 & 136 & 119.71 & -11.98 \\
\hline 7 & 153 & 141.87 & -7.28 \\
\hline 8 & 157 & 147.11 & -6.30 \\
\hline 9 & 177 & 159.02 & -10.16 \\
\hline 10 & 197 & 176.13 & -10.59 \\
\hline
\end{tabular}


From the results in Table 5.2, there is clearly an issue with the experimental frequencies of CMPs 1 - 3 of the FESF and the first two CMPs of the RESF. When evaluating the natural frequency comparison of the first sub-assembly, there was a relatively consistent magnitude of error between computational and experimental results, however this was not the case for the second sub-assembly. There is an acceptable level of error for most CMPs, but the first three natural frequencies of the FESF in addition to the first two CMPs of the RESF show a far greater discrepancy. It is theorized that the boundary altered the natural frequencies of the first three modes. This is based on previous research where boundary conditions were shown to have a significant effect on natural frequencies (Chamberlain, 2018). To best approximate the free-free boundary condition, soft elastic members are recommended to support the test article, however to support the weight of the structure, relatively stiff bungees were required. The second subassembly was supported through the pylons of both engine support frames which only show modal activity for the first three modes of both the RESF and the FESF. The experimental natural frequencies for these modes indicate that the bungees are imposing a stiffening effect on the structure. It is therefore recommended that the support locations be moved from the engine pylons to the sections of frame surrounding both engine support frames, which are modally inactive over the bandwidth of interest. Testing the sub-assembly using this configuration will confirm or disprove this theory with the latter scenario indicating an issue with the computational model.

It should also be noted that the computational model underpredicts every natural frequency in the bandwidth. This was not the expected result, as computational models generally overpredict stiffness and as a result, natural frequency. This discrepancy is most likely due to the outward tension imposed on both engine support frames by stress in the sections of skin. This theory is further corroborated by the larger discrepancies found in the RESF natural frequencies. The RESF has a smaller radius than the FESF, and thus the skin section must be bent further to 
conform to its shape. This results in more pre-stress in the skin, and therefore more outward tension on the RESF. Recommendations to improve this involve either pre-forming the skin to the required radii, or using aluminum of lower young's modulus for the skin sections.

\subsection{Sub-assembly Two Mode Shape Correlation}

The same procedures used to correlate the mode shapes found with sub-assembly one, were implemented when comparing mode shapes found for sub-assembly two. That is, a graphical representation of each mode shape was generated using Me'Scope, and these were then visually matched to those generated computationally. The model predicted 25 mode shapes over the $200 \mathrm{~Hz}$ bandwidth, and all 25 were visually correlated with the mode shapes found experimentally. Figures 5.23 and 5.24 present an example of the visual correlation found for CMP 3 of the FESF and Figures 5.25 and 5.26 present CMP 5 of the RESF. 


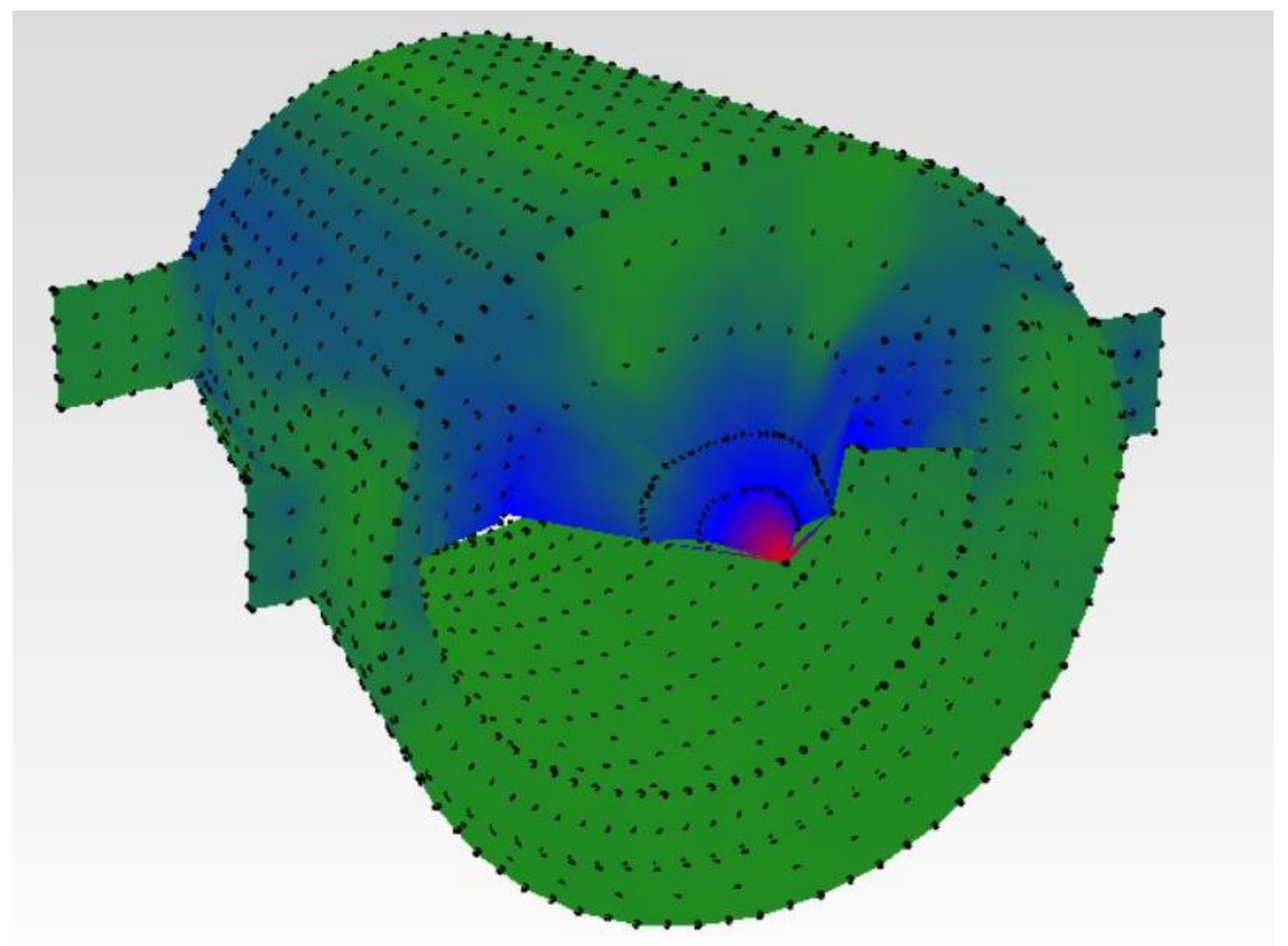

Figure 5.23: Third FESF experimental mode shape at $41.6 \mathrm{~Hz}$

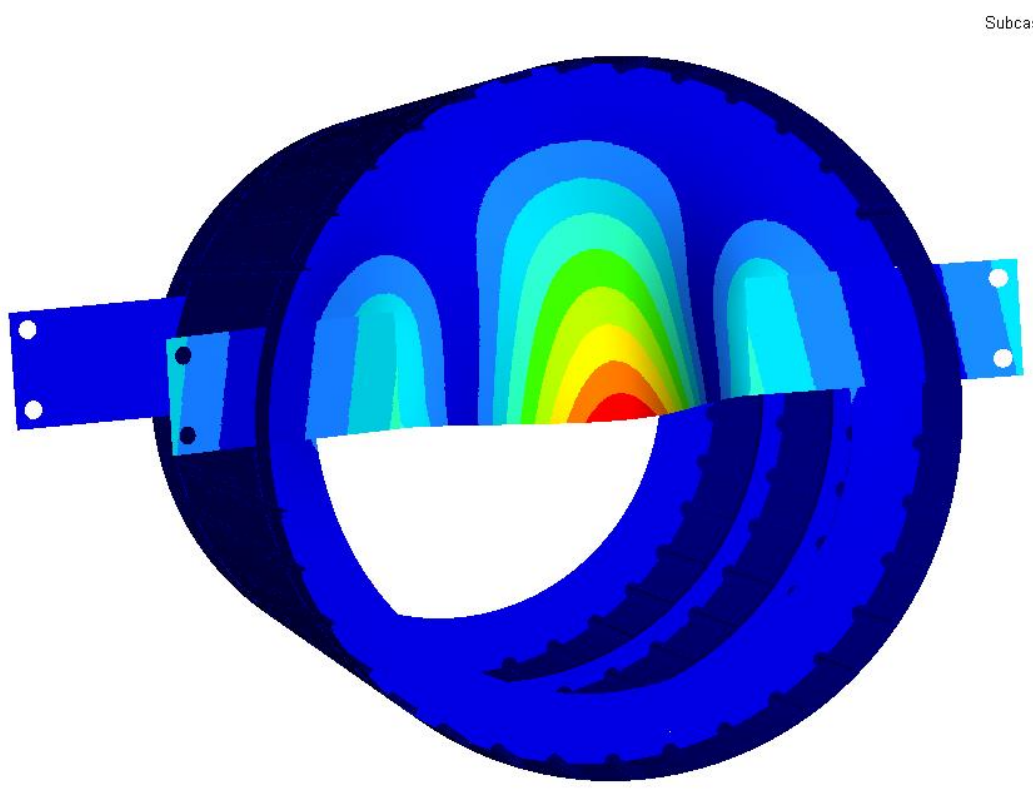

Figure 5.24: Third FESF computational mode shape at 30.24 Hz (Lam \& Mechefske, 2020). 


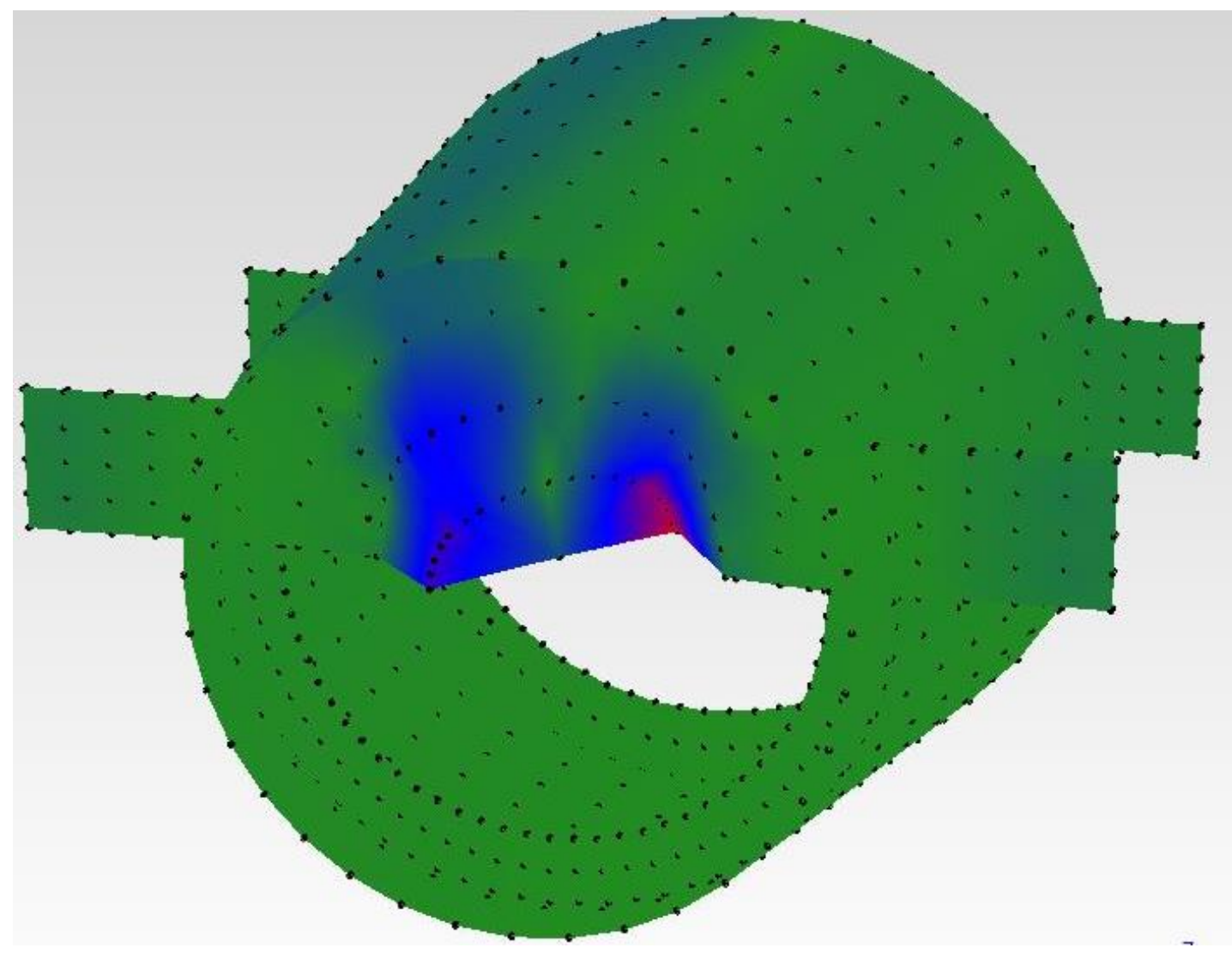

Figure 5.25: Fifth RESF experimental mode shape at $89 \mathrm{~Hz}$

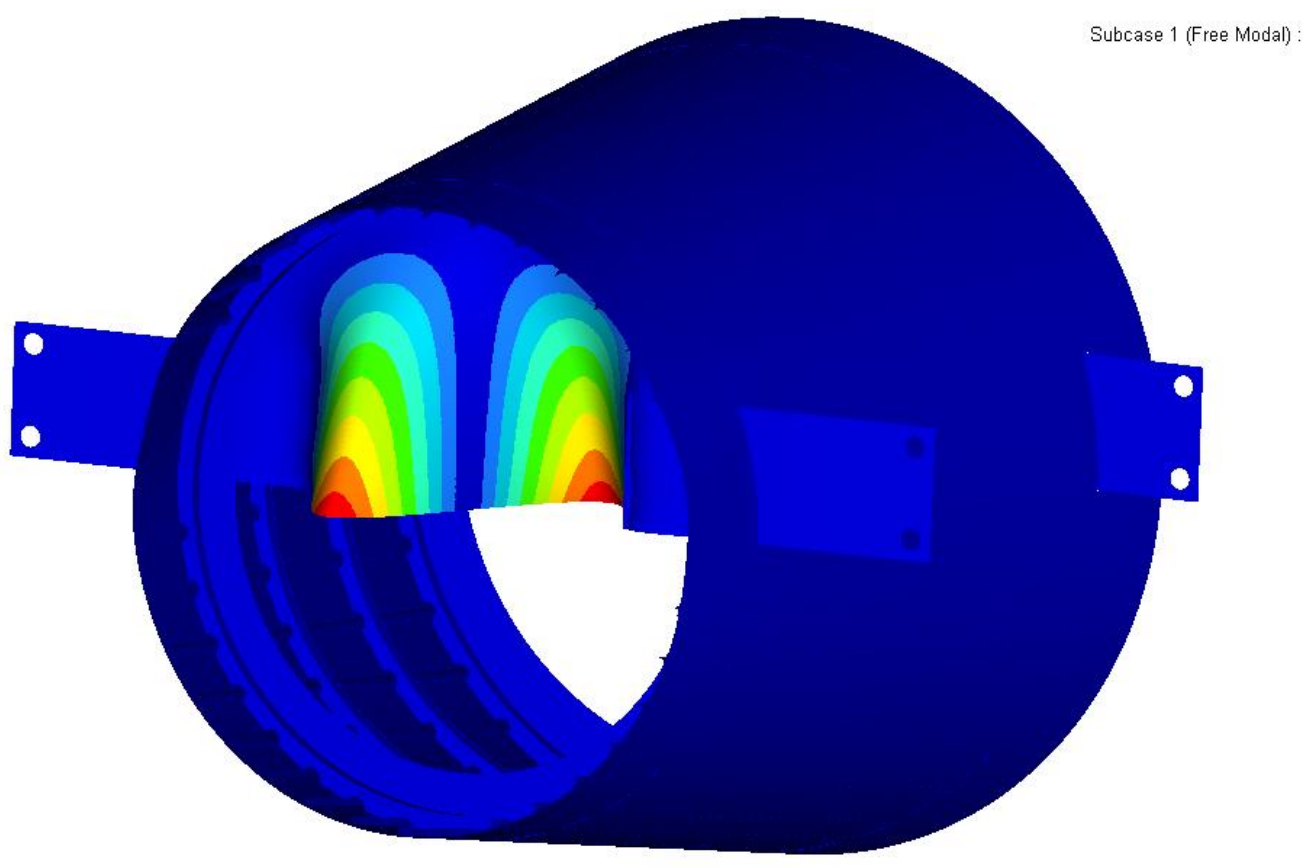

Figure 5.26: Fifth RESF computational mode shape at 85.05 Hz (Lam \& Mechefske, 2020). 101 
Once again, the POC was used to quantify the quality of correlation between the computational and experimental mode shapes. For the 15 FESF mode shapes predicted, 14 showed acceptable correlation, with all diagonal terms above 0.91 , and off diagonal terms below 0.2 except for the correlation value from mode 13 and experimental mode 11 at 0.292 . There were other high off diagonal terms including those found between modes 1 and 3, and modes 2 and 4. Due to the similarity in mode shapes, these off diagonal terms are not of concern, especially when considering the magnitude of the diagonal values related to CMPs 1-4. CMP 12 was found to be erroneous with a diagonal term of 0.675 and an off diagonal term between computational mode 11 and experimental mode 12 of nearly the same magnitude. There was a relatively low density of accelerometers in the area of resonance, and the lack of resonant motion elsewhere in the structure meant that slight discrepancies in mode shape data were exaggerated. The POC matrix for the FESF is shown in Figure 5.27, while Figures 5.28 and 5.29 illustrate the issues regarding the erroneous correlation of CMP 12.
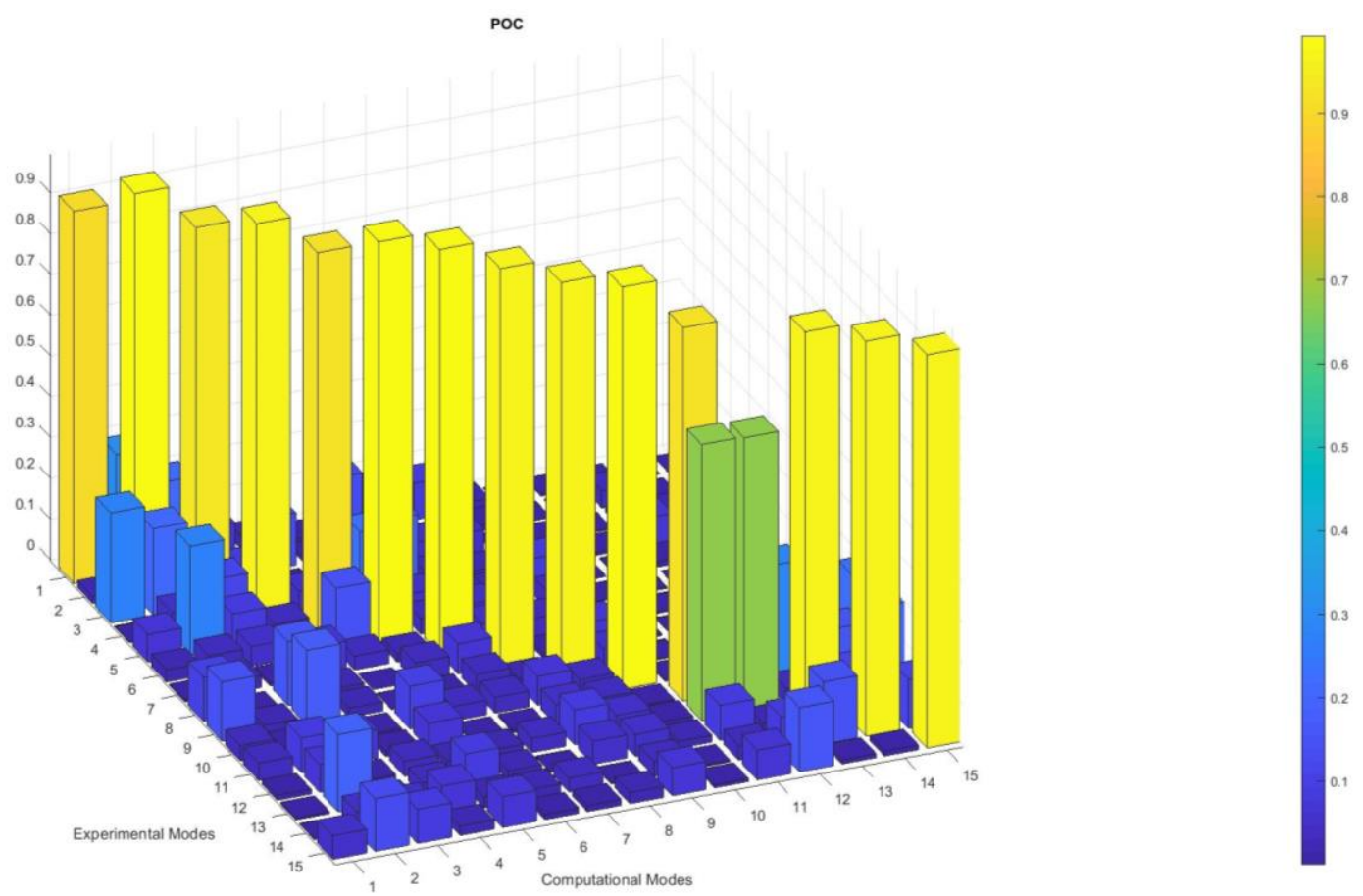

Figure 5.27: POC computation for 15 correlated modes from the FESF of sub-assembly 2. 

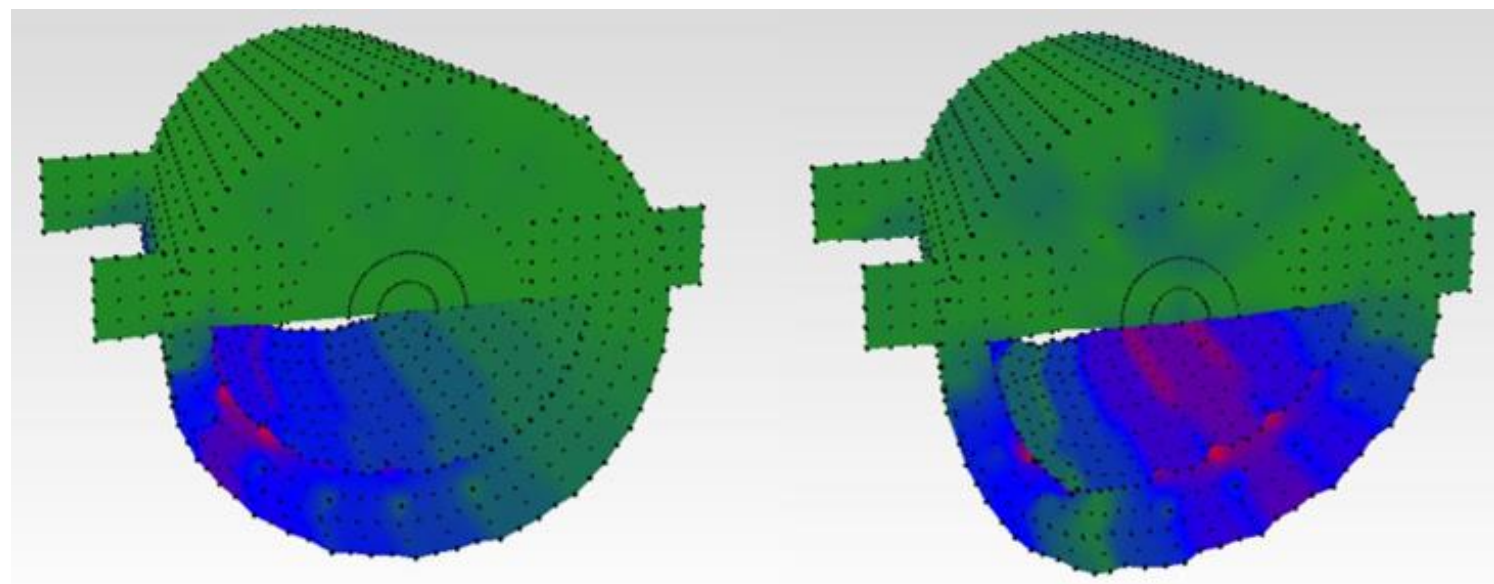

Figure 5.28: Similarity between FESF mode 11 (Left) and FESF mode 12 (right) found experimentally.
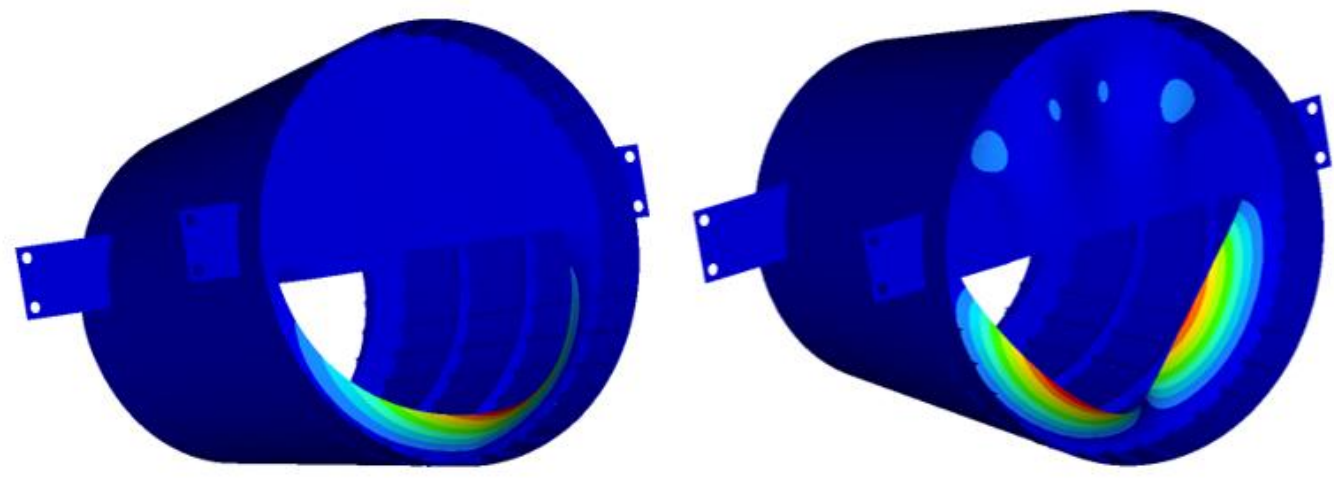

Figure 5.29: Predicted FESF modes 11 (Left) and 12 (Right) (Lam \& Mechefske, 2020).

The computational model predicted 10 mode shapes for the RESF, and 10 were found experimentally. All of these showed acceptable levels of correlation with all diagonal terms above 0.82 and most off diagonal terms below 0.3. Once again, the first three modes showed significant off diagonal terms, but due to the similarity in mode shapes, these were not of significant concern. In general, the correlation of RESF modes was not as strong as the correlation found for the FESF mode shapes. It does however show strong enough correlation to confirm that the correct natural frequencies are being compared. Figure 5.30 shows the POC matrix produced from the comparison of RESF mode shapes. 

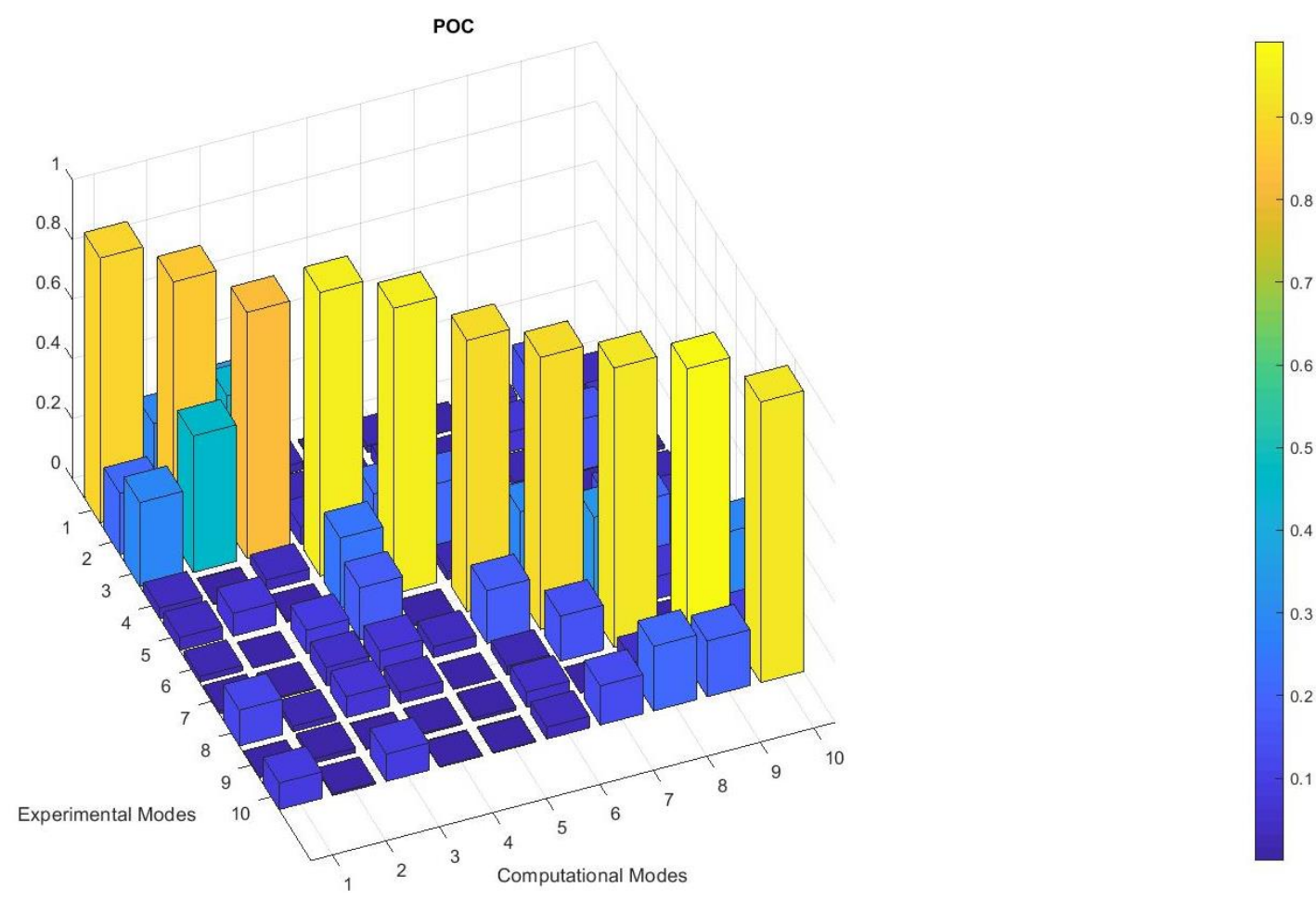

Figure 5.30: POC computation for 10 correlated modes from the RESF of sub-assembly 2.

\subsection{Chapter Summary}

This chapter provided an in-depth overview of the results produced by various validation trials, as well as those used for comparison with the computational model. A rigorous set of validation tests was implemented, including those recommended by the literature, and further experimentation to determine the optimal experimental setup for the tests performed. These tests confirmed the linearity of the test articles and repeatability of the experimental setup and procedure. Additionally, the validity checks helped to determine the best response measurement layout and confirmed that other experimental setup parameters would provide accurate results. Once the test setup and procedure were validated, modal parameters including the natural frequencies and mode shapes could be extracted with a high level of confidence. These parameters were compared with those produced by the computational model formed by (Lam \& Mechefske, 2020). In the case of the first sub-assembly, it was found that modifications were required to accurately model the dynamic behavior of the skin. Once this change was made to the 
computational model, a strong level of correlation between the natural frequencies and mode shapes was found. The second sub-assembly provided an acceptable level of correlation for natural frequencies and mode shapes, however slightly larger discrepancies were noted. It was determined that some incongruity was likely due to the computational model not accounting for the pre-stress in the skin panels, however a significant amount was likely due to the boundary conditions. A future amendment to the experimental setup was proposed to remedy this and testing with the altered boundary conditions will likely improve correlation. With this phase of the project complete, recommendations for future testing improvements can be made in chapter 6 . In addition, the next phases of the project including the expansion from sub-assemblies to a complete half scale tail section will be discussed. 


\section{Chapter 6}

\section{Conclusions and Future Recommendations}

\subsection{Summary and Conclusions}

Noise within the cabin of aircraft negatively effects the comfort and overall flying experience for passengers during flight. For business aircraft with jet engines mounted directly to the fuselage, structure borne noise contributes the majority of that experienced in the passenger cabin. Therefore, to mitigate the interior sound pressure level, it is first necessary to determine the dynamic characteristics of these structural transmission paths at the rear of the aircraft. To accomplish this, an extensive testing methodology was formed involving the experimental testing and computational modelling of individual components before expanding to sub-assemblies, then to the creation of a complete tail section model. The experimental testing presented in this thesis was directed at obtaining the modal parameters from fuselage sub-assemblies, thus forming an intermediate step between previous work performed on individual components and future tests on a full tail section assembly

Prior to experimentation, a detailed literature review was performed to obtain the necessary information pertaining to EMA of aerospace structures. This uncovered a multitude of potential testing options, from which the final methodology was chosen. Additionally, relevant background theory was reviewed, and provided the necessary education to perform a successful modal experiment. Finally, this review uncovered information regarding data interpretation, which would prove invaluable when analyzing and correlating the experimental data. Additional pre-test work involved the construction of two fuselage sub-assemblies, the designs of which were formed as sections of a full tail section model designed by (Chamberlain, 2018). The design of the first assembly included a section of skin and a central bulkhead, such that a complete experimental methodology could be formed. The second sub-assembly was created in accordance 
with recommendations made by Chamberlain that a "Secondary larger subassembly consisting of at least two separate components connected by stringers and skin such as the RESF and/or frame rings." be tested (Chamberlain, 2018). For both sub-assemblies, a review of the individual components was presented, and recommendations for future manufacturing considerations were provided.

The structures tested in this thesis represented a significant increase in size and complexity over the components tested during the first phase of the project. As a result, it was deemed necessary that a detailed experimental methodology be formed around the testing of complex fuselage structures to obtain modal parameters from both sub-assemblies and in preparation for the next stage of the project. The transition from impact hammer to modal shaker introduced new testing parameters, some of which were selected using findings from the aforementioned literature review. Others including the choice of stinger length and the decision to use dummy masses were specific to this thesis and needed to be validated during preliminary testing. Other validity checks including repeatability and linearity measurements ensured that both the test setup and structures behaved as expected, and that the experimental results would be reliable.

The results from the first sub-assembly indicated an issue with the computational model when considering the skin modes of the system. It was found that the computational model produced by (Lam \& Mechefske, 2020). Under predicted the natural frequencies of these modes by an average of $11.74 \%$. Multiple model updating techniques were trialed, and the discrepancies between natural frequencies of the skin modes were reduced to an average of $1.17 \%$. The bulkhead modes also showed an acceptable level of discrepancy in both preliminary and updated versions of the computational model lending further confidence to the results produced. When comparing both sets of mode shapes, a strong level of correlation was found for each CMP, thus confirming that the correct natural frequencies were compared. It is therefore evident, that the 
experimental results were essential to improving the computational model of sub-assembly one, and after modifications were made, a strong level of correlation was found.

With regards to the second sub-assembly, natural frequencies and mode shapes of both engine support frames were compared. Of the 25 CMPs 20 showed acceptable correlation in natural frequency, and five were found to be erroneous. It was theorized that the boundary conditions used to support the structure were imposing a stiffening effect on certain modes thus raising their natural frequency. It was proposed that the boundary conditions be modified to remedy this, and the testing involved to evaluate this change is included in the future work. Twenty four of the twenty-five predicted mode shapes were found in the experimental data and showed acceptable mathematical correlation. While not as strong as the correlation found with sub-assembly 1 , the results from the POC still indicated that the correct natural frequencies were being compared. Finally, trends in the discrepancies between natural frequencies were discussed, and potential model improvements were proposed to further reduce discrepancies between experimental and computational results.

In conclusion, two primary objectives for this project were established, including the formation of a comprehensive testing methodology and the production of detailed and reliable experimental results were outlined. The experimental methodology described was successfully implemented to extract the modal parameters including natural frequencies and mode shapes from aircraft fuselage sub-assemblies. The extensive validity checks performed as part of this process lent a high level of confidence to the results obtained. These results were then used as a point of comparison for computational models, and where applicable, modifications were made to reduce discrepancy. At this stage, the methodology formed can be implemented on larger fuselage structures as described in the following section. It should be noted that with every modal test comes a unique set of challenges to overcome, however the procedure outlined along with some minor modifications if necessary, will provide acceptable experimental results. 


\subsection{Future Recommendations}

The next stage of this project involves extending the test structure to a complete half scale model of the tail section as shown in Figure 3.26. This would complete the direct structure borne vibration paths between the jet engines and passenger cabin and provide a more comprehensive picture of the dynamic behavior of this section of fuselage. Additionally, this structure will facilitate the simulation of other noise generating components often placed in the tail section, including hydraulic pumps and the auxiliary power unit. In addition to providing experimental results for comparison with computational models, this structure would also facilitate the evaluation of novel sound and vibration mitigation techniques, including those proposed by (Warwick, Mechefske, \& Kim, 2019).

Work in the more immediate term involves the modification to the boundary conditions of sub-assembly 2 which involves moving the supporting bungee cords from the pylons to the section of structure where the skin connects to the engine support frames, followed by a test of this structure to evaluate the effects. It is expected that the erroneous natural frequencies will shift as a result and improve correlation for the first three CMPs of the FESF and the first two CMPs of the RESF. If this is not the case however, then it must be assumed as a result of the extensive experimental validity checks, that the computational model to some extent is erroneous, but as a result of the strong correlation found with the other CMPs of sub-assembly 2 and the correlation found with sub-assembly 1 , this is not likely to be the case. Future work with respects to the computational side of this project are described in detail by (Lam \& Mechefske, 2020). 


\section{Appendix A \\ Sub-assembly One Experimental Mode shapes}

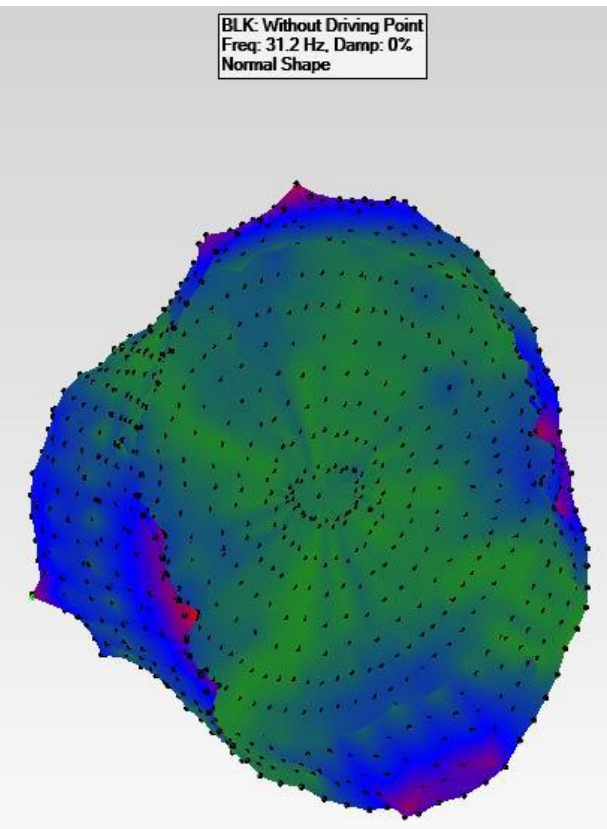

Figure A. 1: Mode 1 - Oval skin @ 31.2Hz

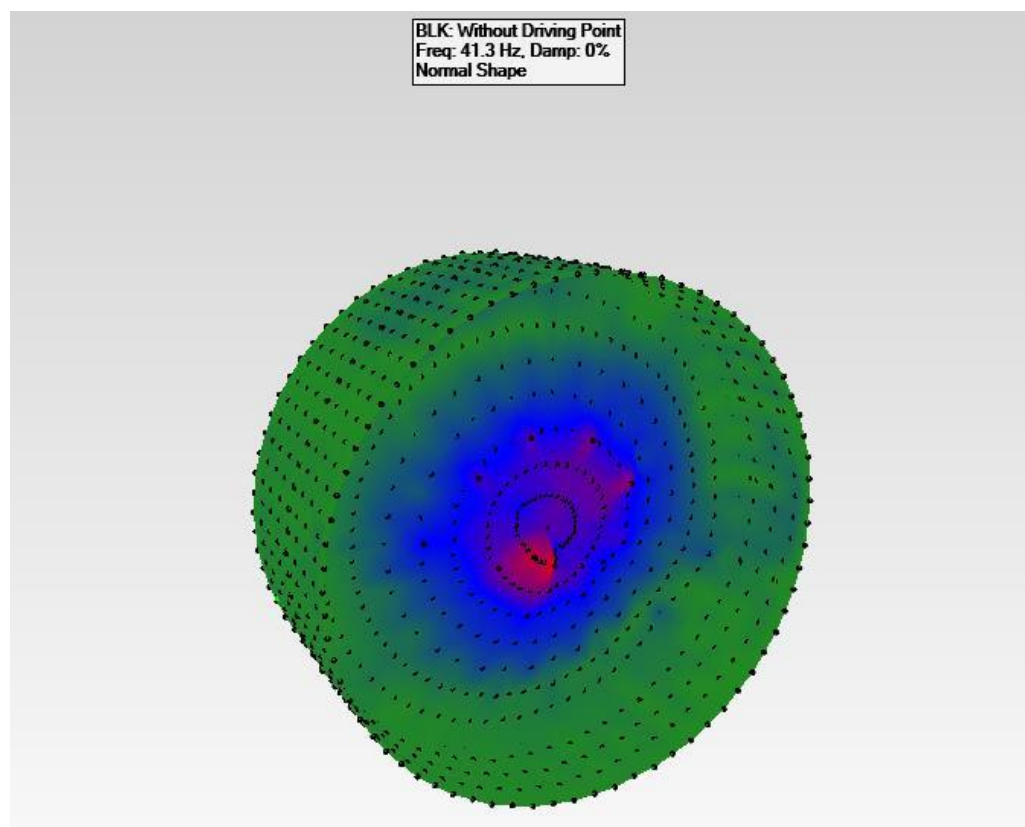

Figure A. 2: Mode 2-Bulkhead single drum @ $41.3 \mathrm{~Hz}$ 


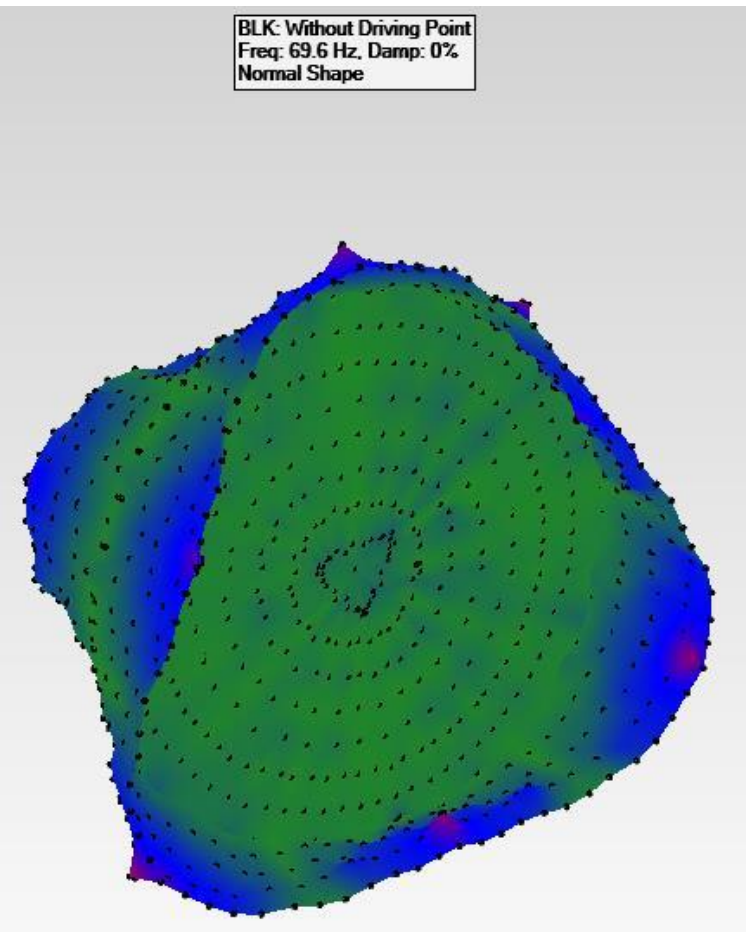

Figure A. 3: Mode 3 - Triangle skin 1 @ $69.6 \mathrm{~Hz}$

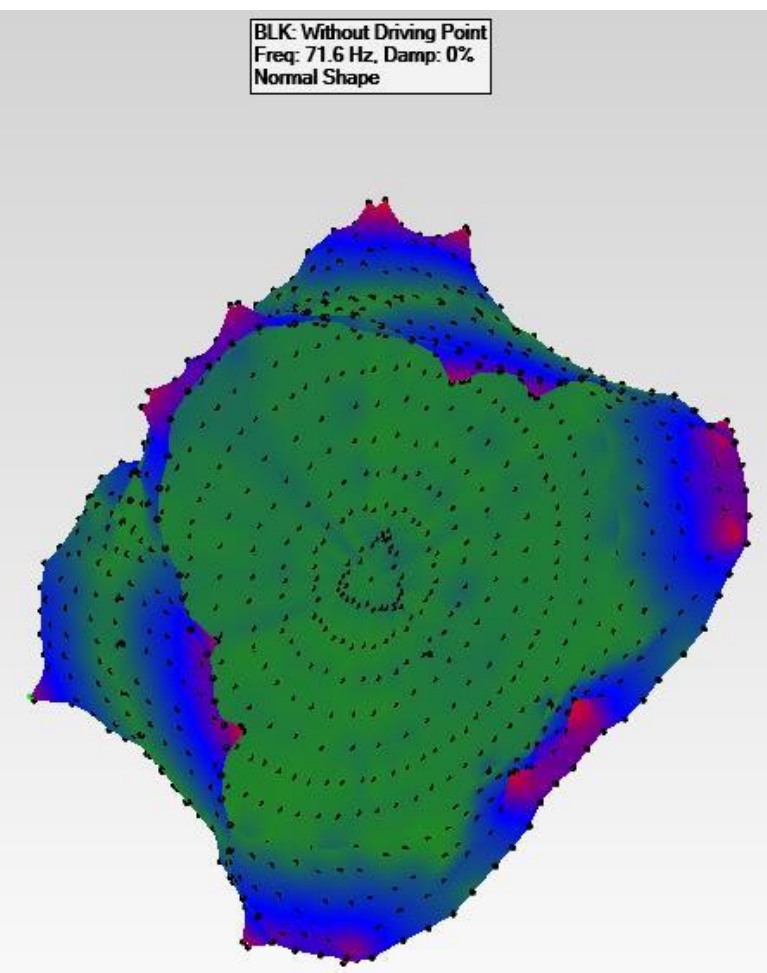

Figure A. 4: Mode 4 - Triangle skin 2 @ $71.6 \mathrm{~Hz}$ 


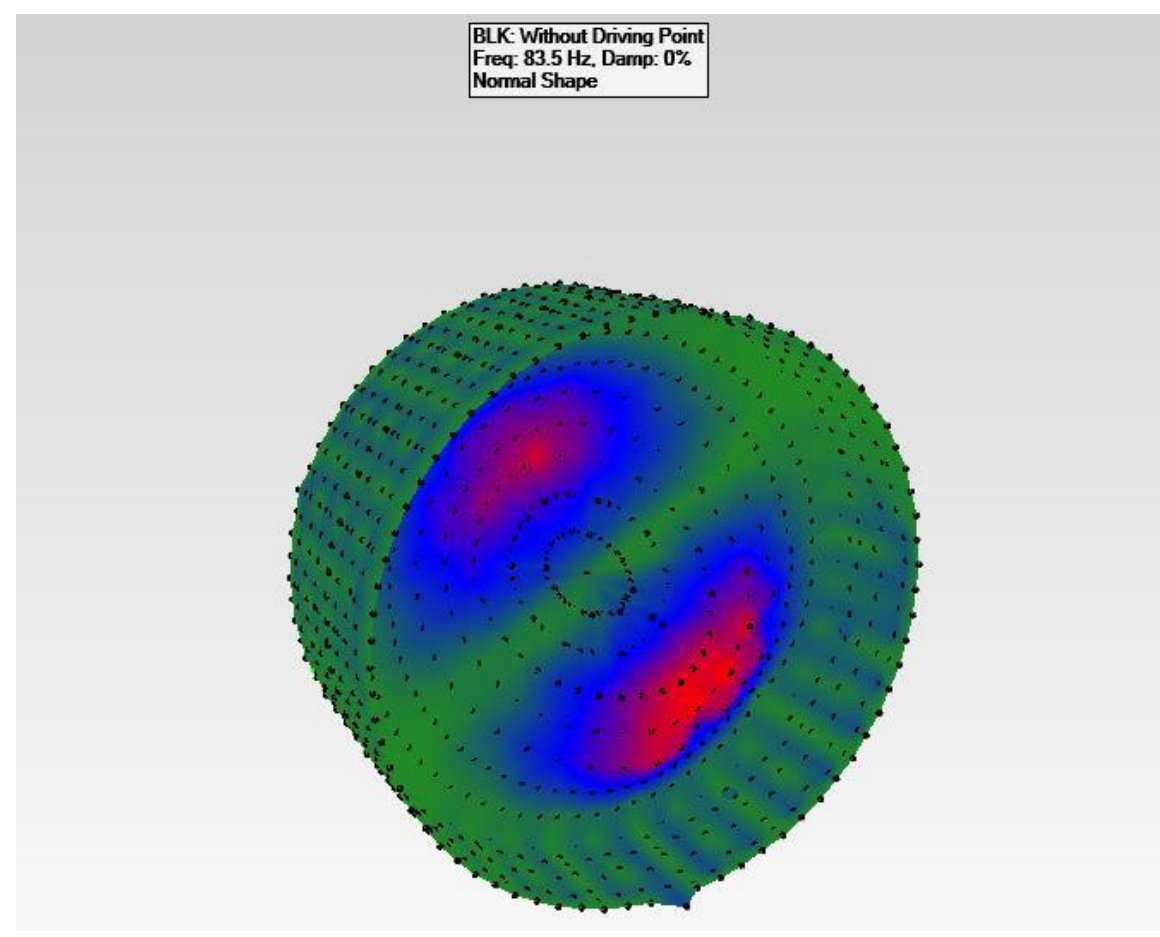

Figure A. 5 Mode 5 - Bulkhead halves $1 @ 83.5 \mathrm{~Hz}$

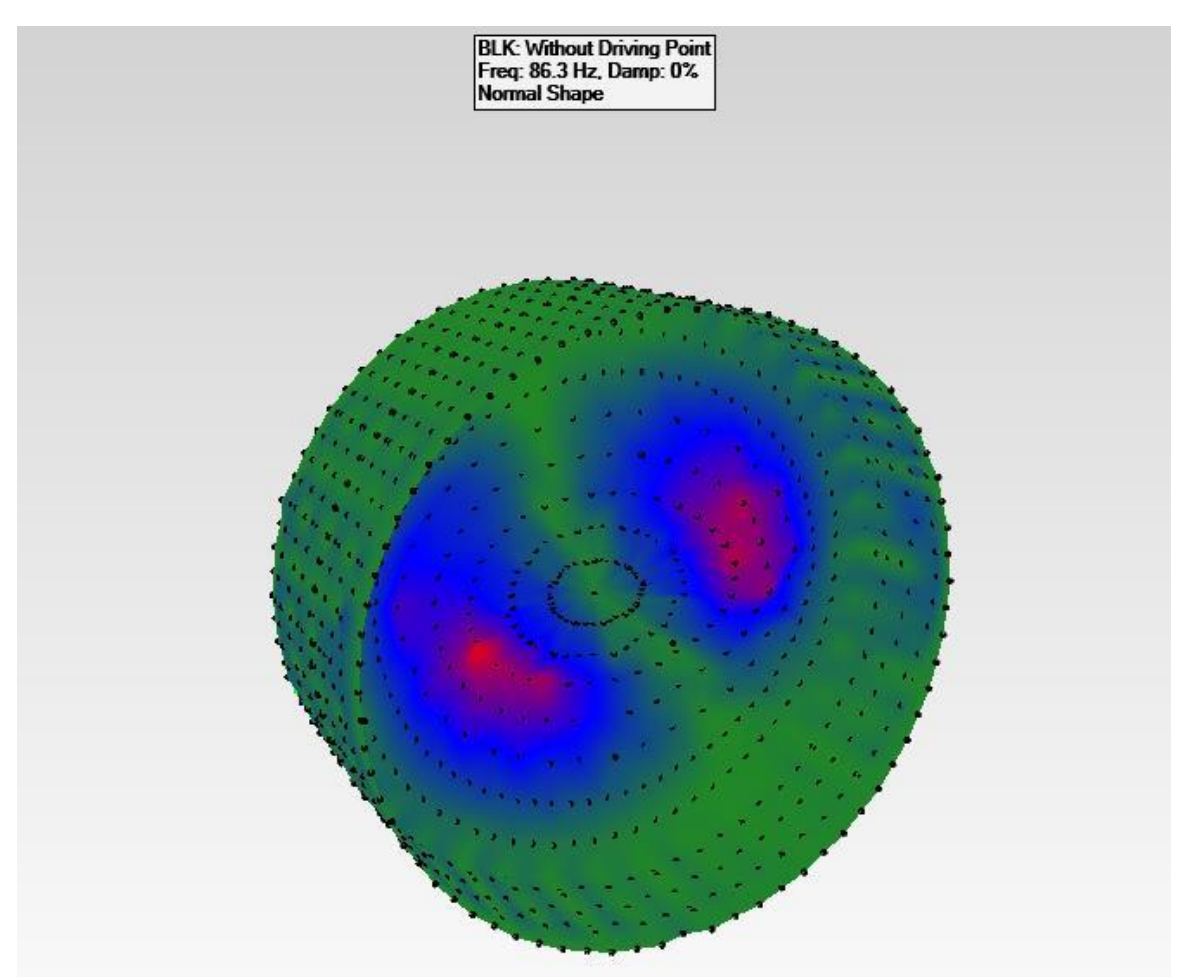

Figure A. 6: Mode 6 - Bulkhead halves 2 @ $86.3 \mathrm{~Hz}$ 


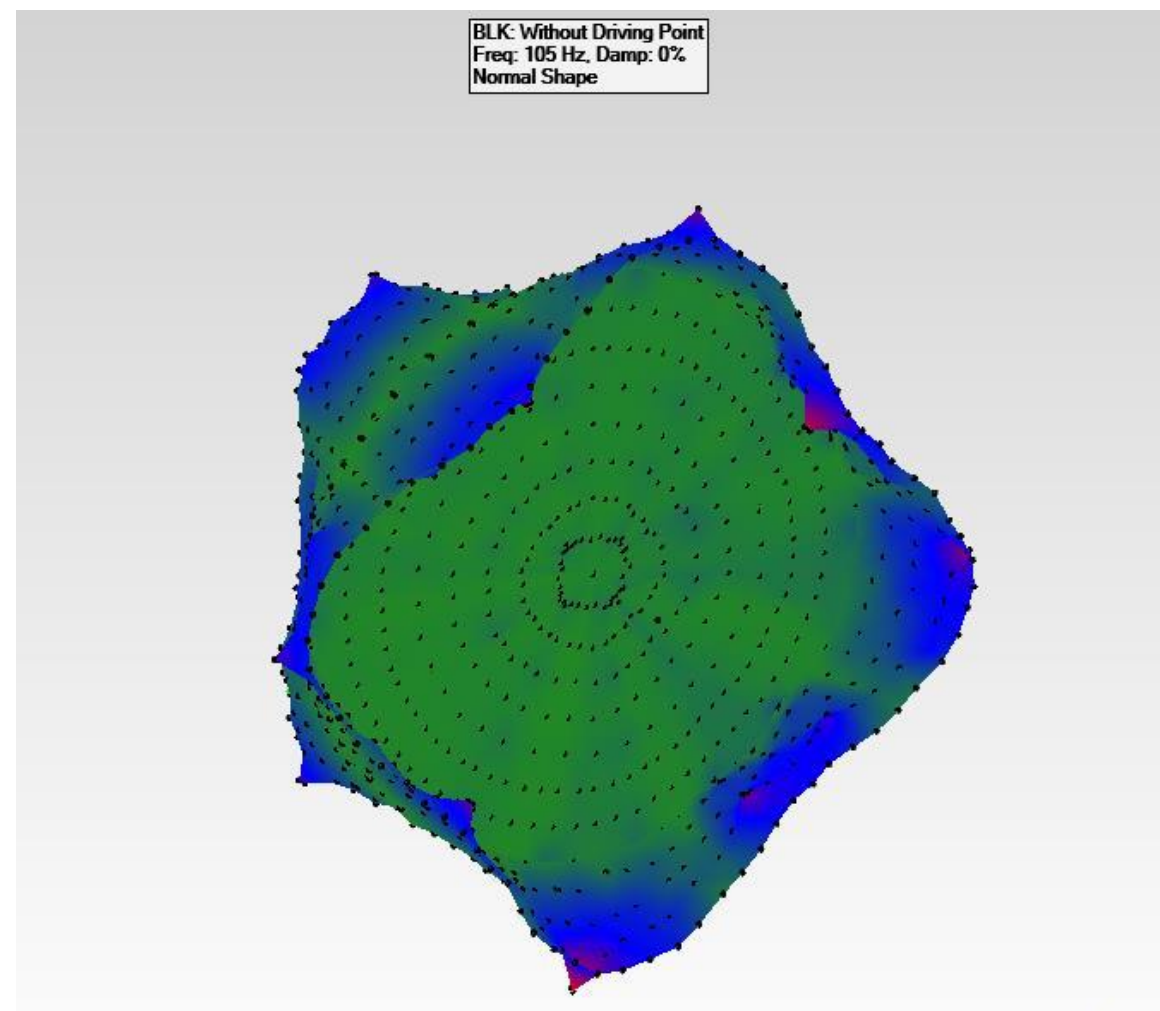

Figure A. 7: Mode 7 - Square skin @ $105 \mathrm{~Hz}$

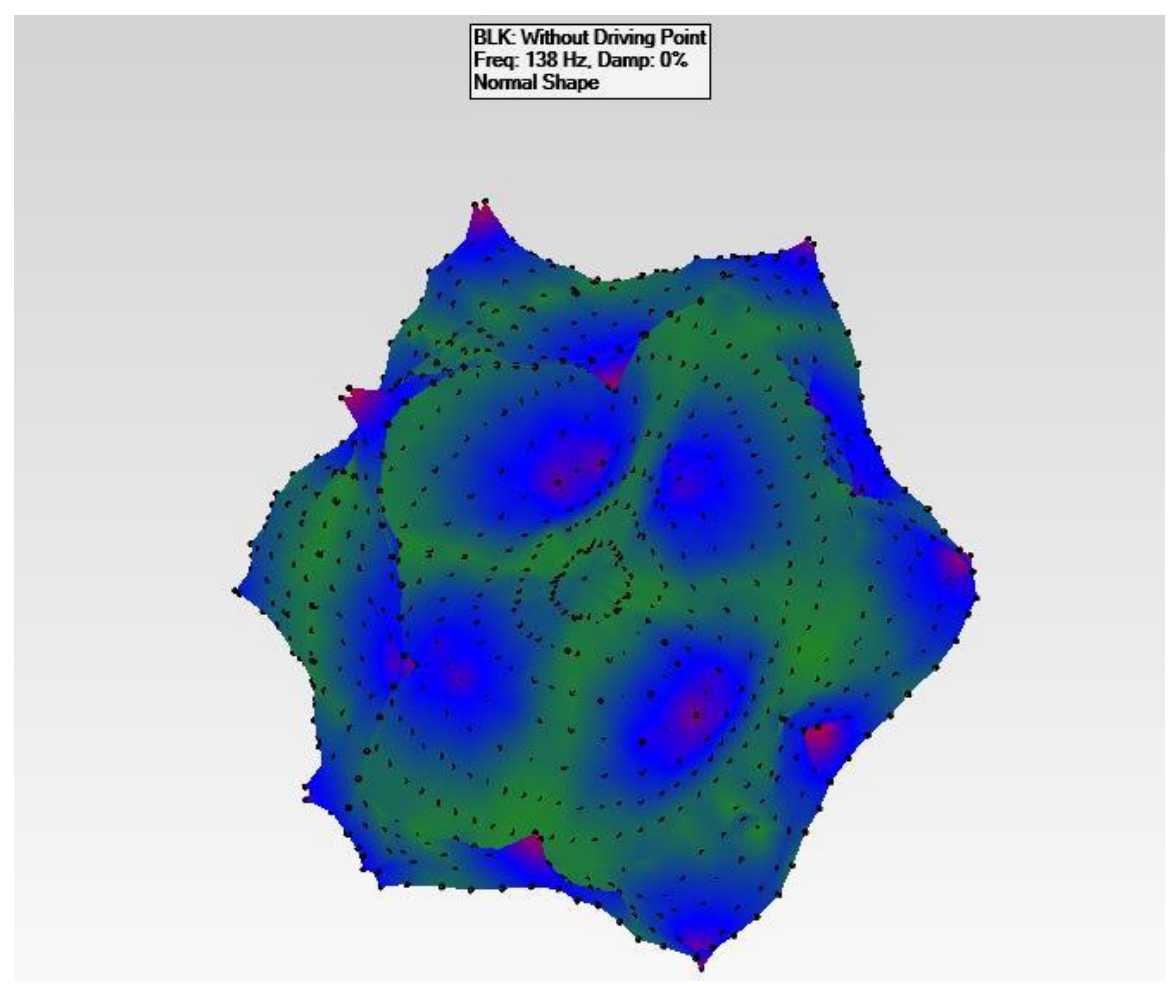

Figure A. 8: Mode 7 - Five point skin / Bulkhead quarters 1 @ 138 Hz 


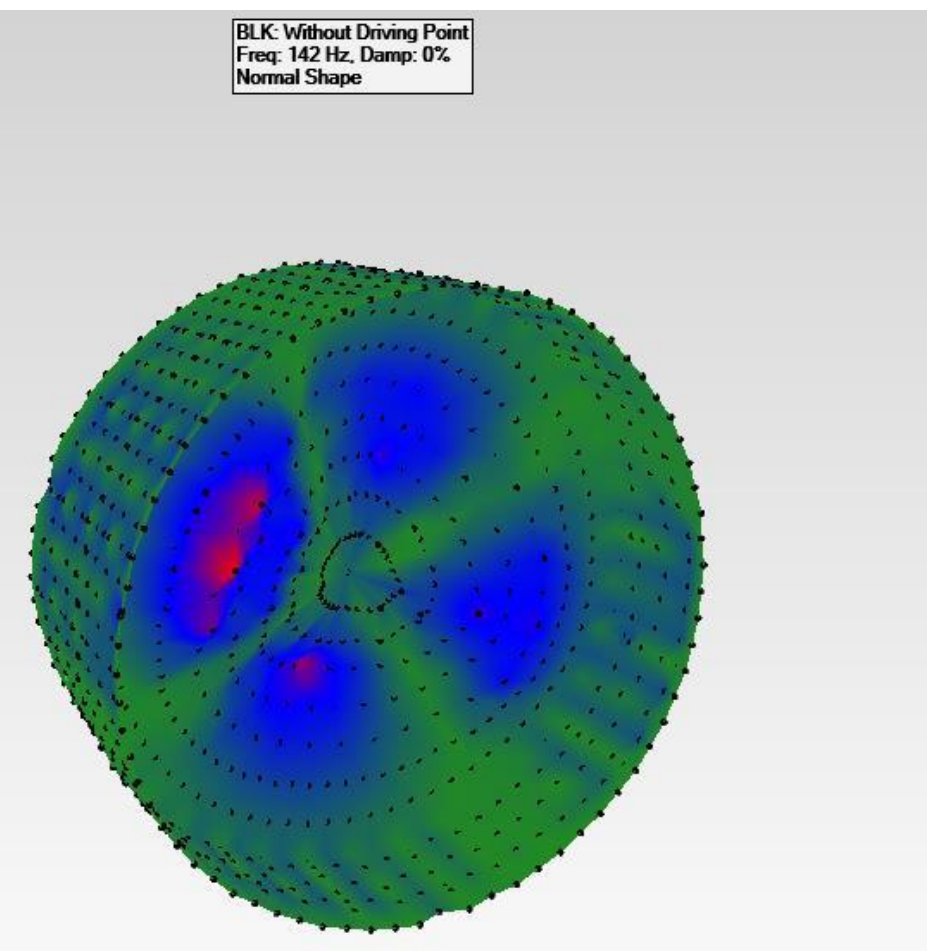

Figure A. 9: Bulkhead quarters 2 @ $142 \mathrm{~Hz}$

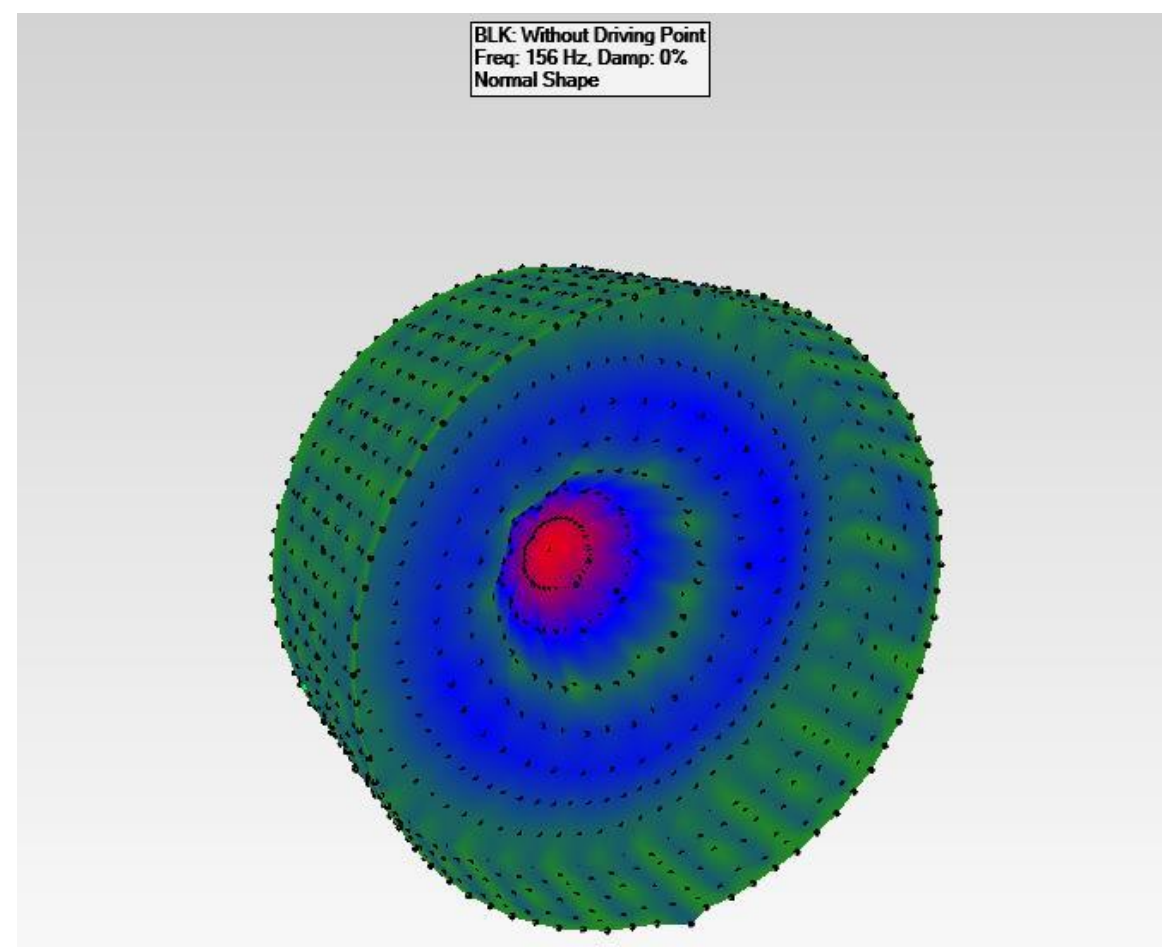

Figure A. 10: Bulkhead double drum @ $156 \mathrm{~Hz}$ 


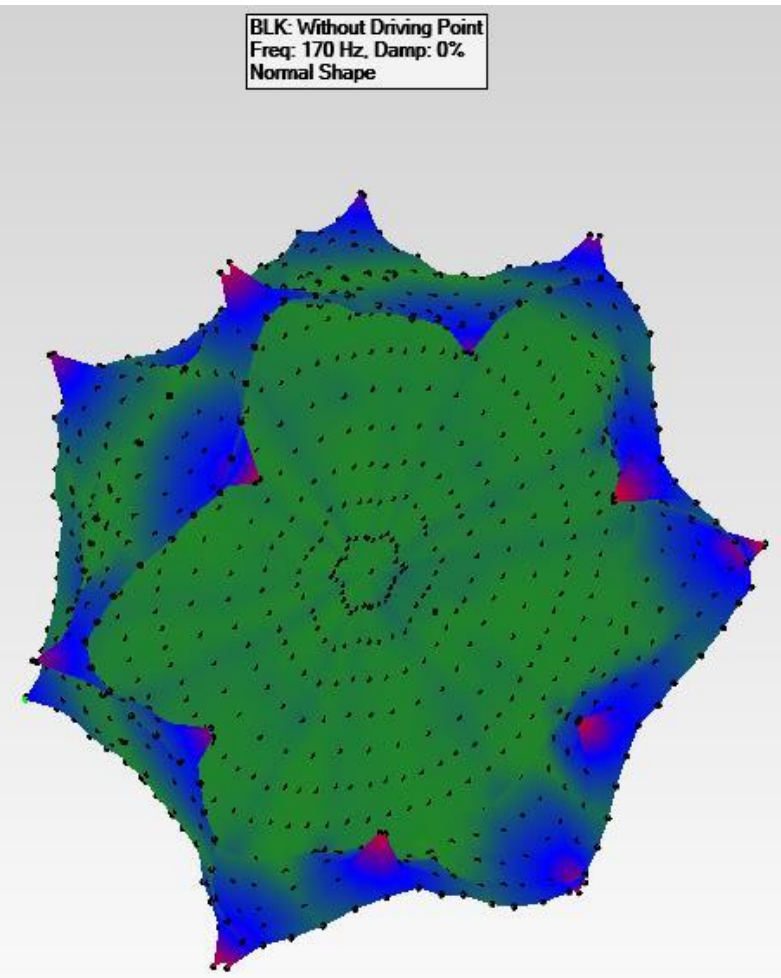

Figure A. 11: 6 point skin 1 @ $170 \mathrm{~Hz}$

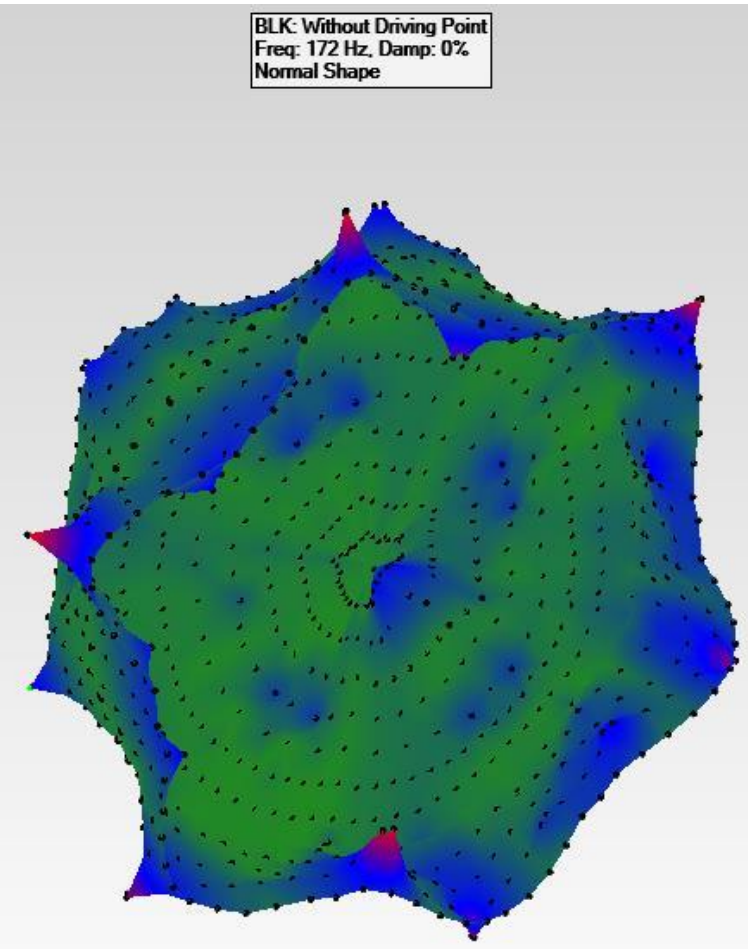

Figure A. 12: 6 point skin 2 @ $172 \mathrm{~Hz}$ 


\section{Appendix B \\ Sub-assembly Two Experimental Mode Shapes}

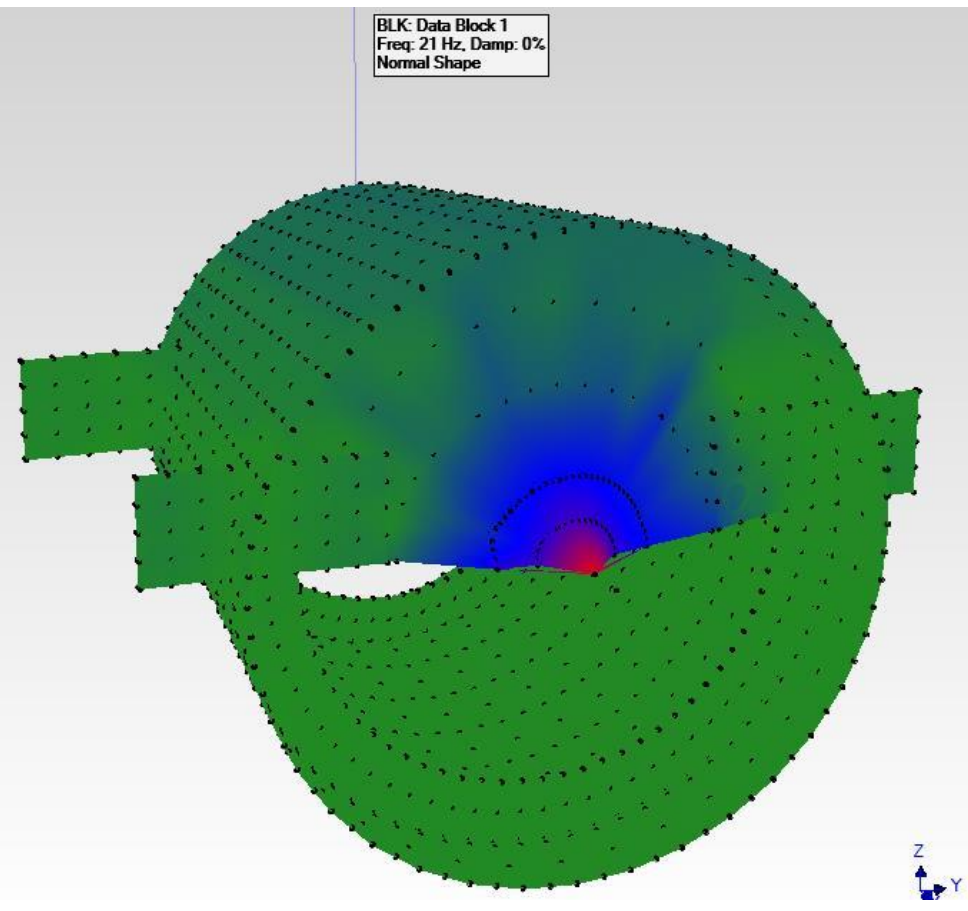

Figure B. 1: FESF Mode 1 @ $21.0 \mathrm{~Hz}$

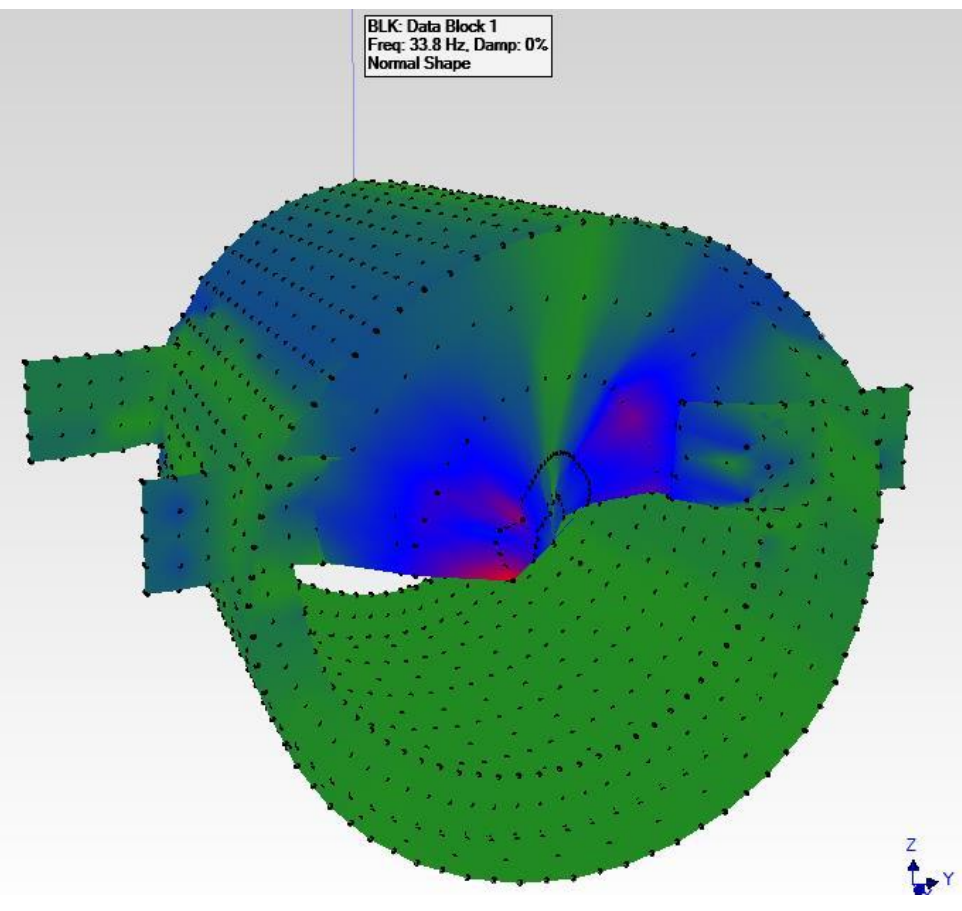

Figure B. 2: FESF Mode 2 @ $33.8 \mathrm{~Hz}$ 


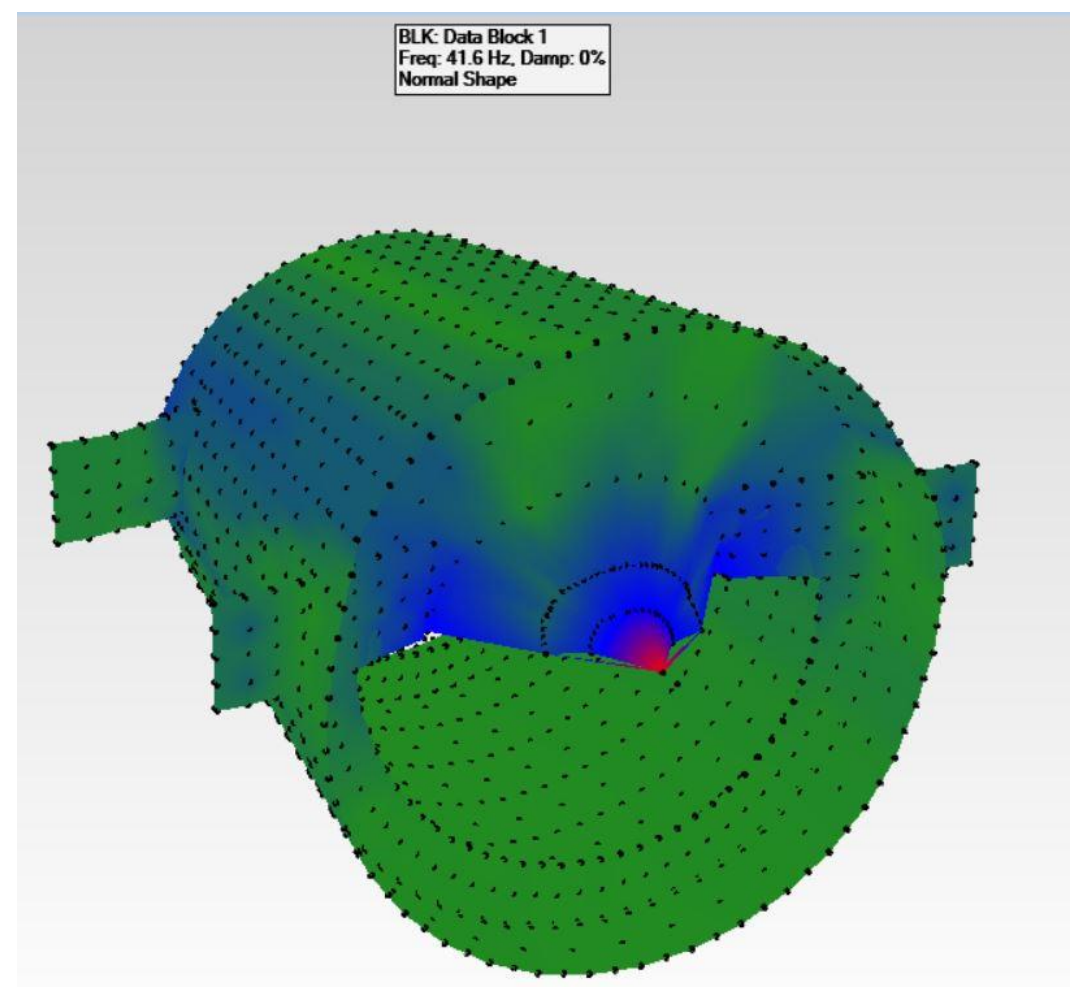

Figure B. 3: FESF Mode 3 @ 41.6 Hz

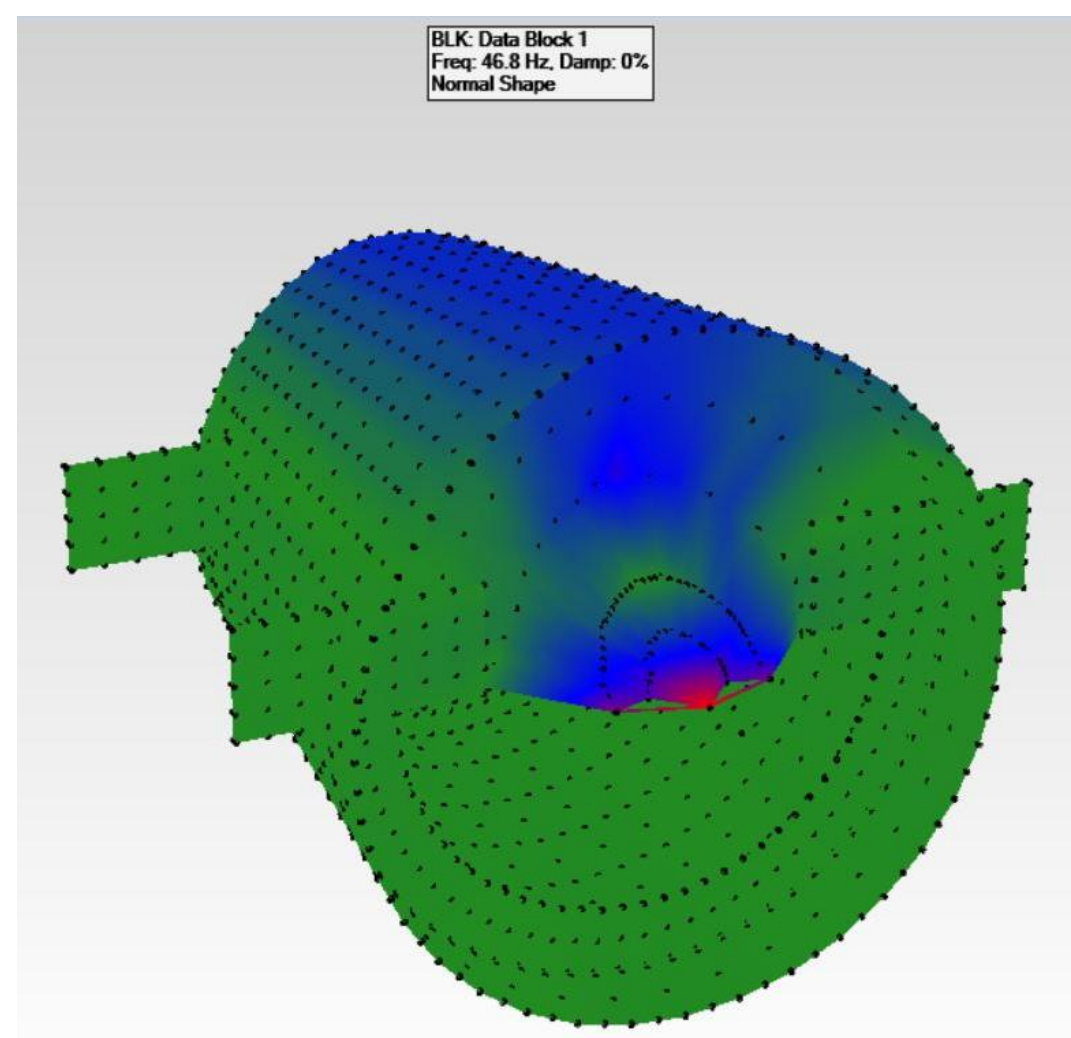

Figure B. 4: FESF Mode 4 @ $46.8 \mathrm{~Hz}$ 


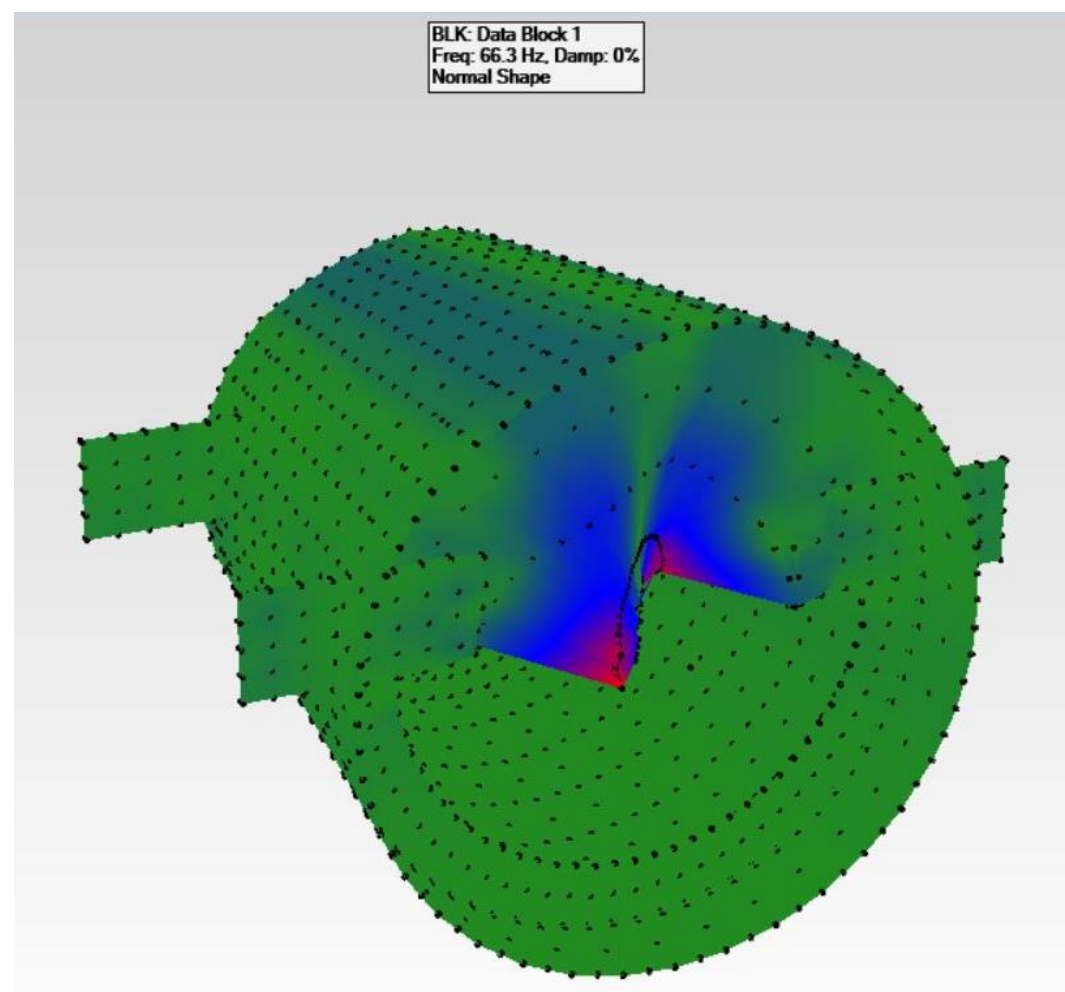

Figure B. 5: FESF Mode 5 @ $66.3 \mathrm{~Hz}$

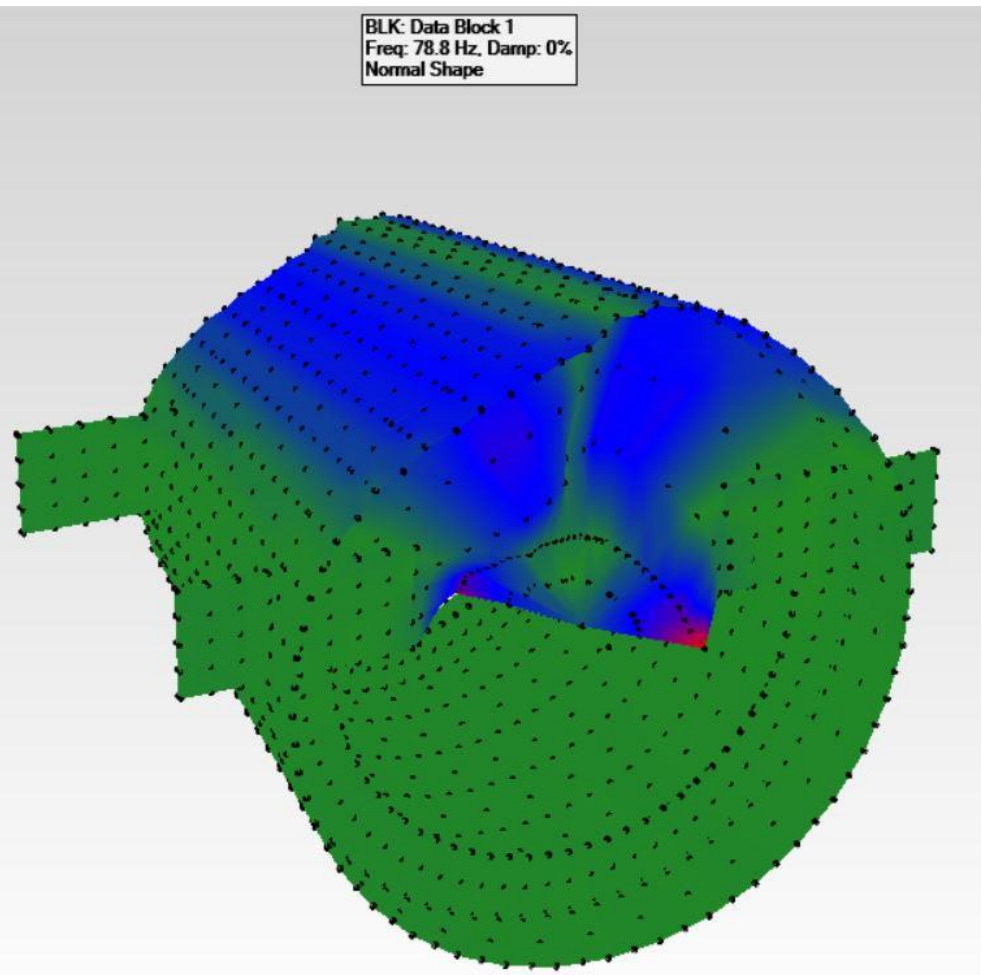

Figure B. 6: FESF Mode 6 @ $78.8 \mathrm{~Hz}$ 


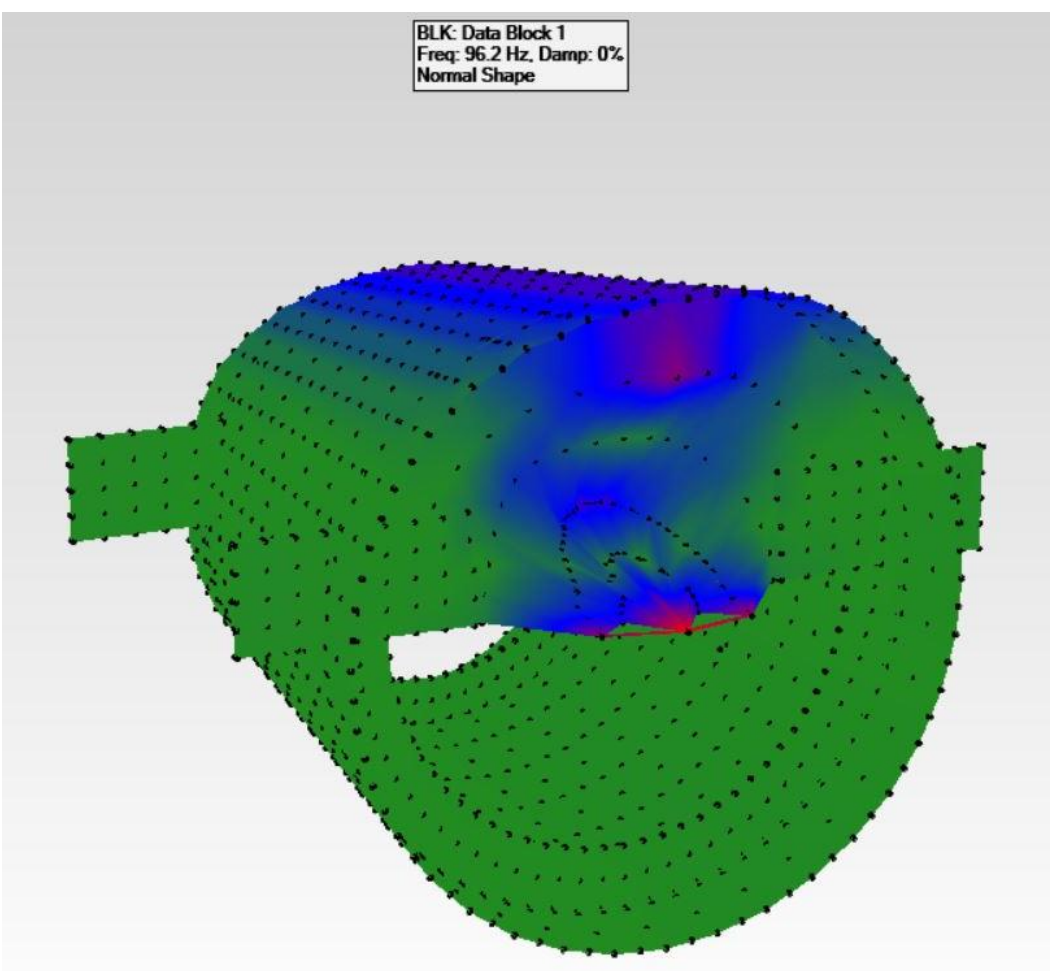

Figure B. 7: FESF Mode 7 @ 96.2 Hz

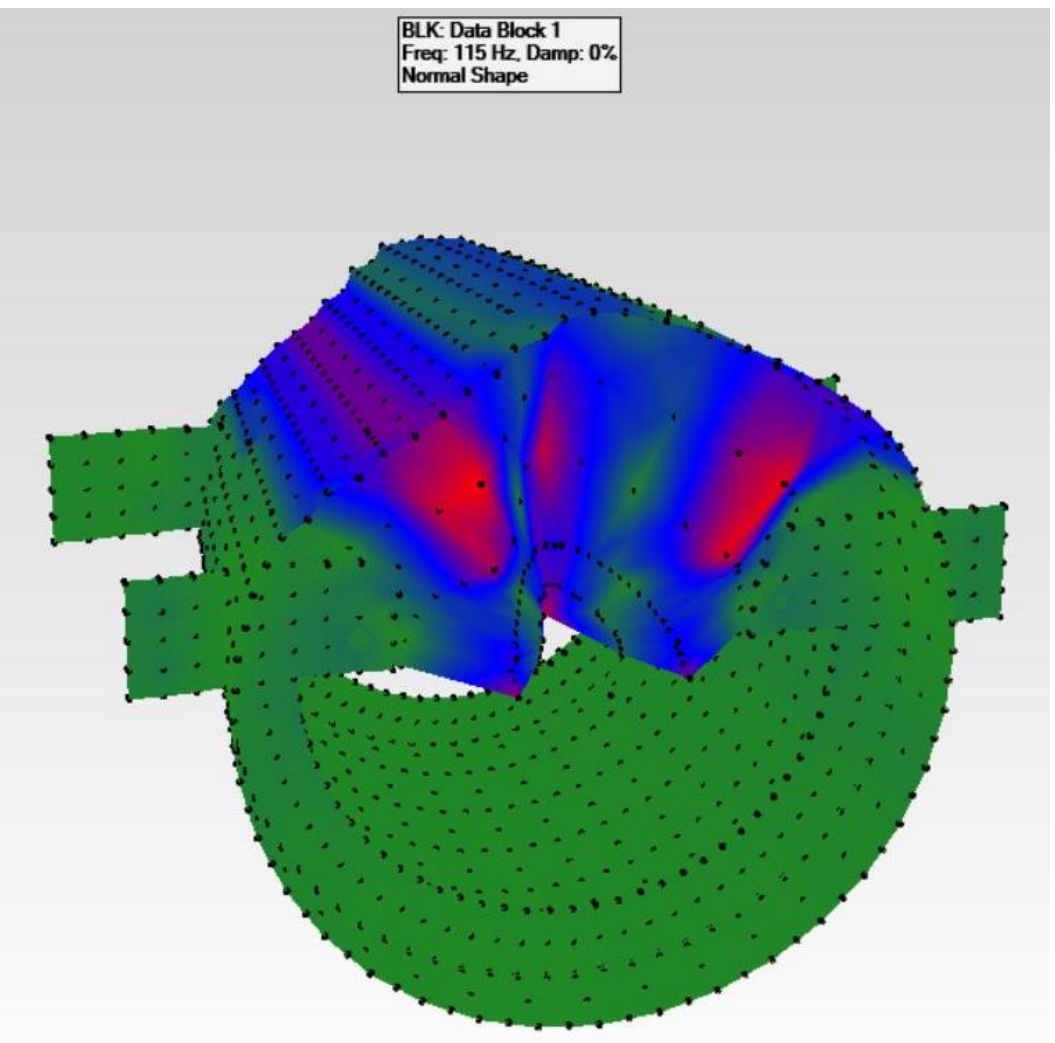

Figure B. 8: FESF Mode 8 @ 115 Hz 


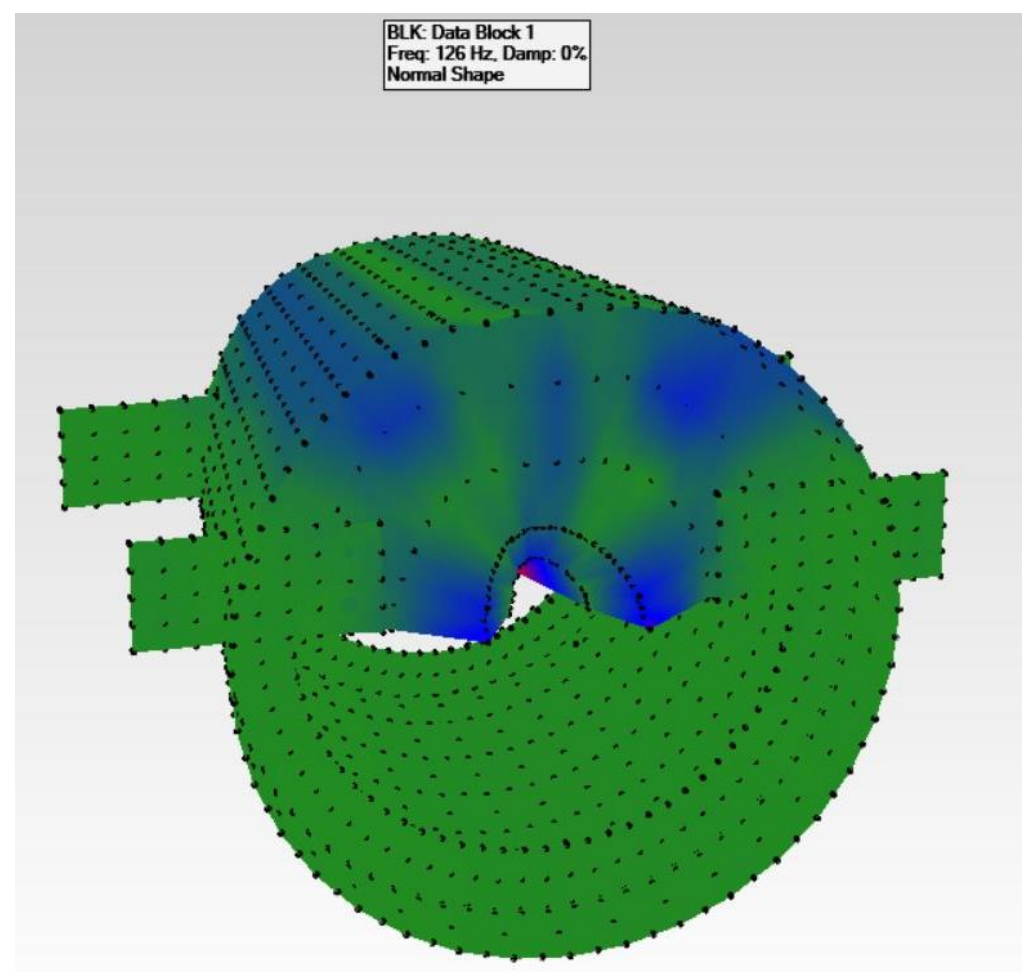

Figure B. 9: FESF Mode 9 @ $126 \mathrm{~Hz}$

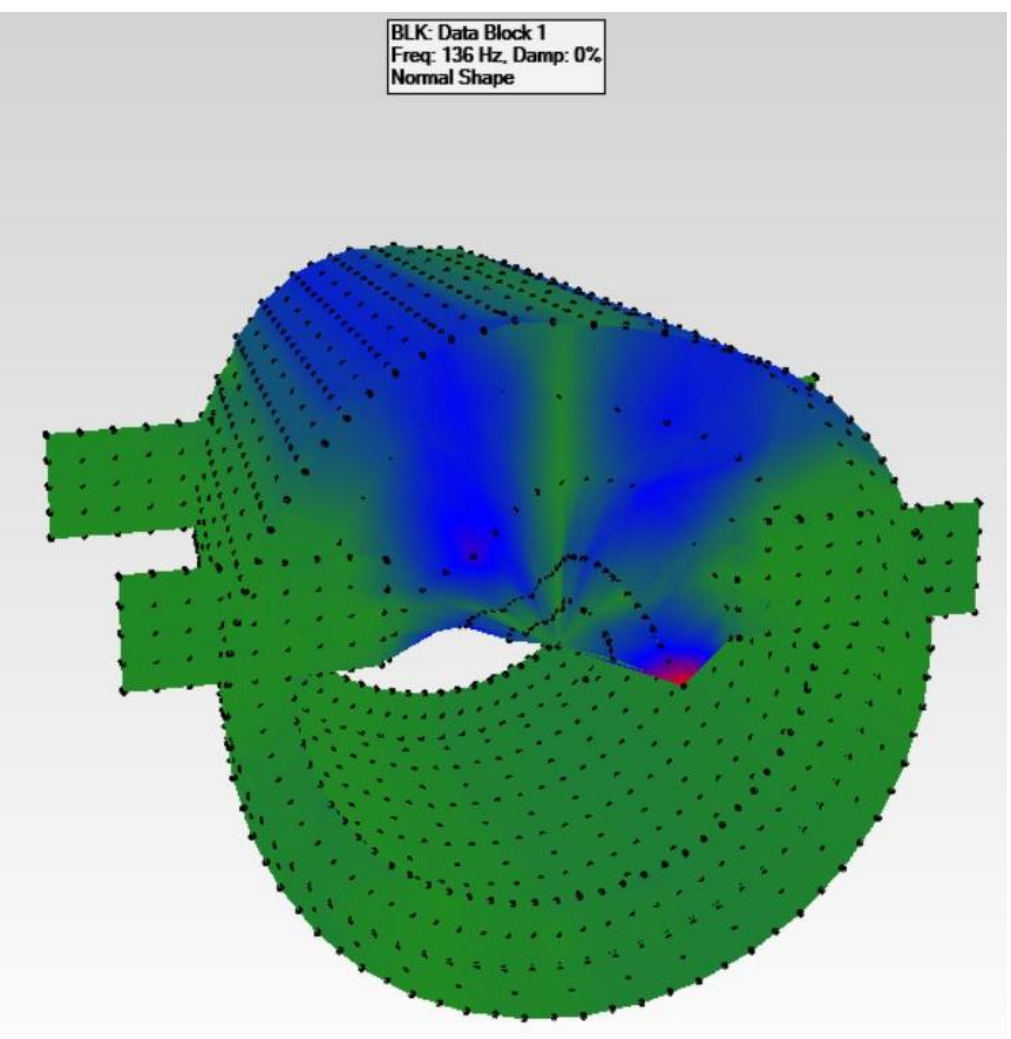

Figure B. 10: FESF Mode $10 @ 136 \mathrm{~Hz}$ 


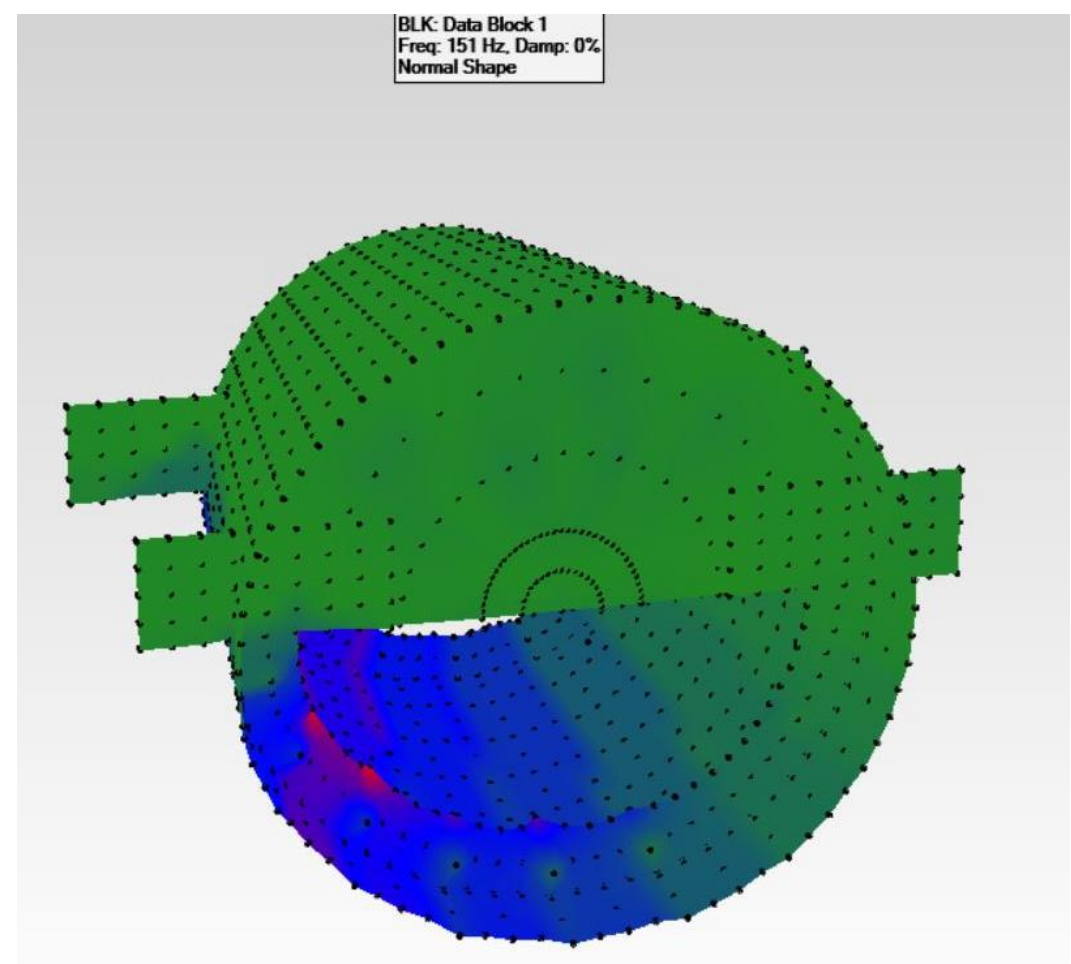

Figure B. 11: FESF Mode $11 @ 151 \mathrm{~Hz}$

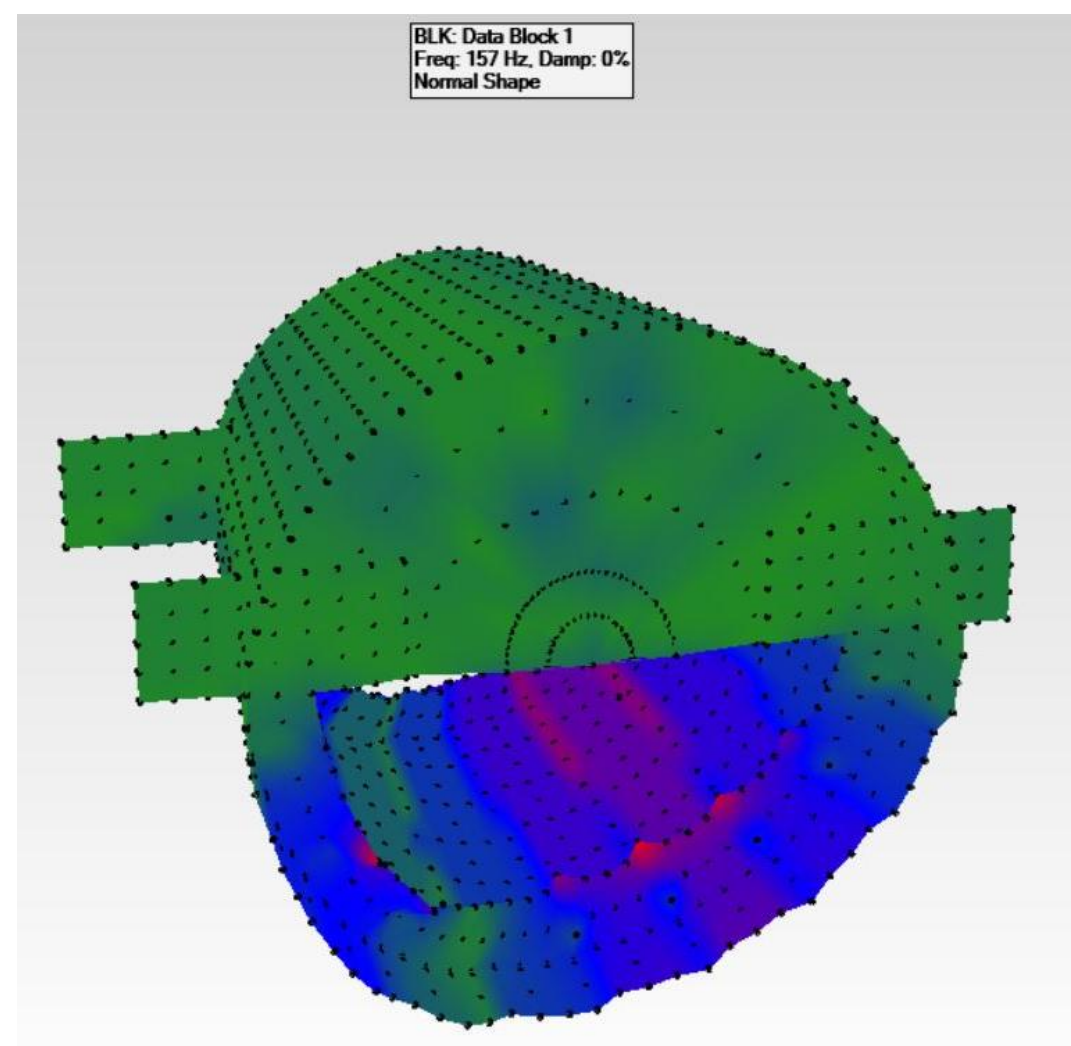

Figure B. 12: FESF Mode 12 @ 157 Hz 


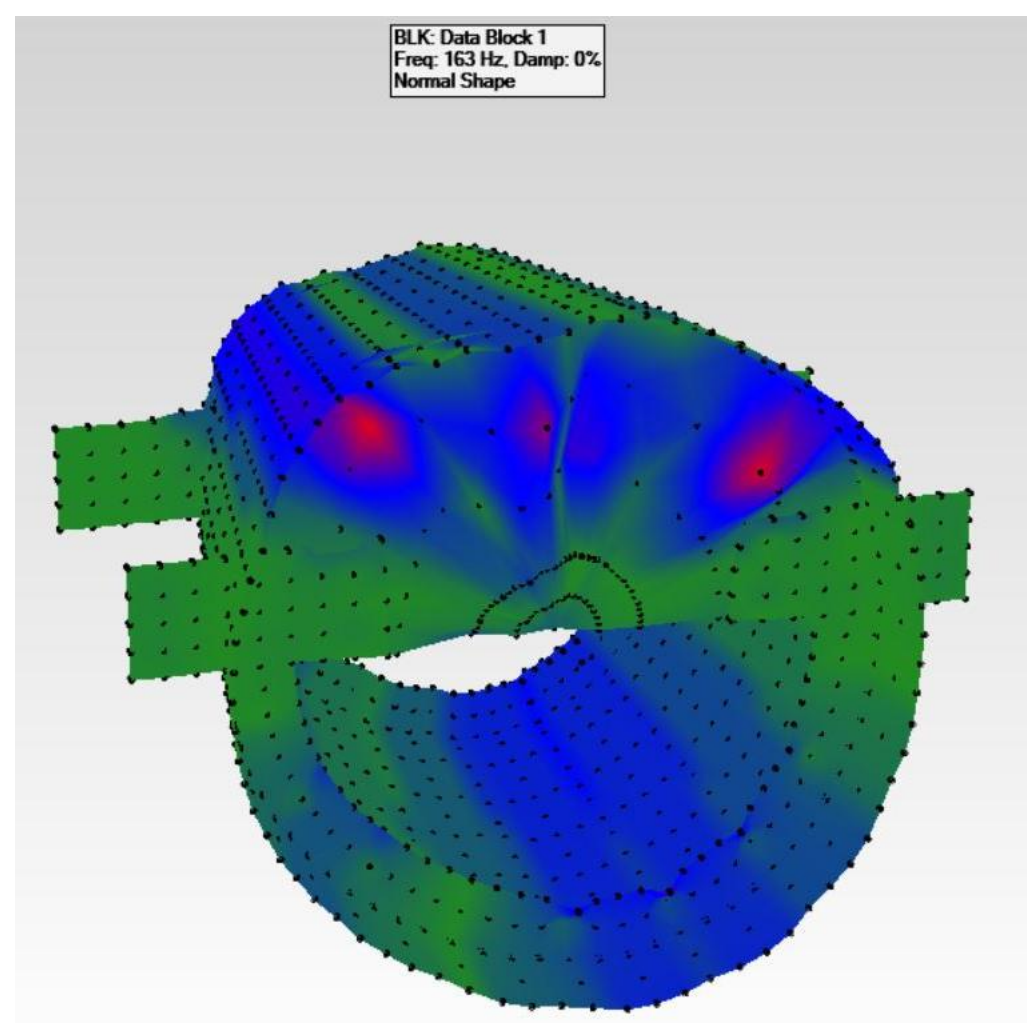

Figure B. 13: FESF Mode 13 @ 163 Hz

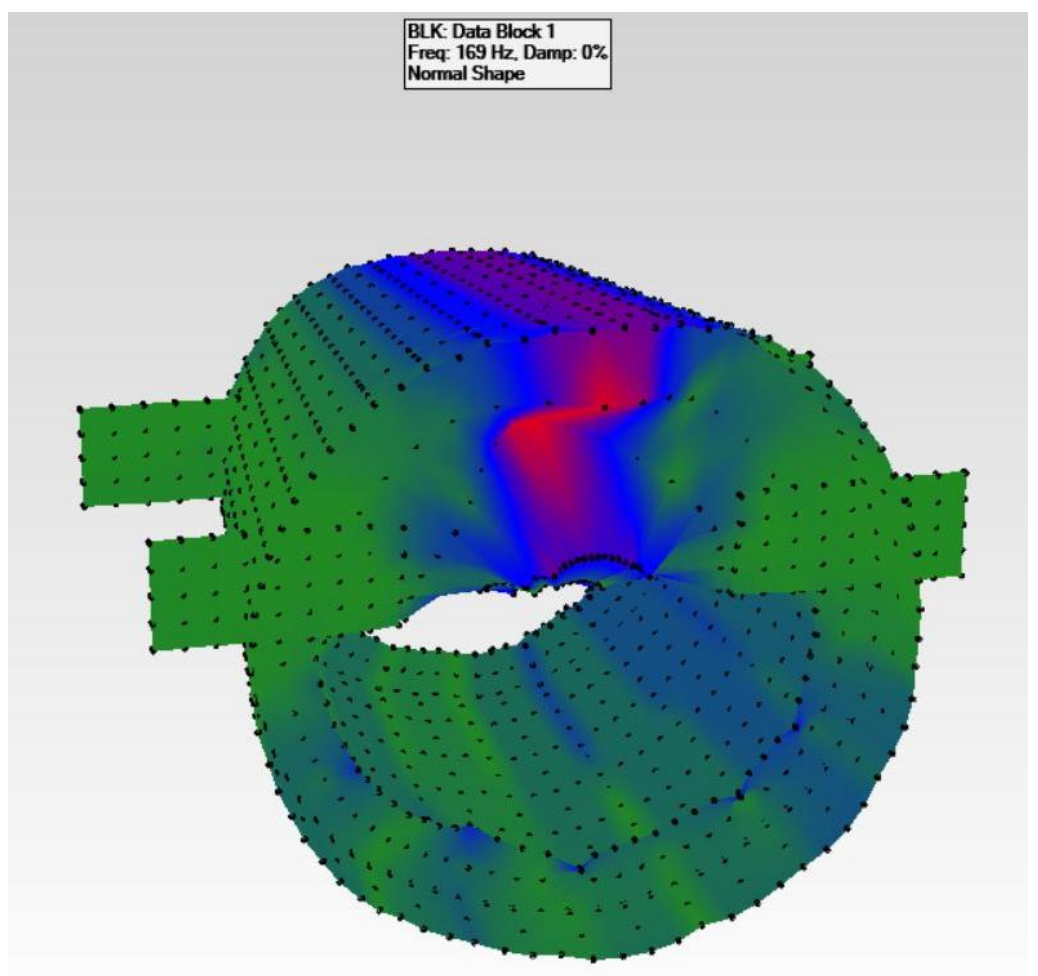

Figure B. 14: FESF Mode 14 @ 169 Hz 


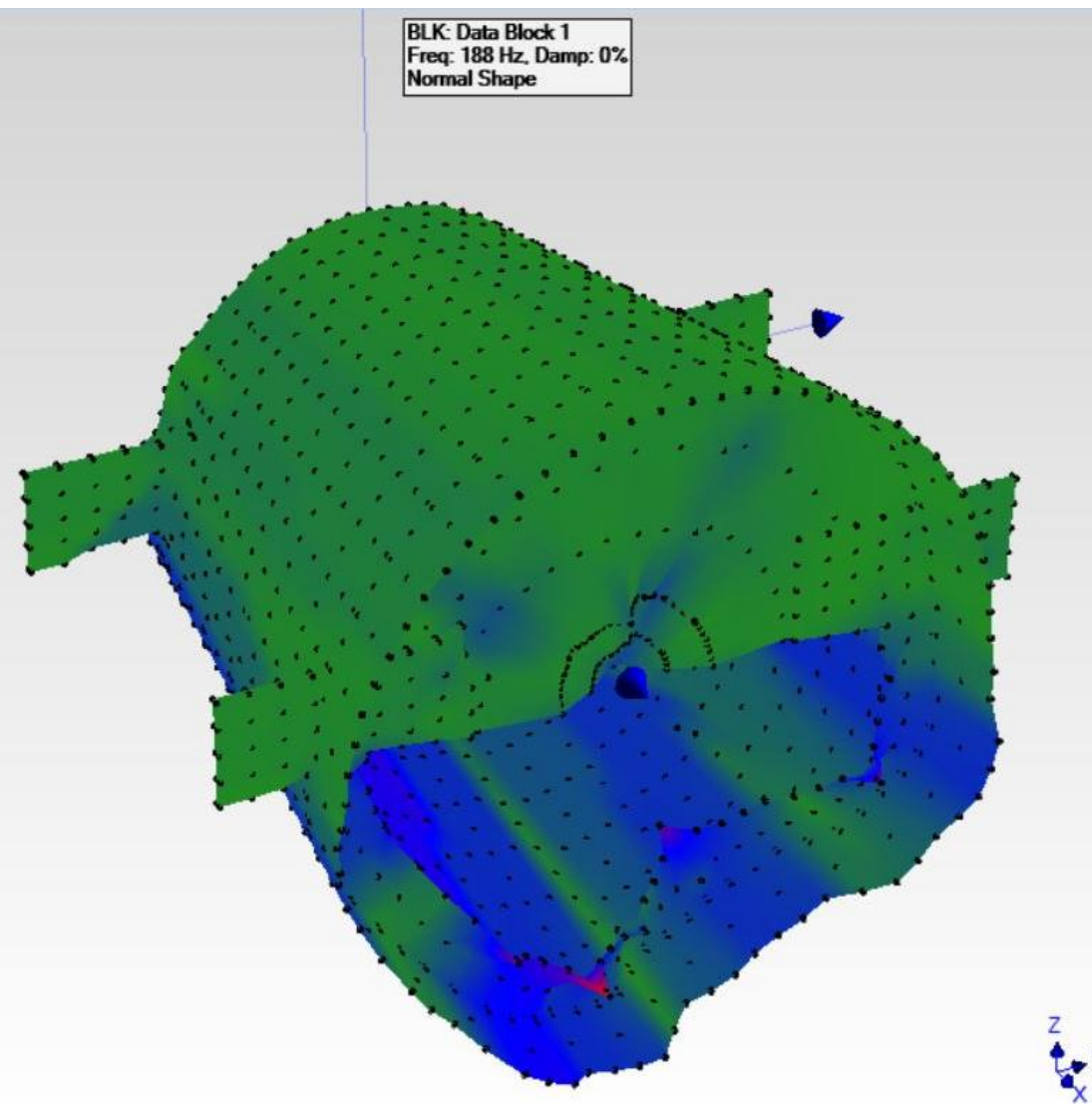

Figure B. 15: FESF Mode 15 @ $188 \mathrm{~Hz}$ 


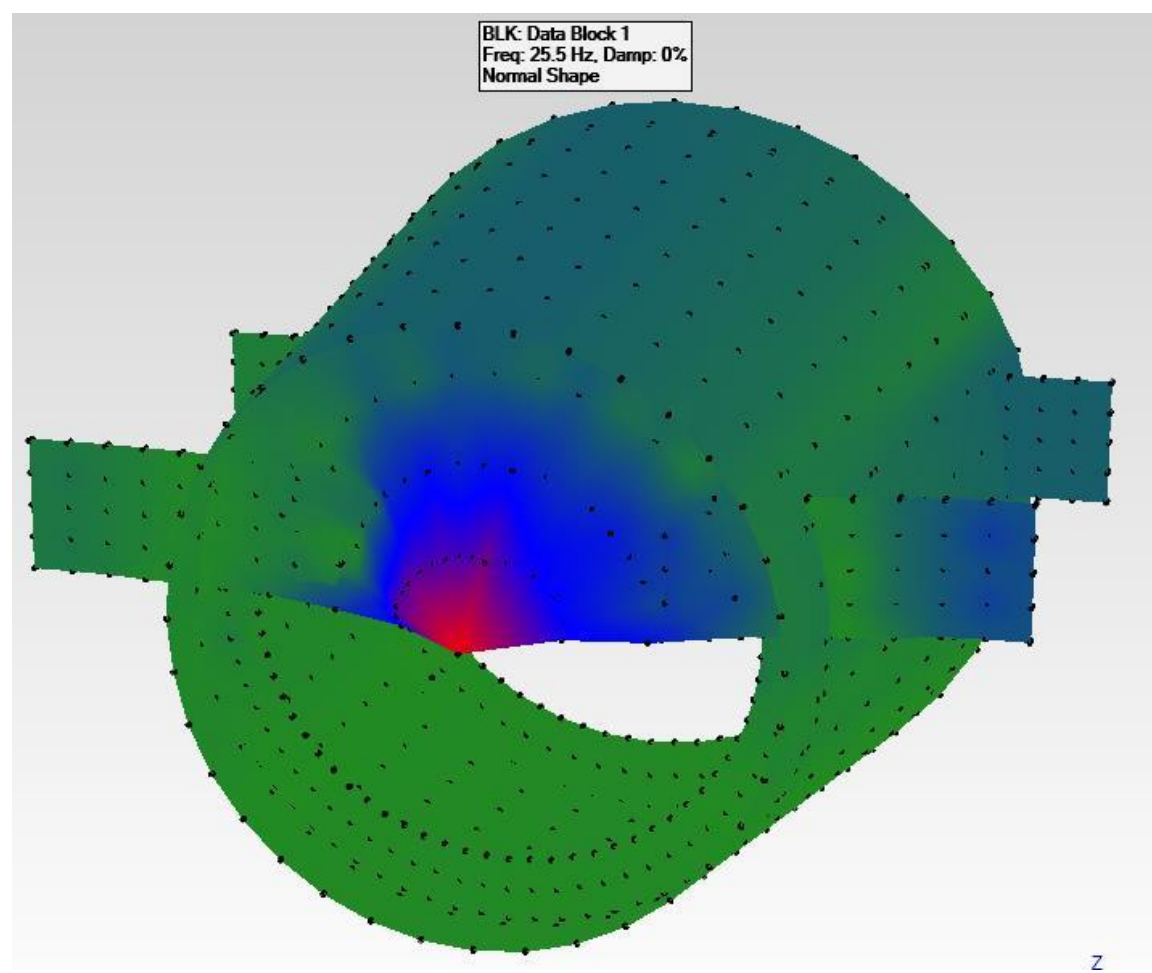

Figure B. 16: RESF Mode 1 @ 25.5 Hz

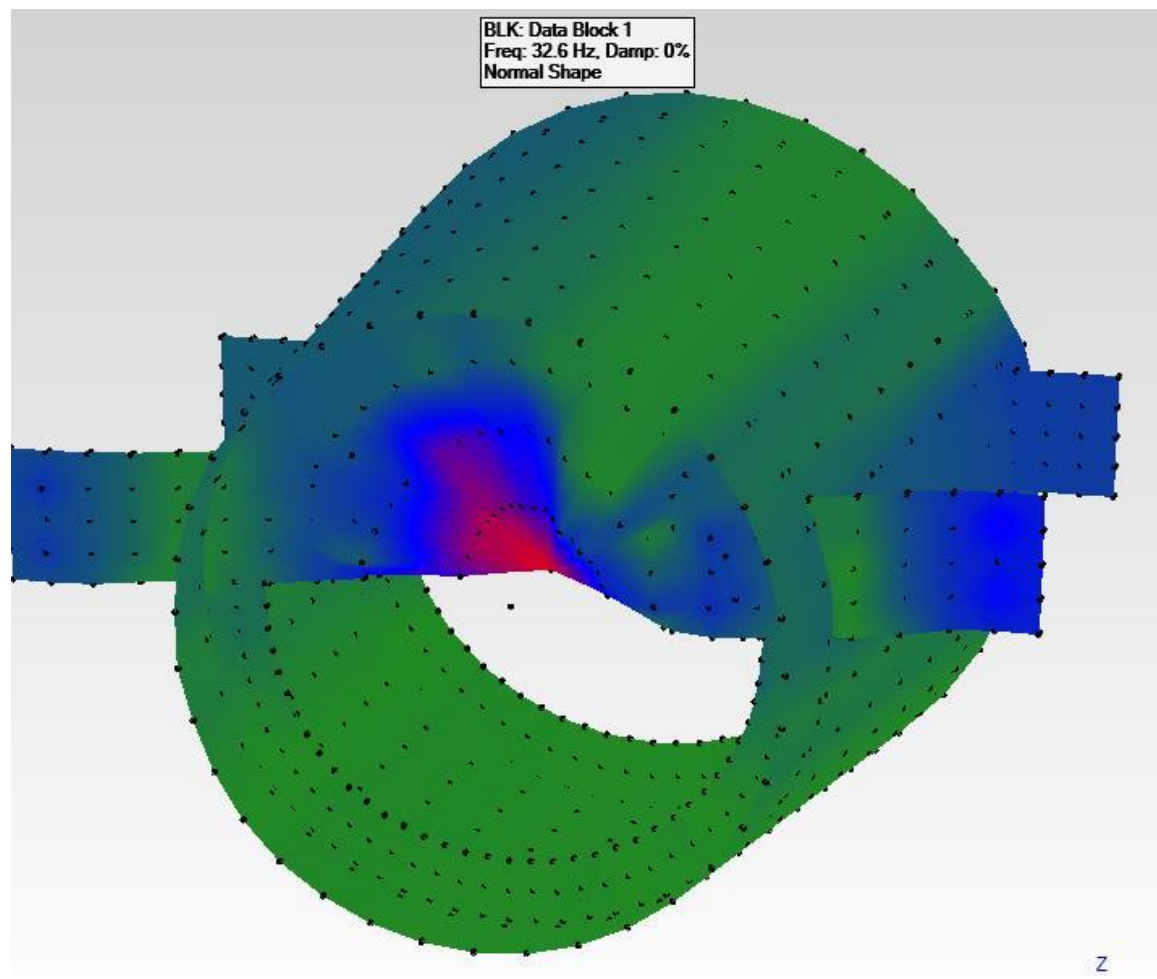

Figure B. 17: RESF Mode 2 @ $32.6 \mathrm{~Hz}$ 


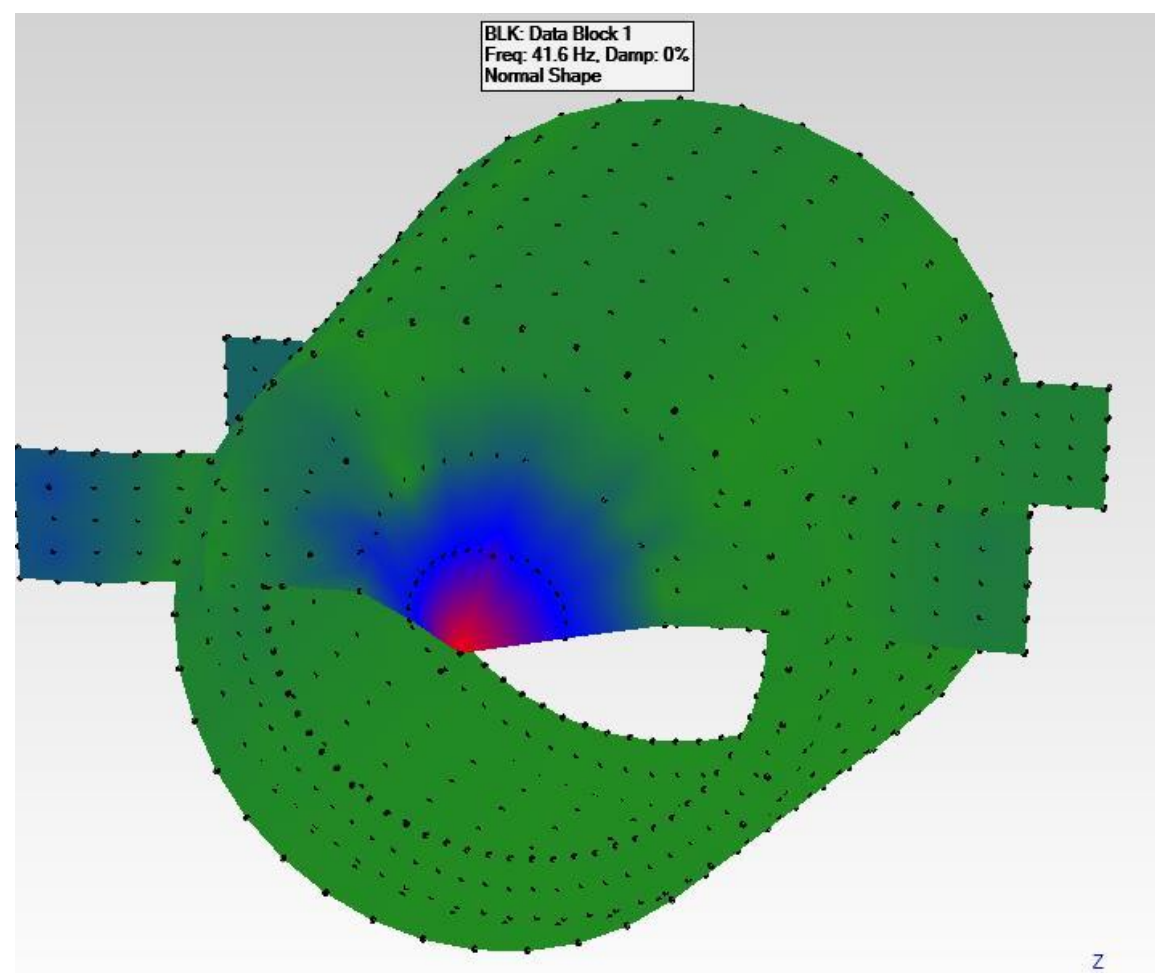

Figure B. 18: RESF Mode 3 @ 41.6 Hz

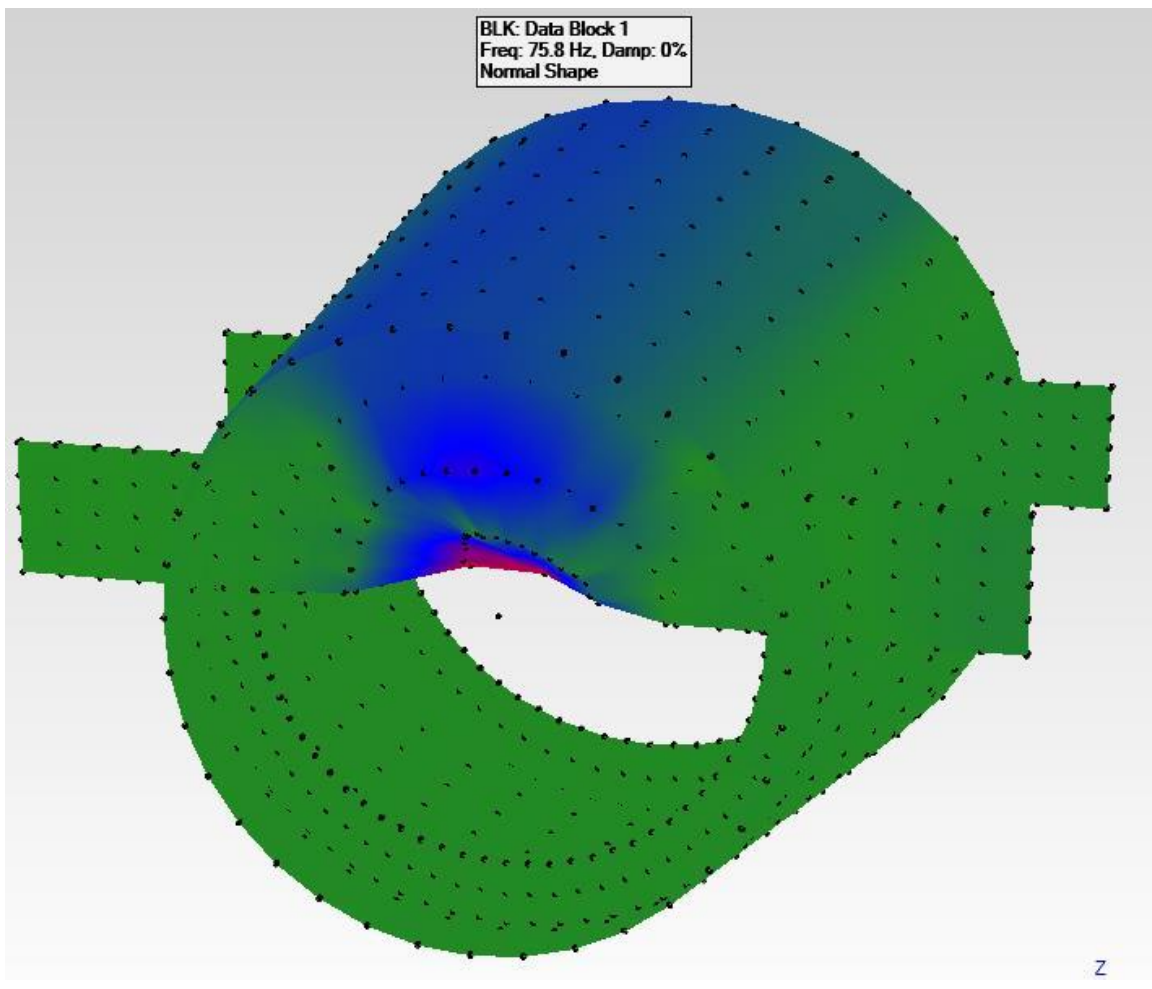

Figure B. 19: RESF Mode 4 @ $75.8 \mathrm{~Hz}$ 


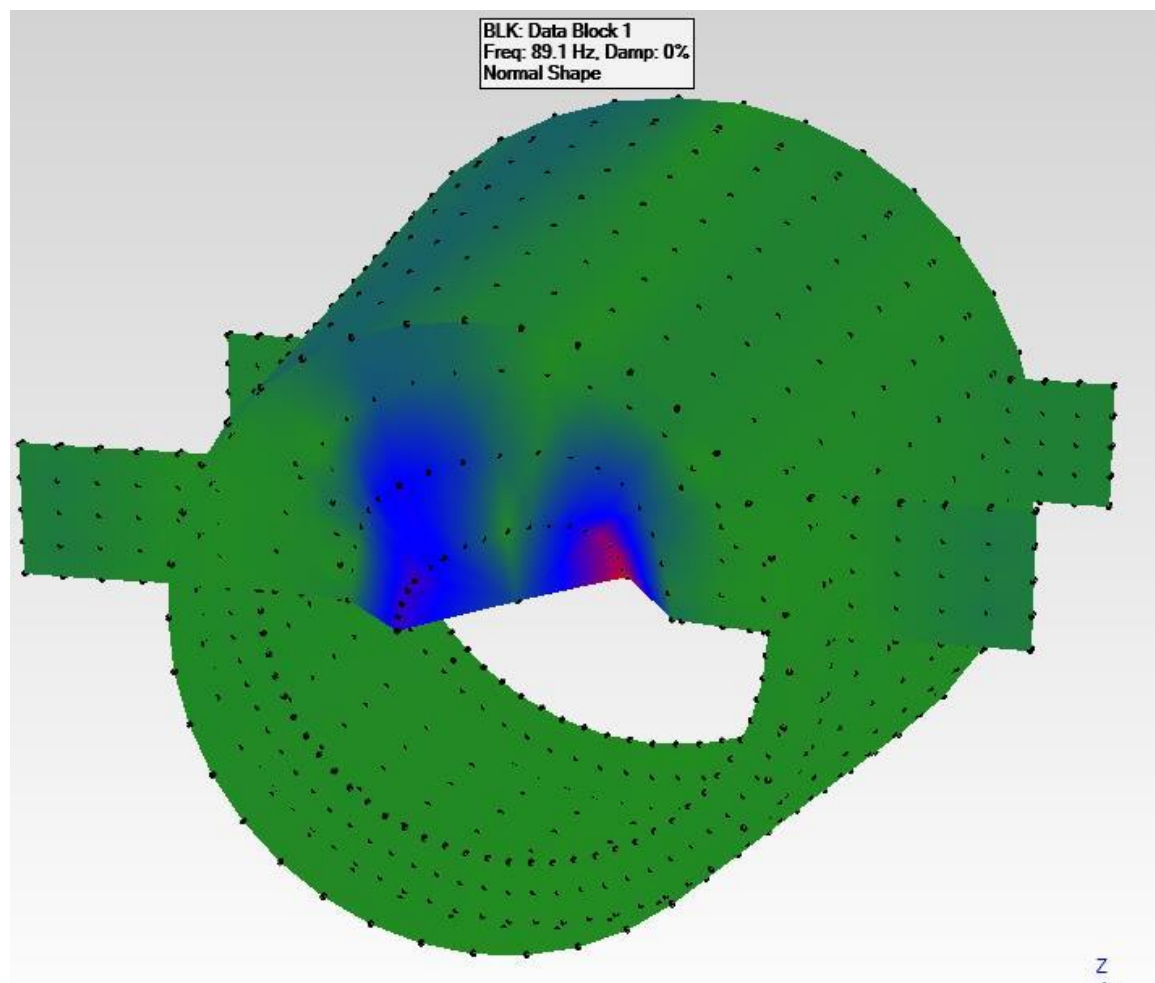

Figure B. 20: RESF Mode 5 @ 89.1 Hz

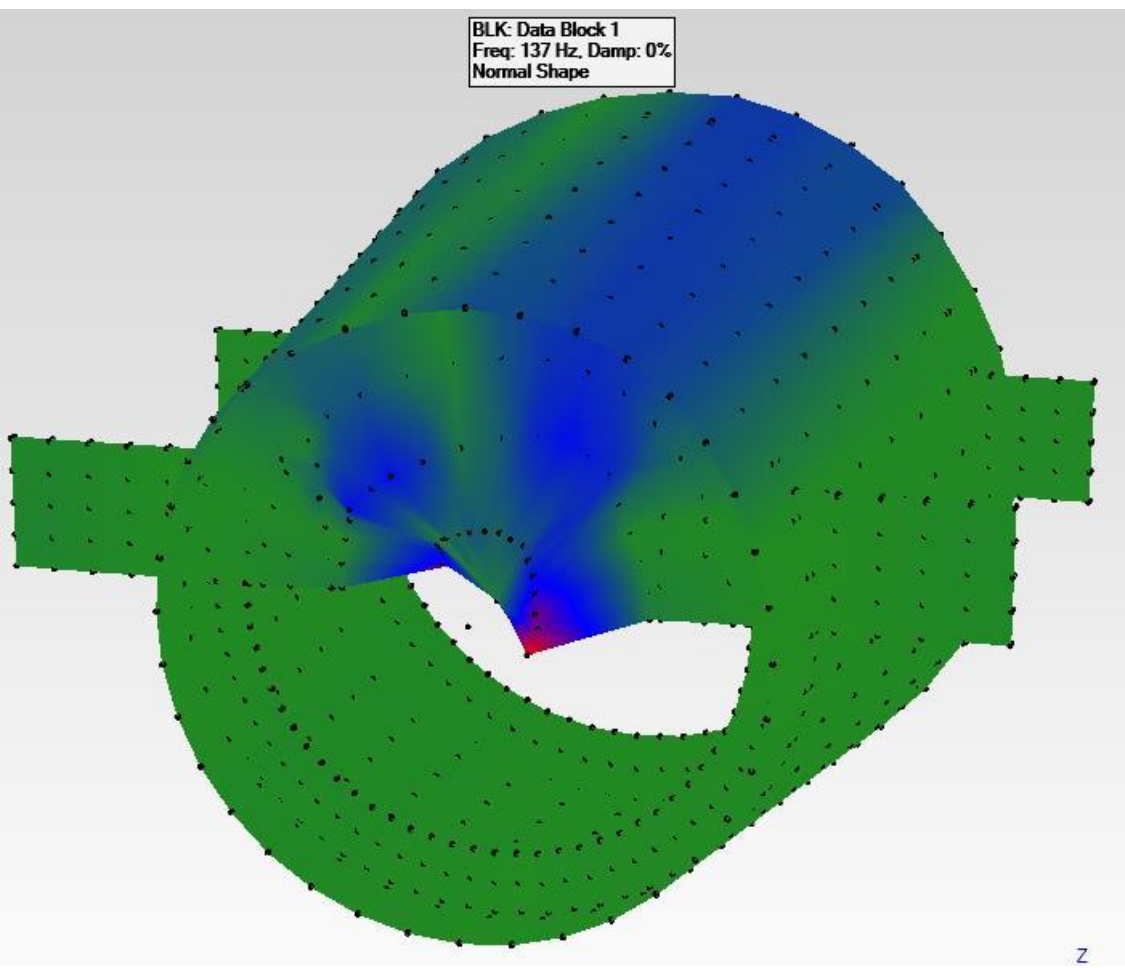

Figure B. 21: RESF Mode 6 @ $137 \mathrm{~Hz}$ 


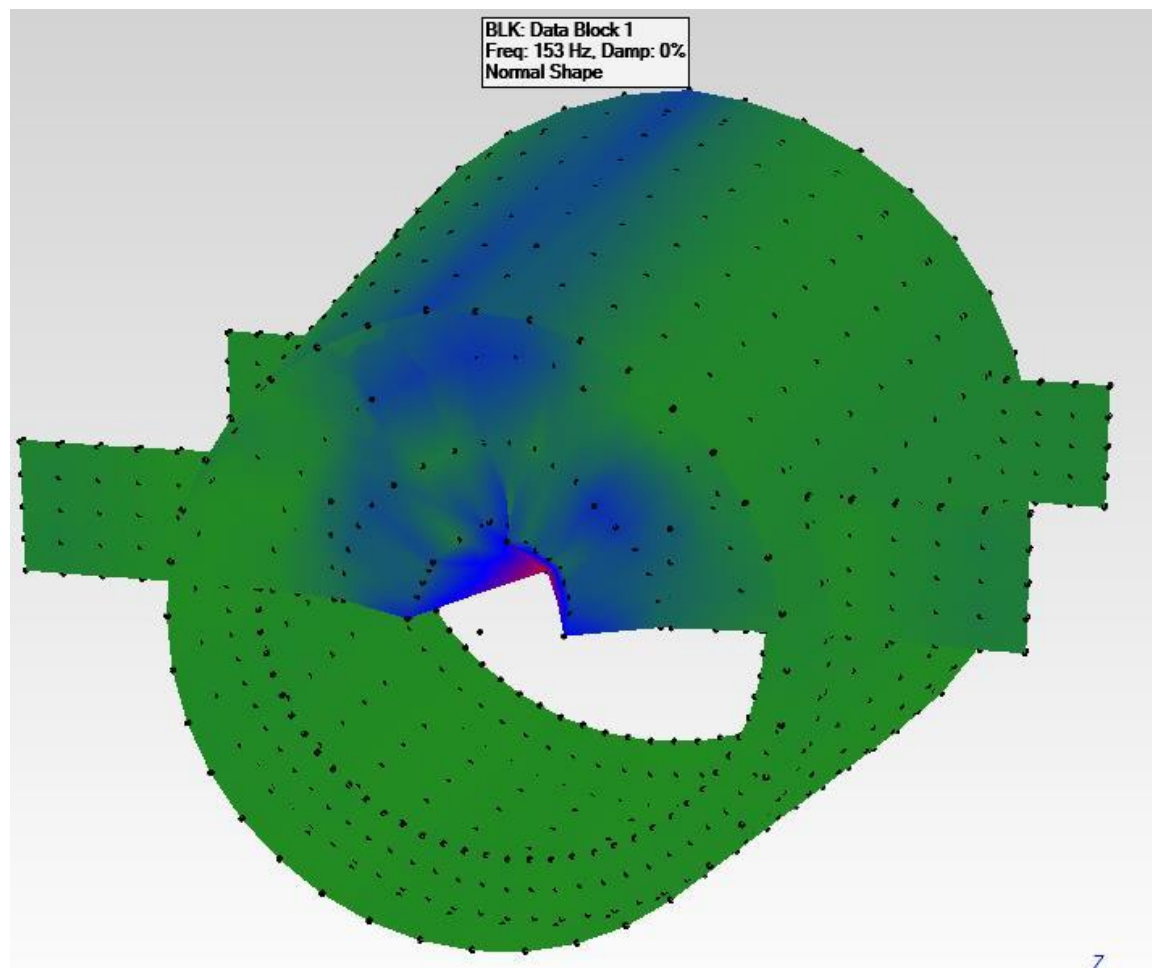

Figure B. 22: RESF Mode 7 @ $153 \mathrm{~Hz}$

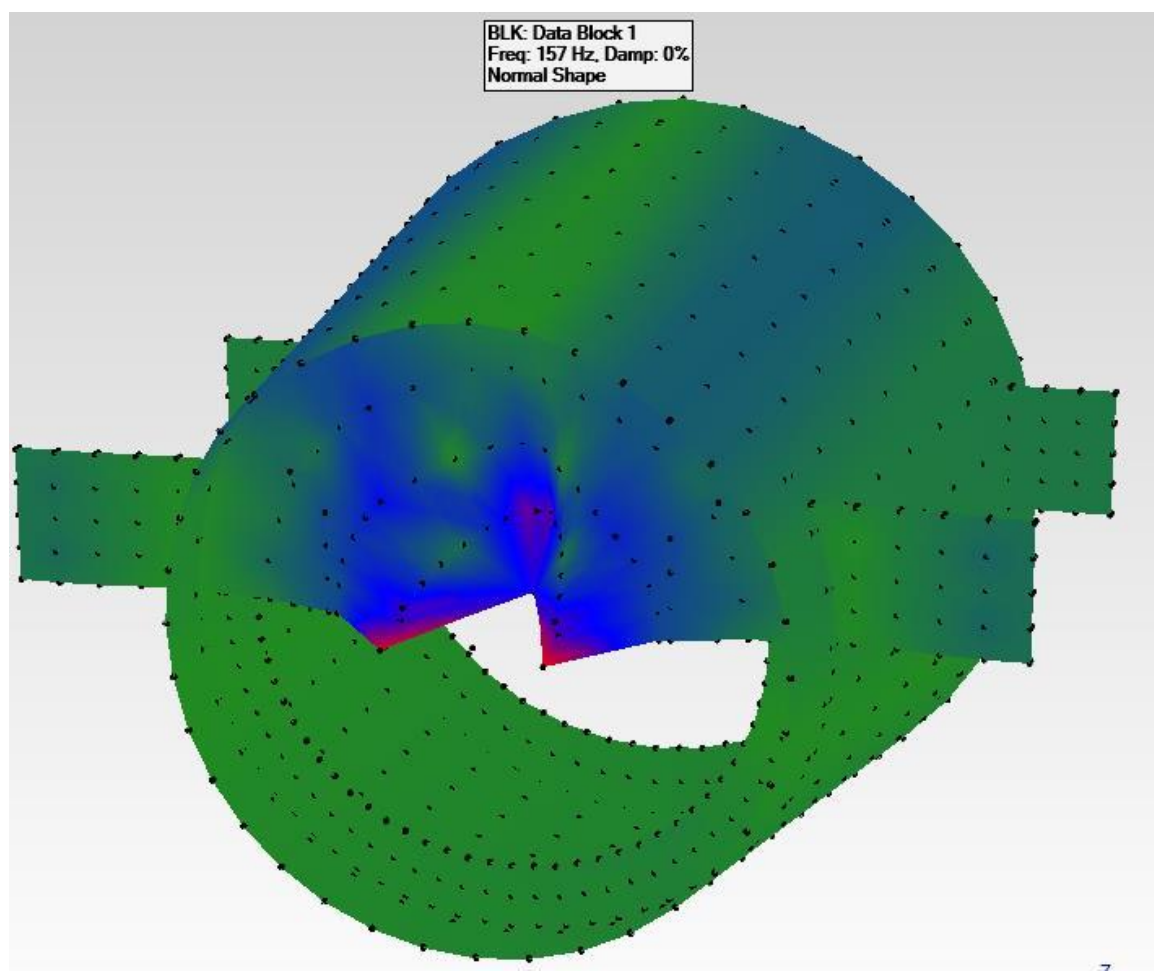

Figure B. 23: RESF Mode 8 @ $157 \mathrm{~Hz}$ 


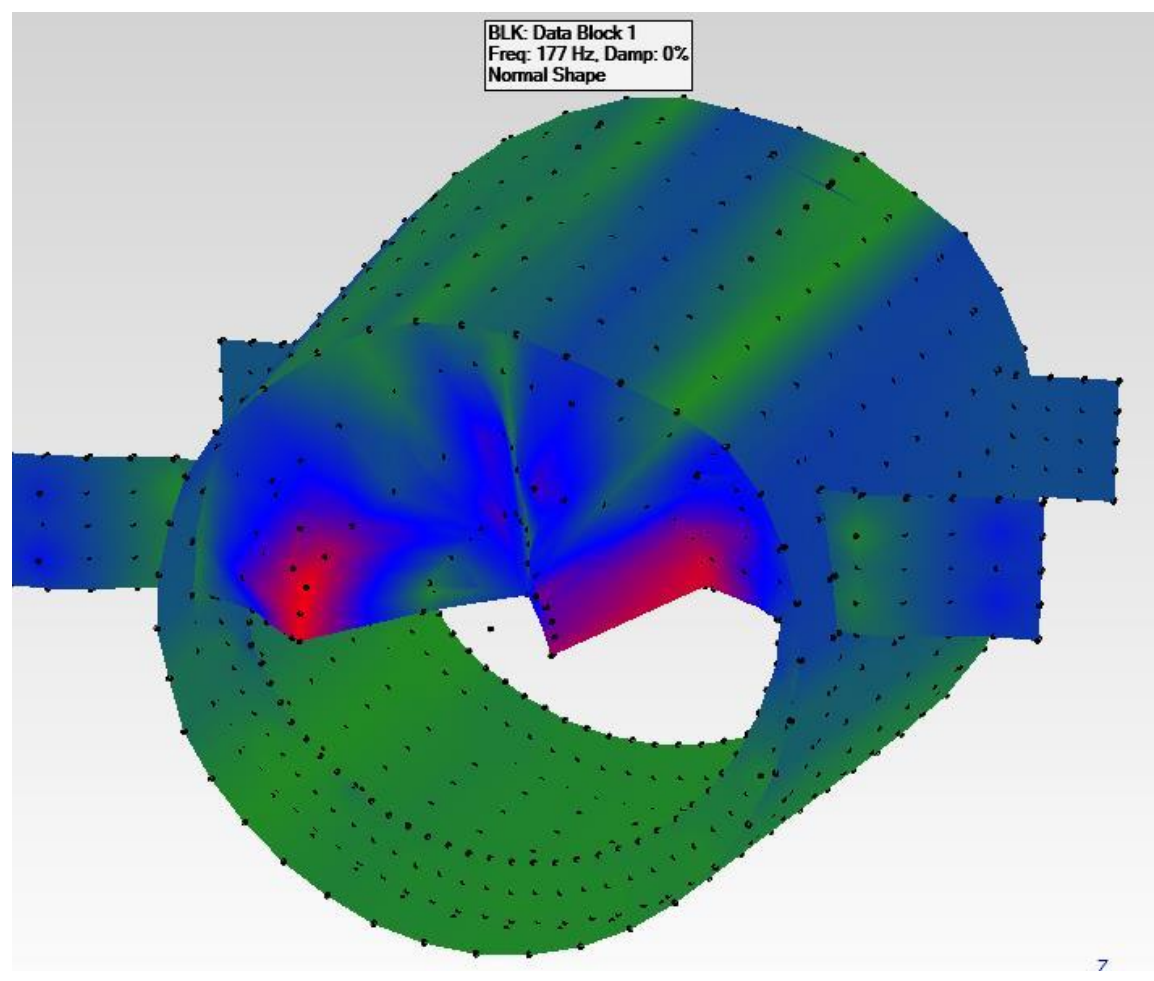

Figure B. 24: RESF Mode 9 @ 177 Hz

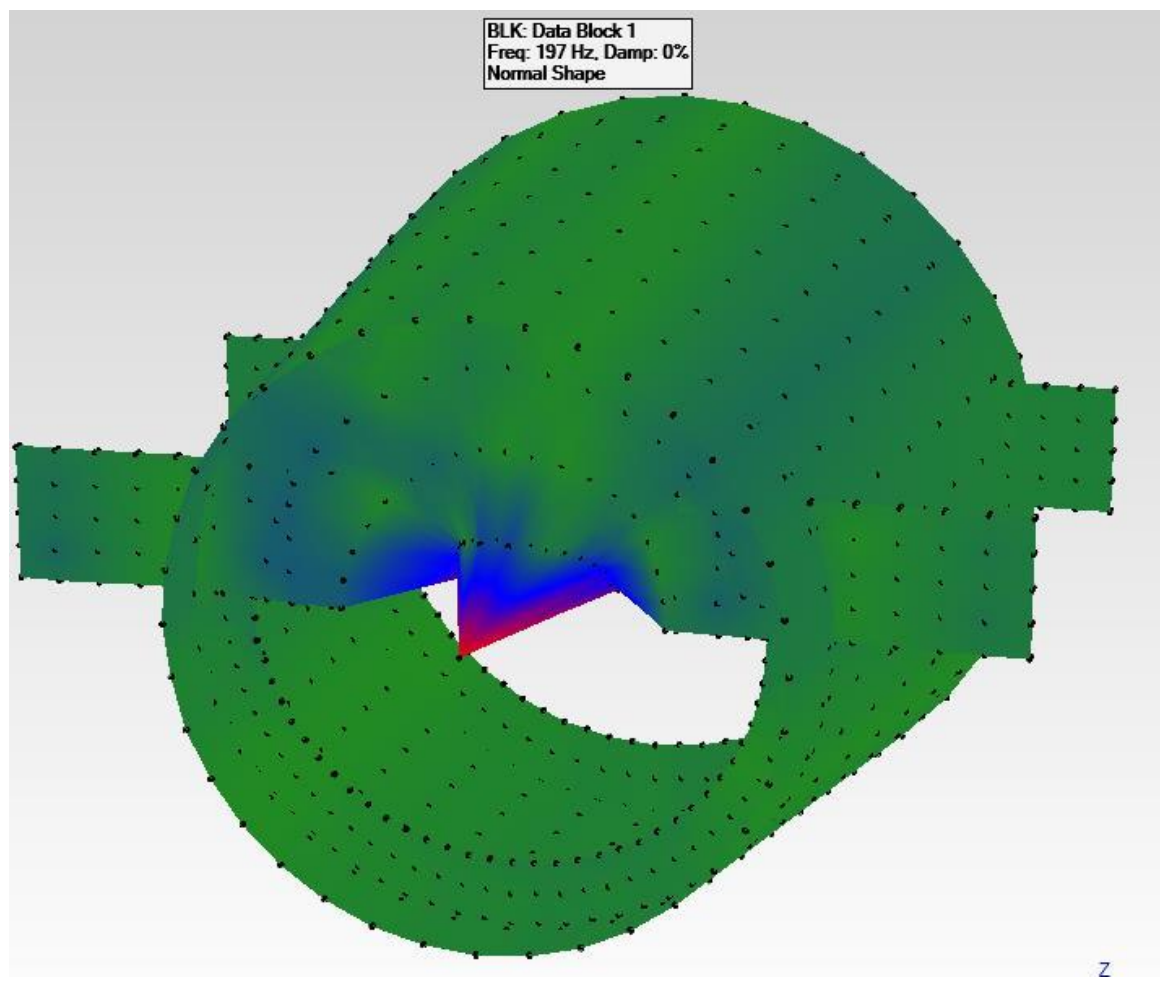

Figure B. 25: RESF Mode 10 @ $197 \mathrm{~Hz}$ 


\section{References}

Agilent Technologies. (1997). The Fundamentals of Modal Testing. Agilent Technologies.

Avitable, P. (2018). Modal Testing A Practitioners Guide. Hoboken: John Wiley and Sons.

Brown, D. L., \& Allemang, R. J. (2007). The Modern Era of Experimental Modal Analysis. Cincinati: University of Cincinati.

Bruel \& Kajer. (2008). Photon+ Dynamic Signal Analyser. Bruel and Kejer.

Chamberlain, D. A. (2018). Experimental Modal Analysis of a Half Scale Model Rear Twin-Engine Mounted Aircraft Fuselage Section. Kingston: Queen's University.

Cusano, A., Capoluongo, P., Campopiano, S., Cutolo, A., Giordano, M., Felli, F., . . Caponero, M. (2006). Experimental Modal Analysis of an Aircraft Model Wing by Embedded Fiber Bragg Grating Sensors. IEEE Sensors Journal Bolume 6, 67-77.

Dempsey, T. K., Leatherwood, J. D., \& Clevenson, S. A. (1978). Developement of Noise and Vibration Ride Comfort Criteria. Hampton, Virginia: National Aeronautics and Space Administration.

Ewins, D. (2000). Modal Testing Theory, Practice and Application. Philedelphia: Research Studies Press Ltd.

Fleming, G. A., \& Buerhle, R. D. (n.d.). Modal Analysis of an Aircraft Fuselage Panel using Experimental and Finite-Element Techniques. Hampton, Virginia: National Aeronautics and Space Administration.

Goge, D., Boswald, M., Fullekrug, U., \& Lubrina, P. (2007). Ground Vibration Testing of Large Aircraft-State-of-the-Art and Future Perspectives. IMAC . 
Gordon, R. W., Wolfe, H. F., \& Talmadge, R. D. (1977). Modal Investigation of Lightweight Aircraft Structures Using Digital Techniques. Dayton, Ohio: Air Force Flight Dynamics Laboratory .

International Organization for Standardization. (1994). ISO 7626 Vibration and ShockExperimental Determination of Mechanical Mobility. Geneva: International Orginization for Standardization.

Kehoe, M. W. (1995). A Historical Overview of Flight Flutter Testing. National Aeronautics and Space Administration.

Lam, C., \& Mechefske, C. (2020). COMPUTATIONAL MODAL ANALYSIS OF HALF SCALE GENERIC BUSINESS JET SUBSTRUCTURES. International Design Engineering Technical Conferences. St. Louis: American Society of Mechanical Engineers.

LDS-Dactron. (2004). RT Pro Dynamic Signal Analysis User Guide. Fremont, CA: LDS Test and Measuremetn Ltd.

Mixson, J. S., \& Kearny, B. C. (1978). Investigation of Interio Nois in a Twin-Engine Light Aircraft. New York, N.Y: National Aeronautics and Space Administration; Columbia University.

National Instruments. (2017, December 14). National Instruments. Retrieved from Measuring Vibration with Accelerometers: http://www.ni.com/white-paper/3807/en/\#toc4

National Intruments. (2019). Specifications PXI-1031/PXI-1031DC. National Instruments.

PCB Piezotronics. (2020). Model 288D01. (PCB Piezotronics) Retrieved February 6, 2020, from https://www.pcb.com/products?model=288D01

PCB Piezotronics. (2020). Model 352C22. (PCB Piezotronics) Retrieved January 14, 2020, from https://www.pcb.com/products? model=352C22 
Peres, M. A., Bono, R. W., \& Avitable, P. (2012). Effects of shaker, stinger and transducer mounting on measured frequency response functions. International Conference on Noise and Vibration Engineering, (pp. 1475-1490). Leuven.

Peres, M., Bono, R., \& Brown, D. (2010). Practical Aspects of Shaker Measurements for Modal Testing. International Conference on Noise and Vobration Engineering. Leuven.

Vibrant Technology. (2015). ME'ScopeVES Reference Manual Basic Operations. Centennial, Co: ibrant Technology.

Warwick, B. T., Kim, I. Y., \& Mechefske, C. K. (2019). Substructuring verification of a rear fuselage mounted twin-engine aircraft. Aerospace Science and Technology, 93.

Warwick, B. T., Mechefske, C. K., \& Kim, I. Y. (2018). Effect of Stiffener Configuration on Bulkhead Modal Parameters. International Design Engineering Technical Conferences. Quebec City, Canada: The American Society of Mechanical Engineers.

Warwick, B. T., Mechefske, C. K., \& Kim, I. Y. (2019). Topology optimization of a pre-stiffened aircraft bulkhead. Structural and Multidisciplinary Optimization, 60(4), 1667-1685.

Wilby, J. F. (1995). Aircraft Interior Noise. Calabasas, CA: Academic Press Limited.

Wyen, T. A., Schoettelkotte, J. J., Perez, R. A., \& Eason, T. (2017). Experimental Modal Analysis of an Aircraft Fuselage Panel. Shock and Vibration, Aircraft/Aerospace, Energy Harvesting, Acoustics and Optics, Volume 9 (p. 12). Dayton Ohio: Society For Experimental Mechanics. 